Supplementary Information for

\title{
A Highly Strained Al-Al $\sigma$-Bond in Dianionic Aluminum Analog of Oxirane for Molecule Activation
}

\author{
Kota Koshino, ${ }^{1}$ Rei Kinjo ${ }^{1, *}$ \\ 1. Division of Chemistry and Biological Chemistry, \\ School of Physical and Mathematical Sciences, \\ Nanyang Technological University, 637371, Singapore
}

Correspondence to: rkinjo@ntu.edu.sg 


\section{Contents}

1. Synthesis of compounds $\mathbf{2}-\mathbf{8}$ and their spectral data

2. Crystal structural parameters for $\mathbf{2}-\mathbf{8}$

3. Theoretical calculations

4. References 


\section{Synthesis of compounds $2-8$ and their spectral data}

\section{General considerations}

All reactions except for synthesis of $\mathbf{4}$ were performed under an atmosphere of argon in a glove box; solvents were dried over $\mathrm{Na}$ metal, $\mathrm{K}$ metal. Reagents were of analytical grade, obtained from commercial suppliers and used without further purification. ${ }^{1} \mathrm{H},{ }^{13} \mathrm{C}$ NMR spectra were obtained with Bruker $400 \mathrm{MHz}$ BBFO spectrometers at $298 \mathrm{~K}$ unless otherwise stated. ${ }^{27} \mathrm{~A} \mid\left\{{ }^{1} \mathrm{H}\right\}$ and ${ }^{29} \mathrm{Si}\left\{{ }^{1} \mathrm{H}\right\}$ were obtained with JEOL ECA400 and ECA400SL unless otherwise stated. NMR multiplicities are abbreviated as follows: $s=$ singlet, $d=$ doublet, $m=$ multiplet, $b r$ $=$ broad signal. Coupling constants $J$ are given in $\mathrm{Hz}$. Electrospray ionization (ESI) mass spectra were obtained at the Mass Spectrometry Laboratory at the Division of Chemistry and Biological Chemistry, Nanyang Technological University. Melting points were measured with OptiMelt (Stanford Research Systems). Fourier transform infrared (FT-IR) spectrum was recorded on a Bruker ALPHA-Transmittance FT-IR Spectrometer. UV-vis absorption spectra were measured with Cary 300 UV-vis spectrometer. Elemental analysis was carried out at the Elemental Analysis Laboratory at the Division of Chemistry and Biological Chemistry, Nanyang Technological University. Dialane 1 was synthesized according to the literature procedure. ${ }^{[S 1]}$

\section{Synthesis of 2.}

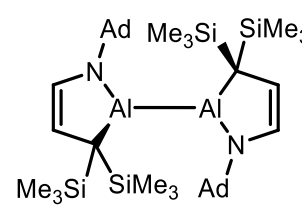

1

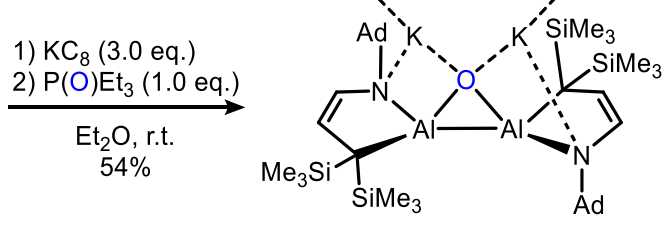

2

Et2O $(3.0 \mathrm{ml})$ was added to a mixture of dialane $(4) 1(200 \mathrm{mg}, 0.28 \mathrm{mmol})$ and $\mathrm{KC}_{8}(112 \mathrm{mg}$, $0.83 \mathrm{mmol}$ ) at room temperature in a vial. The mixture was stirred for 1 hour and the solution part was separated by filtration. The resulting solution was concentrated to $1.0 \mathrm{~mL}$, then treated with triethylphosphine oxide $(37.2 \mathrm{mg}, 0.28 \mathrm{mmol})$ and stirred for 15 minutes, affording a large amount of yellow crystals. The suspension was stored at $-5^{\circ} \mathrm{C}$ for $16 \mathrm{~h}$. During this time yellow crystals suitable for X-ray diffraction analysis were formed. The mother liquid was removed, and the yellow crystals were washed with $\mathrm{Et}_{2} \mathrm{O}(1.0 \mathrm{~mL} \times 3)$, and dried under vacuum to afford 2 as a yellow solid (226 mg, 54\%). 
M.p.: 159-160 ㅇ (dec.); ${ }^{1} \mathrm{H}$ NMR (THF-d8, $\left.400 \mathrm{MHz}, 298 \mathrm{~K}\right): \delta 6.59$ (d, J = 5.6 Hz, 2H, CH), 4.39 (d, J = 5.6 Hz, 2H, CH), 2.05 (br, 12H, Ad-CH2), 1.99 (br, 6H, Ad-CH), 1.75-1.67 (m, 6H,

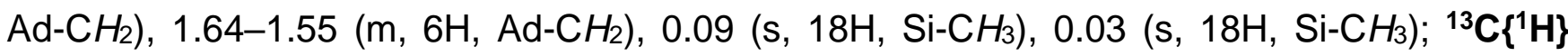
NMR (THF-d8, $100 \mathrm{MHz}, 298 \mathrm{~K}): \delta 137.8(\mathrm{CH}), 101.5(\mathrm{CH}), 53.7(\mathrm{Ad}-\mathrm{qC}), 47.1\left(\mathrm{Ad}-\mathrm{CH}_{2}\right), 37.8$ $\left(\mathrm{Ad}-\mathrm{CH}_{2}\right), 31.3(\mathrm{Ad}-\mathrm{CH}), 17.8\left(\mathrm{AlC}_{3} \mathrm{~N}-\mathrm{q} C\right), 3.74\left(\mathrm{Si}-\mathrm{CH}_{3}\right), 2.84\left(\mathrm{Si}-\mathrm{CH}_{3}\right) ;{ }^{27} \mathrm{Al}\left\{{ }^{1} \mathrm{H}\right\}$ NMR $\left(\mathrm{THF}-\mathrm{d}_{8}\right.$, $104 \mathrm{MHz}, 298 \mathrm{~K}$ ): $\delta 147.3(\mathrm{br}) ;{ }^{29} \mathrm{Si}\left\{{ }^{1} \mathrm{H}\right\}$ NMR (THF-d8, $80 \mathrm{MHz}, 298 \mathrm{~K}$ ) $\delta-4.61,-7.34$; FT-IR (solid, $\mathrm{cm}^{-1}$ ): 2954, 2890, 2835, 1558, 1441, 1365, 1301, 1228, 1206, 1176, 1143, 1094, 1067, 967, 948, 884, 852, 823, 800, 776, 752, 723, 694, 674, 657, 625; UV-vis (THF): $\lambda_{\max }=340$ nm; HRMS $\left(\mathrm{ESI}^{-}\right): \mathrm{m} / z$ calcd for $\mathrm{C}_{38} \mathrm{H}_{70} \mathrm{~N}_{2} \mathrm{OAl}{ }_{2} \mathrm{Si}_{4} \mathrm{~K}\left[M-K\left(O E t_{2}\right)_{2}\right]^{-}$775.3833, found 775.3835; Anal. Calc. for $\mathrm{C}_{46} \mathrm{H}_{90} \mathrm{Al}_{2} \mathrm{~K}_{2} \mathrm{~N}_{2} \mathrm{O}_{3} \mathrm{Si}_{4}$ : C 57.33\%, H 9.41\%, N 2.91\%, found: C 57.34\%, H 9.59\%, N $2.91 \%$.

Free $\mathrm{Et}_{2} \mathrm{O}$ was observed by ${ }^{1} \mathrm{H}$ NMR and ${ }^{13} \mathrm{C}\left\{{ }^{1} \mathrm{H}\right\}$ NMR because of the coordination of THF- $\mathrm{d}_{8}$ to potassium instead of $\mathrm{Et}_{2} \mathrm{O}$.

\section{Synthesis of 3.}

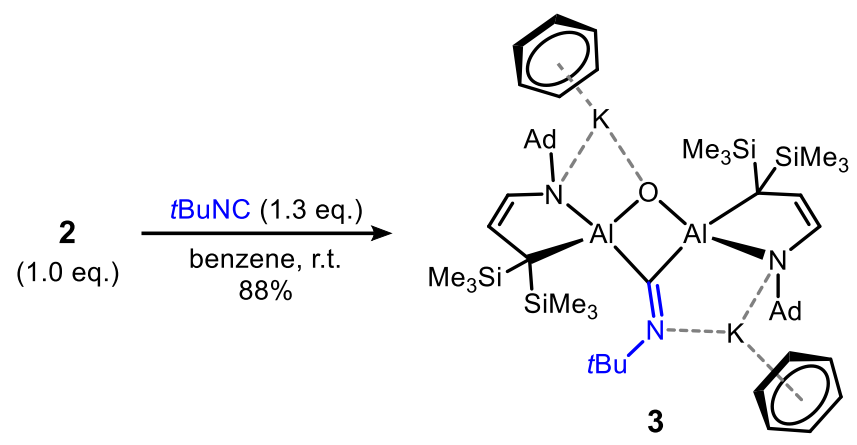

To a suspension of $2(20.0 \mathrm{mg}, 0.021 \mathrm{mmol})$ in benzene $(1.0 \mathrm{~mL})$ was added tert-butyl isocyanide $(3.0 \mu \mathrm{L}, 0.027 \mathrm{mmol})$. The mixture was stirred at room temperature. The solution color changed to orange. After 30 minutes, all volatiles were removed under vacuum to afford 3 as an orange solid (19.3 mg, 88\%). Single crystals suitable for X-ray diffraction analysis were obtained by recrystallization from a saturated benzene solution of $\mathbf{3}$ at room temperature.

M.p.: 98-101 ${ }^{\circ} \mathrm{C}$ (dec.); ${ }^{1} \mathrm{H}$ NMR (C6 6 6, $\left.400 \mathrm{MHz}, 298 \mathrm{~K}\right): \delta 6.59$ (d, J = $\left.6.0 \mathrm{~Hz}, 1 \mathrm{H}, \mathrm{CH}\right), \delta$ $6.55(\mathrm{~d}, J=5.6 \mathrm{~Hz}, 1 \mathrm{H}, \mathrm{CH}), 4.62(\mathrm{~d}, J=6.0 \mathrm{~Hz}, 1 \mathrm{H}, \mathrm{CH}), 4.44(\mathrm{~d}, J=6.0 \mathrm{~Hz}, 1 \mathrm{H}, \mathrm{CH}), 2.18$ (br, 3H, Ad-CH), 2.11 (br, 3H, Ad-CH), 1.91-1.84 (m, 3H, Ad-CH2), 1.84-1.77 (m, 3H, Ad-CH2), 1.77-1.62 (m, 12H, Ad-CH2), 1.62-1.54 (m, 3H, Ad-CH2), 1.52-1.45 (m, 3H, Ad-CH2), 1.35 (s, $\left.9 \mathrm{H}, \mathrm{C}\left(\mathrm{CH}_{3}\right)_{3}\right), 0.52\left(\mathrm{~s}, 9 \mathrm{H}, \mathrm{Si}-\mathrm{CH}_{3}\right), 0.48\left(\mathrm{~s}, 9 \mathrm{H}, \mathrm{Si}-\mathrm{CH}_{3}\right), 0.38\left(\mathrm{~s}, 9 \mathrm{H}, \mathrm{Si}-\mathrm{CH}_{3}\right), 0.20$ (s, 9H, Si- 
$\left.\mathrm{CH}_{3}\right) ;{ }^{13} \mathrm{C}\left\{{ }^{1} \mathrm{H}\right\}$ NMR $\left(\mathrm{C}_{6} \mathrm{D}_{6}, 100 \mathrm{MHz}, 298 \mathrm{~K}\right): \delta 139.3(\mathrm{CH}), 138.0(\mathrm{CH}), 99.7(\mathrm{CH}), 97.9(\mathrm{CH})$, $59.9\left(\mathrm{tBu}-\mathrm{C}\left(\mathrm{CH}_{3}\right)_{3}\right), 52.4(\mathrm{Ad}-\mathrm{qC}), 51.4(\mathrm{Ad}-\mathrm{qC}), 45.6\left(\mathrm{Ad}-\mathrm{CH}_{2}\right), 45.4\left(\mathrm{Ad}-\mathrm{CH}_{2}\right), 37.3\left(\mathrm{Ad}-\mathrm{CH}_{2}\right)$, $37.1\left(\mathrm{Ad}-\mathrm{CH}_{2}\right), 30.6(\mathrm{Ad}-\mathrm{CH}), 30.5(\mathrm{Ad}-\mathrm{CH}), 30.0\left(t \mathrm{Bu}-\mathrm{C}\left(\mathrm{CH}_{3}\right)_{3}\right), 16.5\left(\mathrm{AlC}_{3} \mathrm{~N}-\mathrm{qC}\right), 13.9$ $\left(\mathrm{AlC}_{3} \mathrm{~N}-\mathrm{qC}\right), 4.87\left(\mathrm{Si}-\mathrm{CH}_{3}\right), 4.26\left(\mathrm{Si}-\mathrm{CH}_{3}\right), 3.35\left(\mathrm{Si}-\mathrm{CH}_{3}\right), 2.68\left(\mathrm{Si}-\mathrm{CH}_{3}\right) ;{ }^{27} \mathrm{Al}\left\{{ }^{1} \mathrm{H}\right\}$ NMR $\left(\mathrm{C}_{6} \mathrm{D}_{6}\right.$, $104 \mathrm{MHz}, 298 \mathrm{~K}): \delta 122.2(\mathrm{br}) ;{ }^{29} \mathrm{Si}\left\{{ }^{1} \mathrm{H}\right\}$ NMR $\left(\mathrm{C}_{6} \mathrm{D}_{6}, 80 \mathrm{MHz}, 298 \mathrm{~K}\right) \delta-3.04,-3.52,-6.51$, -7.43; FT-IR (solid, cm-1): 2891, 2838, 1561, 1443, 1370, 1344, 1303, 1266, 1237, 1216, 1163, 1098, 1083, 943, 899, 859, 822, 778, 757, 742, 699, 685, 660, 626; UV-vis (THF): $\lambda_{\max }$ $=511 \mathrm{~nm}$; HRMS $\left(\mathrm{ESI}^{-}\right): \mathrm{m} / \mathrm{z}$ calcd for $\mathrm{C}_{43} \mathrm{H}_{79} \mathrm{~N}_{3} \mathrm{OAl}{ }_{2} \mathrm{Si}_{4} \mathrm{~K}\left[M-K\left(\mathrm{C}_{6} \mathrm{H}_{6}\right)_{2}\right]^{-} 858.4568$, found 858.4554; Anal. Calc. for $\mathrm{C}_{55} \mathrm{H}_{91} \mathrm{Al}_{2} \mathrm{~K}_{2} \mathrm{~N}_{3} \mathrm{OSi} 4$ : C $62.63 \%$, H 8.70\%, N 3.98\%, found: C 62.79\%, H $8.75 \%$, N 3.93\%.

The correlation $\left({ }^{3} \mathrm{~J}\right)$ between the quaternary carbon in the $\mathrm{AlC}_{3} \mathrm{~N}$ ring and the proton in TMS group was confirmed by ${ }^{1} \mathrm{H}-{ }^{13} \mathrm{C}$ HMBC NMR. The quaternary carbon in the $\mathrm{Al}_{2} \mathrm{OC}$ ring was not detected by ${ }^{13} \mathrm{C}\left\{{ }^{1} \mathrm{H}\right\}$ NMR because of the coupling with quadrupolar $\mathrm{Al}$.

\section{Synthesis of 4}

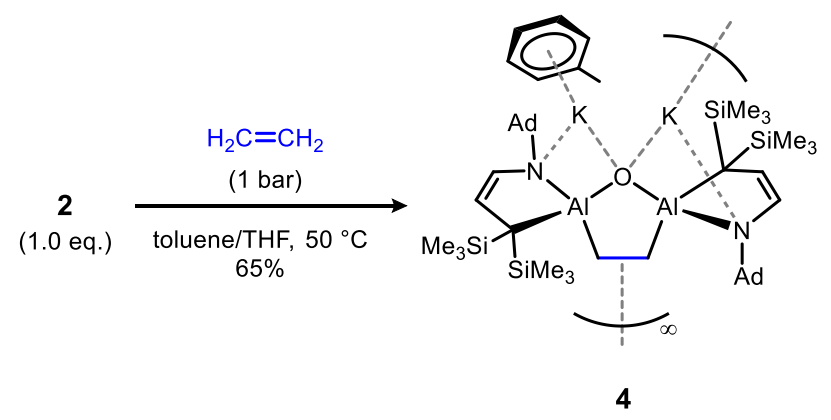

A solution of $2(20.0 \mathrm{mg}, 0.021 \mathrm{mmol})$ in toluene/THF $(10 / 1)(0.75 \mathrm{~mL})$ was degassed using a freeze-pump-thaw method and refilled with ethylene gas ( 1 bar) at room temperature. The mixture was heated at $50{ }^{\circ} \mathrm{C}$ for 12 hours and then allowed to cool to room temperature. The resulting solution was concentrated to ca. $0.2 \mathrm{~mL}$ by slow diffusion, affording colorless crystals suitable for X-ray diffraction analysis. The mother liquid was removed, and the colorless crystals were washed with hexane $(0.2 \mathrm{~mL} \times 3)$, and dried under vacuum to afford 4 as a colorless solid (12.7 mg, 65\%).

M.p.: $243-245{ }^{\circ} \mathrm{C}$ (dec.); ${ }^{1} \mathrm{H}$ NMR (THF-d8, $400 \mathrm{MHz}, 298 \mathrm{~K}$ ): $\delta 6.51$ (d, J = 5.6 Hz, 2H, CH), $3.97(\mathrm{~d}, J=6.0 \mathrm{~Hz}, 2 \mathrm{H}, \mathrm{CH}), 1.99(\mathrm{br}, 6 \mathrm{H}, \mathrm{Ad}-\mathrm{CH}), 1.95-1.88\left(\mathrm{~m}, 6 \mathrm{H}, \mathrm{Ad}-\mathrm{CH}_{2}\right), 1.83-1.76(\mathrm{~m}$, $\left.6 \mathrm{H}, \mathrm{Ad}-\mathrm{CH}_{2}\right), 1.73-1.66\left(\mathrm{~m}, 6 \mathrm{H}, \mathrm{Ad}-\mathrm{CH}_{2}\right), 1.60-1.52\left(\mathrm{~m}, 6 \mathrm{H}, \mathrm{Ad}-\mathrm{CH}_{2}\right), 0.08\left(\mathrm{~s}, 2 \mathrm{H}, \mathrm{Si}-\mathrm{CH}_{3}\right.$ 
and $\mathrm{AlCH} \mathrm{CH}_{2} \mathrm{Al}$ ), -0.09 (s, 20H, Si-CH and $\mathrm{AlCH} 2 \mathrm{CH}_{2} \mathrm{Al}$ ); ${ }^{13} \mathrm{C}\left\{{ }^{1} \mathrm{H}\right\} \mathrm{NMR}$ (THF-d8, $100 \mathrm{MHz}$, $298 \mathrm{~K}): \delta 139.7(\mathrm{CH}), 95.4(\mathrm{CH}), 51.6(\mathrm{Ad}-\mathrm{qC}), 45.5\left(\mathrm{Ad}-\mathrm{CH}_{2}\right), 38.1\left(\mathrm{Ad}-\mathrm{CH}_{2}\right), 31.4(\mathrm{Ad}-\mathrm{CH})$, $13.0\left(\mathrm{AlC}_{3} \mathrm{~N}-\mathrm{q} C\right), 10.8$ (br, AlCCAI), $3.83\left(\mathrm{Si}-\mathrm{CH}_{3}\right), 2.42\left(\mathrm{Si}-\mathrm{CH}_{3}\right) ;{ }^{27} \mathbf{A l}\left\{{ }^{1} \mathrm{H}\right\} \mathbf{N M R}\left(\mathrm{THF}-\mathrm{d}_{8}, 104\right.$ MHz, $298 \mathrm{~K}$ ): $\delta 141.7$ (br); ${ }^{29} \mathrm{Si}\left\{{ }^{1} \mathrm{H}\right\}$ NMR (THF-d8, $80 \mathrm{MHz}, 298 \mathrm{~K}$ ) $\delta-6.67,-7.29$; FT-IR (solid, $\mathrm{cm}^{-1}$ ): 2901, 2847, 2762, 1567, 1494, 1450, 1374, 1308, 1238, 1217, 1149, 1099, 1081, 947, 951, 906, 888, 860, 834, 821, 804, 781, 759, 745, 719, 696, 661; HRMS (ESI-): m/z calcd for $\mathrm{C}_{40} \mathrm{H}_{74} \mathrm{~N}_{2} \mathrm{OAl}_{2} \mathrm{Si}_{4} \mathrm{~K} \quad[M-K \cdot \text { toluene }]^{-}$803.4146, found 803.4165; Anal. Calc. for $\mathrm{C}_{47} \mathrm{H}_{82} \mathrm{Al}_{2} \mathrm{~K}_{2} \mathrm{~N}_{2} \mathrm{OS} \mathrm{Oi}_{4}$ : C $60.33 \%, \mathrm{H} 8.83 \%$, N 2.99\%, found: C 60.15\%, H 8.85\%, N 2.83\%.

Free toluene was observed by ${ }^{1} \mathrm{H}$ NMR and ${ }^{13} \mathrm{C}\left\{{ }^{1} \mathrm{H}\right\}$ NMR because of the coordination of THF$\mathrm{d}_{8}$ to potassium instead of toluene. The direct correlation $\left({ }^{1} \mathrm{~J}\right)$ between ${ }^{1} \mathrm{H}\left(\mathrm{AICH} \mathrm{CH}_{2} \mathrm{Al}\right)$ and ${ }^{13} \mathrm{C}\left(\mathrm{AlCH}{ }_{2} \mathrm{CH}_{2} \mathrm{Al}\right)$ was confirmed by HMQC NMR. The correlation ( $\left.{ }^{3} \mathrm{~J}\right)$ between the quaternary carbon in the $\mathrm{AlC}_{3} \mathrm{~N}$ ring and the proton in TMS group was confirmed by ${ }^{1} \mathrm{H}-{ }^{13} \mathrm{C} \mathrm{HMBC}$ NMR.

\section{Synthesis of 5}

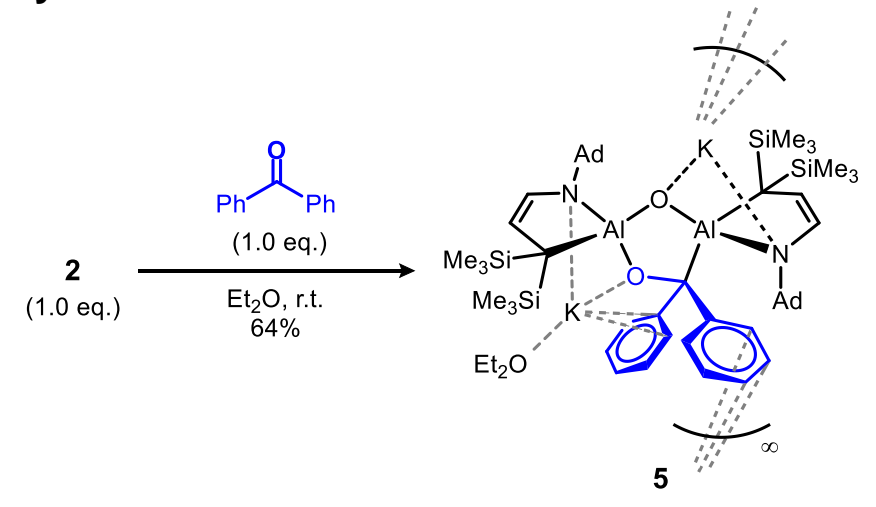

To a suspension of $2(20.0 \mathrm{mg}, 0.021 \mathrm{mmol})$ in $\mathrm{Et}_{2} \mathrm{O}(1.0 \mathrm{~mL})$ was added benzophenone (3.8 $\mathrm{mg}, 0.021 \mathrm{mmol})$. The mixture was stirred at room temperature for 5 minutes. The solution color changed to purple immediately after the treatment. After the stirring, the resulting solution was filtered and the residual solid was extracted with $\mathrm{Et}_{2} \mathrm{O}(1.5 \mathrm{~mL})$. To the combined Et2O solution was added hexane $(0.5 \mathrm{~mL})$, and concentrated to ca. $0.5 \mathrm{~mL}$ by slow diffusion, affording colorless crystals suitable for X-ray diffraction analysis. The colorless crystals were washed with hexane $(0.2 \mathrm{~mL} \times 3)$ and dried under vacuum to afford 5 as a white solid (14.2 $\mathrm{mg}$, $64 \%)$.

M.p.: $156-158{ }^{\circ} \mathrm{C}$ (dec.); ${ }^{1} \mathrm{H}$ NMR (THF-d8, $\left.400 \mathrm{MHz}, 298 \mathrm{~K}\right): \delta 7.90$ (d, J = $7.6 \mathrm{~Hz}, 2 \mathrm{H}$, Ph$\mathrm{CH}), 7.61(\mathrm{~d}, J=7.6 \mathrm{~Hz}, 2 \mathrm{H}, \mathrm{Ph}-\mathrm{CH}), 7.03(\mathrm{dd}, J=7.6 \mathrm{~Hz}$ and $7.6 \mathrm{~Hz}, 2 \mathrm{H}, \mathrm{Ph}-\mathrm{CH}), 6.90$ (dd, J $=7.6 \mathrm{~Hz}$ and $7.6 \mathrm{~Hz}, 2 \mathrm{H}, \mathrm{Ph}-\mathrm{CH}), 6.84(\mathrm{t}, J=7.2 \mathrm{~Hz}, 1 \mathrm{H}, \mathrm{Ph}-\mathrm{CH}), 6.76(\mathrm{t}, J=7.2 \mathrm{~Hz}, 1 \mathrm{H}, \mathrm{Ph}-$ $\mathrm{CH}), 6.45(\mathrm{~d}, J=6.0 \mathrm{~Hz}, 1 \mathrm{H}, \mathrm{CH}), 6.19(\mathrm{~d}, J=6.0 \mathrm{~Hz}, 1 \mathrm{H}, \mathrm{CH}), 4.07(\mathrm{~d}, J=6.0 \mathrm{~Hz}, 1 \mathrm{H}, \mathrm{CH})$, 
$4.01(\mathrm{~d}, J=6.4 \mathrm{~Hz}, 1 \mathrm{H}, \mathrm{CH}), 2.18(\mathrm{br}, 3 \mathrm{H}, \mathrm{Ad}-\mathrm{CH}), 2.05-1.98(\mathrm{~m}, 3 \mathrm{H}, \mathrm{Ad}-\mathrm{CH}$ ), 1.98-1.92 (m, $\left.3 \mathrm{H}, \mathrm{Ad}-\mathrm{CH}_{2}\right), 1.82-1.75\left(\mathrm{~m}, 3 \mathrm{H}, \mathrm{Ad}-\mathrm{CH}_{2}\right), 1.73$ (br, 3H, Ad-CH, overlap with a peak of THF-d7), 1.64-1.57 (m, 3H, Ad-CH2), 1.54-1.43 (m, 6H, Ad-CH2), 1.35-1.28 (m, 3H, Ad-CH2), 1.23$1.17\left(\mathrm{~m}, 3 \mathrm{H}, \mathrm{Ad}-\mathrm{CH}_{2}\right), 0.12$ (s, 9H, Si-CH3), 0.05 (s, 9H, Si-CH3), -0.06 (s, 9H, Si-CH3), -0.09 (s, 9H, Si-CH3); ${ }^{13} \mathrm{C}\left\{{ }^{1} \mathrm{H}\right\}$ NMR (THF-d8, $\left.100 \mathrm{MHz}, 298 \mathrm{~K}\right): \delta 154.1(\mathrm{Ph}-\mathrm{C}), 153.3(\mathrm{Ph}-\mathrm{C}), 138.7$ $(\mathrm{CH}), 137.5(\mathrm{CH}), 130.2(\mathrm{Ph}-\mathrm{C}), 128.7(\mathrm{Ph}-\mathrm{C}), 126.1(\mathrm{Ph}-\mathrm{C}), 125.8(\mathrm{Ph}-\mathrm{C}), 124.5(\mathrm{Ph}-\mathrm{C})$, $123.7(\mathrm{Ph}-\mathrm{C}), 97.8(\mathrm{CH}), 93.5(\mathrm{CH}), 78.5\left(\mathrm{OC}(\mathrm{Ph})_{2} \mathrm{Al}\right), 52.3(\mathrm{Ad}-\mathrm{q} C), 51.6(\mathrm{Ad}-\mathrm{qC}), 45.6(\mathrm{Ad}-$ $\left.\mathrm{CH}_{2}\right), 45.1\left(\mathrm{Ad}-\mathrm{CH}_{2}\right), 38.3\left(\mathrm{Ad}-\mathrm{CH}_{2}\right), 38.0\left(\mathrm{Ad}-\mathrm{CH}_{2}\right), 31.4(\mathrm{Ad}-\mathrm{CH}), 31.3(\mathrm{Ad}-\mathrm{CH}), 15.5\left(\mathrm{AlC}_{3} \mathrm{~N}-\right.$ $\mathrm{qC}), 11.0\left(\mathrm{AlC}_{3} \mathrm{~N}-\mathrm{qC}\right), 5.09\left(\mathrm{Si}-\mathrm{CH}_{3}\right), 4.98\left(\mathrm{Si}-\mathrm{CH}_{3}\right), 3.70\left(\mathrm{Si}-\mathrm{CH}_{3}\right), 3.40\left(\mathrm{Si}-\mathrm{CH}_{3}\right) ;{ }^{29} \mathrm{Si}\left\{{ }^{1} \mathrm{H}\right\}$ NMR (THF-d8, 80 MHz, 298 K) $\delta-3.45,-6.94,-8.16$; FT-IR (solid, cm ${ }^{-1}$ ): 2983, 2890, 2838, 1578, 1480, 1444, 1376, 1303, 1223, 1149, 1096, 1067, 951, 927, 867, 830, 783, 750, 712, $695,671,661,643,614$; HRMS $\left(\mathrm{ESI}^{-}\right): \mathrm{m} / \mathrm{z}$ calcd for $\mathrm{C}_{51} \mathrm{H}_{80} \mathrm{~N}_{2} \mathrm{O}_{2} \mathrm{Al}_{2} \mathrm{Si}_{4} \mathrm{~K}\left[\mathrm{M}-\mathrm{K} \cdot \mathrm{OEt_{2 }}\right]^{-}$ 957.4565, found 957.4588; Anal. Calc. for $\mathrm{C}_{55} \mathrm{H}_{90} \mathrm{Al}_{2} \mathrm{~K}_{2} \mathrm{~N}_{2} \mathrm{O}_{3} \mathrm{Si}_{4}$ : C 61.63\%, H 8.46\%, N 2.61\%, found: C $61.58 \%, \mathrm{H} 8.71 \%, \mathrm{~N} 2.67 \%$.

Free $\mathrm{Et}_{2} \mathrm{O}$ was observed by ${ }^{1} \mathrm{H}$ NMR and ${ }^{13} \mathrm{C}\left\{{ }^{1} \mathrm{H}\right\}$ NMR because of the coordination of THF- $\mathrm{d}_{8}$ to potassium instead of $\mathrm{Et}_{2} \mathrm{O}$. The correlation $\left({ }^{3} \mathrm{~J}\right.$ ) between the quaternary carbon in the $\mathrm{AlC}_{3} \mathrm{~N}$ ring and the proton in TMS group was confirmed by ${ }^{1} \mathrm{H}-{ }^{13} \mathrm{C} \mathrm{HMBC}$ NMR. The Al signal was not detected by ${ }^{27} \mathrm{Al}\left\{{ }^{1} \mathrm{H}\right\}$ NMR. Only three singlet peaks were observed in the ${ }^{29} \mathrm{Si}\left\{{ }^{1} \mathrm{H}\right\} \mathrm{NMR}$ spectrum, probably due to the overlap of two singlets at $-3.45 \mathrm{ppm}$.

\section{Synthesis of 6}

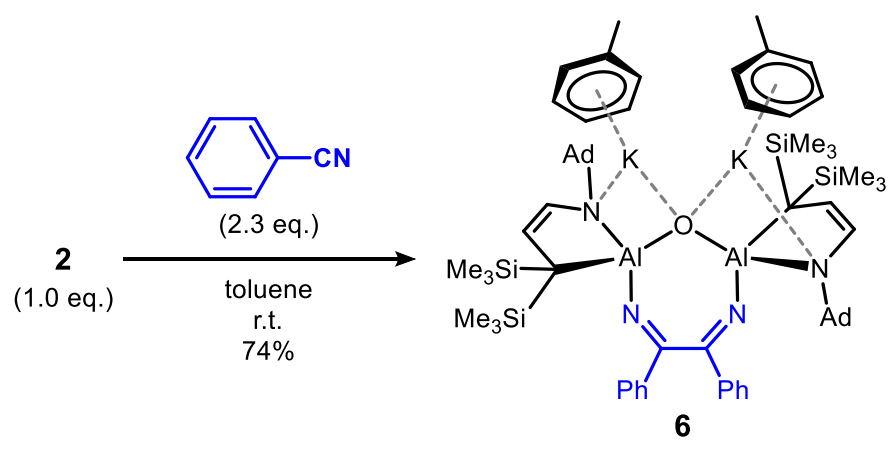

To a suspension of $2(20.0 \mathrm{mg}, 0.021 \mathrm{mmol})$ in toluene $(1.0 \mathrm{~mL})$ was added benzonitrile $(5.0$ $\mu \mathrm{L}, 0.048 \mathrm{mmol})$. The solution color changed to red immediately after the treatment. The mixture was stirred at room temperature for 5 minutes, and hexane $(1 \mathrm{~mL})$ was added to the resulting solution. The toluene/hexane solution was concentrated to ca. $0.5 \mathrm{~mL}$ by slow diffusion, affording red crystals suitable for X-ray diffraction analysis. The red crystals were 
washed with hexane $(0.5 \mathrm{~mL} \times 3)$ and dried under vacuum to afford 6 as an orange solid (18.6 mg, 74\%).

M.p.: $276-278{ }^{\circ} \mathrm{C}$ (dec.); ${ }^{1} \mathrm{H}$ NMR (THF-d8, $400 \mathrm{MHz}, 298 \mathrm{~K}$ ): $\delta 7.69$ (d, $J=6.8 \mathrm{~Hz}, 4 \mathrm{H}, \mathrm{Ph}-$ $\mathrm{CH}, 6.96(\mathrm{dd}, J=6.8 \mathrm{~Hz}$ and $6.8 \mathrm{~Hz}, 4 \mathrm{H}, \mathrm{Ph}-\mathrm{CH}), 6.89(\mathrm{t}, J=6.8 \mathrm{~Hz}, 2 \mathrm{H}, \mathrm{Ph}-\mathrm{CH}), 6.53(\mathrm{~d}, J=$ $6.0 \mathrm{~Hz}, 2 \mathrm{H}, \mathrm{CH}), 4.16(\mathrm{~d}, \mathrm{~J}=6.0 \mathrm{~Hz}, 2 \mathrm{H}, \mathrm{CH}), 1.68-1.62\left(\mathrm{~m}, 6 \mathrm{H}, \mathrm{Ad}-\mathrm{CH}_{2}\right), 1.60-1.54(\mathrm{~m}, 6 \mathrm{H}$, Ad-CH $\left.H_{2}\right), 1.42$ (br, 6H, Ad-CH), 1.39-1.33 (m, 6H, Ad-CH2), 1.21-1.14 (m, 6H, Ad-CH2), 0.14 (s, 18H, Si-CH $\left.H_{3}\right), 0.09$ (s, 18H, Si-CH$\left.)_{3}\right) ;{ }^{13} \mathrm{C}\left\{{ }^{1} \mathrm{H}\right\}$ NMR (THF-d8, $100 \mathrm{MHz}, 298 \mathrm{~K}$ ): $\delta 169.5$ (NCCN), 144.8 (Ph-C), $138.9(\mathrm{CH}), 129.5$ (Ph-C), $126.7(\mathrm{Ph}-\mathrm{C}), 126.2(\mathrm{Ph}-\mathrm{C}), 96.6(\mathrm{CH}), 51.6$ ( $\mathrm{Ad}-\mathrm{qC}), 45.6\left(\mathrm{Ad}-\mathrm{CH}_{2}\right), 37.7\left(\mathrm{Ad}-\mathrm{CH}_{2}\right), 31.0(\mathrm{Ad}-\mathrm{CH}), 12.6\left(\mathrm{AlC}_{3} \mathrm{~N}-\mathrm{qC}\right), 4.15\left(\mathrm{Si}-\mathrm{CH}_{3}\right), 2.77$ $\left(\mathrm{Si}-\mathrm{CH}_{3}\right) ;{ }^{27} \mathbf{A l}\left\{{ }^{1} \mathrm{H}\right\}$ NMR (THF-d8, $104 \mathrm{MHz}, 298 \mathrm{~K}$ ): $\delta 111.9$ (br); ${ }^{29} \mathrm{Si}\left\{{ }^{1} \mathrm{H}\right\} \mathbf{N M R}$ (THF-d8, 80 MHz, 298 K) $\delta-6.01,-76.04$; FT-IR (solid, $\mathrm{cm}^{-1}$ ): 2907, 1644, 1616, 1557, 1445, 1373, 1307, $1229,1216,1153,1100,1006,974,949,884,835,825,747,701,660,622,611$; UV-vis (THF): $\lambda_{\max }=520 \mathrm{~nm}$; HRMS $\left(\mathrm{ESI}^{-}\right): \mathrm{m} / \mathbf{z}$ calcd for $\left.\mathrm{C}_{52} \mathrm{H}_{80} \mathrm{~N}_{4} \mathrm{OAl}_{2} \mathrm{Si}_{4} \mathrm{~K}[\mathrm{M}-\mathrm{K} \text { (toluene) }]_{2}\right]^{-}$ 981.4677, found 981.4676; Anal. Calc. for $\mathrm{C}_{60} \mathrm{H}_{96} \mathrm{Al}_{2} \mathrm{~K}_{2} \mathrm{~N}_{4} \mathrm{OSi}_{4}$ : C 65.73\%, H 8.02\%, N 4.65\%, found: C $65.47 \%, \mathrm{H} 8.30 \%, \mathrm{~N} 4.76 \%$.

As sample of 6 for the NMR measurements was synthesized in toluene-d8, no peak corresponding to toluene was observed by ${ }^{1} \mathrm{H}$ NMR and ${ }^{13} \mathrm{C}\left\{{ }^{1} \mathrm{H}\right\}$ NMR. The correlation $\left({ }^{3} \mathrm{~J}\right)$ between the quaternary carbon in the $\mathrm{AIC}_{3} \mathrm{~N}$ ring and the proton in TMS group was confirmed by ${ }^{1} \mathrm{H}-{ }^{13} \mathrm{C}$ HMBC NMR. 


\section{Synthesis of 7}

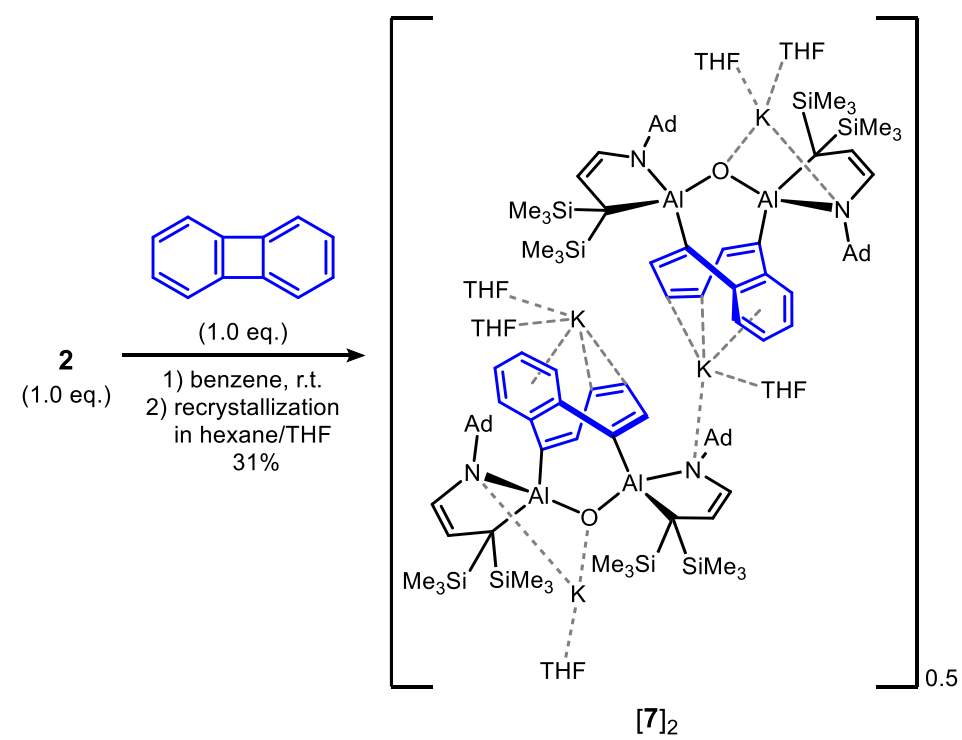

To a suspension of $2(20.0 \mathrm{mg}, 0.021 \mathrm{mmol})$ in benzene $(1.5 \mathrm{~mL})$ was added biphenylene (3.2 $\mathrm{mg}, 0.021 \mathrm{mmol})$. The mixture was stirred at room temperature for 15 minutes. After the stirring, the resulting solution was filtered to remove an insoluble solid. The resulting solution was left to stand in a small vial at room temperature for 1 day, affording a white powder on the bottom of the vial. The mother liquid was removed, and the white powder was washed with hexane $(0.2 \mathrm{~mL} \times 3)$ and dried under vacuum. The resulting white powder was recrystallized in hexane/THF (10/1) at room temperature to form 6 as a colorless crystal suitable for X-ray diffraction analysis. The colorless crystals were washed with hexane $(0.2 \mathrm{~mL} \times 3)$ and dried under vacuum to afford 7 as a white solid $(7.6 \mathrm{mg}, 31 \%)$.

M.p.: $250.0-253.0{ }^{\circ} \mathrm{C}$ (dec.); ${ }^{1} \mathrm{H}$ NMR (THF-d8, $400 \mathrm{MHz}, 298 \mathrm{~K}$ ): $\delta 6.75$ (ddd, $J=7.2 \mathrm{~Hz}, 7.2$ $\mathrm{Hz}$, and $1.2 \mathrm{~Hz}, 1 \mathrm{H}, \mathrm{Ar}-\mathrm{CH}), 6.71(\mathrm{~d}, J=7.2 \mathrm{~Hz}, 1 \mathrm{H}, \mathrm{Ar}-\mathrm{CH}), 6.67-6.58(\mathrm{~m}, 3 \mathrm{H}, \mathrm{CH}$ and Ar$\mathrm{CH}), 6.56(\mathrm{~d}, J=6.0 \mathrm{~Hz}, \mathrm{CH}), 6.38(\mathrm{br}, 1 \mathrm{H}, \mathrm{CH}), 6.16(\mathrm{br}, 1 \mathrm{H}, \mathrm{CH}), 5.89(\mathrm{dd}, J=10.8 \mathrm{~Hz}$ and $2.0 \mathrm{~Hz}, 1 \mathrm{H}, \mathrm{CH}), 5.81(\mathrm{dd}, J=10.8 \mathrm{~Hz}$ and $2.0 \mathrm{~Hz}, 1 \mathrm{H}, \mathrm{CH}), 4.19(\mathrm{~d}, J=6.0 \mathrm{~Hz}, \mathrm{CH}), 3.93(\mathrm{~d}$, $J=6.0 \mathrm{~Hz}, \mathrm{CH}), 2.01(\mathrm{br}, 3 \mathrm{H}, \mathrm{Ad}-\mathrm{CH}), 1.95-1.83\left(\mathrm{~m}, 6 \mathrm{H}, \mathrm{Ad}-\mathrm{CH}_{2}\right), 1.80-1.63(\mathrm{~m}, 9 \mathrm{H}, \mathrm{Ad}-\mathrm{CH}$ and Ad- $\mathrm{CH}_{2}$, overlap with a peak of THF-d7), $1.60-1.50\left(\mathrm{~m}, 6 \mathrm{H}, \mathrm{Ad}-\mathrm{CH}_{2}\right), 1.40-1.33(\mathrm{~m}, 3 \mathrm{H}$, Ad- $\mathrm{CH}_{2}$ ), 1.17-1.09 (m, 3H, Ad-CH2), 0.14 (s, 9H, Si-CH $\mathrm{CH}_{3}, 0.08$ (s, 9H, Si-CH $\mathrm{CH}_{3}, 0.04$ (s, 9H, $\left.\mathrm{Si}-\mathrm{CH}_{3}\right), 0.00$ (s, 9H, Si-CH3); ${ }^{13} \mathrm{C}\left\{{ }^{1} \mathrm{H}\right\}$ NMR (THF-d8, $100 \mathrm{MHz}, 298 \mathrm{~K}$ ): $\delta 152.9$ (Ar-qC), 152.2 (Ar-qC), 140.4 (Al[N(Ad) $\left.\left.\mathrm{CHCHC}(\mathrm{TMS})_{2}\right]\right), 139.9$ (Al[N(Ad) $\left.\left.\mathrm{CHCHC}(\mathrm{TMS})_{2}\right]\right), 137.5$ (AlC=CH$\mathrm{CH}), 134.9(\mathrm{AlC}=\mathrm{CH}-\mathrm{CH}), 134.0(\mathrm{AlC}=\mathrm{CH}-\mathrm{CH}), 133.9(\mathrm{AlC}=\mathrm{CH}-\mathrm{CH}), 125.6(\mathrm{Ar}-\mathrm{CH}), 122.9$

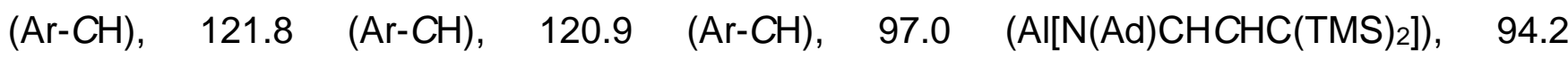
(Al[N(Ad) $\left.\left.\mathrm{CHCHC}(\mathrm{TMS})_{2}\right]\right), 53.2(\mathrm{Ad}-\mathrm{qC}), 52.7$ (Ad-qC), $44.6\left(\mathrm{Ad}-\mathrm{CH}_{2}\right), 44.4\left(\mathrm{Ad}-\mathrm{CH}_{2}\right), 38.1$ 
(Ad- $\left.\mathrm{CH}_{2}\right), 37.3\left(\mathrm{Ad}-\mathrm{CH}_{2}\right), 31.4(\mathrm{Ad}-\mathrm{CH}), 31.2(\mathrm{Ad}-\mathrm{CH}), 14.4\left(\mathrm{AlC}_{3} \mathrm{~N}-\mathrm{qC}\right), 13.6\left(\mathrm{AlC}_{3} \mathrm{~N}-\mathrm{qC}\right), 3.81$ $\left(\mathrm{Si}-\mathrm{CH}_{3}\right), 3.57\left(\mathrm{Si}-\mathrm{CH}_{3}\right), 3.41\left(\mathrm{Si}-\mathrm{CH}_{3}\right), 3.27\left(\mathrm{Si}-\mathrm{CH}_{3}\right) ;{ }^{27} \mathrm{Al}\left\{{ }^{1} \mathrm{H}\right\}$ NMR $\left(\mathrm{THF}-\mathrm{d}_{8}, 104 \mathrm{MHz}, 298\right.$ K): $\delta 120.8$ (br); ${ }^{29} \mathrm{Si}\left\{{ }^{1} \mathrm{H}\right\}$ NMR (THF-d $\left.8,80 \mathrm{MHz}, 298 \mathrm{~K}\right) \delta-4.56,-4.75,-7.00,-10.7$; FT-IR (solid, $\mathrm{cm}^{-1}$ ): 2967, 2894, 2836, 1563, 1443, 1374, 1303, 1266, 1222, 1149, 1097, 1047, 966, 946, 908, 881, 862, 828, 802, 774, 753, 732, 692, 657, 625; HRMS (ESI-): m/z calcd for $\mathrm{C}_{50} \mathrm{H}_{78} \mathrm{~N}_{2} \mathrm{OAl}_{2} \mathrm{Si}_{4} \mathrm{~K} \quad\left[M-K(T H F)_{3}\right]^{-} \quad 927.4459$, found 927.4468; Anal. Calc. for $\mathrm{C}_{62} \mathrm{H}_{102} \mathrm{Al}_{2} \mathrm{~K}_{2} \mathrm{~N}_{2} \mathrm{O}_{4} \mathrm{Si}_{4}$ : C 62.90\%, H 8.68\%, N 2.37\%, found: C 62.98\%, H 8.61\%, N 2.48\%.

The $\mathrm{sp}^{2}$ carbons next to $\mathrm{Al}$ were not detected by ${ }^{13} \mathrm{C}\left\{{ }^{1} \mathrm{H}\right\}$ NMR because of the coupling with quadrupolar Al. The correlation $\left({ }^{3} \mathrm{~J}\right)$ between the quaternary carbon in the $\mathrm{AlC}_{3} \mathrm{~N}$ ring and the proton in TMS group was confirmed by ${ }^{1} \mathrm{H}-{ }^{13} \mathrm{C}$ HMBC NMR.

\section{Synthesis of 8}

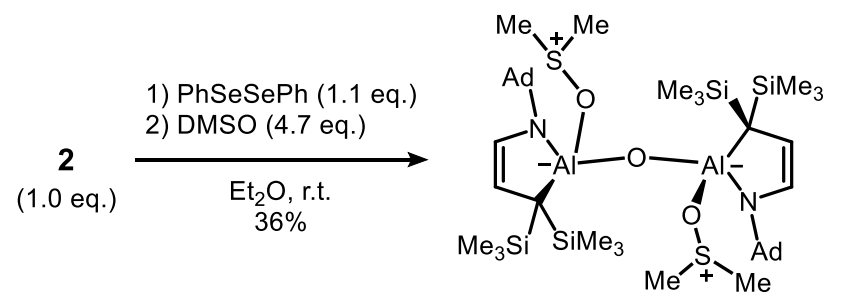

8

To a suspension of $2(20.0 \mathrm{mg}, 0.021 \mathrm{mmol})$ in $\mathrm{Et}_{2} \mathrm{O}(1.0 \mathrm{~mL})$ was added diphenyl diselenide (7.1 $\mathrm{mg}, 0.023 \mathrm{mmol})$, immediately producing white solid. The mixture was stirred for 5 minutes at room temperature, then volatiles were removed under vacuum. The residual solid was extracted with hexane $(1.0 \mathrm{~mL} \times 2)$ and the solvent was removed under vacuum to afford sticky colorless solid. $\mathrm{Et}_{2} \mathrm{O}(1.5 \mathrm{~mL})$ was added to the resulting solid, and the $\mathrm{Et}_{2} \mathrm{O}$ solution was treated with dimethyl sulfoxide $(7.0 \mu \mathrm{L}, 0.099 \mathrm{mmol})$, and the resulting solution was concentrated to ca. $0.5 \mathrm{~mL}$ by slow diffusion at room temperature, affording colorless crystals suitable for X-ray diffraction analysis. The colorless crystals were washed with hexane (1.0 $\mathrm{mL} \times 5)$ and dried under vacuum to afford 8 as a white solid (6.6 mg, $36 \%$ ).

M.p.: $176-177^{\circ} \mathrm{C}$ (dec.); ${ }^{1} \mathrm{H}$ NMR ( $\mathrm{C}_{6} \mathrm{D}_{6}, 400 \mathrm{MHz}, 298 \mathrm{~K}$ ): 6.63 (d, J=6.0 Hz, 2H, CH), 4.76 (d, J = 6.0 Hz, 2H, CH), 2.32-2.24 (br, 6H, Ad-CH), 2.09-1.98 (m, 12H, Ad-CH2), 2.01 (s, 6H, $\left.\mathrm{SCH}_{3}\right), 1.90-1.77\left(\mathrm{~m}, 12 \mathrm{H}, \mathrm{Ad}-\mathrm{CH}_{2}\right), 1.85$ (s, 6H, SCH$), 0.51$ (s, 18H, Si-CH3), 0.37 (s, 18H, $\left.\mathrm{Si}-\mathrm{CH}_{3}\right) ;{ }^{13} \mathrm{C}\left\{{ }^{1} \mathrm{H}\right\}$ NMR $\left(\mathrm{C}_{6} \mathrm{D}_{6}, 100 \mathrm{MHz}, 298 \mathrm{~K}\right): \delta 137.3(\mathrm{CH}), 98.7(\mathrm{CH}), 51.8(\mathrm{Ad}-\mathrm{qC}), 46.1$ (Ad- $\left.\mathrm{CH}_{2}\right), 37.7\left(\mathrm{Ad}-\mathrm{CH}_{2}\right), 36.3\left(\mathrm{SCH}_{3}\right), 36.1\left(\mathrm{SCH}_{3}\right), 30.7(\mathrm{Ad}-\mathrm{CH}), 4.54\left(\mathrm{Si}-\mathrm{CH}_{3}\right), 2.60(\mathrm{Si}-$ $\left.\mathrm{CH}_{3}\right) ;{ }^{29} \mathrm{Si}\left\{{ }^{1} \mathrm{H}\right\} \mathrm{NMR}\left(\mathrm{C}_{6} \mathrm{D}_{6}, 80 \mathrm{MHz}, 298 \mathrm{~K}\right) \delta-3.43,-6.52 ; \mathrm{FT}-\mathrm{IR}\left(\right.$ solid, $\left.\mathrm{cm}^{-1}\right): 2897,2845$, 
1552, 1446, 1370, 1359, 1308, 1271, 1238, 1214, 1173, 1099, 1084, 1057, 1017, 983, 955, 934, 883, 858, 831, 760, 691, 666, 616; HRMS $\left(\mathrm{ESI}^{+}\right): \mathrm{m} / \mathrm{z}$ calcd for $\mathrm{C}_{42} \mathrm{H}_{83} \mathrm{~N}_{2} \mathrm{O}_{3} \mathrm{Al}_{2} \mathrm{Si}_{4} \mathrm{~S}_{2}$ $[\mathrm{M}+\mathrm{H}]^{+}$893.4553, found 893.4555; Anal. Calc. for $\mathrm{C}_{42} \mathrm{H}_{82} \mathrm{Al}_{2} \mathrm{~N}_{2} \mathrm{O}_{3} \mathrm{~S}_{2} \mathrm{Si}_{4}$ : C 56.46\%, H 9.25\%, N 3.14\%, found: C $56.40 \%, \mathrm{H} 9.28 \%$, N $2.85 \%$.

The quaternary carbon next to Al was not detected by ${ }^{13} \mathrm{C}\left\{{ }^{1} \mathrm{H}\right\}$ NMR because of the coupling with quadrupolar Al. The Al signal was not detected by ${ }^{27} \mathrm{Al}\left\{{ }^{1} \mathrm{H}\right\}$ NMR. 


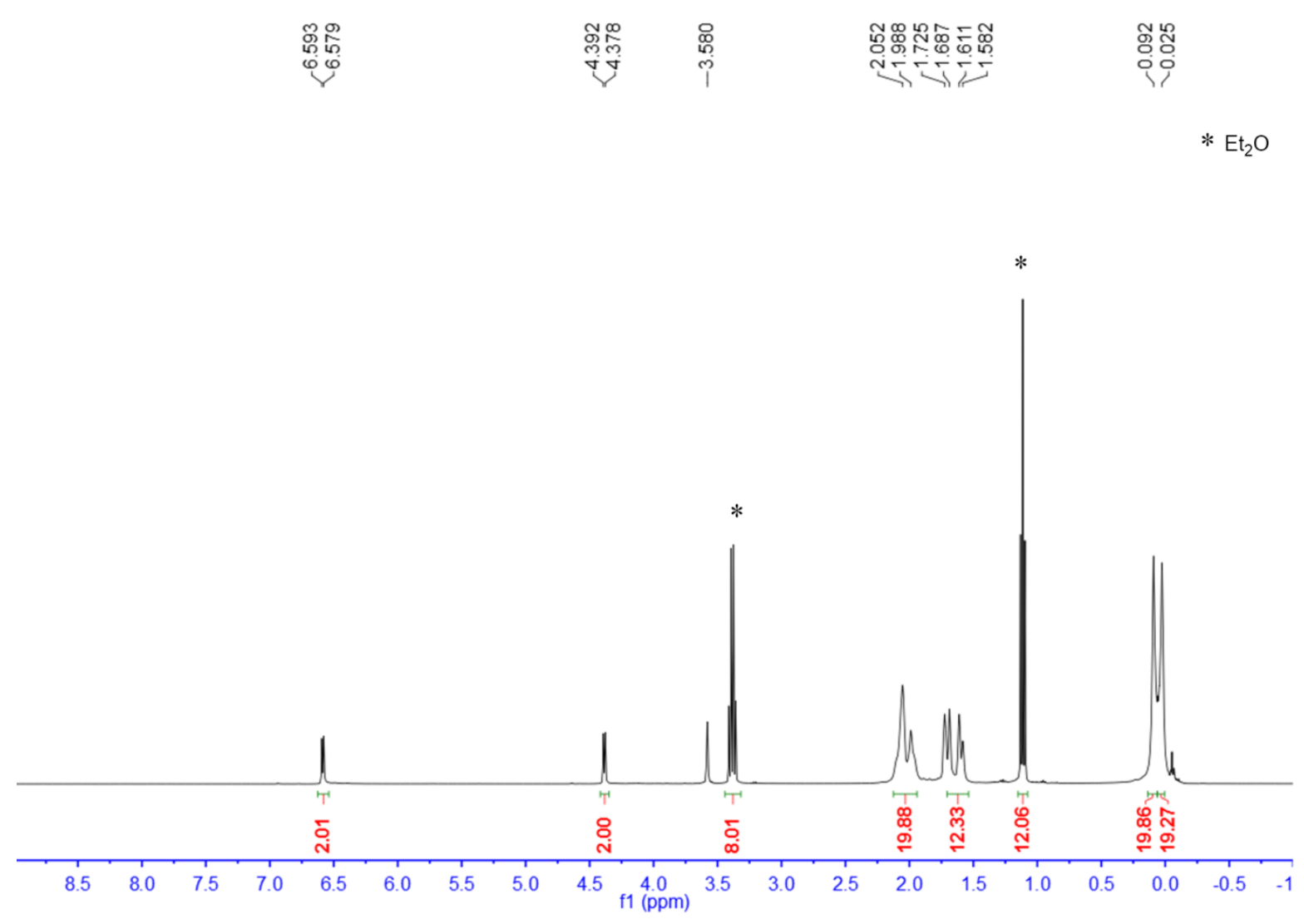

Figure S1. ${ }^{1} \mathrm{H}$ NMR spectrum of 2 in THF-d8.

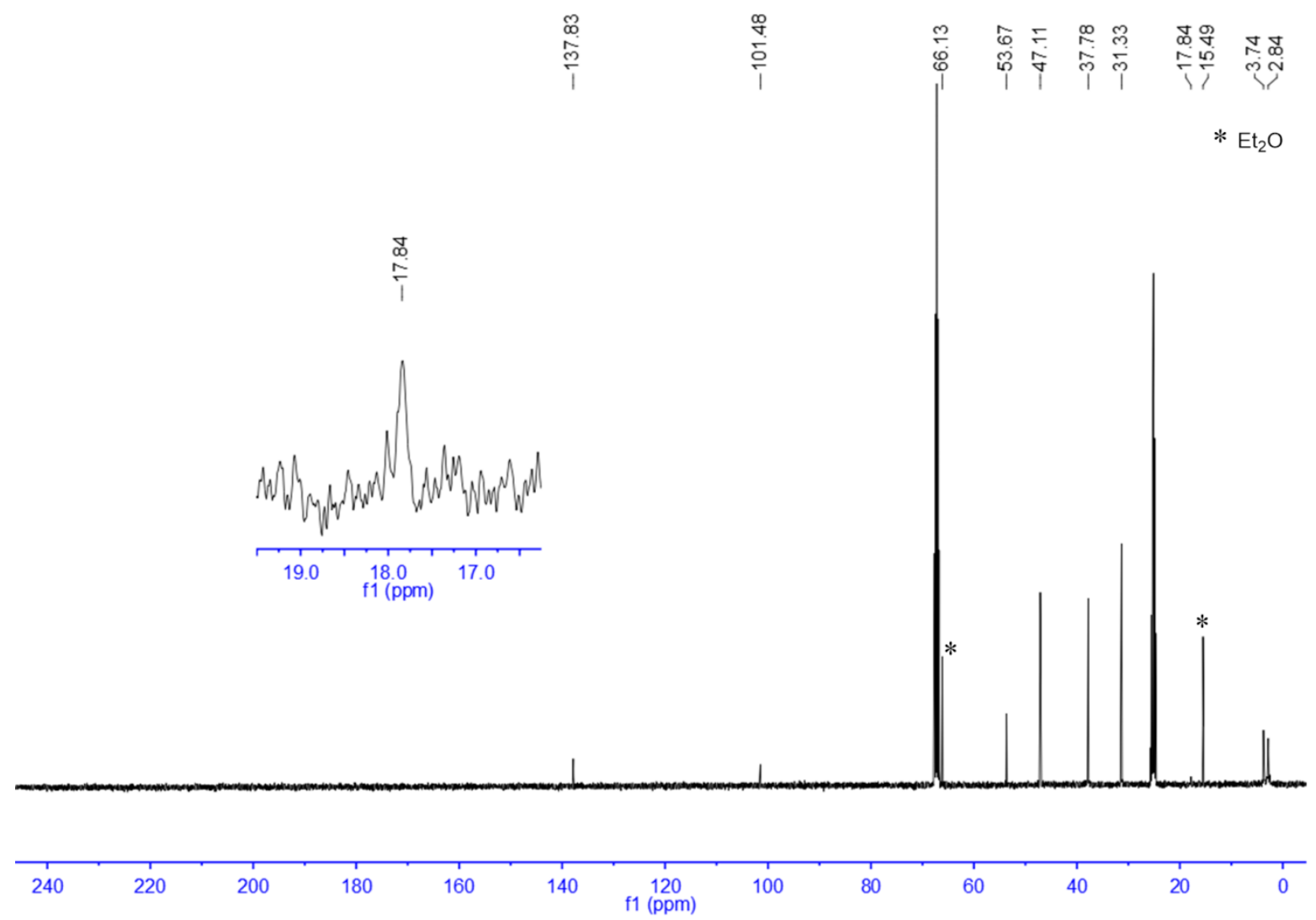

Figure S2. ${ }^{13} \mathrm{C}\left\{{ }^{1} \mathrm{H}\right\}$ NMR spectrum of 2 in THF-d8. 


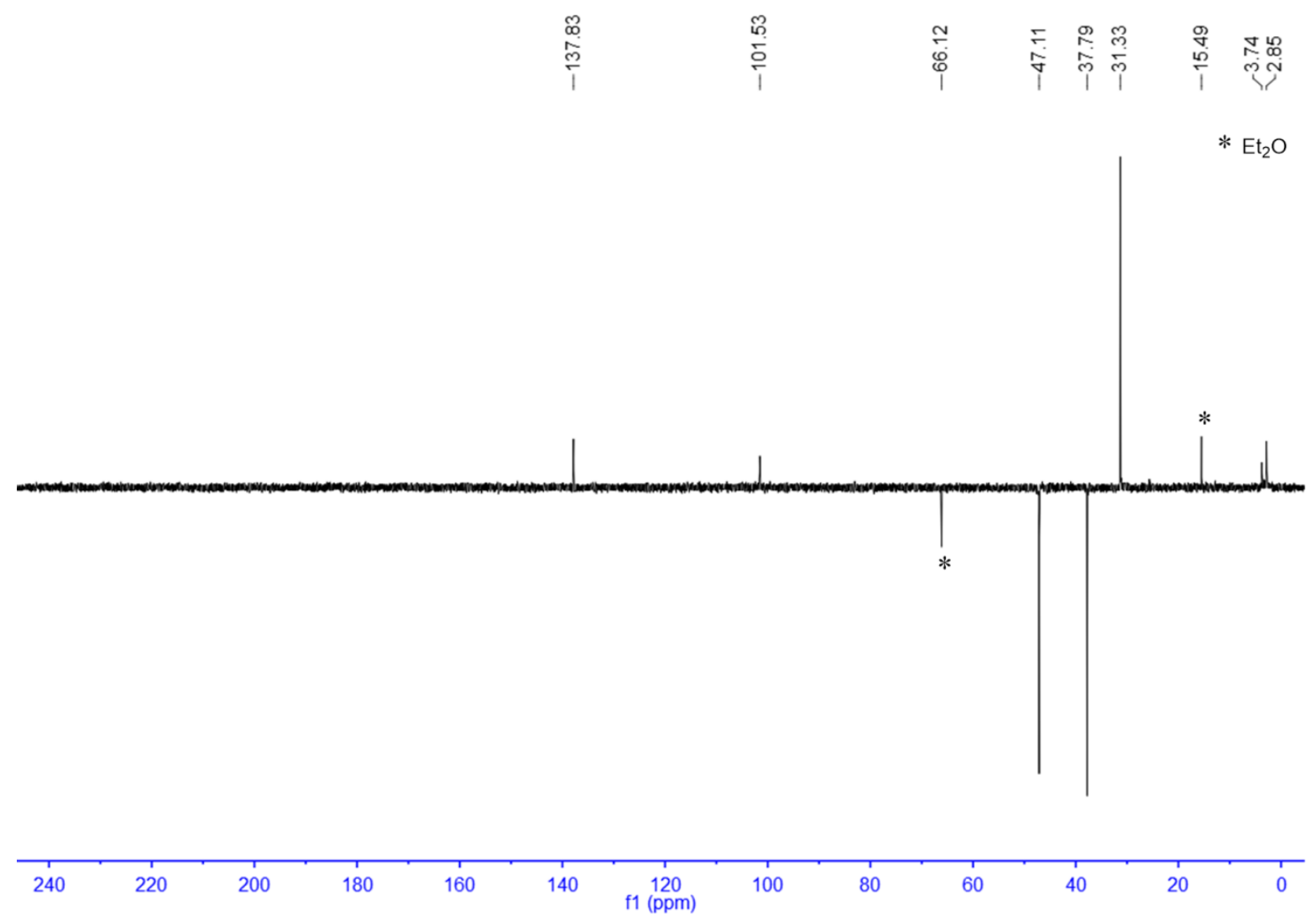

Figure S3. ${ }^{13} \mathrm{C}$ (DEPT 135) NMR spectrum of 2 in THF-d8.

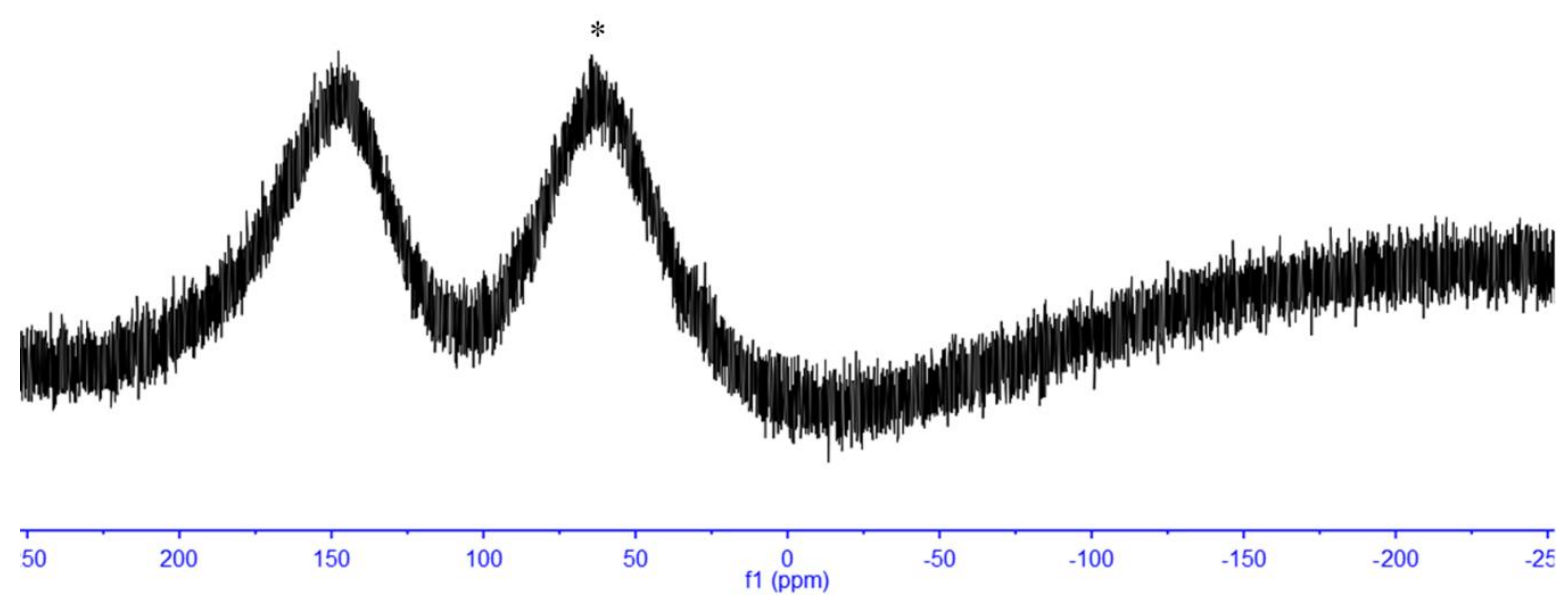

Figure S4. ${ }^{27} \mathrm{Al}\left\{{ }^{1} \mathrm{H}\right\}$ NMR spectrum of 2 in THF-d8. 


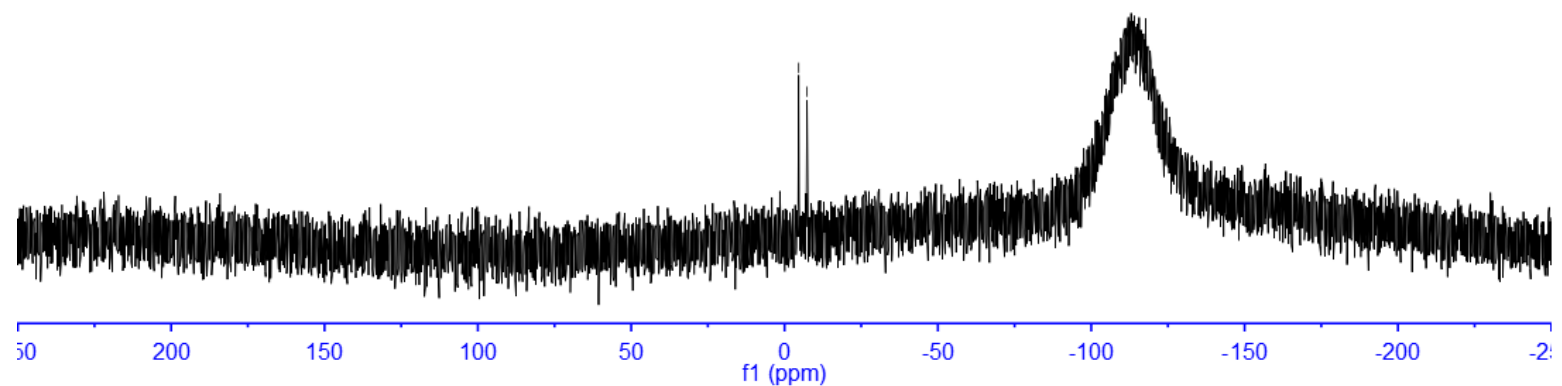

Figure S5. ${ }^{29} \mathrm{Si}\left\{{ }^{1} \mathrm{H}\right\}$ NMR spectrum of 2 in THF-d8.

\begin{tabular}{|c|c|c|}
\hline 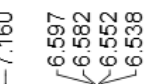 & 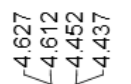 & 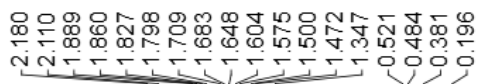 \\
\hline
\end{tabular}

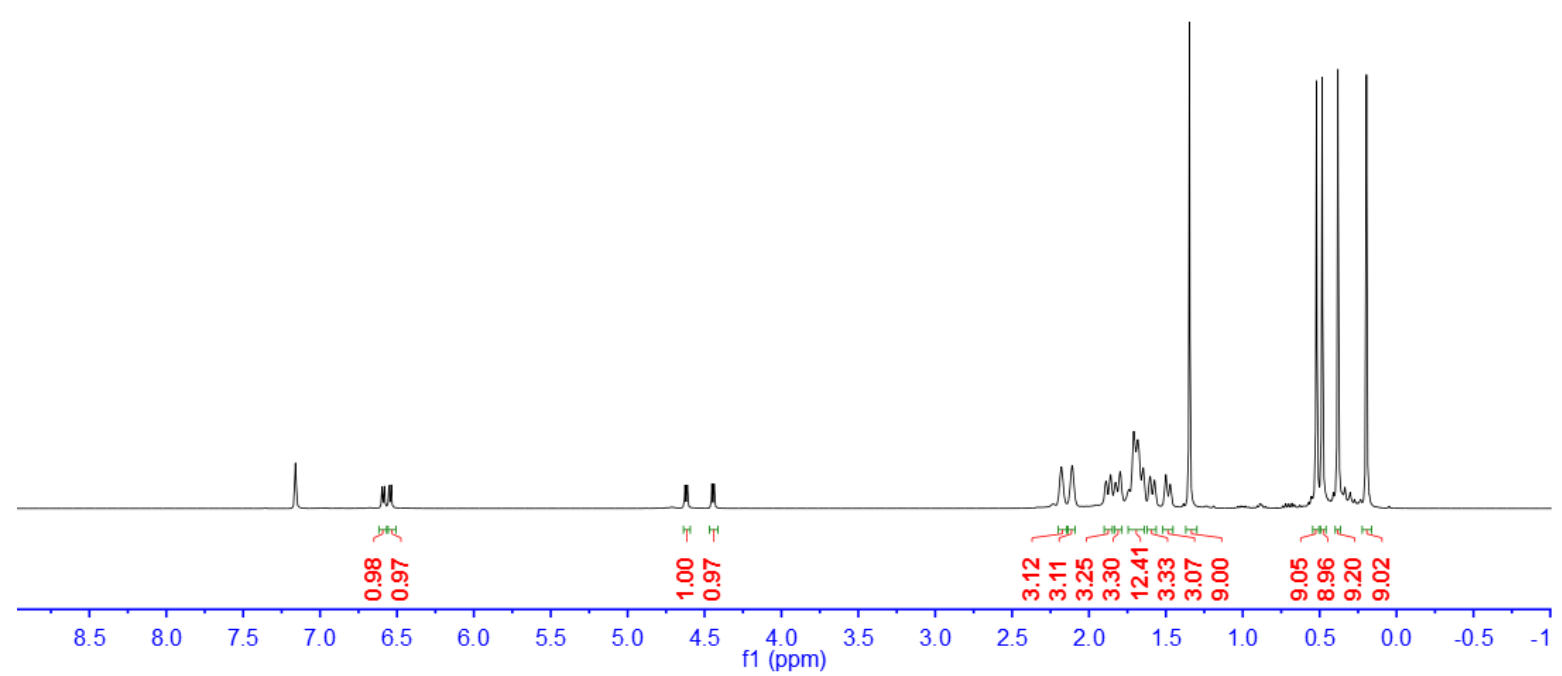

Figure S6. ${ }^{1} \mathrm{H}$ NMR spectrum of 3 in $\mathrm{C}_{6} \mathrm{D}_{6}$. 


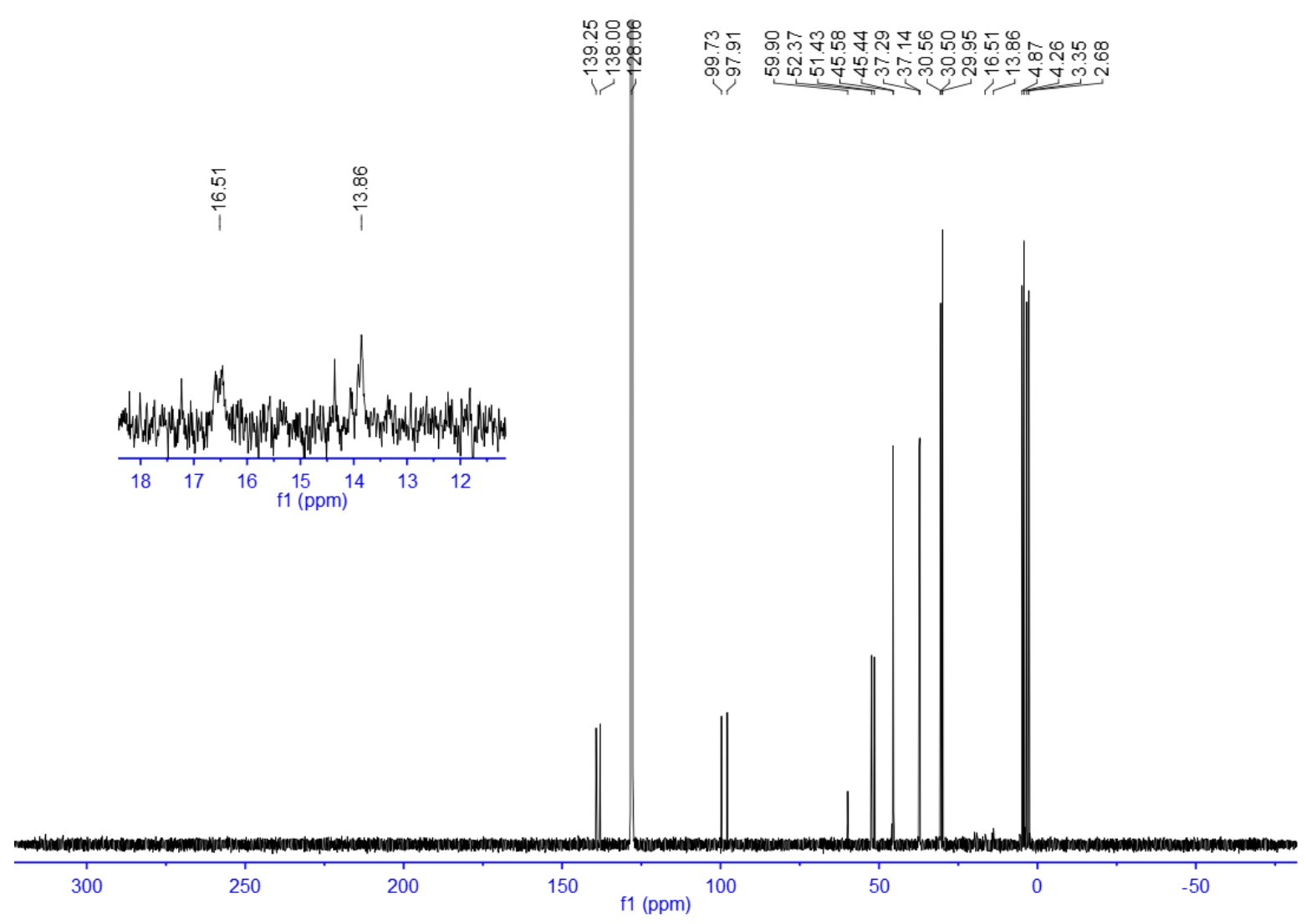

Figure S7. ${ }^{13} \mathrm{C}\left\{{ }^{1} \mathrm{H}\right\}$ NMR spectrum of 3 in $\mathrm{C}_{6} \mathrm{D}_{6}$.

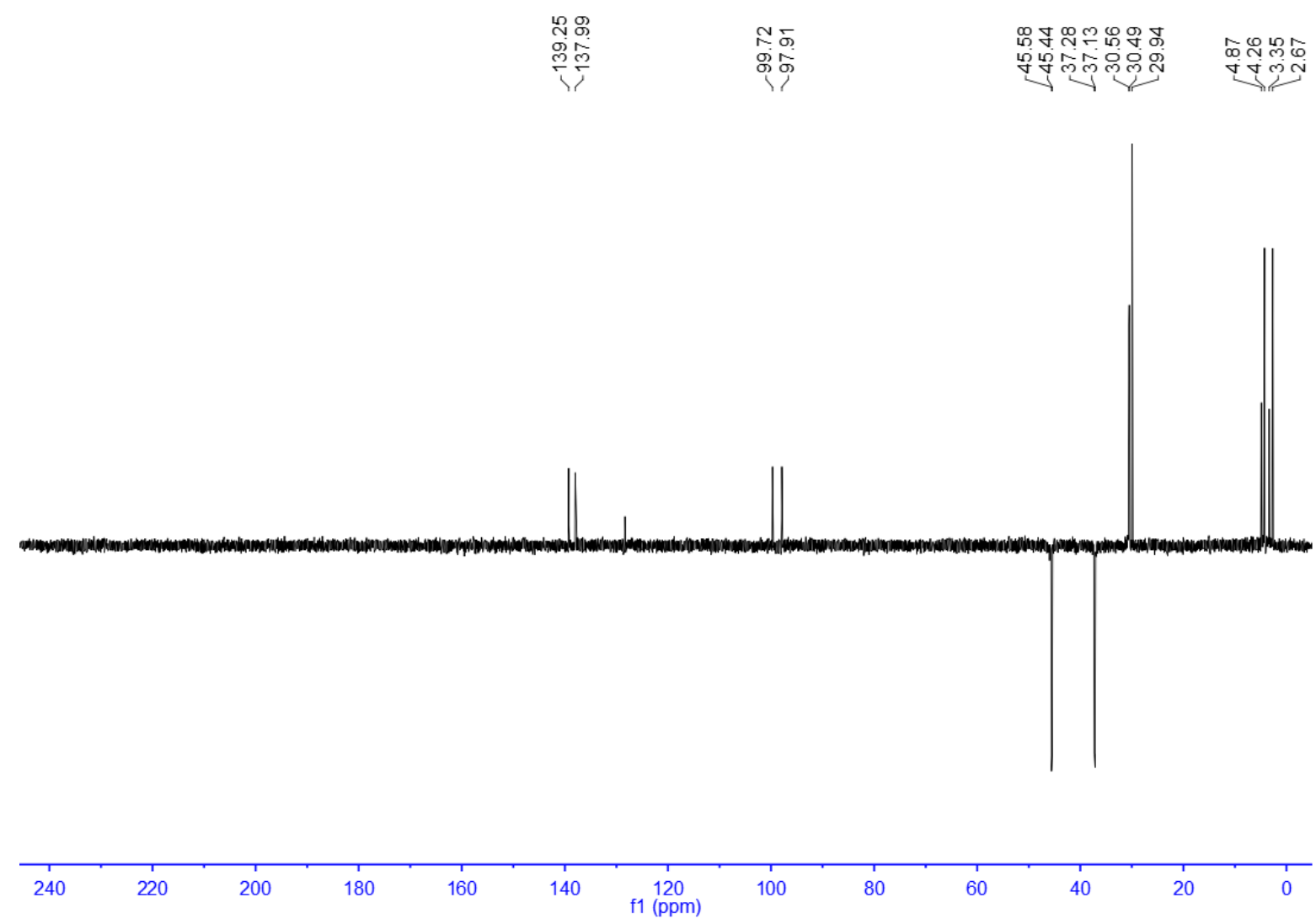

Figure S8. ${ }^{13} \mathrm{C}$ (DEPT 135) NMR spectrum of 3 in $\mathrm{C}_{6} \mathrm{D}_{6}$. 


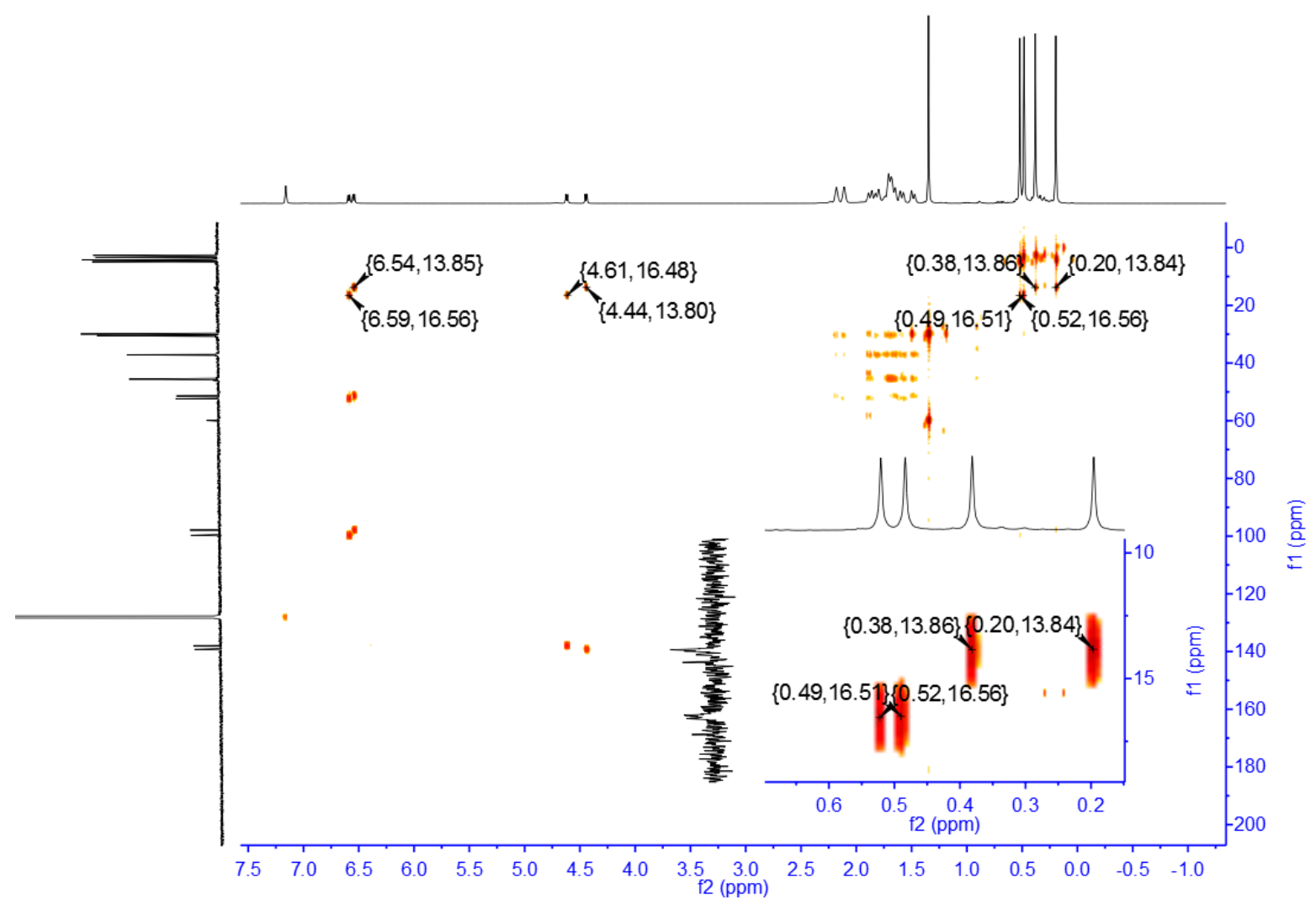

Figure S9. ${ }^{1} \mathrm{H}-{ }^{13} \mathrm{C}$ HMBC NMR spectrum of 3 in $\mathrm{C}_{6} \mathrm{D}_{6}$.

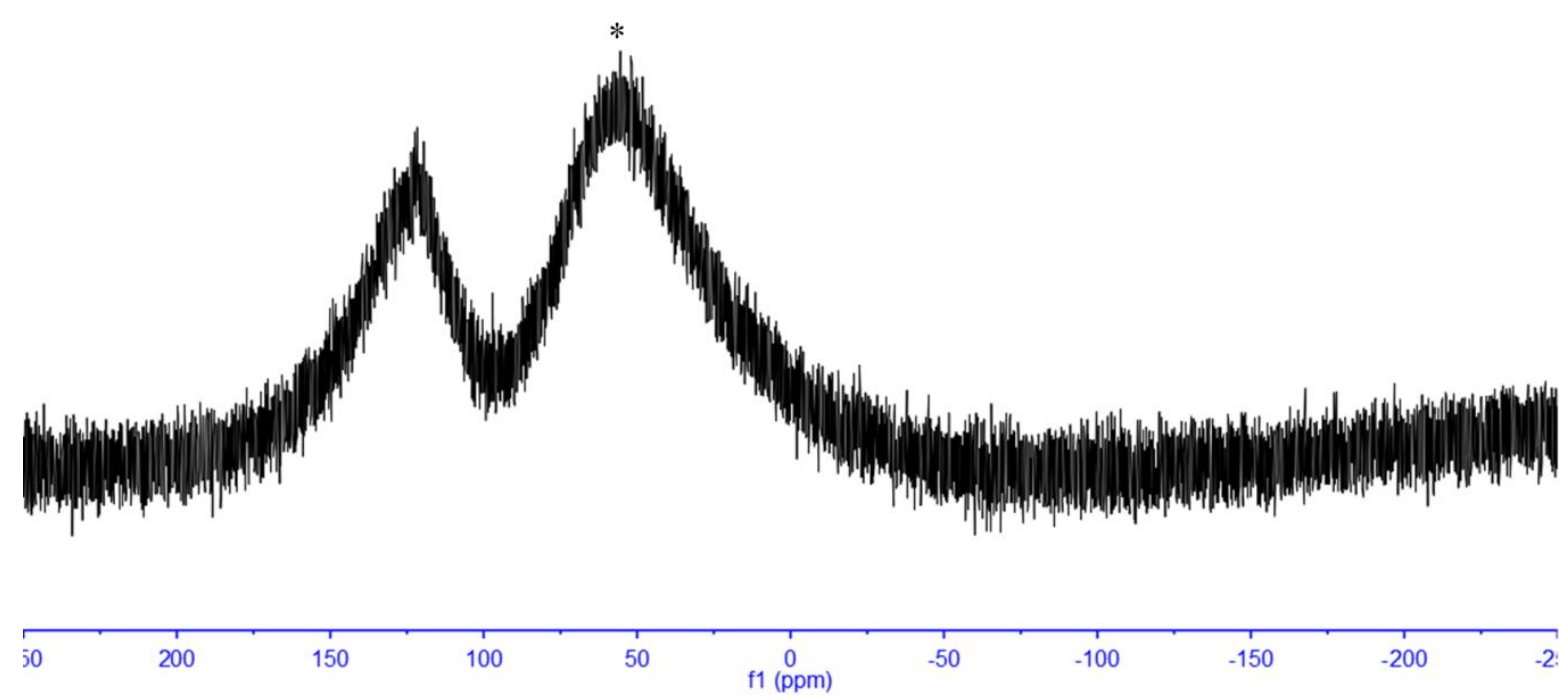

Figure S10. ${ }^{27} \mathrm{Al}\left\{{ }^{1} \mathrm{H}\right\}$ NMR spectrum of $\mathbf{3}$ in $\mathrm{C}_{6} \mathrm{D}_{6}$. 

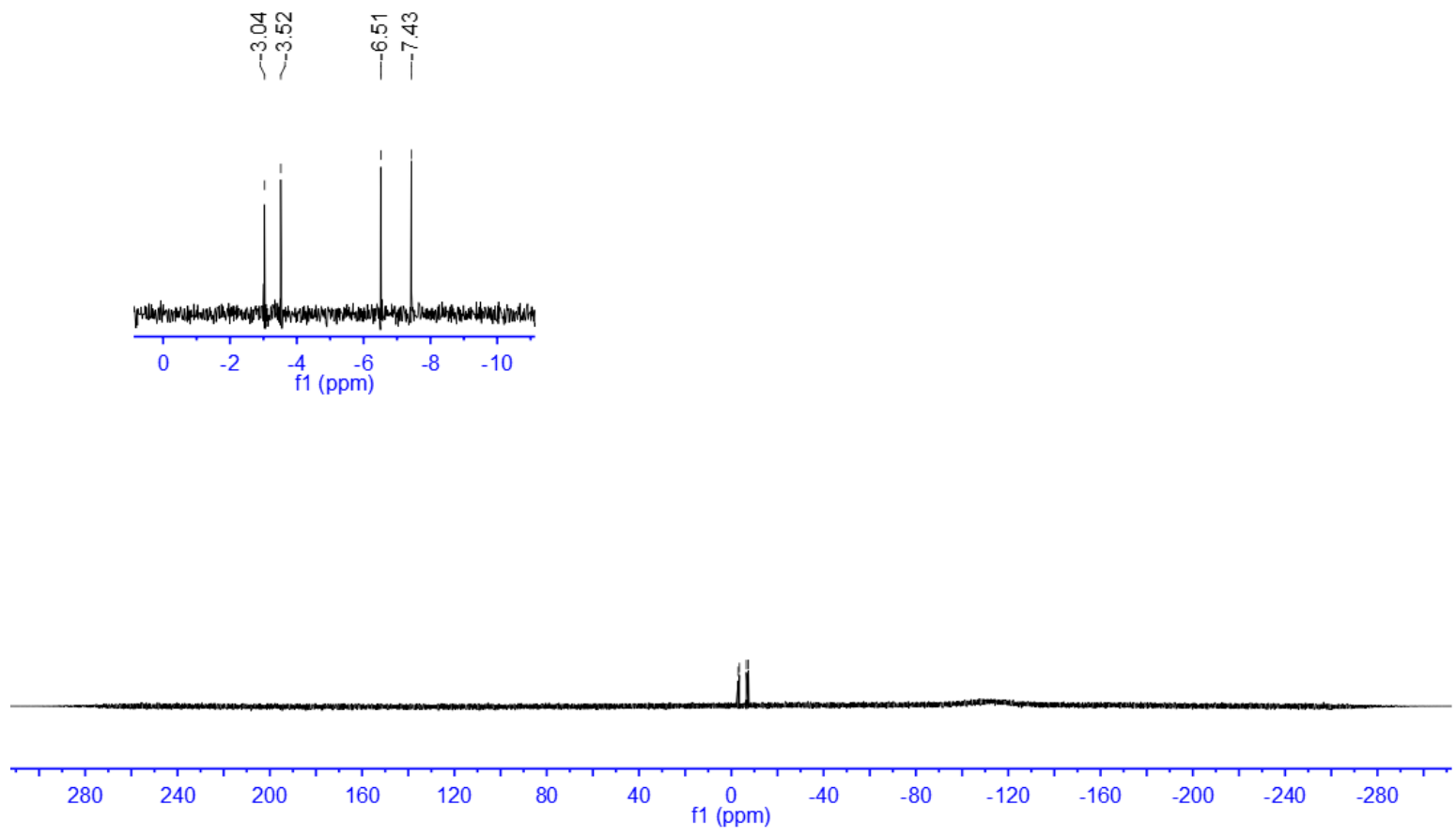

Figure $\mathrm{S} 11 .{ }^{29} \mathrm{Si}\left\{{ }^{1} \mathrm{H}\right\}$ NMR spectrum of 3 in $\mathrm{C}_{6} \mathrm{D}_{6}$.

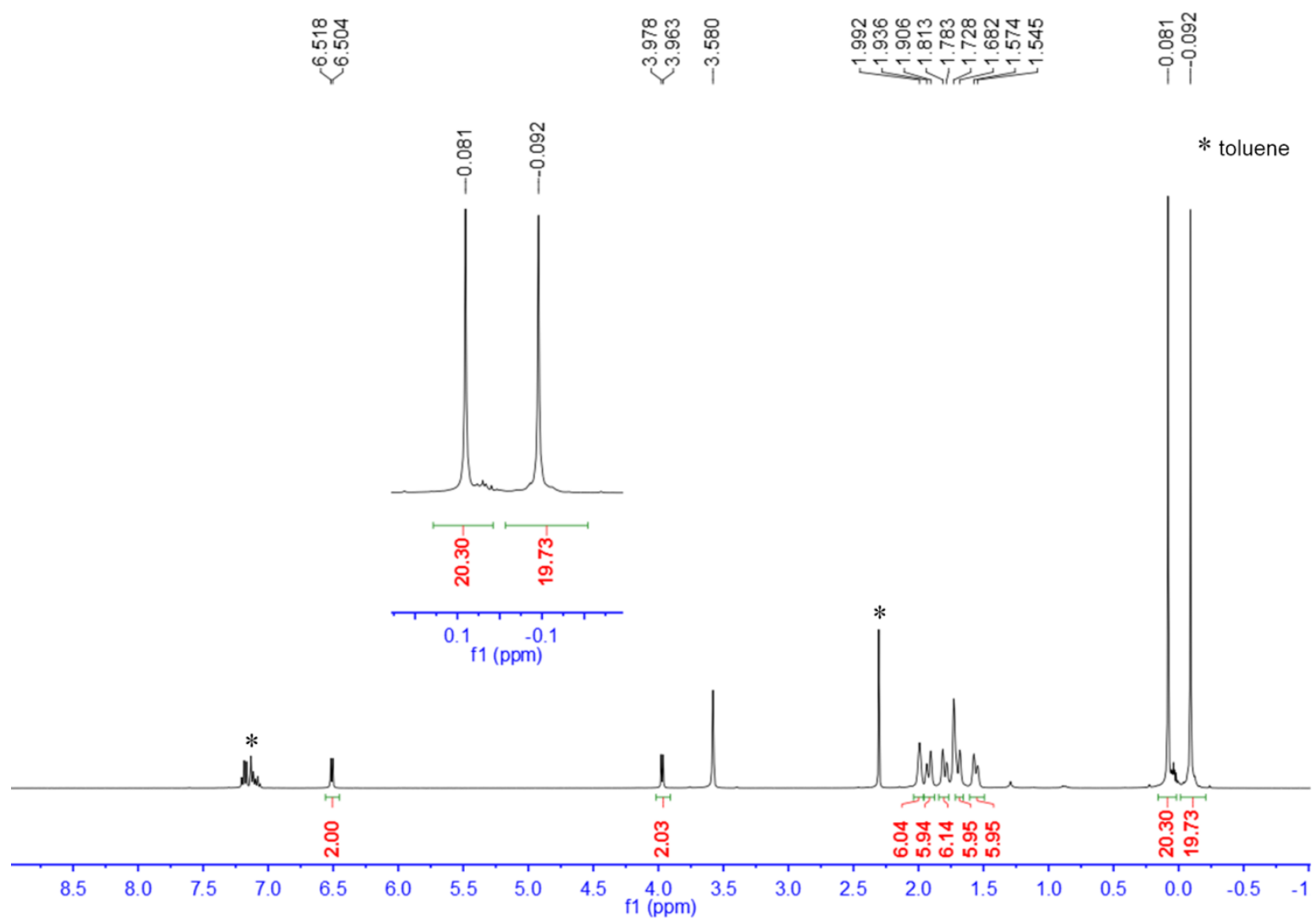

Figure S12. ${ }^{1} \mathrm{H}$ NMR spectrum of 4 in THF-d8. 

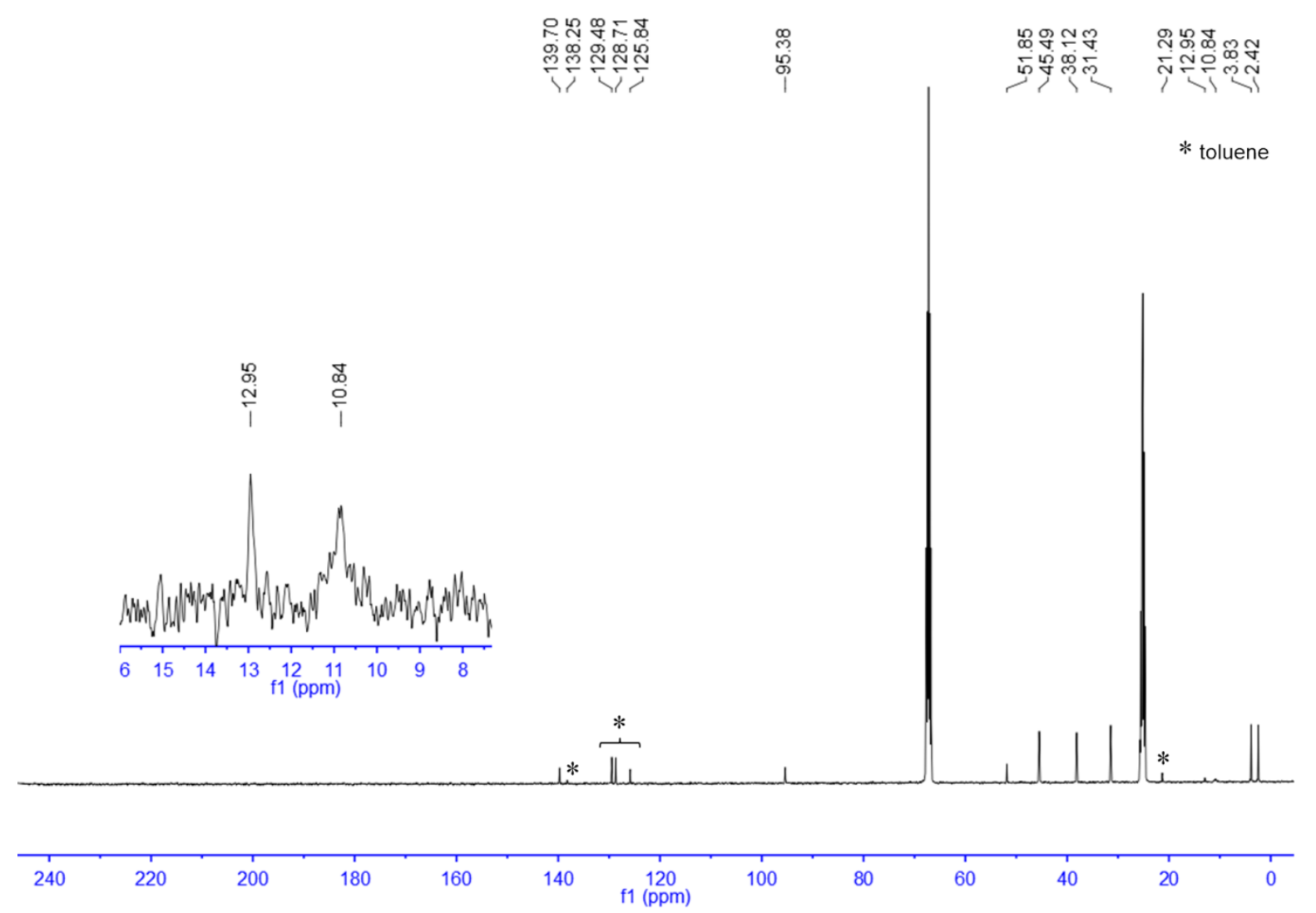

Figure S13. ${ }^{13} \mathrm{C}\left\{{ }^{1} \mathrm{H}\right\}$ NMR spectrum of 4 in THF- $\mathrm{d}_{8}$.

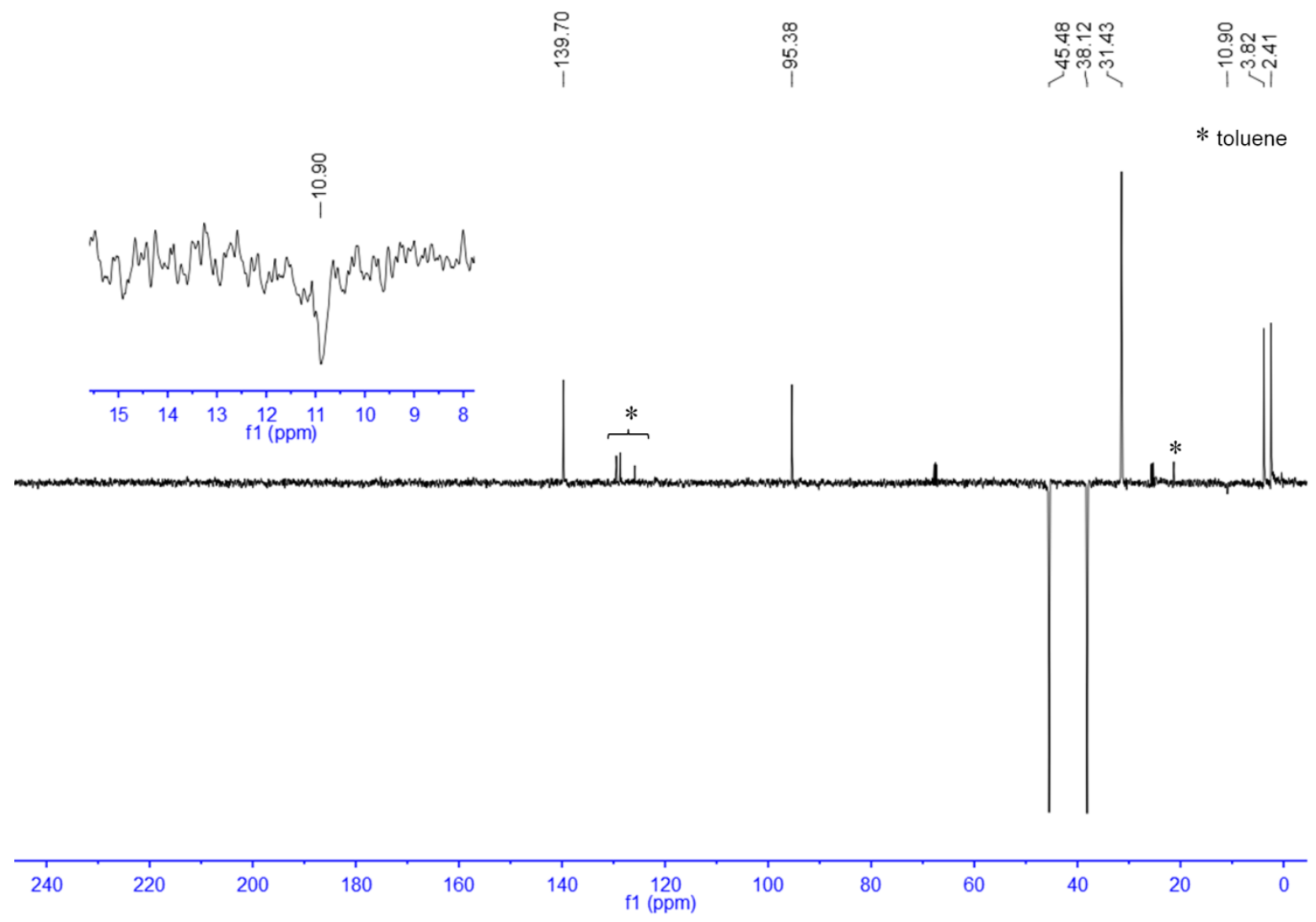

Figure S14. ${ }^{13} \mathrm{C}$ (DEPT 135) NMR spectrum of 4 in THF-d8. 


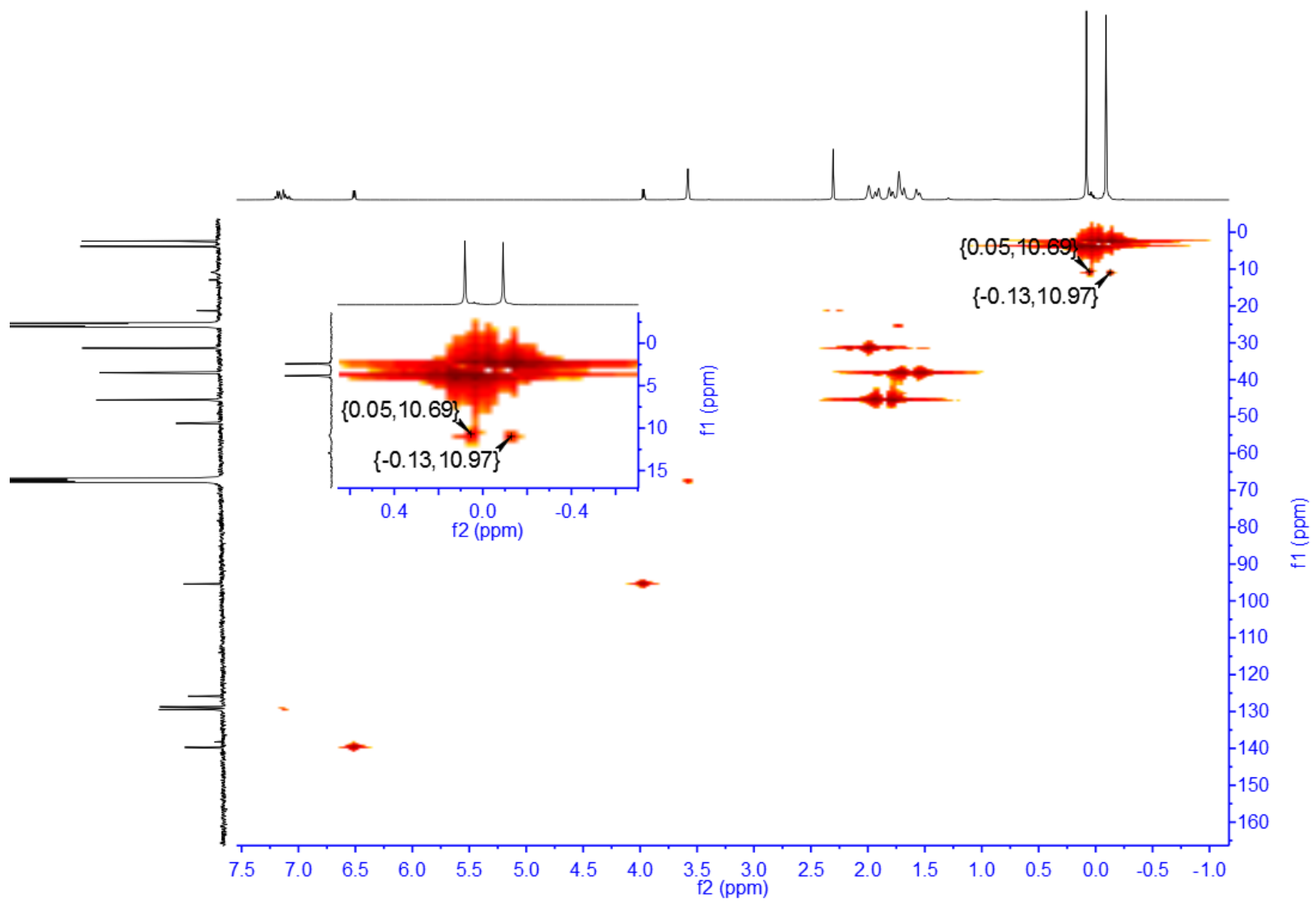

Figure S15. ${ }^{1} \mathrm{H}-{ }^{13} \mathrm{C}$ HMQC NMR spectrum of 4 in THF-d8.

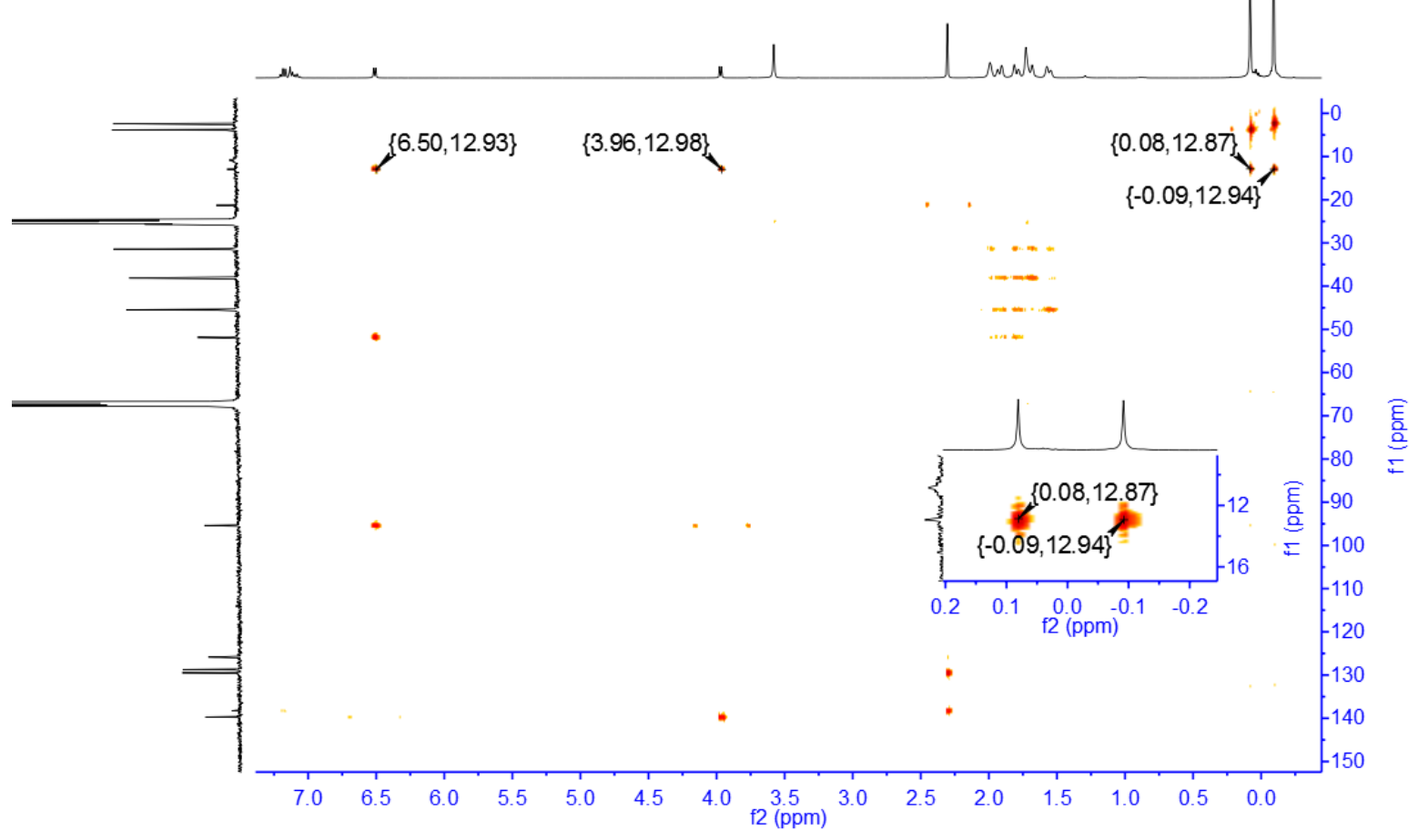

Figure S16. ${ }^{1} \mathrm{H}^{-13} \mathrm{C}$ HMBC NMR spectrum of 4 in THF-d8.

S19 


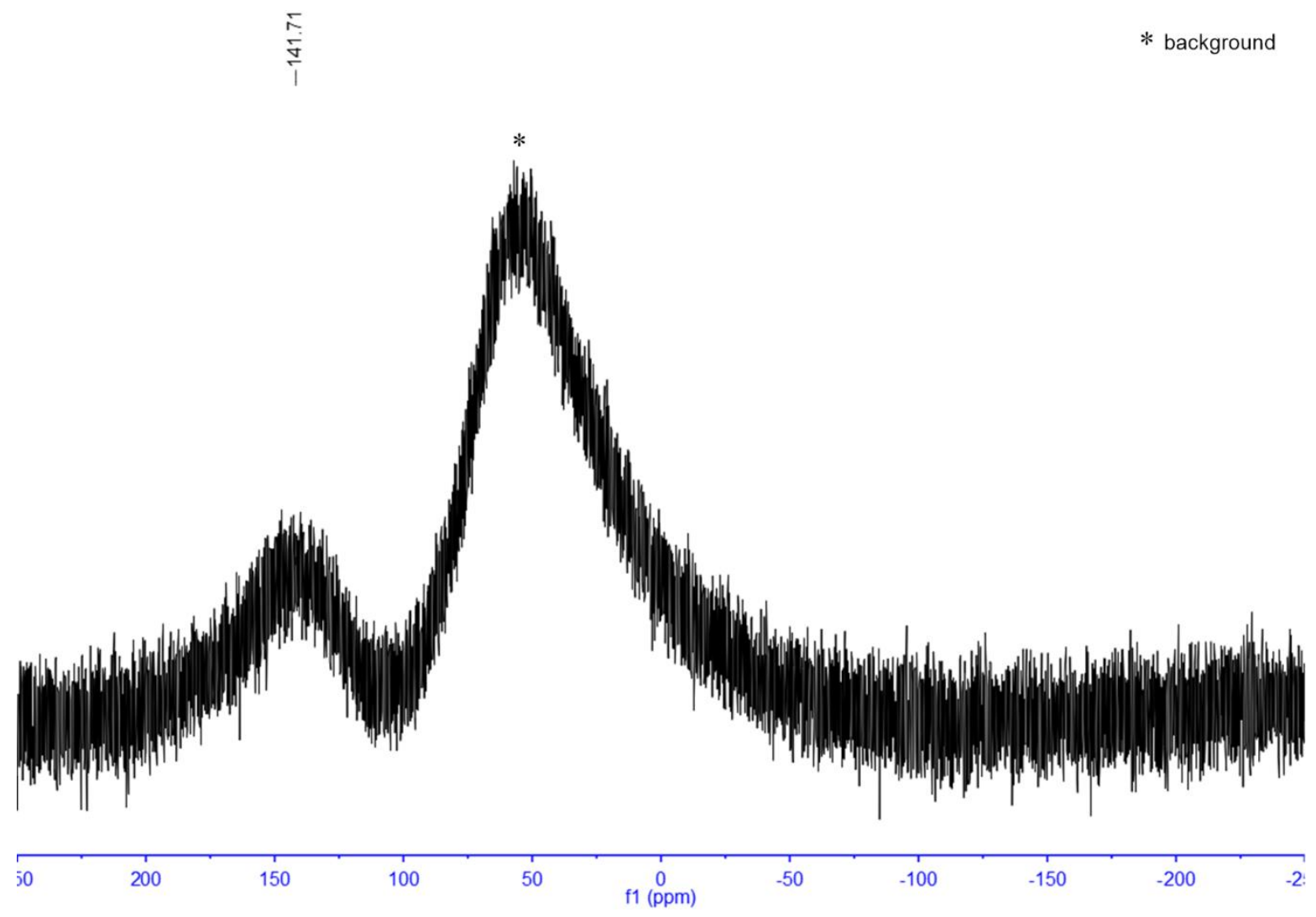

Figure S17. ${ }^{27} \mathrm{Al}\left\{{ }^{1} \mathrm{H}\right\}$ NMR spectrum of 4 in THF-d8.
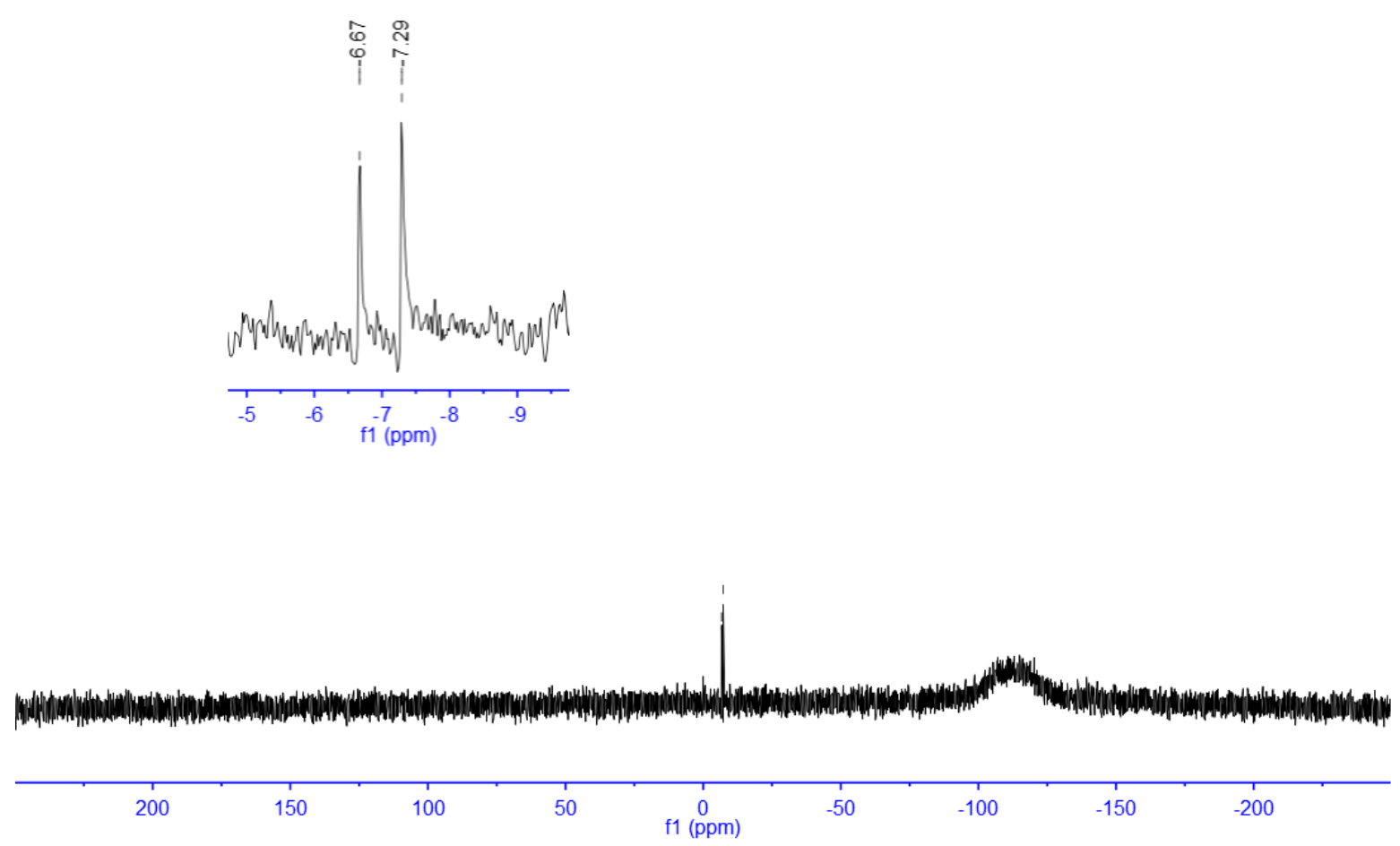

Figure S18. ${ }^{29} \mathrm{Si}\left\{{ }^{1} \mathrm{H}\right\}$ NMR spectrum of 4 in THF-d8. 


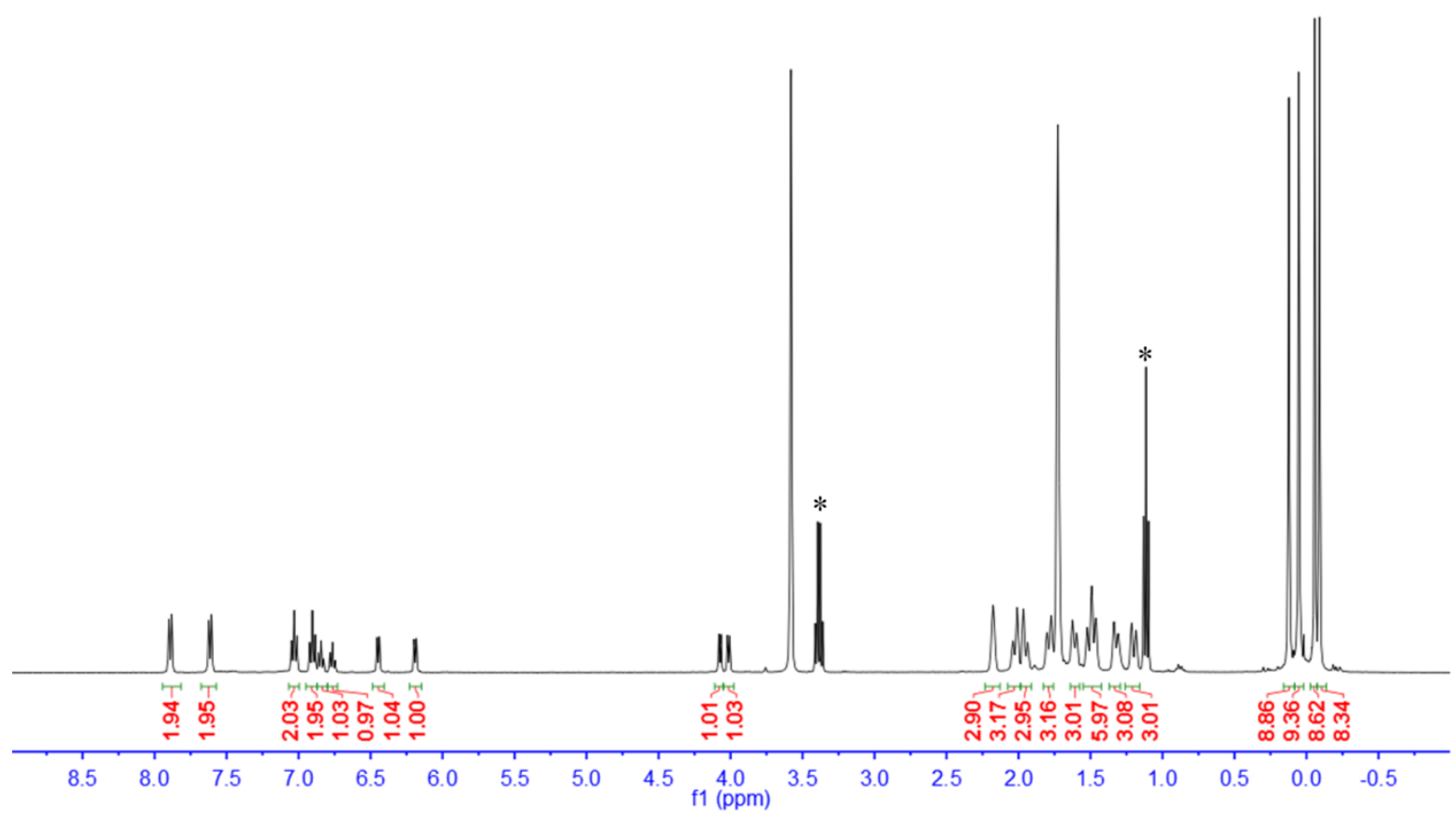

Figure S19. ${ }^{1} \mathrm{H}$ NMR spectrum of 5 in THF-d8.

$* \mathrm{Et}_{2} \mathrm{O}$

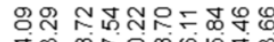

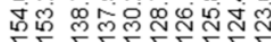

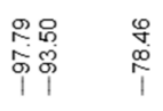

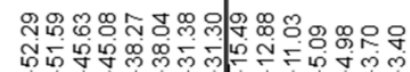

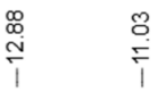

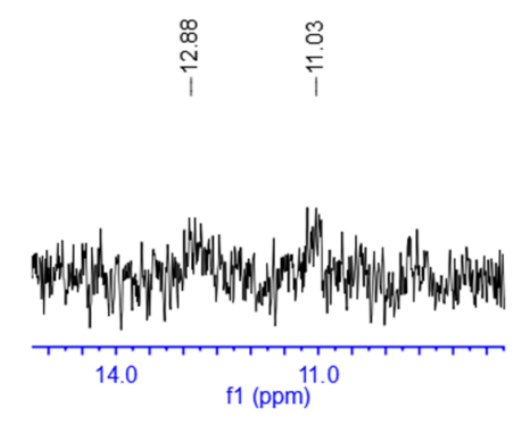

1 (ppm)

240

$220 \quad 200$

180

160

140

120

100

80

60

Figure S20. ${ }^{13} \mathrm{C}\left\{{ }^{1} \mathrm{H}\right\}$ NMR spectrum of 5 in THF-d8. 


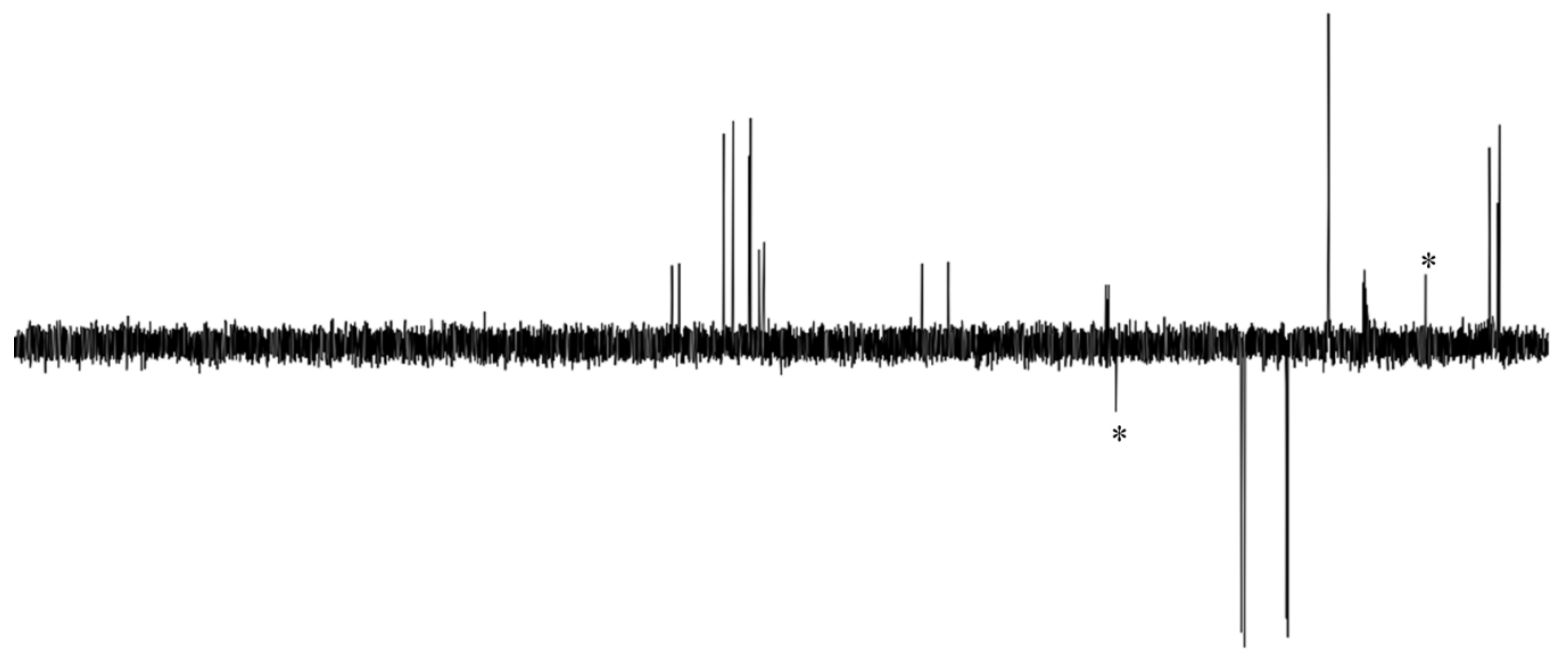

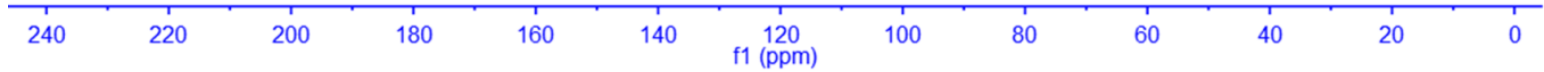

Figure S21. ${ }^{13} \mathrm{C}(\mathrm{DEPT} 135)$ NMR spectrum of 5 in THF-d8.

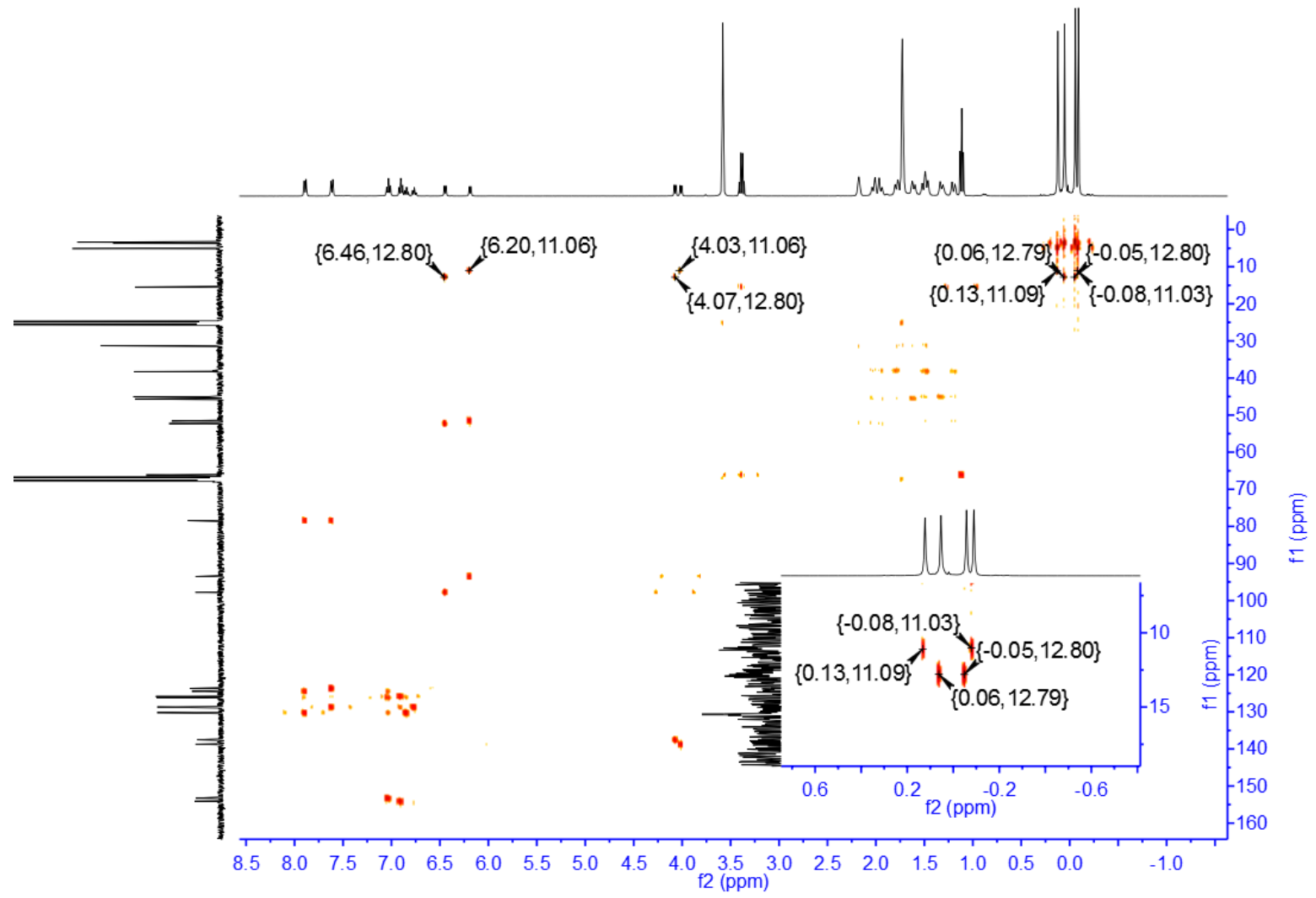

Figure S22. ${ }^{1} \mathrm{H}-{ }^{13} \mathrm{C}$ HMBC NMR spectrum of 5 in THF-d8. 

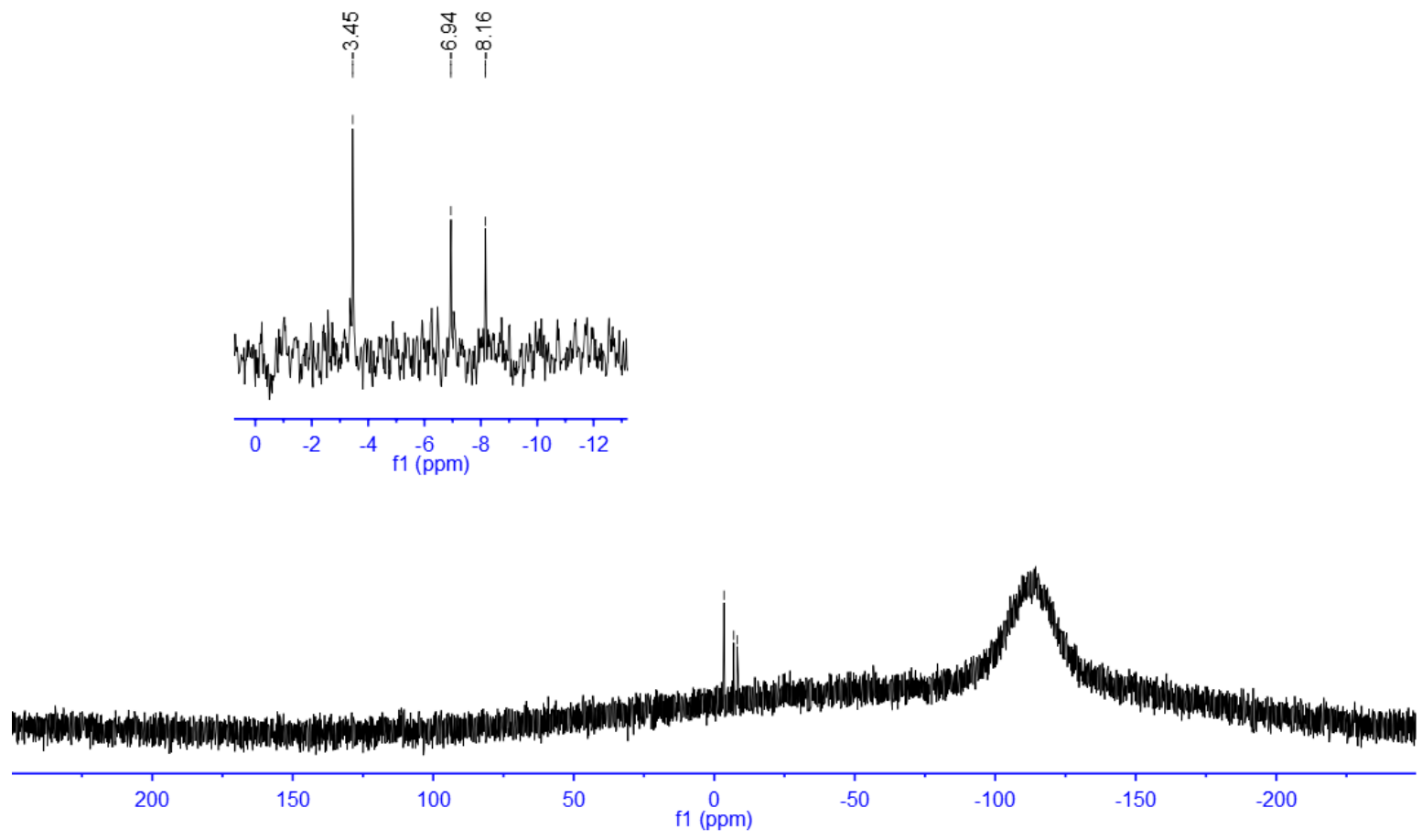

Figure S23. ${ }^{29} \mathrm{Si}\left\{{ }^{1} \mathrm{H}\right\}$ NMR spectrum of $\mathbf{5}$ in THF-d8.

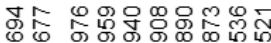

(2)

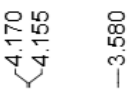

象

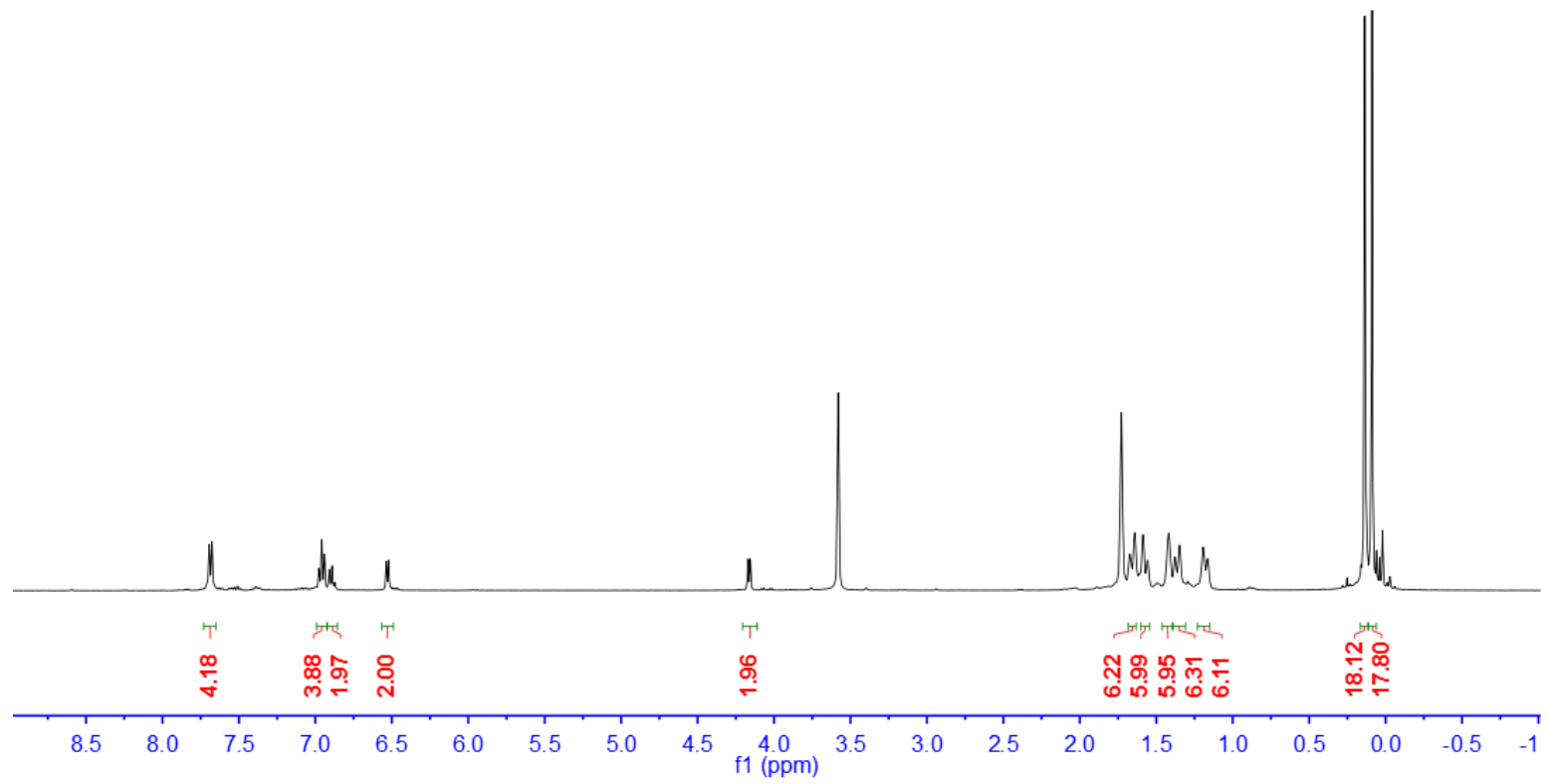

Figure S24. ${ }^{1} \mathrm{H}$ NMR spectrum of 6 in THF-d8. 


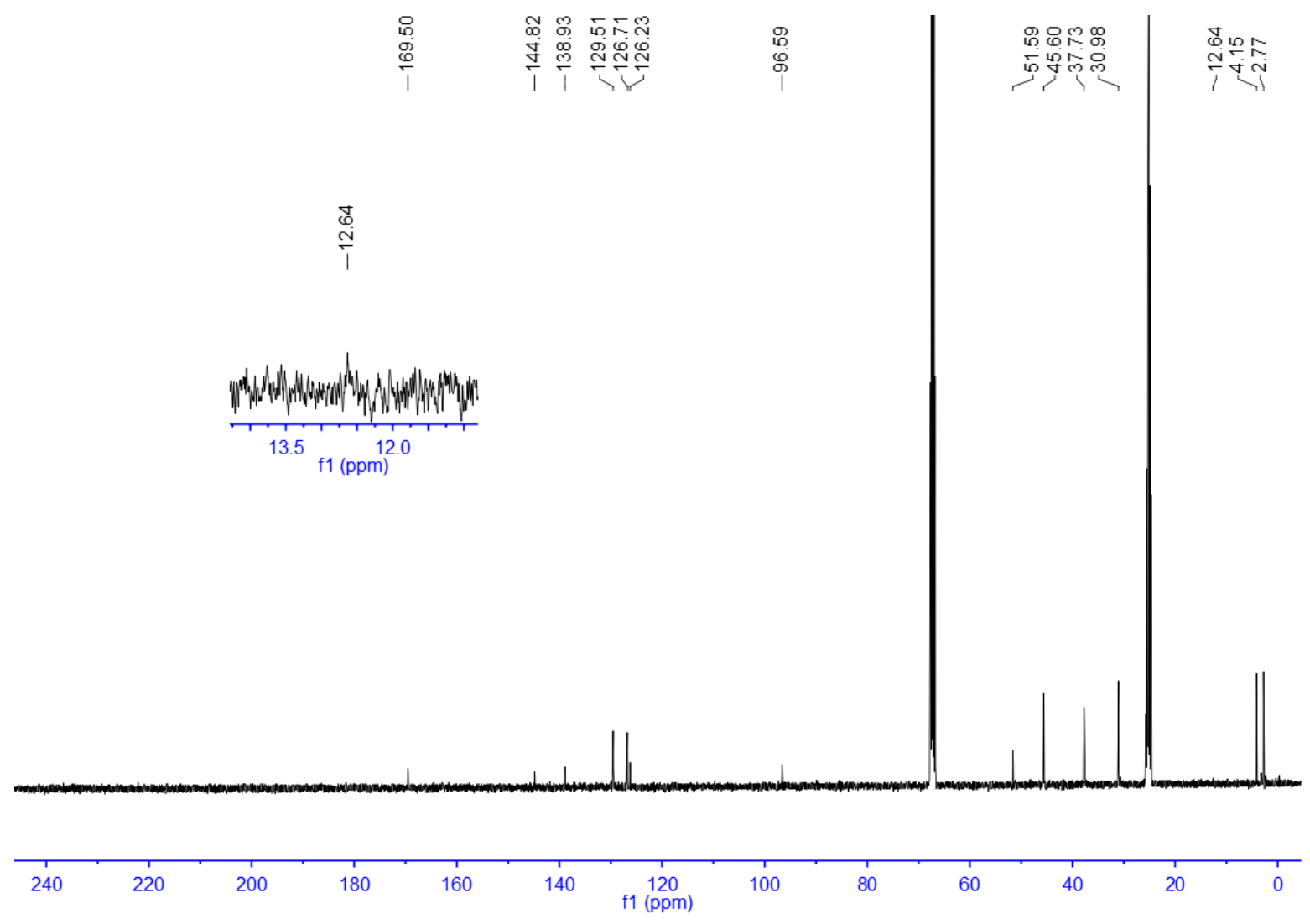

Figure S25. ${ }^{13} \mathrm{C}\left\{{ }^{1} \mathrm{H}\right\}$ NMR spectrum of 6 in THF-d8.
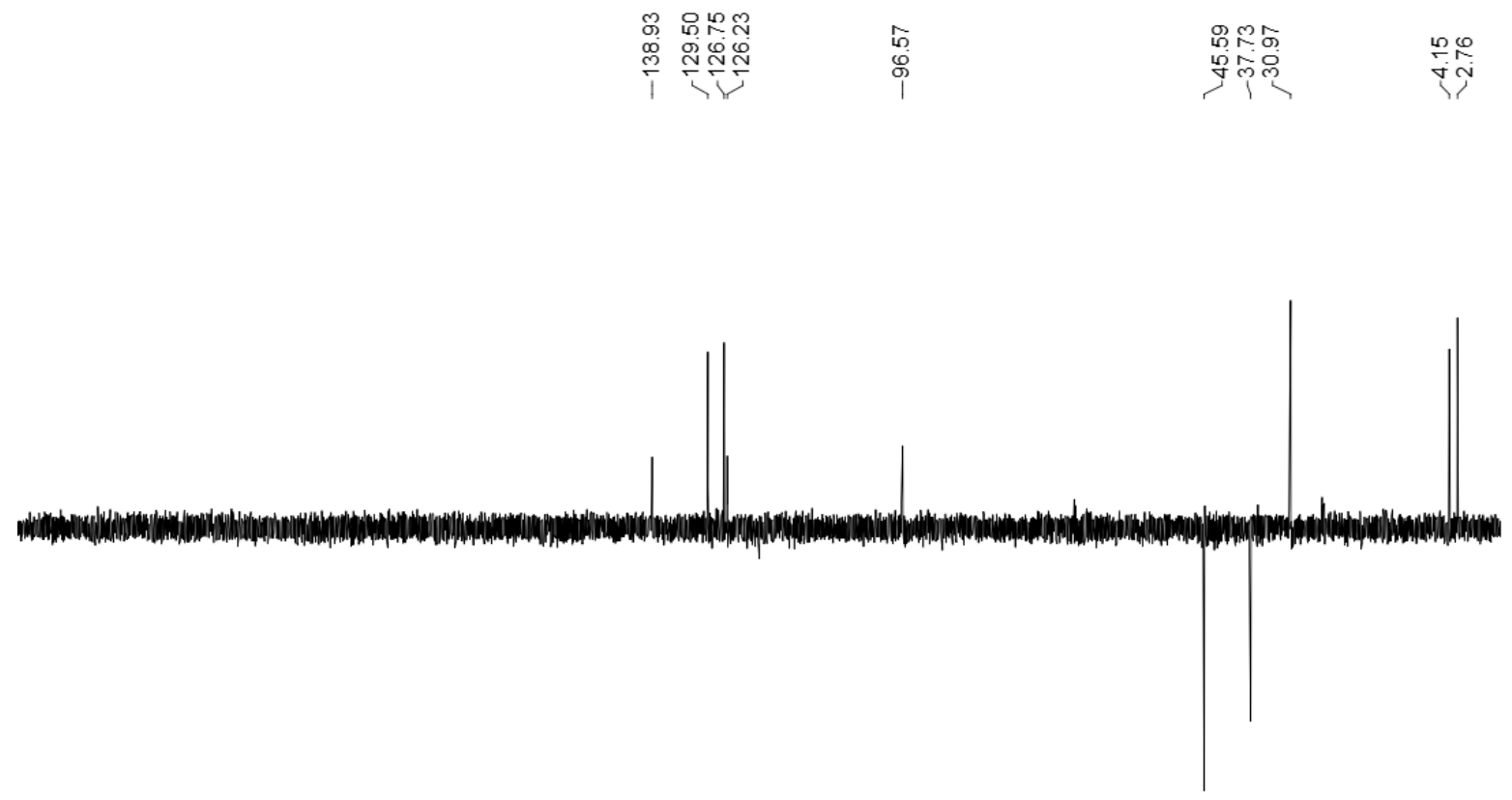

\begin{tabular}{|c|c|c|c|c|c|c|c|c|c|c|c|}
\hline 240 & 220 & 200 & 180 & 160 & 140 & $\begin{array}{c}120 \\
\mathrm{f} 1(\mathrm{ppm})\end{array}$ & 100 & 80 & 60 & 40 & 20 \\
\hline
\end{tabular}

Figure S26. ${ }^{13} \mathrm{C}$ (DEPT 135) NMR spectrum of 6 in THF-d8. 


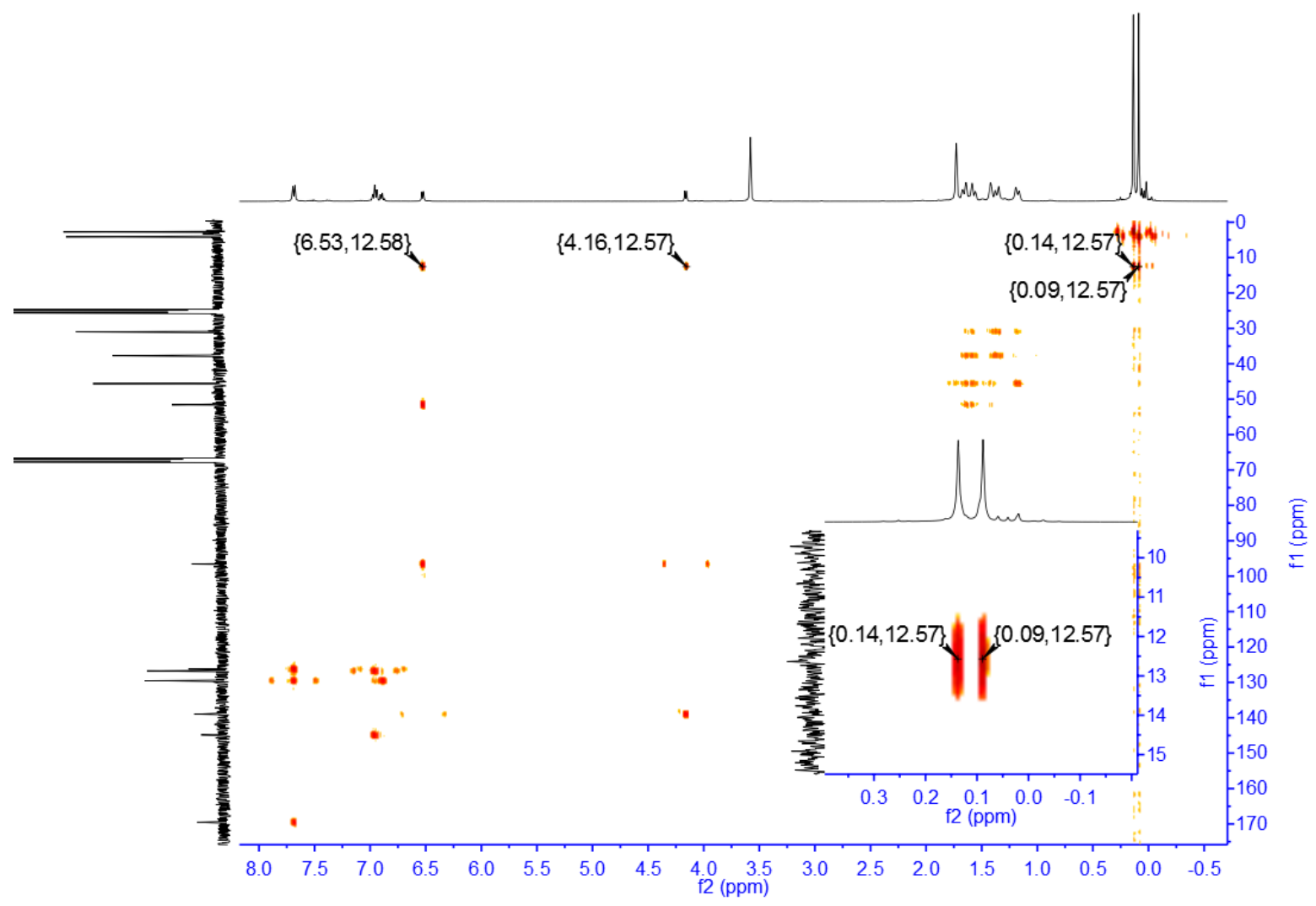

Figure S27. ${ }^{1} \mathrm{H}-{ }^{13} \mathrm{C}$ HMBC NMR spectrum of 6 in THF-d8.

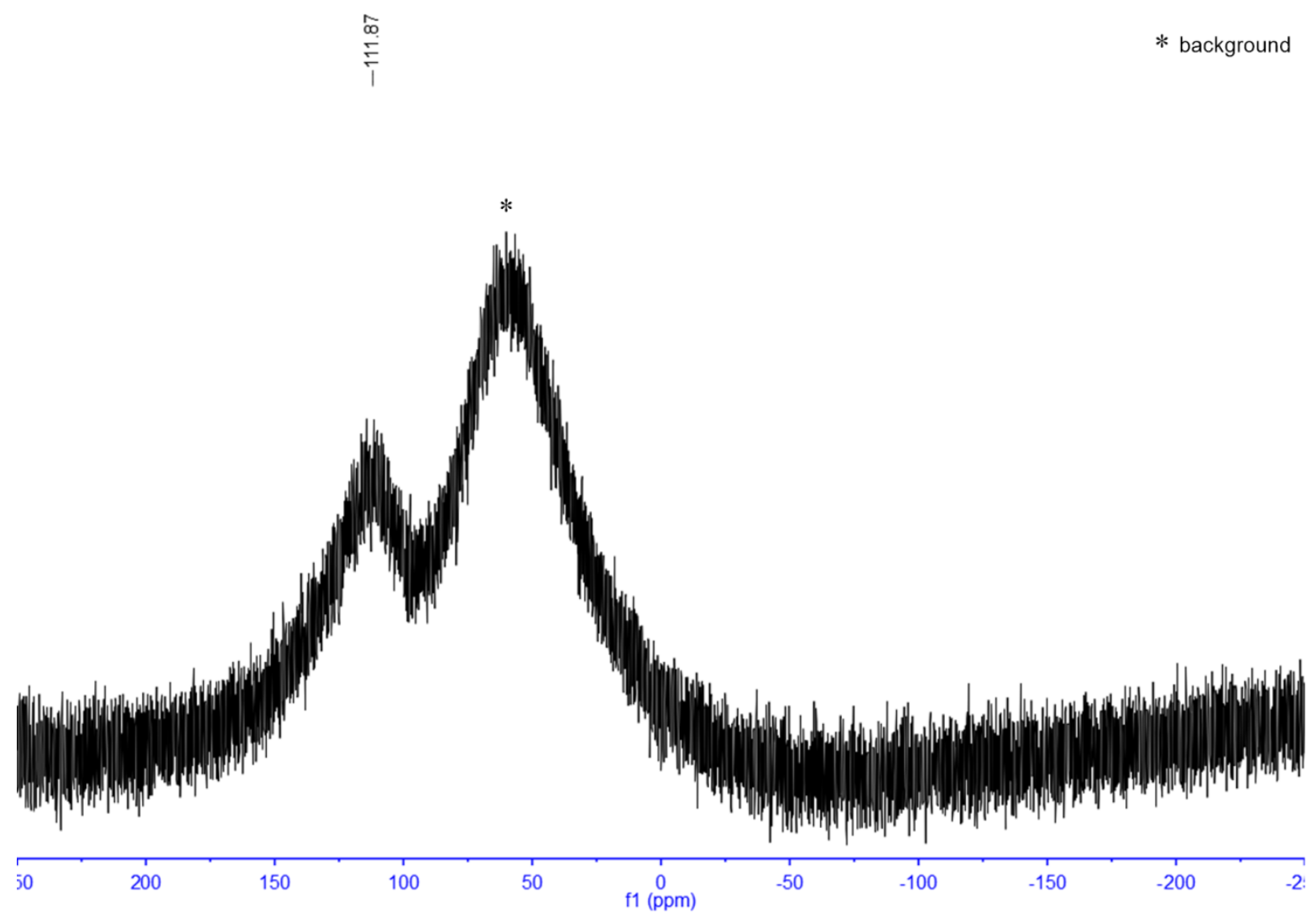

Figure S28. ${ }^{27} \mathrm{AI}\left\{{ }^{1} \mathrm{H}\right\}$ NMR spectrum of 6 in THF-d8. 


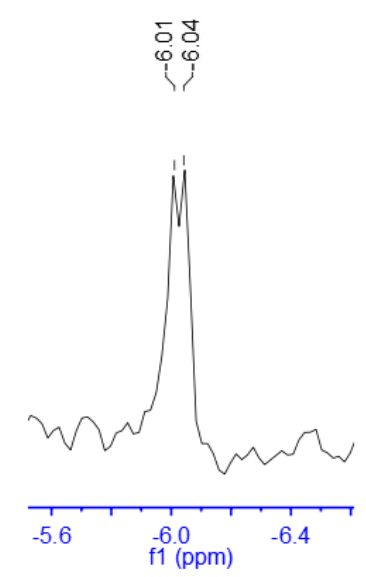

$200 \quad 150$

100

50

f1 $\stackrel{0}{\text { ppm) }}$

$-50$

$-100$

$-150$

$-200$

Figure S29. ${ }^{29} \mathrm{Si}\left\{{ }^{1} \mathrm{H}\right\}$ NMR spectrum of 6 in THF-d8.

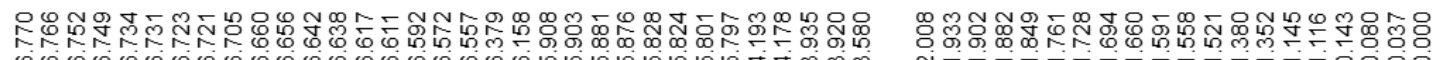
ị

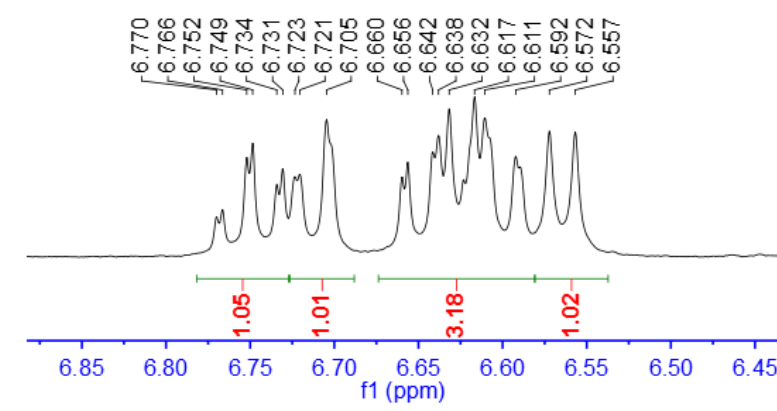

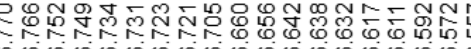




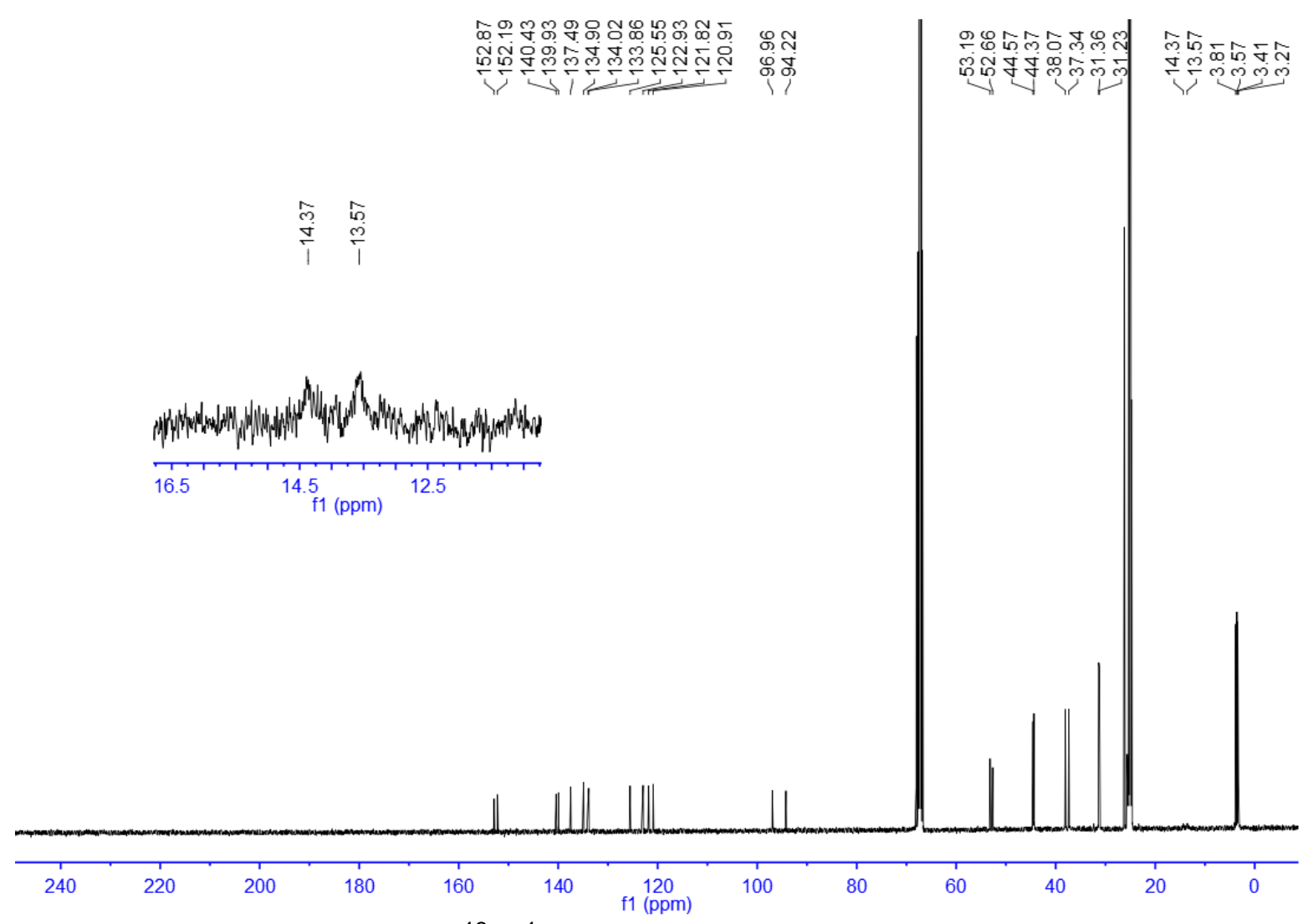

Figure S31. ${ }^{13} \mathrm{C}\left\{{ }^{1} \mathrm{H}\right\}$ NMR spectrum of 7 in THF-d8.

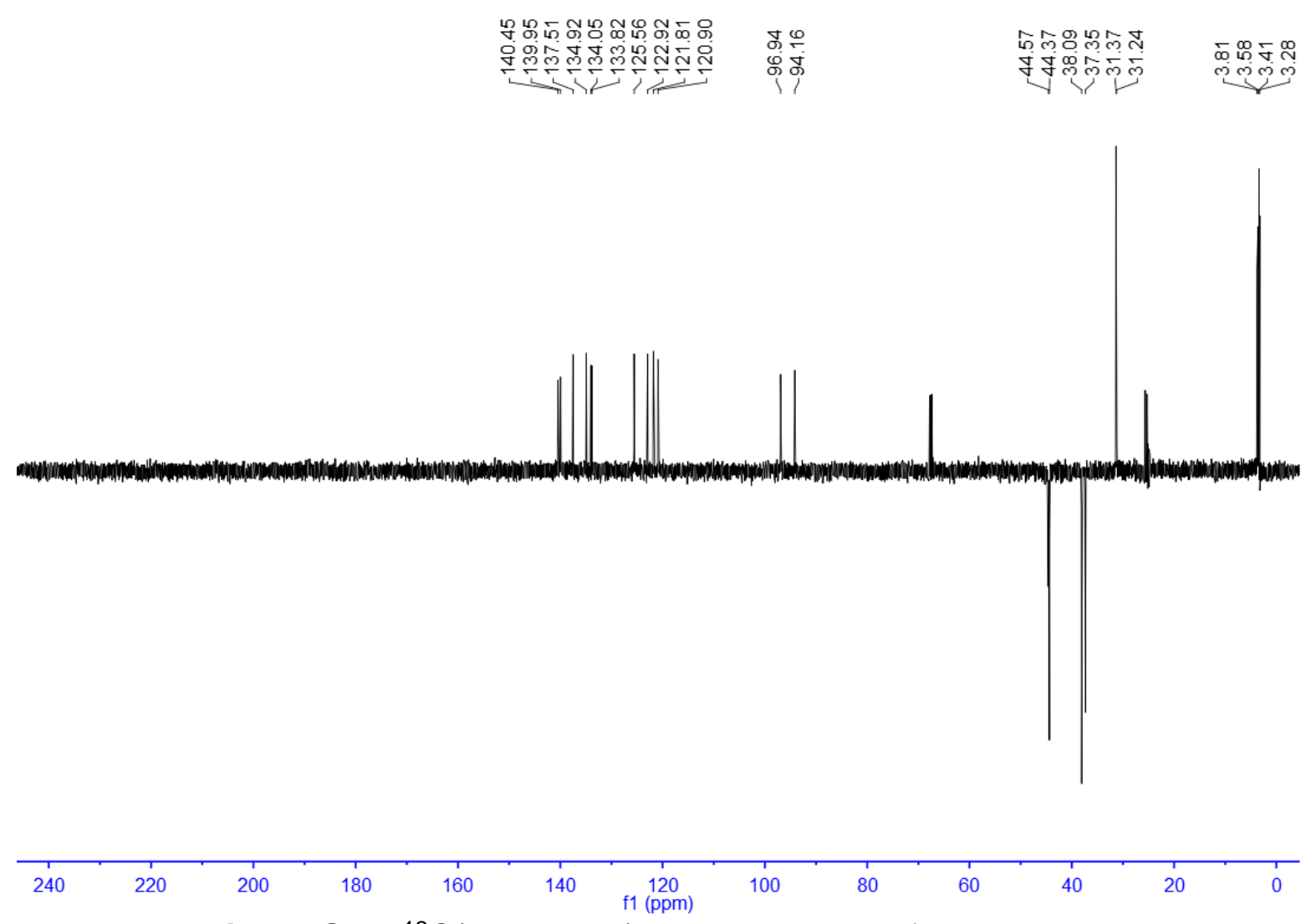

Figure S32. ${ }^{13} \mathrm{C}$ (DEPT 135) NMR spectrum of $\mathbf{7}$ in THF-d8. 


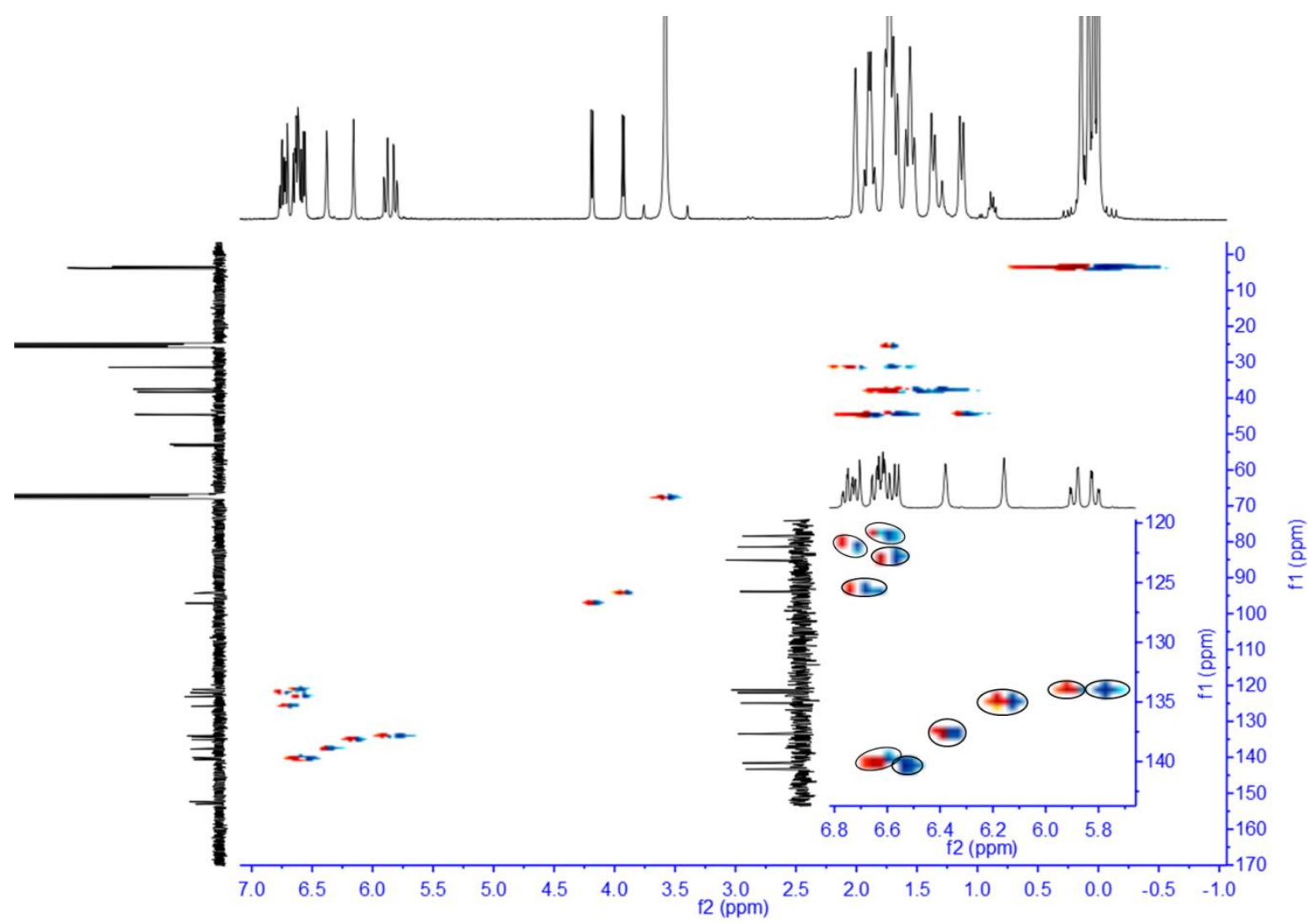

Figure S33. ${ }^{1} \mathrm{H}-{ }^{13} \mathrm{C}$ HSQC NMR spectrum of 7 in THF-d8.

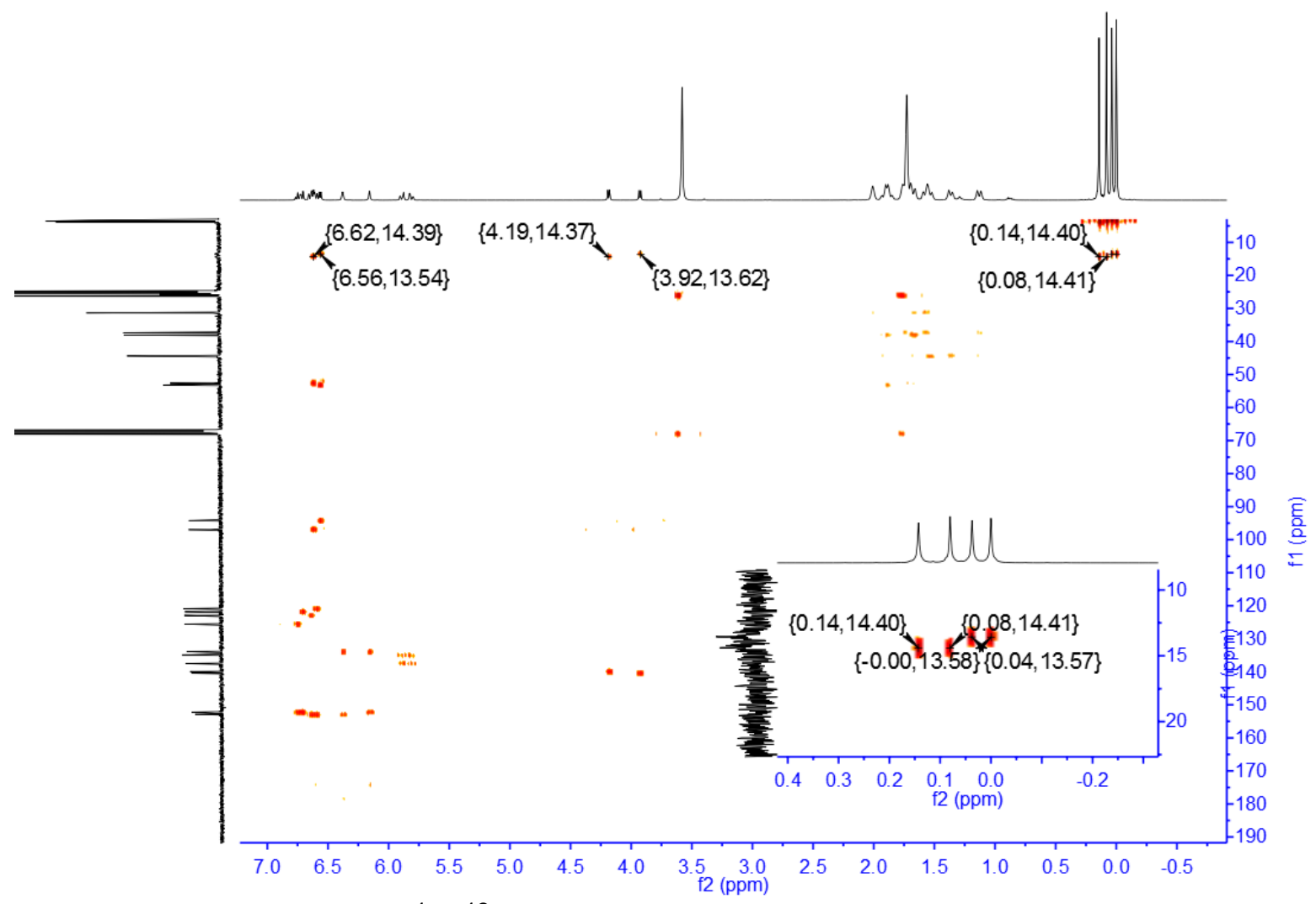

Figure S34. ${ }^{1} \mathrm{H}-{ }^{13} \mathrm{C}$ HMBC NMR spectrum of 7 in THF-d8. 


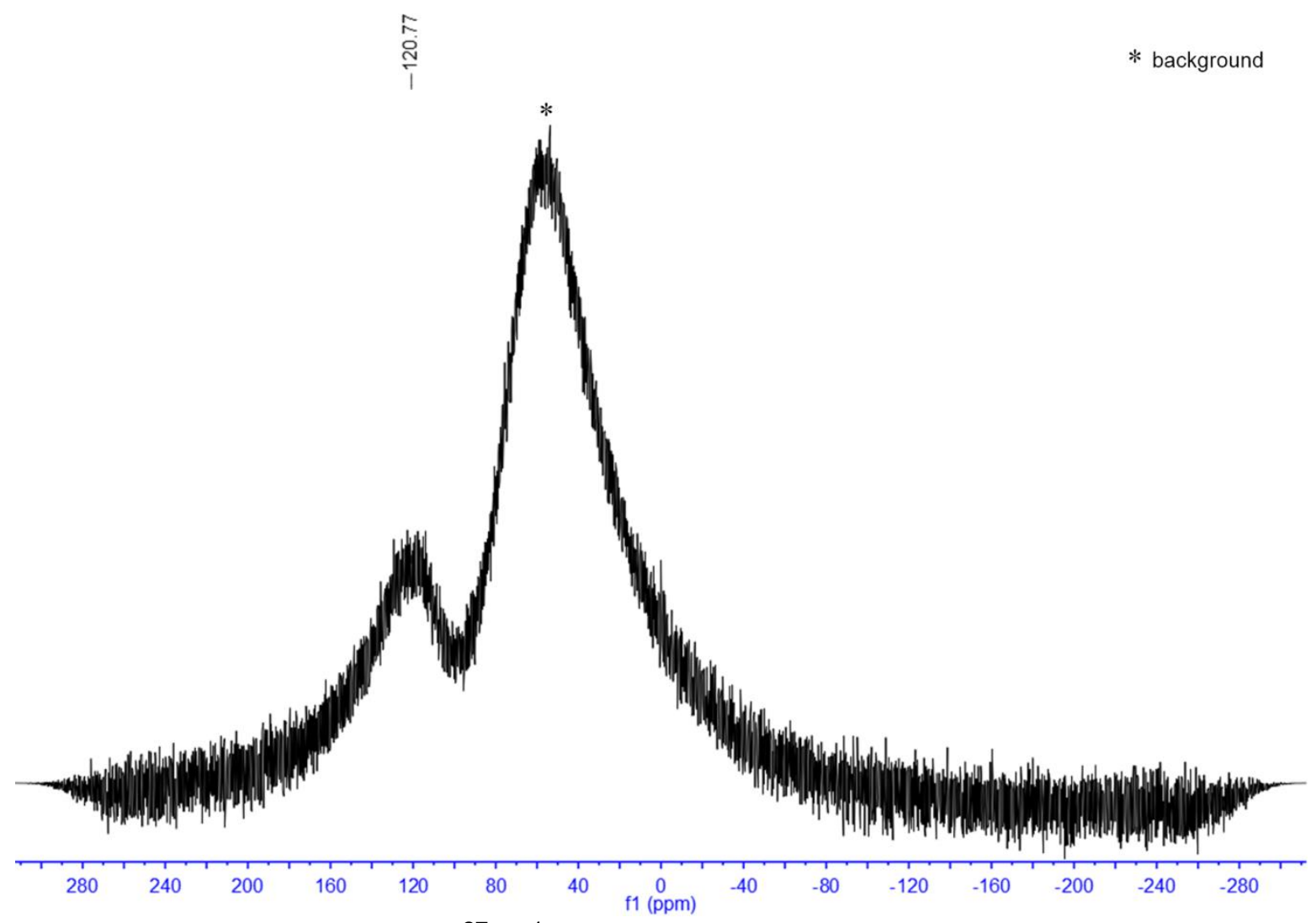

Figure S35. ${ }^{27} \mathrm{Al}\left\{{ }^{1} \mathrm{H}\right\}$ NMR spectrum of $7 \mathrm{in}$ THF-d8.

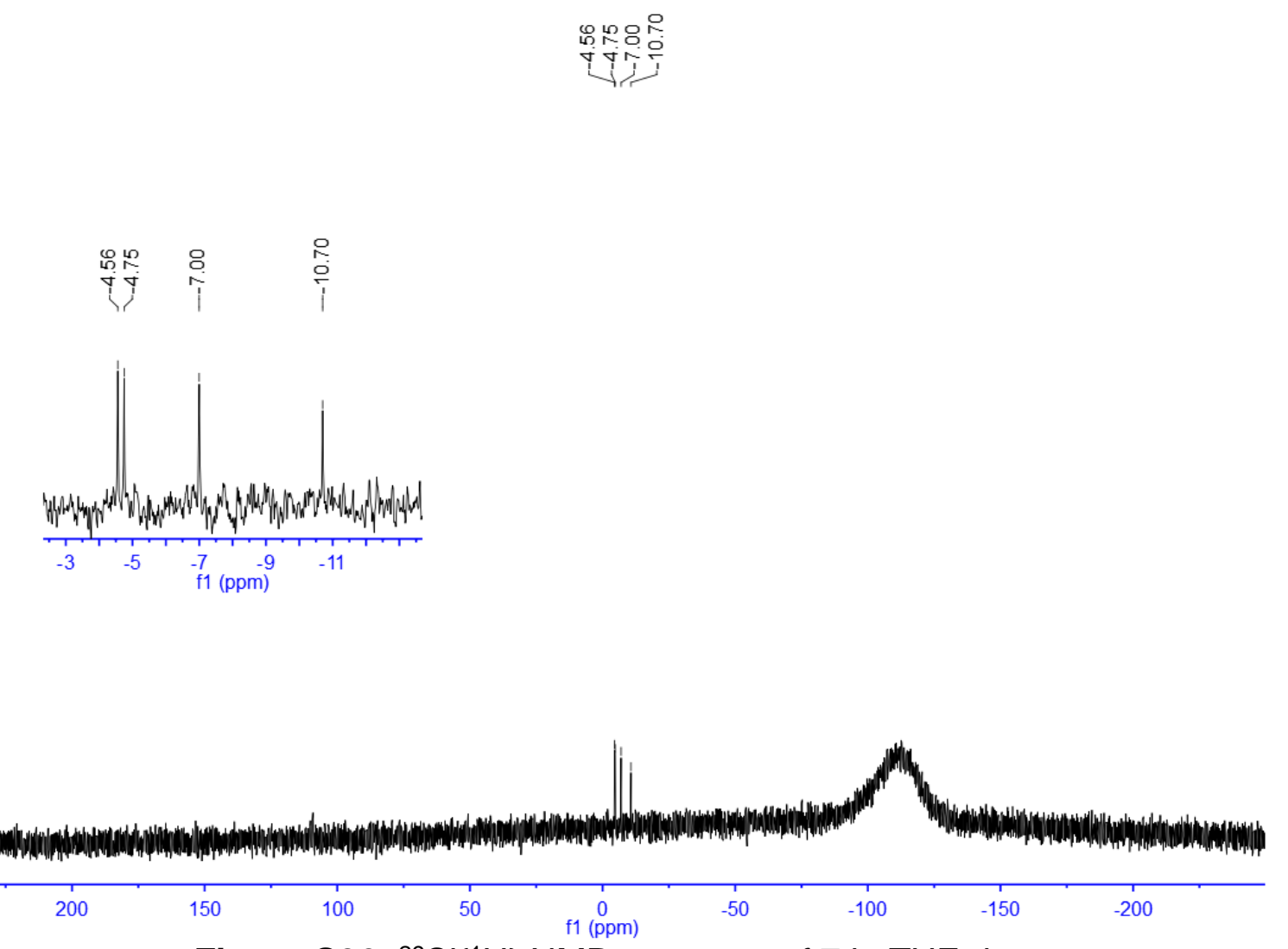

Figure S36. ${ }^{29} \mathrm{Si}\left\{{ }^{1} \mathrm{H}\right\}$ NMR spectrum of 7 in THF-d8. 


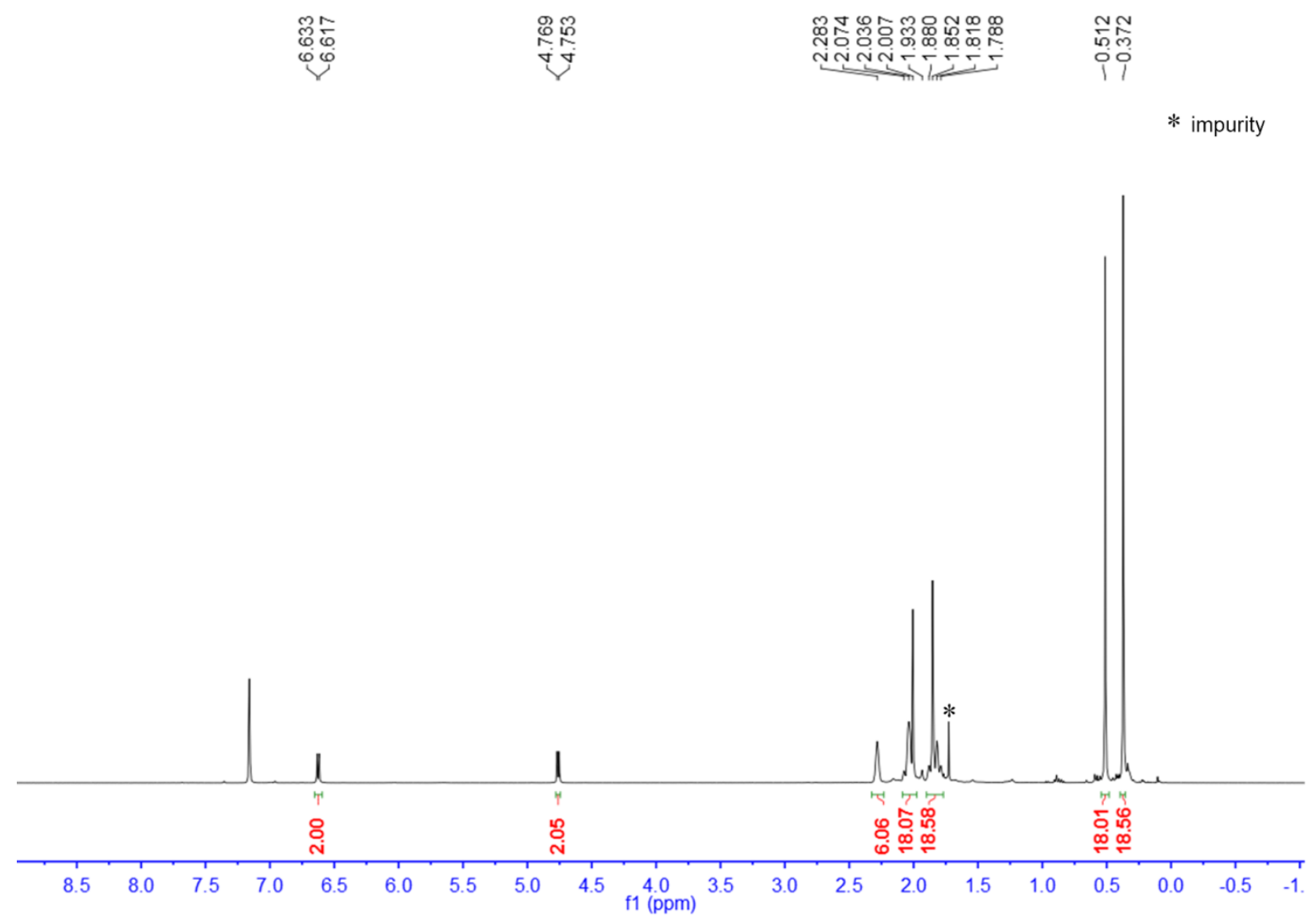

Figure S37. ${ }^{1} \mathrm{H}$ NMR spectrum of 8 in $\mathrm{C}_{6} \mathrm{D}_{6}$.

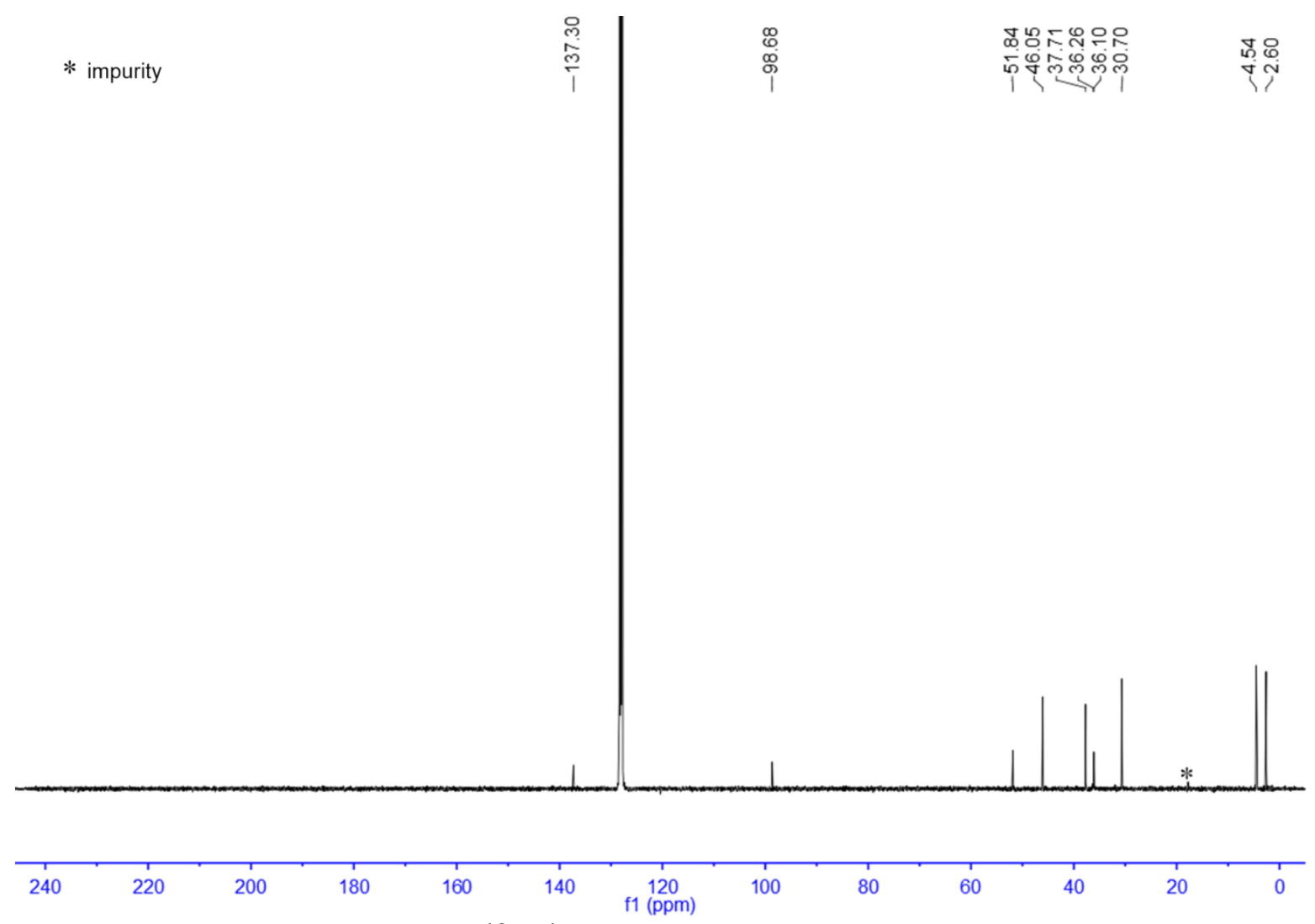

Figure S38. ${ }^{13} \mathrm{C}\left\{{ }^{1} \mathrm{H}\right\}$ NMR spectrum of 8 in $\mathrm{C}_{6} \mathrm{D}_{6}$. 


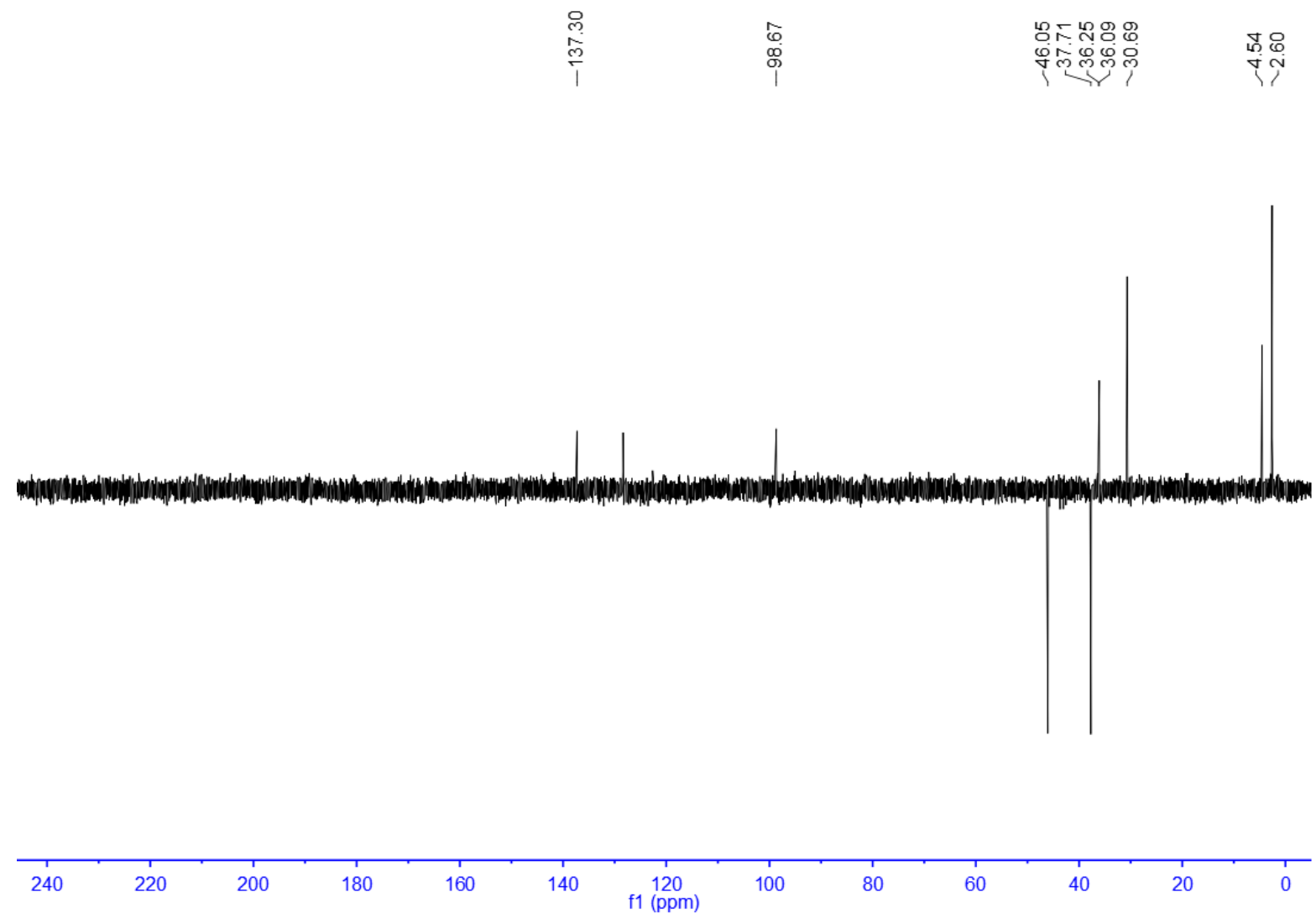

Figure S39. ${ }^{13} \mathrm{C}$ (DEPT 135) NMR spectrum of 8 in $\mathrm{C}_{6} \mathrm{D}_{6}$.

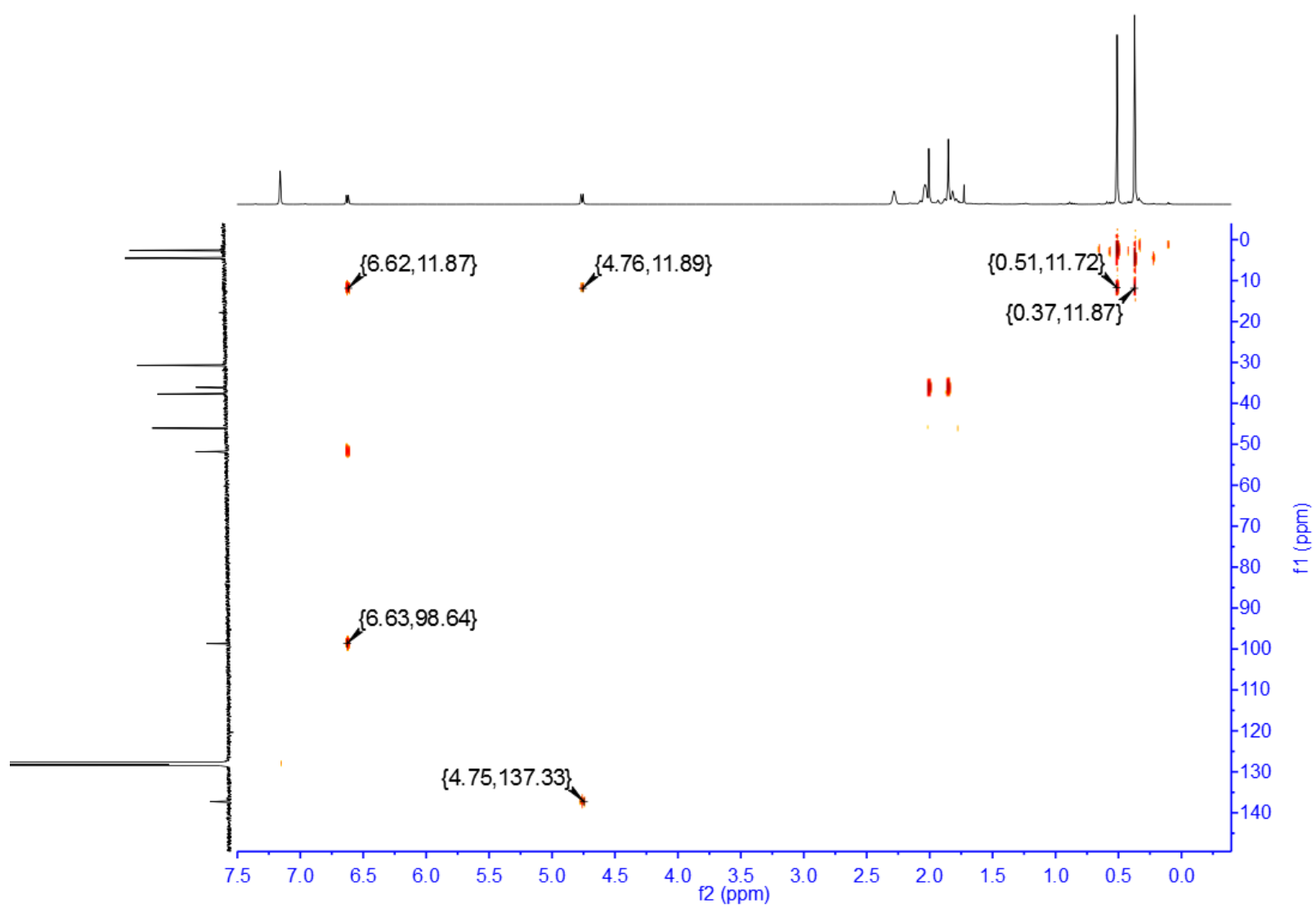

Figure S40. ${ }^{1} \mathrm{H}-{ }^{13} \mathrm{C}$ HMBC NMR spectrum of 8 in $\mathrm{C}_{6} \mathrm{D}_{6}$. 

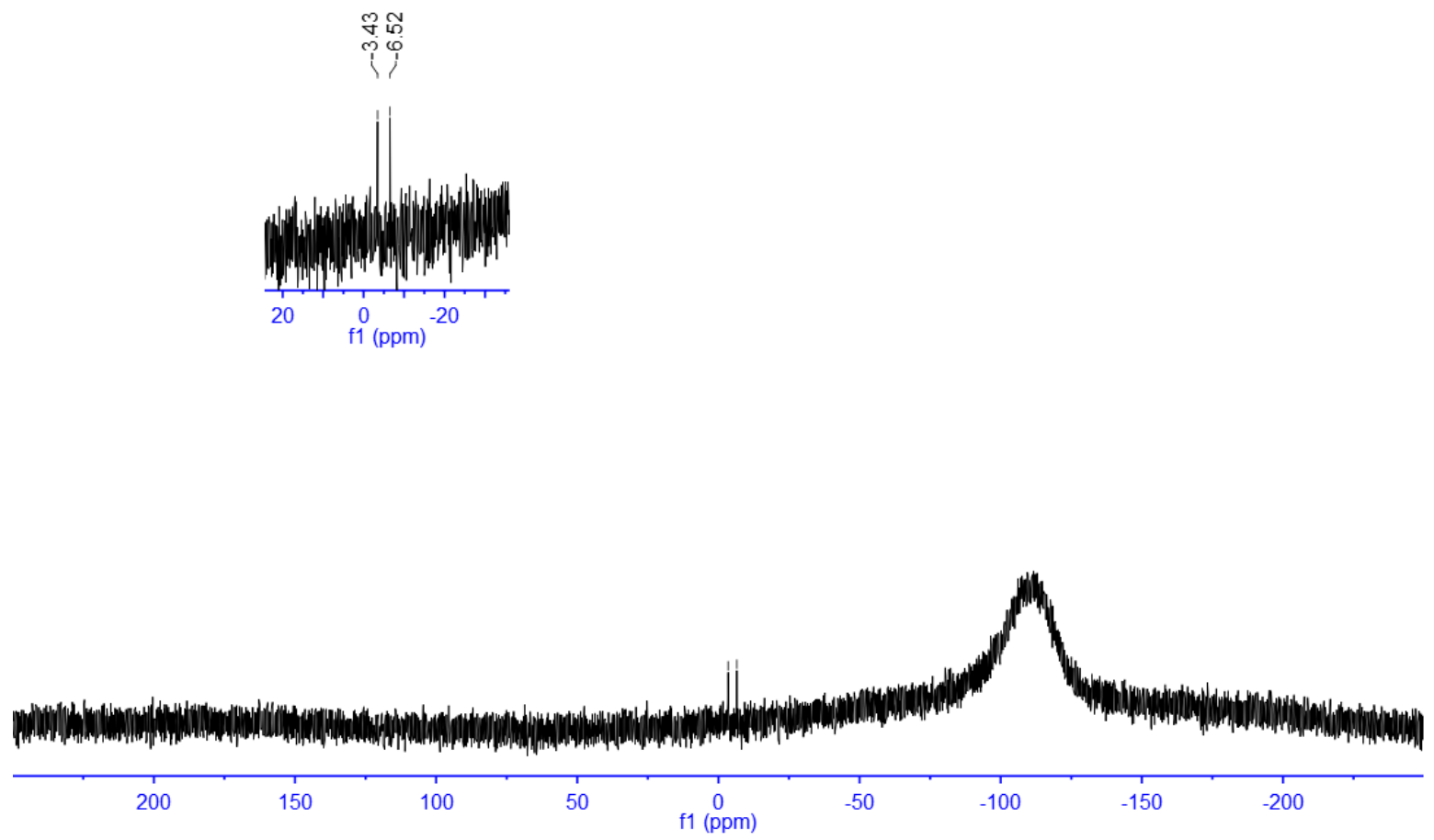

Figure S41. ${ }^{29} \mathrm{Si}\left\{{ }^{1} \mathrm{H}\right\}$ NMR spectrum of 8 in $\mathrm{C}_{6} \mathrm{D}_{6}$. 


\section{Reduction of dialane(4) 1 with $\mathrm{KC}_{8}$, followed by the oxidation with $\mathrm{N}_{2} \mathrm{O}$ gas}

$\mathrm{Et}_{2} \mathrm{O}(1.0 \mathrm{ml})$ was added to a mixture of dialane $(4) 1(40.0 \mathrm{mg}, 0.055 \mathrm{mmol})$ and $\mathrm{KC}_{8}(22.5 \mathrm{mg}$, $0.17 \mathrm{mmol}$ ) at room temperature in a vial. The mixture was stirred for 1 hour and the solution part was separated by filtration. The resulting solution was degassed using a freeze-pumpthaw method, and it was refilled with $\mathrm{N}_{2} \mathrm{O}$ gas at $-78{ }^{\circ} \mathrm{C}$. After gradually warming up to room temperature and stirring for 1 minute at room temperature, volatiles were removed under vacuum. The residual solid was dissolved in THF-d8 and analyzed by ${ }^{1} \mathrm{H}$ NMR (Figure S42).
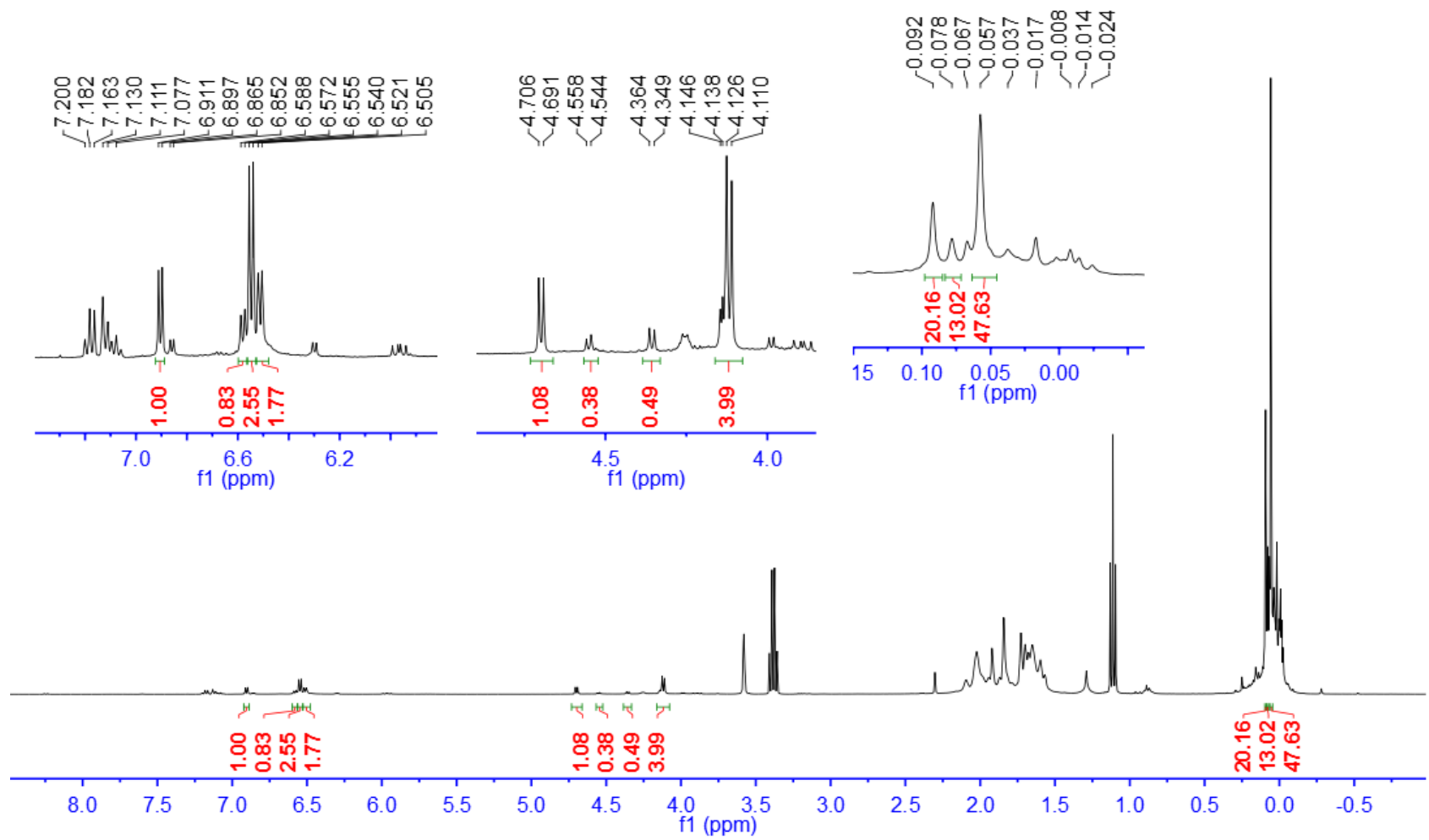

Figure S42. ${ }^{1} \mathrm{H}$ NMR spectrum (in THF-d8) of the crude product after the reaction with $\mathrm{N}_{2} \mathrm{O}$ gas. 


\section{Oxidation of 2 with diphenyl diselenide}

(a)

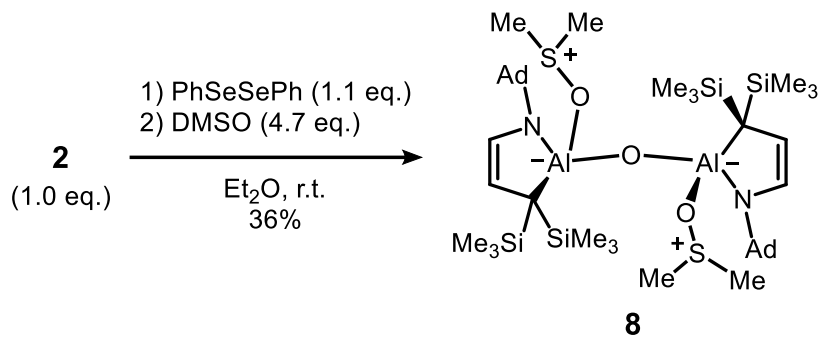

(b)

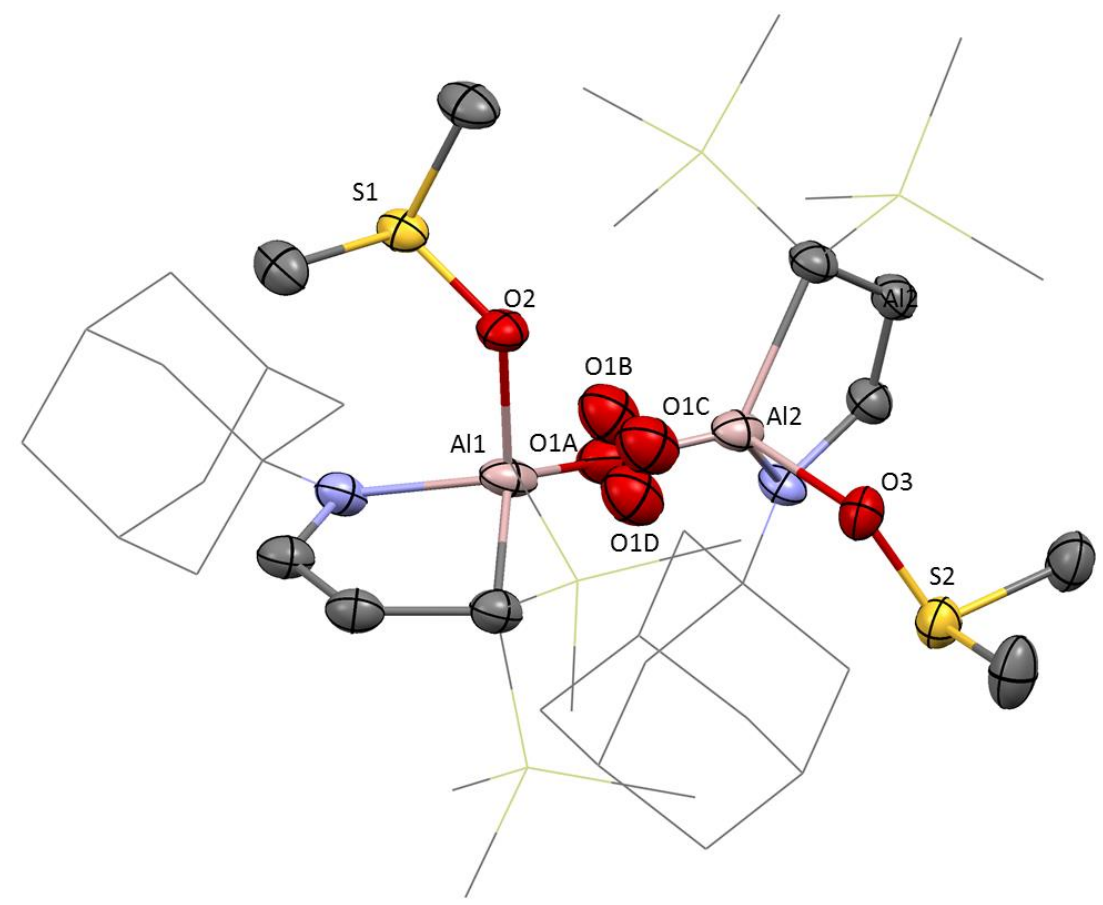

Figure S43. Oxidation of 2 with diphenyl diselenide, followed by treatment with dimethyl sulfoxide. (a) Reaction scheme. (b) The solid-state structure of $\mathbf{8}$. Hydrogen atoms and solvated $\mathrm{Et}_{2} \mathrm{O}$ and DMSO are omitted for clarity. Thermal ellipsoids are shown at the $50 \%$ probability level. 


\section{Crystal structural parameters for $2-7$}

X-ray data collection and structural refinement. Intensity data for compounds 2-7 were collected using a Bruker APEX II diffractometer. The crystals were measured at 100(2) K. The structure was solved by direct phase determination (SHELX-2013) and refined for all data by full-matrix least squares methods on $F^{2}$. All non-hydrogen atoms were subjected to anisotropic refinement. The hydrogen atoms were generated geometrically and allowed to ride in their respective parent atoms; they were assigned appropriate isotropic thermal parameters and included in the structure-factor calculations. CCDC; 2096139-2096144, 2107251 contains the supplementary crystallographic data for this paper. The data can be obtained free of charge from the Cambridge Crystallography Data Center via www.ccdc.cam.ac.uk/data request/cif.

Table S1. Summary of Data Collection and Structure Refinement.

\begin{tabular}{|c|c|c|c|}
\hline Compounds & 2 & 3 & 4-toluene \\
\hline Formula & $\mathrm{C}_{46} \mathrm{H}_{90} \mathrm{Al}_{2} \mathrm{~K}_{2} \mathrm{~N}_{2} \mathrm{O}_{3} \mathrm{Si}_{4}$ & $\mathrm{C}_{55} \mathrm{H}_{91} \mathrm{Al}_{2} \mathrm{~K}_{2} \mathrm{~N}_{3} \mathrm{OSi}_{4}$ & $\mathrm{C}_{54} \mathrm{H}_{90} \mathrm{Al}_{2} \mathrm{~K}_{2} \mathrm{~N}_{2} \mathrm{OSi}_{4}$ \\
\hline $\mathrm{Fw}$ & 963.71 & 1054.82 & 1027.79 \\
\hline Crystal syst & Monoclinic & Triclinic & Monoclinic \\
\hline Space group & $P 121 / n 1$ & $P-1$ & $P 12{ }_{1} / n 1$ \\
\hline Size, $\mathrm{mm}^{3}$ & $0.100 \times 0.300 \times 0.300$ & $0.100 \times 0.250 \times 0.400$ & $0.030 \times 0.060 \times 0.170$ \\
\hline $\mathrm{T}, \mathrm{K}$ & $100(2)$ & $100(2)$ & $100(2)$ \\
\hline$a, \AA$ & $14.5035(8)$ & $12.2843(5)$ & $12.8819(9)$ \\
\hline$b, \AA$ & $19.1332(9)$ & $12.9103(5)$ & $28.306(2)$ \\
\hline$c, \AA$ & $19.5482(11)$ & $20.5357(8)$ & $16.2070(12)$ \\
\hline$\alpha, \operatorname{deg}$ & 90 & $93.1930(10)$ & 90 \\
\hline$\beta$, deg & $94.435(3)$ & $92.9460(10)$ & $102.702(4)$ \\
\hline$\gamma$, deg & 90 & $110.8820(10)$ & 90 \\
\hline $\mathrm{V}, \AA^{3}$ & $5408.4(5)$ & $3029.3(2)$ & $5765.0(7)$ \\
\hline Z & 4 & 2 & 4 \\
\hline$d_{\text {calcd }}, \mathrm{g} \cdot \mathrm{cm}^{-1}$ & 1.184 & 1.156 & 1.184 \\
\hline$\mu, \mathrm{mm}^{-1}$ & 0.334 & 0.302 & 0.315 \\
\hline Refl collected & 33509 & 56501 & 35215 \\
\hline $\mathrm{T}_{\min } / \mathrm{T}_{\max }$ & $0.80 / 0.97$ & $0.90 / 0.97$ & $0.80 / 0.99$ \\
\hline $\mathrm{N}_{\text {measd }}$ & 11874 & 13484 & 11657 \\
\hline$\left[\mathrm{R}_{\text {int }}\right]$ & 0.0689 & 0.0369 & 0.1275 \\
\hline $\mathrm{R}[\mathrm{I}>2$ sigma(I)] & 0.0541 & 0.0435 & 0.0869 \\
\hline $\mathrm{R}_{\mathrm{w}}[\mathrm{l}>2 \operatorname{sigma}(\mathrm{I})]$ & 0.1005 & 0.1074 & 0.1717 \\
\hline GOF & 0.998 & 1.045 & 0.998 \\
\hline $\begin{array}{c}\text { Largest diff. } \\
\text { Peak/hole, } \mathrm{e} \cdot \AA^{-3}\end{array}$ & $0.581 /-0.339$ & $1.277 /-0.848$ & $0.700 /-0.504$ \\
\hline
\end{tabular}




\begin{tabular}{|c|c|c|c|c|}
\hline Compounds & 5 & $(6)_{2}$ toluene & $(7)_{2} \cdot$ hexane & $\left(8 \cdot \mathrm{Et}_{2} \mathrm{O} \cdot \mathrm{DMSO}\right)_{0.5}$ \\
\hline Formula & $\mathrm{C}_{55} \mathrm{H}_{90} \mathrm{Al}_{2} \mathrm{~K}_{2} \mathrm{~N}_{2} \mathrm{O}_{3} \mathrm{Si}_{4}$ & $\mathrm{C}_{139} \mathrm{H}_{200} \mathrm{Al}_{4} \mathrm{~K}_{4} \mathrm{~N}_{8} \mathrm{O}_{2} \mathrm{Si}_{8}$ & $\mathrm{C}_{130} \mathrm{H}_{218} \mathrm{Al}_{4} \mathrm{~K}_{4} \mathrm{~N}_{4} \mathrm{O}_{8} \mathrm{~S}_{8}$ & $\mathrm{C}_{24} \mathrm{H}_{49} \mathrm{AlK}_{2} \mathrm{NO}_{2.5} \mathrm{~S}_{1.5} \mathrm{Si}_{2}$ \\
\hline Fw & 1071.80 & 2504.10 & 2454.11 & 522.89 \\
\hline Crystal syst & Monoclinic & Triclinic & Triclinic & Triclinic \\
\hline Space group & $P 1 n 1$ & $P-1$ & $P-1$ & $P-1$ \\
\hline Size, $\mathrm{mm}^{3}$ & $0.090 \times 0.090 \times 0.120$ & $0.050 \times 0.130 \times 0.260$ & $0.060 \times 0.120 \times 0.250$ & $0.100 \times 0.280 \times 0.370$ \\
\hline $\mathrm{T}, \mathrm{K}$ & $100(2)$ & $100(2)$ & $100(2)$ & $100(2)$ \\
\hline$a, \AA$ & $13.1965(11)$ & $12.9614(5)$ & $16.9856(9)$ & $12.1840(9)$ \\
\hline$b, \AA$ & $9.8871(6)$ & $13.3034(5)$ & $20.5967(11)$ & $14.2787(9)$ \\
\hline$c, \AA$ & $22.6512(16)$ & $22.9220(9)$ & $21.7705(12)$ & $18.1494(12)$ \\
\hline$\alpha, \operatorname{deg}$ & 90 & $101.2490(10)$ & $108.341(2)$ & $83.273(2)$ \\
\hline$\beta, \operatorname{deg}$ & $101.922(5)$ & $96.2590(10)$ & $102.849(2)$ & $73.409(2)$ \\
\hline$\gamma$, deg & 90 & $113.8120(10)$ & $101.851(2)$ & $81.971(2)$ \\
\hline$V, \AA^{3}$ & $2891.7(4)$ & $3468.0(2)$ & $6729.2(6)$ & $2986.4(4)$ \\
\hline z & 2 & 1 & 2 & 4 \\
\hline$d_{\text {calcd }}, \mathrm{g} \cdot \mathrm{cm}^{-1}$ & 1.231 & 1.199 & 1.211 & 1.163 \\
\hline$\mu, \mathrm{mm}^{-1}$ & 0.320 & 0.275 & 0.284 & 0.275 \\
\hline Refl collected & 19004 & 60636 & 124389 & 54366 \\
\hline $\mathrm{T}_{\min } / \mathrm{T}_{\max }$ & $0.84 / 0.97$ & $0.82 / 0.99$ & $0.85 / 0.98$ & $0.85 / 0.97$ \\
\hline $\mathrm{N}_{\text {measd }}$ & 9580 & 14229 & 27507 & 12268 \\
\hline$\left[R_{\text {int }}\right]$ & 0.0727 & 0.0831 & 0.1128 & 0.0843 \\
\hline $\mathrm{R}[\mathrm{I}>2 \operatorname{sigma}(\mathrm{I})]$ & 0.0546 & 0.0542 & 0.0673 & 0.0687 \\
\hline $\mathrm{R}_{\mathrm{w}}[\mathrm{l}>2 \operatorname{sigma}(\mathrm{I})]$ & 0.0879 & 0.1229 & 0.1629 & 0.1690 \\
\hline GOF & 1.023 & 1.069 & 1.029 & 0.972 \\
\hline $\begin{array}{c}\text { Largest diff. } \\
\text { Peak/hole, e } \AA^{-3}\end{array}$ & $0.421 /-0.453$ & $0.770 /-0.617$ & $1.219 /-1.049$ & $1.176 /-0.959$ \\
\hline
\end{tabular}

(a)

(b)
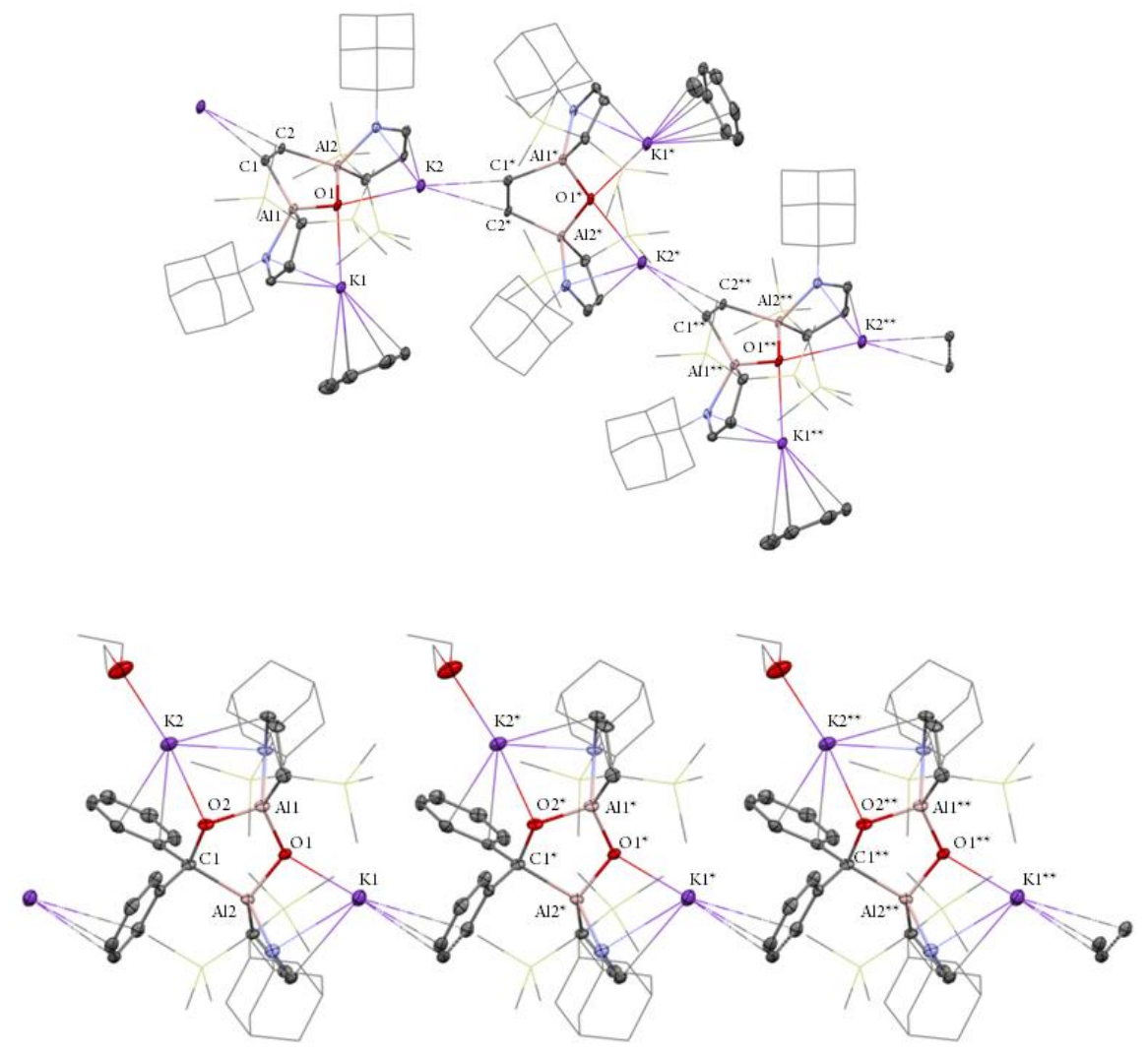

Figure S44. One-dimensional polymer -chain structures of 4 (a) and 5 (b). 


\section{Theoretical calculation}

Gaussian 09 was used for the density functional theory (DFT) calculations. ${ }^{[\mathrm{S} 2]}$ Geometry optimization and frequency calculations were performed with the BP86 functional and hybrid basis sets of TZVP (for $\mathrm{Al}, \mathrm{Si}, \mathrm{K}$ ) and SVP (for $\mathrm{H}, \mathrm{C}, \mathrm{N}, \mathrm{O}$ ). All local minimum and maximum points were confirmed by the frequency calculations to have zero and one imaginary frequencies, respectively. Transition states (TS) except for TS-1, TS-4, and TS-2* were confirmed to connect illustrated ground states by intrinsic reaction coordinate (IRC) calculations. IRC calculations for TS-1, TS-4, and TS-2* did not work fine. Instead, we confirmed that starting structures, which were produced by moving TS structures along with the direction of imaginary vibration, converted into corresponding ground states through geometry optimizations. The starting structures were obtained by GaussView 5.0, following the procedure below. First, open the .log file in GaussView 5.0, and open the vibrations window ("Results" $\rightarrow$ "Vibrations..."). In the vibrations window, select the imaginary frequency, then tick the "Manual Displacement" tab and set the value either to +0.1 or to -0.1 . Finally, click the "Save Structure...", and generate the starting point in a new window.

Natural bond orbital (NBO) analysis were performed using NBO 7.0 program ${ }^{[S 3]}$ with the Gaussian 16 package $^{[\mathrm{S} 4]}$ at the PBE0/def2-TZVP level of theory, with Grimme's empirical dispersion correction (DFT-D3). ${ }^{[55]}$ Multiwfn 3.8 (dev) program ${ }^{[\mathrm{S6}]}$ was used for the topological analysis including the quantum theory of atoms in molecules (QTAIM) and the electron localization function (ELF). The wavefunction files (.wfn) for the topological analysis was obtained from Gaussian 09 at the PBE0-D3/def2-TZVP level of theory using the optimized geometry. 
Table S2. Selected data of Natural Bond Orbital Analysis of 2.

(Occupancy) Bond orbital / Coefficients / Hybrids

110. (1.91111) BD ( 1$)$ Al 1-Al 2

( 49.80\%) $\quad 0.7057^{*} A l 1$ s( $\left.42.72 \%\right)$ p $1.33(56.70 \%) d 0.01(0.48 \%)$ $f 0.00(0.11 \%)$

$0.0000 \quad 0.0000 \quad 0.6536-0.0026-0.0024$

$\begin{array}{lllll}0.0000 & 0.3592 & 0.0031 & -0.0049 & -0.0002\end{array}$

$\begin{array}{lllll}0.0000 & 0.5503 & -0.0036 & -0.0087 & -0.0004\end{array}$

$\begin{array}{llllll}0.0000 & -0.3646 & -0.0434 & 0.0147 & -0.0001\end{array}$

$\begin{array}{lllll}-0.0102 & 0.0401 & 0.0200 & -0.0195 & 0.0214\end{array}$

$\begin{array}{lllll}-0.0195 & 0.0050 & 0.0203 & 0.0029 & -0.0316\end{array}$

$0.0099-0.0087-0.0182-0.0001-0.0016$

$-0.02310 .0073$

$(50.20 \%) \quad 0.7085 * A l \quad 2$ s( $42.99 \%) p 1.31(56.42 \%) d 0.01(0.48 \%)$ f $0.00(0.11 \%)$
0.0000
0.0000
0.6557
$0.0000-0.3807$
$-0.0043$
$-0.0019$
$-0.0025$
$0.0000-0.3576$
0.0209
0.0052
0.0002
0.0000
$-0.5378$
$-0.0379$
0.0022
0.0003
$-0.0012$
0.0308
$-0.02240 .0331-0.0167$
0.0166
0.0001
$0.0402-0.0039$
$0.0200 \quad 0.0043$
$0.0013 \quad 0.0022-0.0160$
$0.0185-0.0086$

105. (1.82535) LP ( 4) 0143

$s(85.69 \%)$ p $0.17(14.25 \%) d 0.00(0.06 \%)$

$f 0.00(0.00 \%)$

$\begin{array}{llllll}0.0000 & 0.9255 & -0.0165 & -0.0009 & 0.0000\end{array}$

$\begin{array}{llllll}0.0017 & 0.0001 & 0.0000 & 0.0716 & 0.0005\end{array}$

$\begin{array}{lllll}-0.0002 & -0.3706 & -0.0014 & 0.0012 & 0.0133\end{array}$

$\begin{array}{lllll}-0.0004 & 0.0033 & -0.0001 & -0.0061 & 0.0002\end{array}$

$\begin{array}{llllll}-0.0037 & 0.0003 & 0.0190 & -0.0006 & -0.0003\end{array}$

$\begin{array}{llllll}-0.0008 & -0.0001 & 0.0007 & -0.0021 & -0.0005\end{array}$

263. (0.15750) LV ( 1)Al 1

$s(4.16 \%)$ p22.91( 95.21\%)d $0.13(0.53 \%)$

$f 0.03(0.11 \%)$

$\begin{array}{llllll}0.0000 & 0.0000 & 0.2035 & -0.0122 & 0.0023\end{array}$

$\begin{array}{lllll}0.0000 & 0.2013 & -0.0025 & 0.0139 & 0.0008\end{array}$

$\begin{array}{lllll}0.0000 & 0.2369 & -0.0187 & 0.0078 & 0.0007\end{array}$

$\begin{array}{lllll}0.0000 & 0.9242 & -0.0224 & 0.0160 & 0.0024\end{array}$

$\begin{array}{llllll}-0.0071 & 0.0079 & -0.0145 & -0.0169 & -0.0362\end{array}$

$\begin{array}{llllll}0.0110 & 0.0107 & -0.0063 & -0.0500 & -0.0236\end{array}$

$\begin{array}{llllll}-0.0064 & 0.0056 & 0.0098 & -0.0043 & 0.0216\end{array}$

$-0.01890 .0085$

264. $(0.15729)$ LV ( 1)Al 2

s( $4.01 \%)$ p $23.76(95.35 \%) d 0.13(0.52 \%)$ $f 0.03(0.11 \%)$
$0.0000 \quad 0.0000$
$\begin{array}{lll}0.1999 & -0.0123 & 0.0024\end{array}$
$0.0000-0.1636$
$0.0019-0.0135-0.0007$
0.0000
$-0.5939$
$0.0246-0.0128-0.0015$
0.0000
0.7569
$\begin{array}{lll}0.0118 & 0.0116\end{array}$
0.0019
$-0.0090$
0.0003
0.0076
0.0169
0.0527
0.0034
0.0307
$-0.0040-0.0169$
$-0.0280$
$-0.0103$
0.0049
$0.0049-0.0105$
0.0095
$0.0271-0.0035$ 
Table S3. Selected WBI values of 2.

Wiberg bond index matrix in the NAO basis:

\begin{tabular}{rccc} 
Atom & 1 & 2 & 143 \\
$-\ldots$ &.---1 &.--1 &..-- \\
1. Al & 0.0000 & 0.9060 & 0.3172 \\
2. Al & 0.9060 & 0.0000 & 0.3140 \\
143. 0 & 0.3172 & 0.3140 & 0.0000 \\
\hline
\end{tabular}

Table S4. Selected data of NPA charges of 2.

\begin{tabular}{|c|c|c|c|c|c|c|}
\hline \multirow{2}{*}{\multicolumn{2}{|c|}{ Atom No }} & \multirow[b]{2}{*}{$\begin{array}{l}\text { Natural } \\
\text { Charge }\end{array}$} & \multicolumn{4}{|c|}{ Natural Population } \\
\hline & & & Core & Valence & Rydberg & Total \\
\hline Al & 1 & 1.32574 & 9.99995 & 1.63669 & 0.03761 & 11.67426 \\
\hline $\mathrm{Al}$ & 2 & 1.31847 & 9.99995 & 1.64407 & 0.03752 & 11.68153 \\
\hline 0143 & & -1.60245 & 2.00000 & 7.58524 & 0.01721 & 9.60245 \\
\hline
\end{tabular}

Table S5. Selected data of Second Order Perturbation Theory Analysis of 2.

SECOND ORDER PERTURBATION THEORY ANALYSIS OF FOCK MATRIX IN NBO BASIS

Threshold for printing: $0.50 \mathrm{kcal} / \mathrm{mol}$

(Intermolecular threshold: $0.05 \mathrm{kcal} / \mathrm{mol}$ )

\begin{tabular}{|c|c|c|c|c|c|}
\hline \multirow{2}{*}{ Donor (L) NBO } & \multirow[b]{2}{*}{ Acceptor (NL) } & & \multicolumn{2}{|c|}{$E(2) E(N L)-E(L$} & \multirow{2}{*}{$\begin{array}{l}F(L, N L) \\
\text { a.u. }\end{array}$} \\
\hline & & NBO & $\mathrm{kcal} / \mathrm{mol}$ & a.u. & \\
\hline$z===========$ & $=============$ & $====$ & $=========$ & $====z$ & $=======$ \\
\hline 105. LP ( 4) 0143 & 263. LV ( 1$) A 1$ & 1 & 54.50 & 0.88 & 0.195 \\
\hline 105. LP ( 4) 0143 & 264. LV ( 1$) A 1$ & 2 & 53.37 & 0.87 & 0.193 \\
\hline
\end{tabular}


(a)

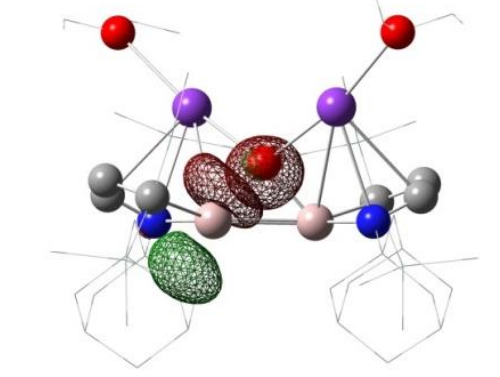

LP of O (105) $\rightarrow$ LP* of Al (263)

$(54.50 \mathrm{kcal} / \mathrm{mol})$ (b)

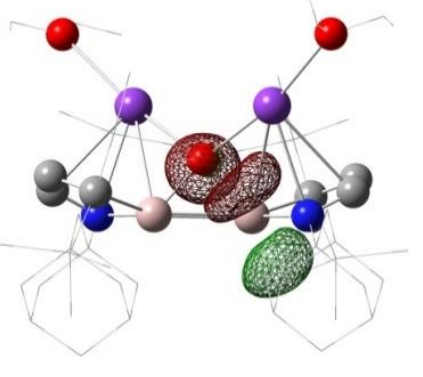

$\mathrm{LP}$ of $\mathrm{O}(105) \rightarrow \mathrm{LP}^{*}$ of $\mathrm{Al}(264)$

(53.37 kcal/mol)

Figure S45. NBO donor-acceptor overlaps in 2 . The values given in parentheses are secondorder perturbation stabilization energies. (a) Overlap between NBO105 and NBO263. (b) Overlap between NBO105 and NBO264. Isovalue $=0.07$. The details of NBOs are described in Table S2.

(a)

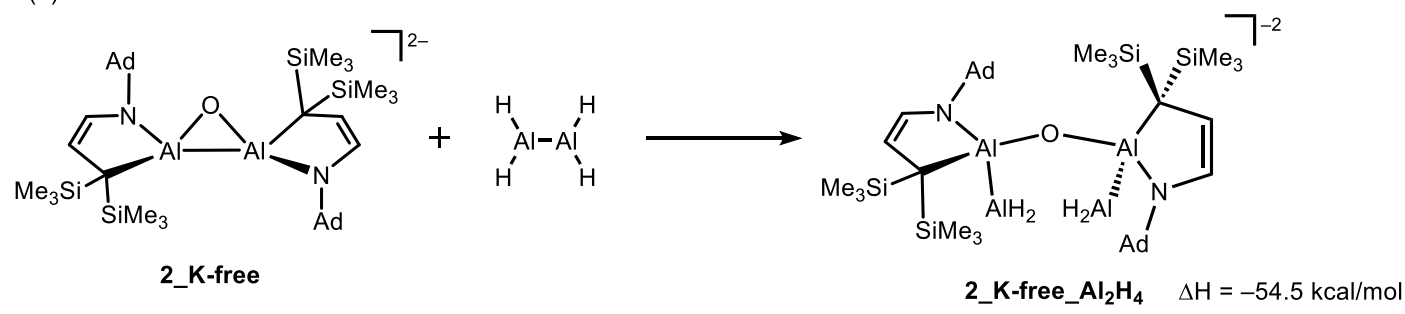

(b)

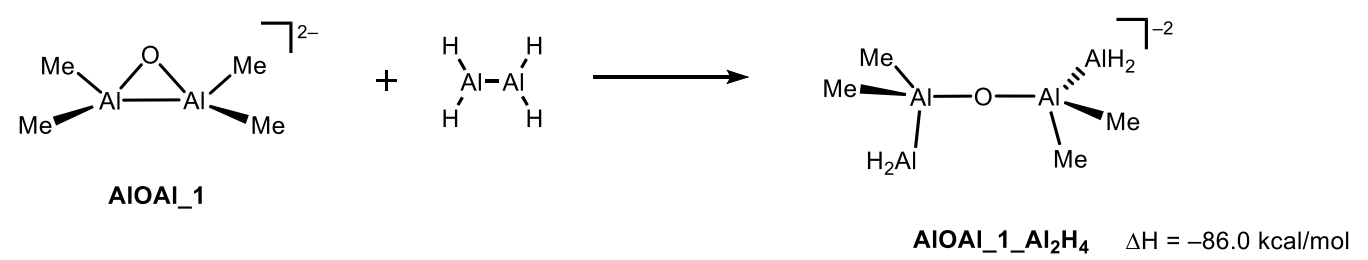

(c)

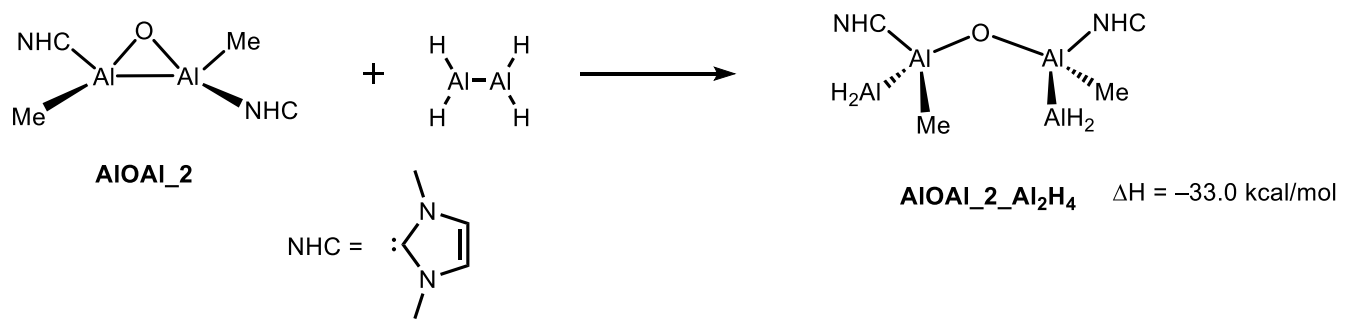

Figure S46. Enthalpy changes of isodesmic reactions by the cleavage of Al-Al bonds. 


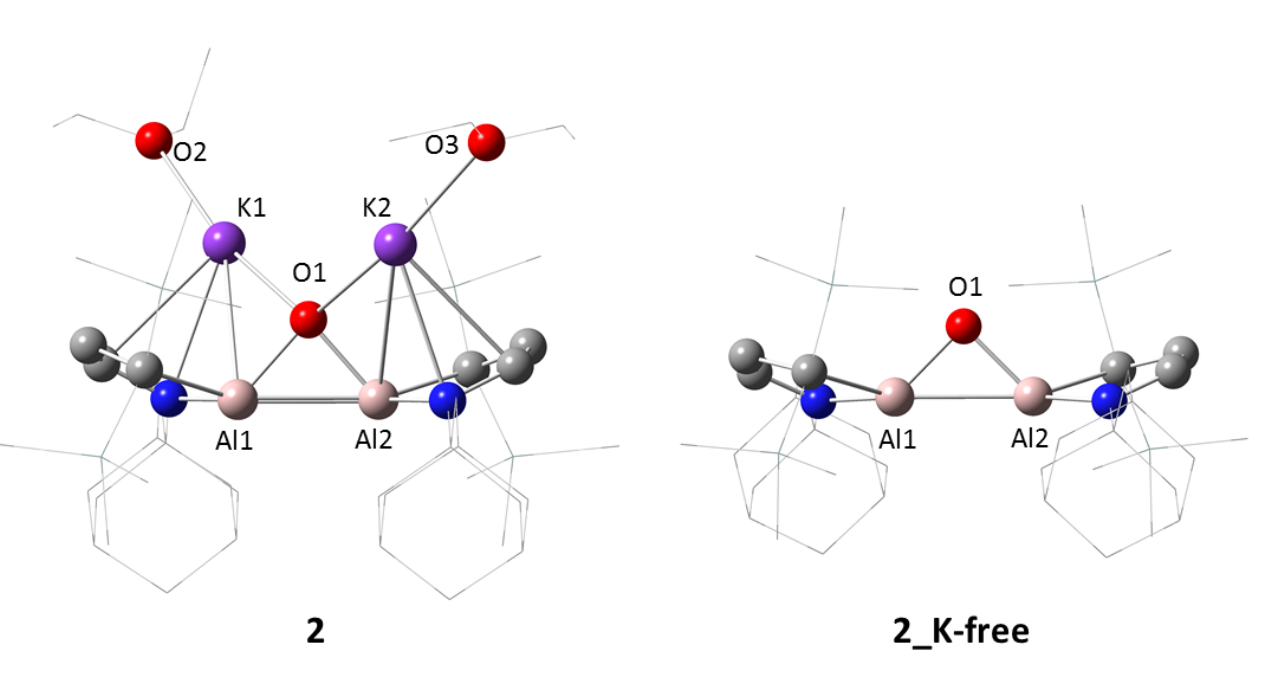

\begin{tabular}{|c|c|c|}
\hline & 2 & 2_K-free \\
\hline Al1-Al2 [Å] & 2.481 & 2.545 \\
\hline Al1-01 [Å] & 1.880 & 1.813 \\
\hline Al2-01 [Å] & 1.869 & 1.813 \\
\hline Al1-01-Al2 [ $\left.{ }^{\circ}\right]$ & 82.9 & 89.2 \\
\hline WBI for Al1-Al2 & 0.91 & 0.91 \\
\hline NPA & $\begin{array}{c}1.33(\mathrm{Al1}), 1.33(\mathrm{Al} 2) \\
-1.60(01)\end{array}$ & $\begin{array}{c}1.31(\mathrm{Al1}), 1.31 \quad(\mathrm{Al2}) \\
-1.54(01)\end{array}$ \\
\hline
\end{tabular}

Figure S47. Counter ion effect on the tricyclic dianionic dialane(6).

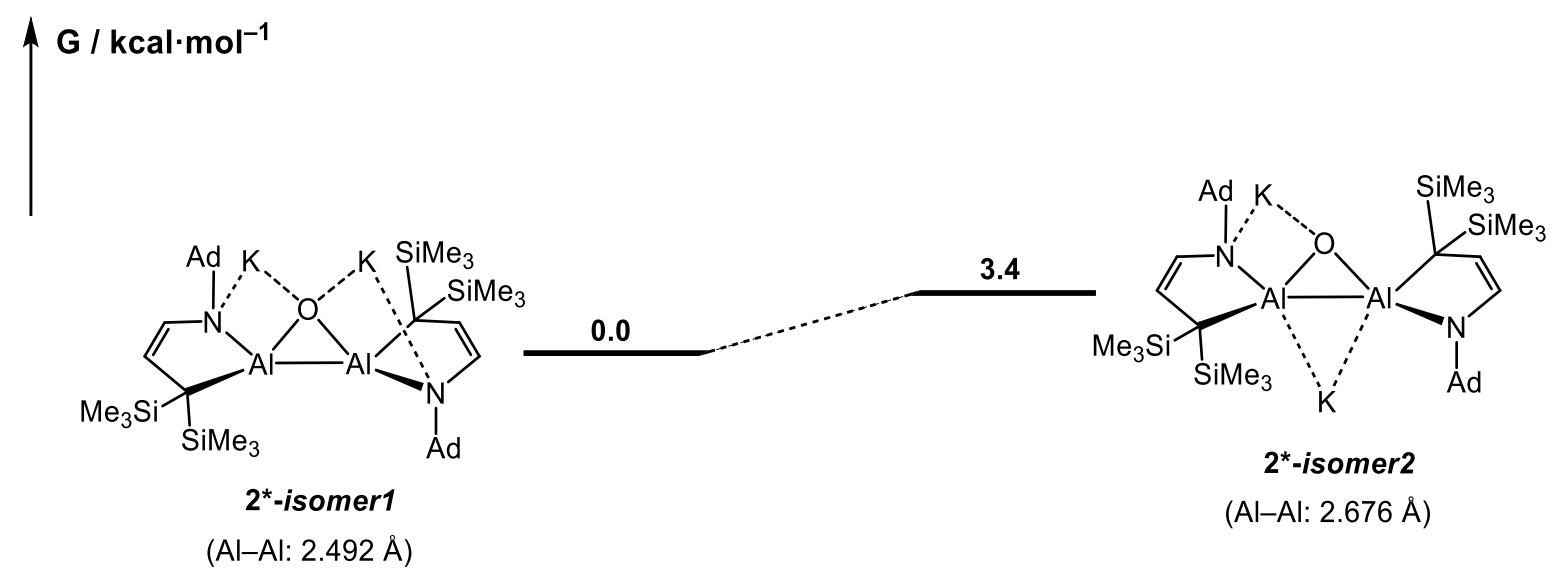

Figure S48. Comparison of free energies between $\mathbf{2}^{*}$-isomer1 and $\mathbf{2}^{*}$-isomer2.

$2^{*}$-isomer1 corresponds to the crystal structure of 2 without $\mathrm{Et}_{2} \mathrm{O}$ molecules on the $\mathrm{K}$ atoms. In $2^{*}$-isomer1, one of the $\mathrm{K}$ cations is located at the outside of the Al-Al bond. The thermal free energy of $2^{*}$-isomer 2 is $3.4 \mathrm{kcal} \cdot \mathrm{mol}^{-1}$ higher than that of $2^{*}$-isomer1. Considering of the small energy gap between $\mathbf{2}^{*}$-isomer 1 and $\mathbf{2}^{*}$-isomer 2 , the position of a $\mathrm{K}$ cation could readily change in the solution state. 

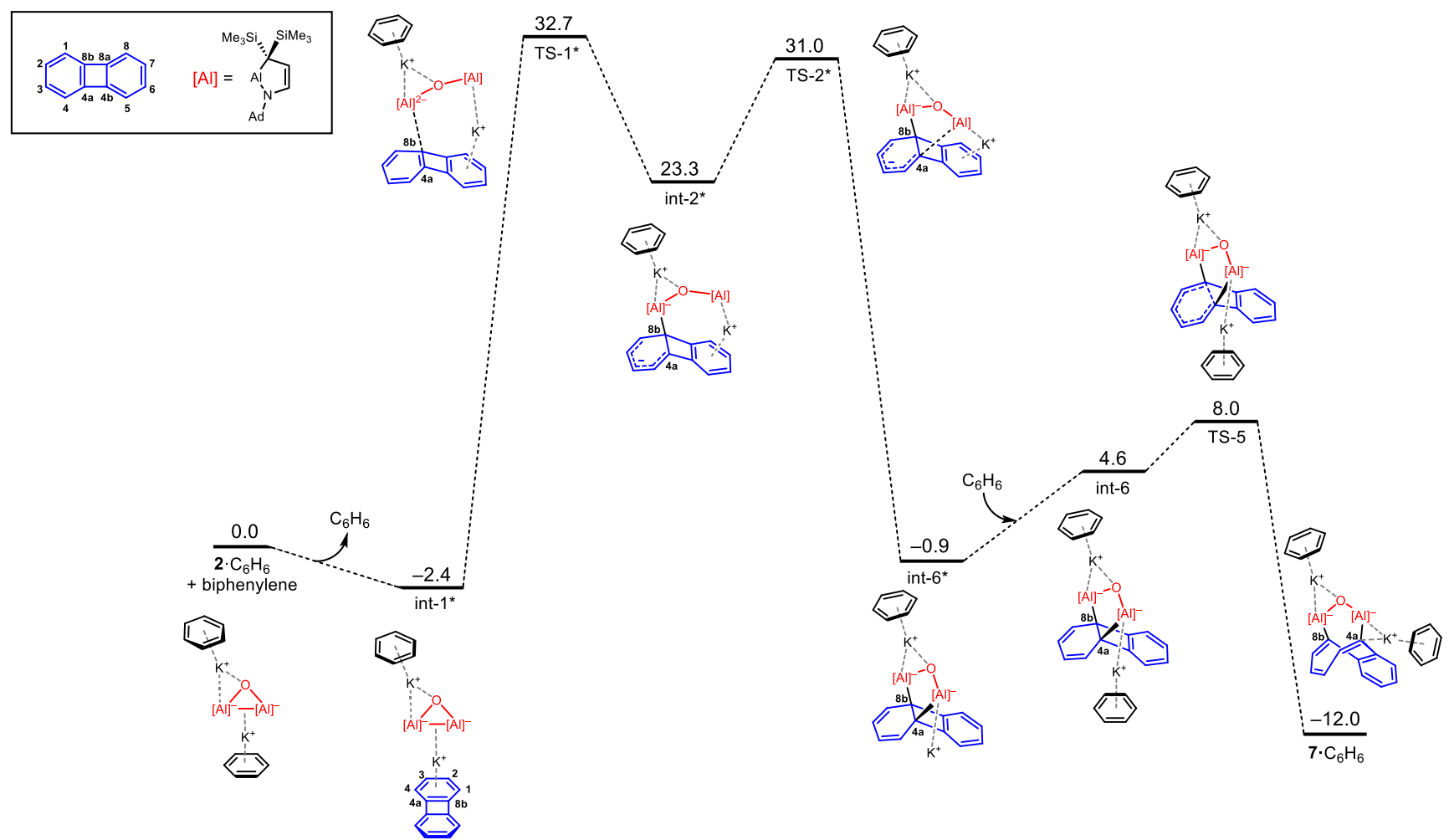

Figure S49. DFT-calculated free energy profile $\left(\mathrm{kcal} \cdot \mathrm{mol}^{-1}\right)$ for the formation of 7 , initiated by the direct addition of the $\mathrm{Al}-\mathrm{Al}$ bond of 2 to the bridging carbon atoms (C4a and $\mathrm{C} 8 \mathrm{~b})$ in biphenylene. The nucleophilic attack of $\mathrm{Al}$ to $\mathrm{C} 8 \mathrm{~b}$ was found to be the rate-determining step with the energy barrier of $35.1 \mathrm{kcal} \cdot \mathrm{mol}^{-1}$. 
Table S6. Cartesian coordinates of 2

Zero-point correction=

Thermal correction to Energy=

Thermal correction to Enthalpy=

Thermal correction to Gibbs Free Energy=

Sum of electronic and zero-point Energies=

Sum of electronic and thermal Energies=

Sum of electronic and thermal Enthalpies=

Sum of electronic and thermal Free Energies $=\mathbf{- 4 9 8 2 . 8 6 1 3 8 6}$
1.244138 (Hartree/Particle)

1.325099

1.326043

1.123296

$-4982.740543$

$-4982.659582$

$-4982.658638$

$-4982.861386$

\begin{tabular}{|c|c|c|c|}
\hline Al & -0.742600 & -0.810900 & -0.891000 \\
\hline Al & 0.695100 & 1.174600 & -0.511100 \\
\hline $\mathrm{C}$ & 2.232000 & -3.280600 & 0.203400 \\
\hline $\mathrm{H}$ & 2.358800 & -2.245000 & -0.173900 \\
\hline $\mathrm{H}$ & 2.814300 & -3.411100 & 1.143800 \\
\hline $\mathrm{H}$ & 2.692000 & -3.947700 & -0.552700 \\
\hline C & -0.040800 & -3.029300 & 2.226000 \\
\hline $\mathrm{H}$ & -1.060000 & -3.345300 & 2.532200 \\
\hline $\mathrm{H}$ & 0.668700 & -3.411200 & 2.992300 \\
\hline $\mathrm{H}$ & 0.005400 & -1.918700 & 2.188100 \\
\hline C & 0.271200 & -5.585400 & 0.611400 \\
\hline $\mathrm{H}$ & 0.659000 & -6.088800 & -0.298600 \\
\hline $\mathrm{H}$ & 0.869200 & -5.949900 & 1.475200 \\
\hline $\mathrm{H}$ & -0.775100 & -5.925300 & 0.761400 \\
\hline $\mathrm{C}$ & 1.243400 & -3.972100 & -3.078700 \\
\hline $\mathrm{H}$ & 1.741300 & -4.760100 & -2.476300 \\
\hline $\mathrm{H}$ & 1.271800 & -4.302200 & -4.139500 \\
\hline $\mathrm{H}$ & 1.847900 & -3.045100 & -3.002100 \\
\hline C & -1.342700 & -2.582200 & -3.903800 \\
\hline$H$ & -0.909900 & -1.559300 & -3.894300 \\
\hline $\mathrm{H}$ & -1.171100 & -3.022100 & -4.910000 \\
\hline $\mathrm{H}$ & -2.437500 & -2.490100 & -3.756900 \\
\hline C & -1.449300 & -5.375500 & -2.709200 \\
\hline $\mathrm{H}$ & -2.534500 & -5.281700 & -2.495700 \\
\hline $\mathrm{H}$ & -1.342800 & -5.766400 & -3.744300 \\
\hline $\mathrm{H}$ & -1.033000 & -6.136900 & -2.017900 \\
\hline C & -0.763500 & -2.919500 & -0.815200 \\
\hline C & -2.214000 & -3.144500 & -0.405300 \\
\hline $\mathrm{H}$ & -2.591100 & -4.142800 & -0.110400 \\
\hline C & -3.121500 & -2.117500 & -0.495300 \\
\hline $\mathrm{H}$ & -4.194500 & -2.318600 & -0.290500 \\
\hline r & -3.766600 & 0.038900 & -1.461500 \\
\hline$C$ & -3.112100 & 1.284200 & -2.104900 \\
\hline $\mathrm{H}$ & -2.360300 & 0.952800 & -2.853000 \\
\hline $\mathrm{H}$ & -2.534600 & 1.839100 & -1.333600 \\
\hline $\mathrm{C}$ & -4.551300 & -0.703400 & -2.586800 \\
\hline $\mathrm{H}$ & -5.040200 & -1.611500 & -2.173400 \\
\hline $\mathrm{H}$ & -3.823000 & -1.053400 & -3.348300 \\
\hline C & -4.811800 & 0.540100 & -0.413100 \\
\hline $\mathrm{H}$ & -4.278700 & 1.111000 & 0.381800 \\
\hline $\mathrm{H}$ & -5.306200 & -0.331300 & 0.074400 \\
\hline $\mathrm{C}$ & -4.166300 & 2.206400 & -2.758700 \\
\hline $\mathrm{H}$ & -3.650300 & 3.087200 & -3.200000 \\
\hline C & -4.918200 & 1.435000 & -3.865700 \\
\hline $\mathrm{H}$ & -4.206300 & 1.107900 & -4.655200 \\
\hline $\mathrm{H}$ & -5.665800 & 2.096800 & -4.357900 \\
\hline C & -5.614400 & 0.207300 & -3.240300 \\
\hline $\mathrm{H}$ & -6.148800 & -0.364700 & -4.031300 \\
\hline $\mathrm{C}$ & -6.624300 & 0.672300 & -2.167300 \\
\hline $\mathrm{H}$ & -7.407700 & 1.315700 & -2.626700 \\
\hline $\mathrm{H}$ & -7.148300 & -0.206200 & -1.727500 \\
\hline C & -5.877300 & 1.455400 & -1.065200 \\
\hline $\mathrm{H}$ & -6.600500 & 1.791500 & -0.288100 \\
\hline$C$ & -5.177000 & 2.682400 & -1.691300 \\
\hline & -5.932000 & 3.359900 & -2.149600 \\
\hline
\end{tabular}




\begin{tabular}{|c|c|c|c|}
\hline $\mathrm{H}$ & -4.654800 & 3.270100 & -0.904300 \\
\hline 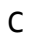 & 0.175300 & 1.891300 & 3.280900 \\
\hline $\mathrm{H}$ & 1.221200 & 2.039500 & 3.625300 \\
\hline $\mathrm{H}$ & -0.478100 & 1.936300 & 4.179400 \\
\hline$\pi$ & 0.083100 & 0.895100 & 2.795100 \\
\hline$c$ & -2.174700 & 2.946200 & 1.639100 \\
\hline $\mathrm{H}$ & -2.323700 & 2.133600 & 0.898400 \\
\hline $\mathrm{H}$ & -2.723500 & 2.708300 & 2.577000 \\
\hline $\mathrm{H}$ & -2.644700 & 3.853700 & 1.211000 \\
\hline C & -0.174300 & 4.881900 & 2.892000 \\
\hline $\mathrm{H}$ & -0.594000 & 5.717000 & 2.293400 \\
\hline $\mathrm{H}$ & -0.730900 & 4.847500 & 3.854000 \\
\hline $\mathrm{H}$ & 0.881300 & 5.136000 & 3.125200 \\
\hline C & 1.229200 & 4.078200 & -2.516300 \\
\hline $\mathrm{H}$ & 0.768400 & 3.166400 & -2.951500 \\
\hline $\mathrm{H}$ & 1.064600 & 4.920100 & -3.223300 \\
\hline $\mathrm{H}$ & 2.321700 & 3.906100 & -2.442200 \\
\hline C & -1.337900 & 4.929700 & -1.114100 \\
\hline $\mathrm{H}$ & -1.820300 & 5.412300 & -0.238400 \\
\hline $\mathrm{H}$ & -1.405000 & 5.646400 & -1.960800 \\
\hline $\mathrm{H}$ & -1.933700 & 4.034600 & -1.387400 \\
\hline C & 1.357000 & 6.104800 & -0.255600 \\
\hline $\mathrm{H}$ & 2.450500 & 5.952900 & -0.141700 \\
\hline $\mathrm{H}$ & 1.205300 & 6.897700 & -1.019600 \\
\hline $\mathrm{H}$ & 0.965200 & 6.486100 & 0.709600 \\
\hline C & 0.759900 & 3.063100 & 0.435500 \\
\hline C & 2.229800 & 3.100900 & 0.835100 \\
\hline $\mathrm{H}$ & 2.635300 & 3.882700 & 1.505900 \\
\hline C & 3.112800 & 2.207000 & 0.281700 \\
\hline $\mathrm{H}$ & 4.197200 & 2.302800 & 0.501400 \\
\hline C & 3.683000 & 0.648500 & -1.513700 \\
\hline C & 4.419500 & 1.788800 & -2.284700 \\
\hline $\mathrm{H}$ & 4.929500 & 2.466100 & -1.566200 \\
\hline $\mathrm{H}$ & 3.658400 & 2.400800 & -2.812700 \\
\hline C & 4.772900 & -0.211600 & -0.797700 \\
\hline $\mathrm{H}$ & 4.277300 & -1.059000 & -0.271000 \\
\hline $\mathrm{H}$ & 5.293100 & 0.401500 & -0.026600 \\
\hline C & 2.995900 & -0.246500 & -2.572400 \\
\hline $\mathrm{H}$ & 2.450200 & -1.069700 & -2.062000 \\
\hline $\mathrm{H}$ & 2.213700 & 0.345900 & -3.093700 \\
\hline C & 5.448300 & 1.228500 & -3.290300 \\
\hline $\mathrm{H}$ & 5.948200 & 2.075100 & -3.811700 \\
\hline C & 6.503800 & 0.390100 & -2.535500 \\
\hline $\mathrm{H}$ & 7.264200 & -0.005400 & -3.245600 \\
\hline $\mathrm{H}$ & 7.049600 & 1.029400 & -1.805400 \\
\hline C & 5.803700 & -0.776700 & -1.803700 \\
\hline $\mathrm{H}$ & 6.560200 & -1.382900 & -1.255800 \\
\hline C & 5.070700 & -1.665900 & -2.833900 \\
\hline $\mathrm{H}$ & 5.800800 & -2.098800 & -3.554100 \\
\hline $\mathrm{H}$ & 4.580500 & -2.523000 & -2.321500 \\
\hline C & 4.016500 & -0.819700 & -3.581500 \\
\hline $\mathrm{H}$ & 3.477700 & -1.459900 & -4.314200 \\
\hline C & 4.722000 & 0.337900 & -4.322000 \\
\hline $\mathrm{H}$ & 5.445800 & -0.063200 & -5.066800 \\
\hline $\mathrm{H}$ & 3.977600 & 0.939800 & -4.888300 \\
\hline C & -5.057400 & -2.510100 & 3.826000 \\
\hline $\mathrm{H}$ & -5.358700 & -2.758700 & 4.873500 \\
\hline $\mathrm{H}$ & -5.984300 & -2.563900 & 3.203200 \\
\hline C & -5.431700 & -0.236900 & 4.391600 \\
\hline $\mathrm{H}$ & -6.415000 & -0.261400 & 3.860100 \\
\hline $\mathrm{H}$ & -5.631200 & -0.539600 & 5.449600 \\
\hline C & -4.015500 & -3.497400 & 3.326800 \\
\hline $\mathrm{H}$ & -3.111200 & -3.482900 & 3.970100 \\
\hline $\mathrm{H}$ & -4.428500 & -4.526200 & 3.351700 \\
\hline $\mathrm{H}$ & -3.712600 & -3.290800 & 2.277200 \\
\hline C & -4.837500 & 1.161400 & 4.343000 \\
\hline $\mathrm{H}$ & -4.699900 & 1.511500 & 3.298700 \\
\hline $\mathrm{H}$ & -5.516200 & 1.881400 & 4.843200 \\
\hline
\end{tabular}




$\begin{array}{lrrr}\text { H } & -3.860000 & 1.201800 & 4.867800 \\ \mathrm{C} & 5.331900 & -2.010800 & 3.505900 \\ \mathrm{H} & 6.383300 & -1.792800 & 3.200500 \\ \mathrm{H} & 4.867900 & -2.581700 & 2.672700 \\ \mathrm{C} & 5.296600 & 0.269400 & 4.272800 \\ \mathrm{H} & 5.586900 & -0.061300 & 5.298000 \\ \mathrm{H} & 6.242900 & 0.496000 & 3.722900 \\ \mathrm{C} & 5.300400 & -2.840300 & 4.789400 \\ \mathrm{H} & 4.257700 & -3.084300 & 5.079600 \\ \mathrm{H} & 5.846200 & -3.794400 & 4.636100 \\ \mathrm{H} & 5.783000 & -2.316600 & 5.640000 \\ \mathrm{C} & 4.413800 & 1.505400 & 4.354300 \\ \mathrm{H} & 3.491500 & 1.301900 & 4.937600 \\ \mathrm{H} & 4.957600 & 2.326400 & 4.863800 \\ \mathrm{H} & 4.123900 & 1.876300 & 3.347300 \\ \mathrm{~K} & 2.402300 & -0.326800 & 1.924000 \\ \mathrm{~K} & -2.363900 & -0.478500 & 2.027900 \\ \mathrm{~N} & -2.728500 & -0.824700 & -0.848200 \\ \mathrm{~N} & 2.680100 & 1.181000 & -0.561200 \\ \mathrm{O} & -0.005000 & -0.104800 & 0.674500 \\ \mathrm{O} & -4.534400 & -1.175800 & 3.792700 \\ \mathrm{O} & 4.595100 & -0.781600 & 3.593500 \\ \mathrm{Si} & 0.379700 & -3.681800 & 0.472900 \\ \mathrm{Si} & -0.570500 & -3.685200 & -2.559700 \\ \mathrm{Si} & -0.311300 & 3.209600 & 1.975400 \\ \mathrm{Si} & 0.495800 & 4.492600 & -0.810700\end{array}$

Table S7. Cartesian coordinates of benzene

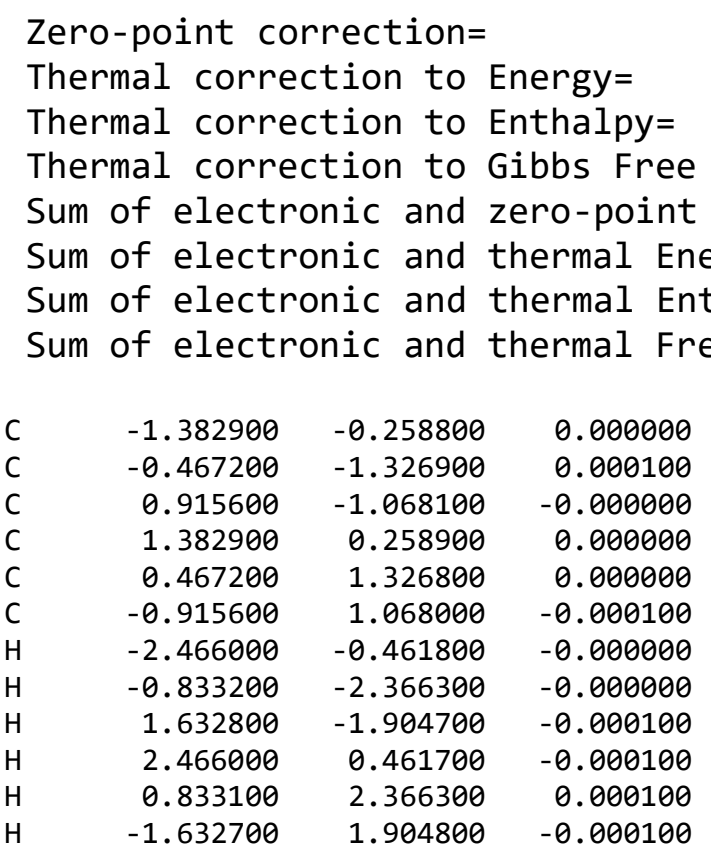

Table S8. Cartesian coordinates of biphenylene

Zero-point correction=

Thermal correction to Energy=

Thermal correction to Enthalpy=

Thermal correction to Gibbs Free Energy=

Sum of electronic and zero-point Energies=

Sum of electronic and thermal Energies=
0.097693 (Hartree/Particle)

0.102199

0.103143

0.070159

$-231.978529$

- 231.974023

$-231.973078$

$-232.006063$ 
Sum of electronic and thermal Enthalpies $=\quad-461.536853$

Sum of electronic and thermal Free Energies= -461.579442

$\begin{array}{lrrr}\text { C } & -0.756100 & -0.717300 & -0.000000 \\ \text { C } & -0.756200 & 0.717300 & -0.000000 \\ \text { C } & -1.927800 & 1.456600 & 0.000000 \\ \text { C } & -3.140600 & 0.700900 & 0.000000 \\ \text { C } & -3.140600 & -0.700900 & 0.000000 \\ \text { C } & -1.927800 & -1.456600 & -0.000000 \\ \text { H } & -1.948800 & 2.557400 & 0.000000 \\ \text { H } & -4.103700 & 1.236100 & 0.000000 \\ \text { H } & -4.103700 & -1.236200 & 0.000000 \\ \text { H } & -1.948800 & -2.557400 & -0.000000 \\ \text { C } & 0.756200 & 0.717300 & 0.000000 \\ \text { C } & 1.927800 & 1.456600 & -0.000000 \\ \text { C } & 0.756100 & -0.717300 & 0.000000 \\ \text { C } & 3.140600 & 0.700900 & -0.000000 \\ \text { H } & 1.948800 & 2.557400 & -0.000000 \\ \text { C } & 1.927800 & -1.456600 & 0.000000 \\ \text { C } & 3.140600 & -0.700900 & 0.000000 \\ \text { H } & 4.103700 & 1.236100 & -0.000000 \\ \text { H } & 1.948800 & -2.557400 & 0.000000 \\ \text { H } & 4.103700 & -1.236200 & 0.000000\end{array}$

Table S9. Cartesian coordinates of $\mathbf{2} \cdot \mathbf{C}_{6} \mathrm{H}_{6}$

Zero-point correction=

Thermal correction to Energy=

1.175247 (Hartree/Particle)

Thermal correction to Enthalpy=

1.251955

Thermal correction to Gibbs Free Energy=

1.252900

Sum of electronic and zero-point Energies=

1.056721

Sum of electronic and thermal Energies $=-4979.908111$

Sum of electronic and thermal Enthalpies $=\quad-4979.907167$

Sum of electronic and thermal Free Energies $=-4980.103345$

$\begin{array}{lrrr}\text { Al } & -0.338000 & -0.164200 & -0.953400 \\ \text { Al } & 0.628700 & -0.117500 & 1.528600 \\ \text { C } & 1.306900 & -4.194800 & -2.428300 \\ \text { H } & 2.063200 & -3.682300 & -1.804600 \\ \text { H } & 1.273400 & -5.260400 & -2.115500 \\ \text { H } & 1.658800 & -4.171300 & -3.480500 \\ \mathrm{C} & -1.087300 & -3.970000 & -0.531700 \\ \text { H } & -2.196700 & -4.070600 & -0.532300 \\ \text { H } & -0.695100 & -4.975600 & -0.269200 \\ \text { H } & -0.775800 & -3.245500 & 0.251300 \\ \mathrm{C} & -1.501200 & -4.426400 & -3.497600 \\ \mathrm{H} & -1.226500 & -4.217100 & -4.551800 \\ \mathrm{H} & -1.357600 & -5.515000 & -3.322400 \\ \mathrm{H} & -2.584600 & -4.210900 & -3.385300 \\ \mathrm{C} & 2.453300 & -1.107400 & -3.631000 \\ \mathrm{H} & 2.832500 & -2.147700 & -3.675700 \\ \mathrm{H} & 3.024700 & -0.509900 & -4.374500 \\ \mathrm{H} & 2.684100 & -0.718200 & -2.616200 \\ \mathrm{C} & 0.265500 & 0.849300 & -4.429100 \\ \mathrm{H} & 0.567000 & 1.524800 & -3.600000 \\ \mathrm{H} & 0.838800 & 1.133000 & -5.338400 \\ \mathrm{H} & -0.810200 & 1.029400 & -4.628500 \\ \mathrm{C} & 0.282000 & -1.932900 & -5.629600 \\ \mathrm{H} & -0.789100 & -1.901400 & -5.919900 \\ \mathrm{H} & 0.865600 & -1.473100 & -6.456500 \\ \mathrm{H} & 0.584500 & -2.999000 & -5.563200 \\ \mathrm{C} & -0.542400 & -1.558500 & -2.561200 \\ \mathrm{C} & -1.971600 & -1.250500 & -2.995000\end{array}$




\begin{tabular}{|c|c|c|c|}
\hline $\mathrm{H}$ & -2.501300 & -1.860500 & -3.749500 \\
\hline C & -2.597100 & -0.116400 & -2.538500 \\
\hline $\mathrm{H}$ & -3.597800 & 0.148900 & -2.936900 \\
\hline C & -2.615400 & 2.051200 & -1.437800 \\
\hline C & -1.745800 & 2.935500 & -0.515800 \\
\hline $\mathrm{H}$ & -0.726400 & 3.011100 & -0.956600 \\
\hline $\mathrm{H}$ & -1.611800 & 2.423200 & 0.461900 \\
\hline C & -2.754000 & 2.803300 & -2.799900 \\
\hline $\mathrm{H}$ & -3.370900 & 2.202800 & -3.502300 \\
\hline $\mathrm{H}$ & -1.744700 & 2.885700 & -3.258200 \\
\hline C & -4.037000 & 1.960800 & -0.795500 \\
\hline $\mathrm{H}$ & -3.947500 & 1.464600 & 0.197800 \\
\hline $\mathrm{H}$ & -4.695200 & 1.326500 & -1.431200 \\
\hline C & -2.367300 & 4.338200 & -0.321700 \\
\hline $\mathrm{H}$ & -1.714100 & 4.934700 & 0.352600 \\
\hline C & -2.491900 & 5.048000 & -1.688200 \\
\hline $\mathrm{H}$ & -1.486100 & 5.180800 & -2.146300 \\
\hline $\mathrm{H}$ & -2.921900 & 6.066200 & -1.555500 \\
\hline C & -3.387100 & 4.201000 & -2.618500 \\
\hline $\mathrm{H}$ & -3.472200 & 4.697200 & -3.611100 \\
\hline C & -4.792300 & 4.052200 & -1.992200 \\
\hline $\mathrm{H}$ & -5.270400 & 5.050500 & -1.876700 \\
\hline $\mathrm{H}$ & -5.449700 & 3.458100 & -2.666100 \\
\hline C & -4.671900 & 3.359000 & -0.616700 \\
\hline $\mathrm{H}$ & -5.682200 & 3.252500 & -0.161500 \\
\hline C & -3.771800 & 4.205400 & 0.309700 \\
\hline $\mathrm{H}$ & -4.218800 & 5.212500 & 0.466500 \\
\hline $\mathrm{H}$ & -3.698700 & 3.729000 & 1.311800 \\
\hline C & -1.315000 & -1.877600 & 3.958200 \\
\hline $\mathrm{H}$ & -0.422300 & -2.247900 & 4.503500 \\
\hline $\mathrm{H}$ & -2.215400 & -2.329100 & 4.429000 \\
\hline $\mathrm{H}$ & -1.238800 & -2.232700 & 2.908700 \\
\hline C & -2.917800 & 0.573100 & 3.005900 \\
\hline $\mathrm{H}$ & -3.270700 & 1.587500 & 3.281300 \\
\hline $\mathrm{H}$ & -2.643100 & 0.592900 & 1.931200 \\
\hline $\mathrm{H}$ & -3.768300 & -0.127900 & 3.159800 \\
\hline C & -1.746500 & 0.495000 & 5.839500 \\
\hline $\mathrm{H}$ & -1.915900 & 1.585600 & 5.961700 \\
\hline $\mathrm{H}$ & -2.656400 & -0.026800 & 6.208400 \\
\hline $\mathrm{H}$ & -0.904800 & 0.206500 & 6.503400 \\
\hline $\mathrm{C}$ & 1.760100 & 3.326200 & 2.370600 \\
\hline $\mathrm{H}$ & 1.340300 & 3.415600 & 1.345100 \\
\hline $\mathrm{H}$ & 2.116800 & 4.331400 & 2.683300 \\
\hline $\mathrm{H}$ & 2.631300 & 2.635000 & 2.359600 \\
\hline C & -1.159200 & 3.673000 & 3.268700 \\
\hline $\mathrm{H}$ & -1.895400 & 3.518200 & 4.085200 \\
\hline $\mathrm{H}$ & -0.914200 & 4.756700 & 3.245200 \\
\hline $\mathrm{H}$ & -1.654100 & 3.413800 & 2.313100 \\
\hline C & 1.004200 & 3.145200 & 5.314400 \\
\hline $\mathrm{H}$ & 2.035300 & 2.795300 & 5.526100 \\
\hline $\mathrm{H}$ & 0.993000 & 4.252500 & 5.412600 \\
\hline $\mathrm{H}$ & 0.340000 & 2.732100 & 6.102200 \\
\hline C & 0.230600 & 0.768300 & 3.370300 \\
\hline $\mathrm{C}$ & 1.369100 & 0.186900 & 4.197100 \\
\hline $\mathrm{H}$ & 1.479000 & 0.404900 & 5.273600 \\
\hline $\mathrm{C}$ & 2.289700 & -0.625900 & 3.599700 \\
\hline $\mathrm{H}$ & 3.124900 & -1.035800 & 4.196900 \\
\hline C & 3.342600 & -1.743900 & 1.718800 \\
\hline C & 4.675400 & -0.935000 & 1.801700 \\
\hline $\mathrm{H}$ & 4.874100 & -0.645600 & 2.856100 \\
\hline $\mathrm{H}$ & 4.541300 & 0.018700 & 1.237700 \\
\hline C & 3.542800 & -3.087400 & 2.490500 \\
\hline $\mathrm{H}$ & 2.600000 & -3.672900 & 2.420100 \\
\hline $\mathrm{H}$ & 3.712900 & -2.886300 & 3.569400 \\
\hline C & 3.106300 & -2.104500 & 0.236300 \\
\hline $\mathrm{H}$ & 2.160000 & -2.681400 & 0.152900 \\
\hline $\mathrm{H}$ & 2.938500 & -1.170300 & -0.345700 \\
\hline C & 5.865100 & -1.737600 & 1.232800 \\
\hline
\end{tabular}




$\begin{array}{lrrr}\text { H } & 6.797200 & -1.134000 & 1.316300 \\ \mathrm{C} & 6.026700 & -3.049800 & 2.034600 \\ \mathrm{H} & 6.896400 & -3.627800 & 1.649600 \\ \mathrm{H} & 6.241400 & -2.819400 & 3.102000 \\ \mathrm{C} & 4.734100 & -3.890900 & 1.920900 \\ \mathrm{H} & 4.852200 & -4.833400 & 2.500600 \\ \mathrm{C} & 4.466100 & -4.222200 & 0.437100 \\ \mathrm{H} & 5.306700 & -4.816100 & 0.013000 \\ \mathrm{H} & 3.550500 & -4.846300 & 0.342500 \\ \mathrm{C} & 4.291600 & -2.905300 & -0.350200 \\ \mathrm{H} & 4.083600 & -3.134000 & -1.418100 \\ \mathrm{C} & 5.588300 & -2.072100 & -0.248800 \\ \mathrm{H} & 6.442900 & -2.635200 & -0.686300 \\ \mathrm{H} & 5.486900 & -1.134100 & -0.841900 \\ \mathrm{~N} & -2.003600 & 0.717800 & -1.598100 \\ \mathrm{~N} & 2.210300 & -0.967100 & 2.249900 \\ \mathrm{O} & -0.695500 & -0.945100 & 0.672000 \\ \mathrm{Si} & -0.432300 & -3.435500 & -2.253300 \\ \mathrm{Si} & 0.577700 & -0.983400 & -3.996000 \\ \mathrm{Si} & -1.389500 & 0.024500 & 4.021400 \\ \mathrm{Si} & 0.420500 & 2.647600 & 3.564100 \\ \mathrm{~K} & 2.671600 & 1.900300 & -0.794100 \\ \mathrm{C} & 4.546700 & 4.799100 & -1.457000 \\ \mathrm{C} & 3.922800 & 4.562900 & -2.698300 \\ \mathrm{C} & 5.544400 & 3.919600 & -0.991300 \\ \mathrm{C} & 4.297500 & 3.448400 & -3.474900 \\ \mathrm{H} & 3.813200 & 3.266400 & -4.447200 \\ \mathrm{C} & 5.920100 & 2.805700 & -1.768700 \\ \mathrm{C} & 5.297300 & 2.570700 & -3.010700 \\ \mathrm{H} & 3.148300 & 5.254700 & -3.066200 \\ \mathrm{H} & 4.260000 & 5.675100 & -0.853500 \\ \mathrm{H} & 6.037200 & 4.108200 & -0.024200 \\ \mathrm{~K} & -3.176500 & -1.546100 & 0.146900 \\ \mathrm{H} & -5.559800 & -3.054600 & 3.101400 \\ \mathrm{H} & -5.739400 & -3.039900 & 2.014800 \\ \mathrm{H} & -6.433400 & -1.962300 & 1.429400 \\ \mathrm{H} & -5.280500 & -4.102100 & 1.210700 \\ \mathrm{C} & -6.394300 & -2.998700 & -1.849800 \\ \mathrm{H} & -6.667500 & -1.946500 & 0.040000 \\ \mathrm{H} & -6.797900 & -1.135100 & 2.059000 \\ \mathrm{H} & -5.515000 & -4.086400 & -0.178500 \\ \mathrm{H} & -6.740800 & -4.946100 & 1.668700 \\ \mathrm{H} & -7.215600 & -1.107100 & -0.764200 \\ \mathrm{H} & -5.160200 & -4.918900 & -0.806400 \\ \mathrm{H} & 2.124100 & -1.410200 \\ \mathrm{H} & & 1.703200 & -3.620900\end{array}$

Table S10. Cartesian coordinates of Int-1

Zero-point correction=

Thermal correction to Energy=

Thermal correction to Enthalpy=

Thermal correction to Gibbs Free Energy=

Sum of electronic and zero-point Energies $=-5441.530840$

Sum of electronic and thermal Energies $=\quad-5441.442768$

Sum of electronic and thermal Enthalpies = -5441.441824

Sum of electronic and thermal Free Energies $=-5441.667224$
1.328170 (Hartree/Particle)

1.416242

1.417186

1.191786

$\begin{array}{lrrr}\text { Al } & -1.004900 & -0.345200 & 1.068200 \\ \text { Al } & -1.151300 & 1.445900 & -0.991500 \\ \text { C } & -2.409300 & 2.515700 & 4.100400 \\ \text { H } & -1.831400 & 2.970000 & 3.272300\end{array}$




\begin{tabular}{|c|c|c|c|}
\hline $\mathrm{H}$ & -3.278000 & 3.175700 & 4.311000 \\
\hline $\mathrm{H}$ & -1.769400 & 2.515500 & 5.006900 \\
\hline C & -4.441600 & 1.000500 & 2.396100 \\
\hline $\mathrm{H}$ & -5.074700 & 0.087000 & 2.331600 \\
\hline $\mathrm{H}$ & -5.115000 & 1.821800 & 2.722100 \\
\hline $\mathrm{H}$ & -4.014800 & 1.251900 & 1.402300 \\
\hline C & -3.944600 & 0.156900 & 5.236200 \\
\hline $\mathrm{H}$ & -3.279900 & 0.072100 & 6.118700 \\
\hline $\mathrm{H}$ & -4.751600 & 0.878500 & 5.490400 \\
\hline $\mathrm{H}$ & -4.423500 & -0.832900 & 5.080900 \\
\hline C & 0.881100 & 1.054900 & 4.252700 \\
\hline $\mathrm{H}$ & 0.336300 & 1.941200 & 4.634400 \\
\hline $\mathrm{H}$ & 1.777100 & 0.910700 & 4.895000 \\
\hline $\mathrm{H}$ & 1.224300 & 1.292300 & 3.223100 \\
\hline C & 0.984600 & -1.950400 & 3.772200 \\
\hline $\mathrm{H}$ & 1.376200 & -1.808800 & 2.742500 \\
\hline $\mathrm{H}$ & 1.845100 & -2.003000 & 4.473200 \\
\hline $\mathrm{H}$ & 0.457500 & -2.925600 & 3.799600 \\
\hline C & -0.629600 & -0.863700 & 6.091000 \\
\hline $\mathrm{H}$ & -1.324900 & -1.723000 & 6.191400 \\
\hline $\mathrm{H}$ & 0.291200 & -1.107700 & 6.664000 \\
\hline $\mathrm{H}$ & -1.101400 & 0.013600 & 6.580200 \\
\hline C & -1.686900 & -0.450700 & 3.081400 \\
\hline C & -2.314600 & -1.837200 & 3.061000 \\
\hline $\mathrm{H}$ & -2.910100 & -2.221400 & 3.911000 \\
\hline C & -2.091700 & -2.691300 & 2.010800 \\
\hline $\mathrm{H}$ & -2.493100 & -3.725000 & 2.060500 \\
\hline C & -0.763900 & -3.416100 & 0.107100 \\
\hline C & 0.212100 & -2.872800 & -0.961600 \\
\hline $\mathrm{H}$ & 0.996900 & -2.278800 & -0.440900 \\
\hline $\mathrm{H}$ & -0.321600 & -2.161300 & -1.626600 \\
\hline C & 0.056300 & -4.383600 & 1.018400 \\
\hline $\mathrm{H}$ & -0.601500 & -4.803600 & 1.808800 \\
\hline $\mathrm{H}$ & 0.836600 & -3.793500 & 1.544000 \\
\hline C & -1.846200 & -4.268600 & -0.632000 \\
\hline $\mathrm{H}$ & -2.420600 & -3.604500 & -1.316900 \\
\hline $\mathrm{H}$ & -2.565200 & -4.685500 & 0.108400 \\
\hline C & 0.855100 & -4.011300 & -1.786000 \\
\hline $\mathrm{H}$ & 1.536700 & -3.568000 & -2.546100 \\
\hline C & 1.653300 & -4.944700 & -0.848900 \\
\hline $\mathrm{H}$ & 2.471500 & -4.379000 & -0.349200 \\
\hline $\mathrm{H}$ & 2.135500 & -5.758900 & -1.435000 \\
\hline C & 0.695500 & -5.533500 & 0.209000 \\
\hline $\mathrm{H}$ & 1.262400 & -6.196800 & 0.899700 \\
\hline C & -0.414600 & -6.346500 & -0.493100 \\
\hline $\mathrm{H}$ & 0.027700 & -7.192700 & -1.065000 \\
\hline $\mathrm{H}$ & -1.096800 & -6.794100 & 0.264000 \\
\hline C & -1.206700 & -5.420800 & -1.443700 \\
\hline $\mathrm{H}$ & -2.007400 & -6.003200 & -1.952700 \\
\hline C & -0.247900 & -4.825600 & -2.498600 \\
\hline $\mathrm{H}$ & 0.208100 & -5.639400 & -3.105700 \\
\hline $\mathrm{H}$ & -0.809700 & -4.173600 & -3.202800 \\
\hline C & -3.661600 & 3.730000 & -1.542800 \\
\hline $\mathrm{H}$ & -2.854500 & 4.488800 & -1.557900 \\
\hline $\mathrm{H}$ & -4.634100 & 4.259700 & -1.639300 \\
\hline $\mathrm{H}$ & -3.642500 & 3.225600 & -0.554100 \\
\hline C & -4.946800 & 1.303800 & -2.837100 \\
\hline $\mathrm{H}$ & -5.036600 & 0.615900 & -3.702700 \\
\hline $\mathrm{H}$ & -4.897900 & 0.698300 & -1.908800 \\
\hline $\mathrm{H}$ & -5.880800 & 1.904500 & -2.788900 \\
\hline C & -3.726700 & 3.488200 & -4.578200 \\
\hline $\mathrm{H}$ & -3.690900 & 2.852500 & -5.486200 \\
\hline $\mathrm{H}$ & -4.721000 & 3.984000 & -4.550000 \\
\hline $\mathrm{H}$ & -2.956700 & 4.279300 & -4.686800 \\
\hline C & 0.211000 & -0.454200 & -4.161100 \\
\hline $\mathrm{H}$ & 0.639500 & -0.597800 & -3.146400 \\
\hline $\mathrm{H}$ & 0.316000 & -1.406000 & -4.724100 \\
\hline $\mathrm{H}$ & 0.809400 & 0.323300 & -4.680100 \\
\hline
\end{tabular}




\begin{tabular}{|c|c|c|c|}
\hline C & -2.610000 & -1.354400 & -3.316800 \\
\hline $\mathrm{H}$ & -3.698600 & -1.142100 & -3.316300 \\
\hline $\mathrm{H}$ & -2.445000 & -2.281000 & -3.906600 \\
\hline $\mathrm{H}$ & -2.282000 & -1.550500 & -2.274900 \\
\hline C & -2.203600 & 0.349600 & -5.831700 \\
\hline $\mathrm{H}$ & -1.696100 & 1.210400 & -6.315600 \\
\hline $\mathrm{H}$ & -1.985800 & -0.553600 & -6.441800 \\
\hline $\mathrm{H}$ & -3.296900 & 0.532300 & -5.883500 \\
\hline C & -1.727800 & 1.643700 & -2.955700 \\
\hline C & -0.771000 & 2.739300 & -3.397600 \\
\hline $\mathrm{H}$ & -0.620700 & 3.005200 & -4.459100 \\
\hline C & -0.107700 & 3.456400 & -2.435500 \\
\hline $\mathrm{H}$ & 0.558200 & 4.291200 & -2.724100 \\
\hline C & 0.303500 & 4.109800 & -0.109600 \\
\hline C & 1.843300 & 4.307200 & -0.259200 \\
\hline $\mathrm{H}$ & 2.082100 & 4.666500 & -1.283800 \\
\hline $\mathrm{H}$ & 2.346500 & 3.319900 & -0.145900 \\
\hline C & -0.365600 & 5.513400 & -0.256700 \\
\hline $\mathrm{H}$ & -1.463400 & 5.392000 & -0.136700 \\
\hline $\mathrm{H}$ & -0.196700 & 5.904500 & -1.283400 \\
\hline C & 0.022600 & 3.615100 & 1.326000 \\
\hline $\mathrm{H}$ & -1.076200 & 3.489800 & 1.444900 \\
\hline $\mathrm{H}$ & 0.458000 & 2.599300 & 1.461300 \\
\hline C & 2.394000 & 5.300100 & 0.788500 \\
\hline $\mathrm{H}$ & 3.493000 & 5.411700 & 0.649700 \\
\hline C & 1.706500 & 6.671600 & 0.603500 \\
\hline $\mathrm{H}$ & 2.110300 & 7.403200 & 1.338400 \\
\hline $\mathrm{H}$ & 1.929100 & 7.077700 & -0.408600 \\
\hline C & 0.181300 & 6.510600 & 0.790100 \\
\hline $\mathrm{H}$ & -0.317300 & 7.495800 & 0.652100 \\
\hline C & -0.109100 & 5.973800 & 2.209800 \\
\hline $\mathrm{H}$ & 0.264900 & 6.688300 & 2.977100 \\
\hline $\mathrm{H}$ & -1.205900 & 5.878300 & 2.367000 \\
\hline C & 0.571900 & 4.598600 & 2.382700 \\
\hline $\mathrm{H}$ & 0.356000 & 4.198000 & 3.396500 \\
\hline C & 2.098200 & 4.757400 & 2.204200 \\
\hline $\mathrm{H}$ & 2.506600 & 5.448300 & 2.975000 \\
\hline $\mathrm{H}$ & 2.604600 & 3.777000 & 2.354100 \\
\hline $\mathrm{N}$ & -1.365300 & -2.304400 & 0.879900 \\
\hline $\mathrm{N}$ & -0.239200 & 3.144800 & -1.081200 \\
\hline 0 & -2.253800 & 0.610000 & 0.102900 \\
\hline Si & -3.031000 & 0.769100 & 3.667300 \\
\hline $\mathrm{Si}$ & -0.186700 & -0.527800 & 4.261400 \\
\hline $\mathrm{Si}$ & -3.451600 & 2.494700 & -2.972800 \\
\hline $\mathrm{Si}$ & -1.606600 & 0.102600 & -4.035500 \\
\hline K & 2.485800 & 0.445600 & -0.270700 \\
\hline C & 4.277600 & -0.244600 & 2.450000 \\
\hline C & 5.241000 & 0.781700 & 2.433800 \\
\hline C & 4.510400 & -1.507600 & 1.814000 \\
\hline C & 6.501700 & 0.623300 & 1.779900 \\
\hline $\mathrm{H}$ & 7.246400 & 1.434700 & 1.791600 \\
\hline C & 5.736800 & -1.655200 & 1.181300 \\
\hline C & 6.720600 & -0.599800 & 1.163600 \\
\hline $\mathrm{H}$ & 5.022900 & 1.725500 & 2.958800 \\
\hline $\mathrm{H}$ & 3.336600 & -0.090200 & 3.002000 \\
\hline $\mathrm{H}$ & 3.755100 & -2.307700 & 1.871800 \\
\hline C & 6.672300 & -2.538300 & 0.399900 \\
\hline C & 7.654600 & -1.486100 & 0.383700 \\
\hline C & 8.891500 & -1.641000 & -0.227900 \\
\hline $\mathrm{H}$ & 9.660500 & -0.852800 & -0.244400 \\
\hline C & 6.900200 & -3.769800 & -0.198400 \\
\hline C & 8.168200 & -3.930900 & -0.832100 \\
\hline C & 9.128700 & -2.904900 & -0.845800 \\
\hline $\mathrm{H}$ & 10.094100 & -3.083500 & -1.345800 \\
\hline $\mathrm{H}$ & 6.164000 & -4.588600 & -0.193300 \\
\hline $\mathrm{H}$ & 8.403600 & -4.889300 & -1.322000 \\
\hline K & -4.076500 & -1.301700 & 0.110300 \\
\hline $\mathrm{H}$ & -7.996500 & -0.363800 & 0.529600 \\
\hline
\end{tabular}




$\begin{array}{lrrr}\mathrm{C} & -7.554000 & -1.337200 & 0.265200 \\ \mathrm{C} & -7.321800 & -1.661800 & -1.086200 \\ \mathrm{C} & -7.222600 & -2.258300 & 1.278600 \\ \mathrm{C} & -6.758500 & -2.908600 & -1.423800 \\ \mathrm{H} & -7.581900 & -0.942000 & -1.878100 \\ \mathrm{C} & -6.658600 & -3.504500 & 0.941100 \\ \mathrm{H} & -7.406800 & -2.005400 & 2.334600 \\ \mathrm{H} & -6.583500 & -3.165700 & -2.480600 \\ \mathrm{C} & -6.426800 & -3.829800 & -0.410200 \\ \mathrm{H} & -6.403200 & -4.225400 & 1.733700 \\ \mathrm{H} & -5.992600 & -4.806900 & -0.674700 \\ \mathrm{C} & 5.021800 & -0.260100 & -2.968900 \\ \mathrm{C} & 3.821000 & 0.282800 & -3.466000 \\ \mathrm{C} & 3.528200 & 1.648300 & -3.271400 \\ \mathrm{C} & 4.445700 & 2.469700 & -2.586000 \\ \mathrm{C} & 5.645600 & 1.925500 & -2.088600 \\ \mathrm{C} & 5.933000 & 0.560100 & -2.276200 \\ \mathrm{H} & 5.253400 & -1.325800 & -3.124400 \\ \mathrm{H} & 3.108100 & -0.354500 & -4.012700 \\ \mathrm{H} & 2.582200 & 2.071400 & -3.649200 \\ \mathrm{H} & 4.223800 & 3.538900 & -2.442900 \\ \mathrm{H} & 6.361900 & 2.569000 & -1.553600 \\ \mathrm{H} & 6.867900 & 0.130600 & -1.883800\end{array}$

Table S11. Cartesian coordinates of TS-1

Zero-point correction= Thermal correction to Energy= 1.327603 (Hartree/Particle) Thermal correction to Enthalpy= 1.415035 Thermal correction to Gibbs Free 1.415979 Sum of electronic and zero-point Energies $=\quad-5441.529427$ Sum of electronic and thermal Energies $=\quad-5441.441995$ Sum of electronic and thermal Enthalpies $=\quad-5441.441051$ Sum of electronic and thermal Free Energies $=-5441.665247$

$\begin{array}{lrrr}\text { Al } & -0.685600 & -0.486000 & 1.046200 \\ \text { Al } & -1.658500 & 1.723500 & -0.897500 \\ \text { C } & -1.912000 & 1.973400 & 4.448000 \\ \text { H } & -1.347700 & 2.482700 & 3.641300 \\ \text { H } & -2.756400 & 2.631700 & 4.743900 \\ \text { H } & -1.241400 & 1.880600 & 5.326800 \\ \text { C } & -4.094400 & 0.662800 & 2.776100 \\ \text { H } & -4.770400 & -0.216500 & 2.680700 \\ \text { H } & -4.703400 & 1.465300 & 3.244500 \\ \text { H } & -3.762700 & 1.008300 & 1.774800 \\ \text { C } & -3.382400 & -0.507500 & 5.442000 \\ \text { H } & -2.662900 & -0.666800 & 6.269800 \\ \text { H } & -4.188900 & 0.157800 & 5.821300 \\ \text { H } & -3.846100 & -1.489700 & 5.209100 \\ \text { C } & 1.329400 & 0.577800 & 4.249600 \\ \text { H } & 0.800000 & 1.381000 & 4.799400 \\ \text { H } & 2.279100 & 0.375000 & 4.791000 \\ \text { H } & 1.576400 & 0.967900 & 3.239300 \\ \text { C } & 1.461500 & -2.345200 & 3.375200 \\ \text { H } & 1.796700 & -2.050200 & 2.358000 \\ \text { H } & 2.356900 & -2.478300 & 4.019700 \\ \text { H } & 0.948100 & -3.324300 & 3.289100 \\ \text { C } & -0.004400 & -1.601000 & 5.917600 \\ \text { H } & -0.660900 & -2.495100 & 5.957600 \\ \text { H } & 0.961800 & -1.872400 & 6.395800 \\ \text { H } & -0.470300 & -0.808500 & 6.539300 \\ \text { C } & -1.290300 & -0.852600 & 3.070800 \\ \text { C } & -1.856800 & -2.258900 & 2.947400\end{array}$




\begin{tabular}{|c|c|c|c|}
\hline H & -2.323400 & -2.781300 & 3.805100 \\
\hline C & -1.680800 & -2.978000 & 1.789700 \\
\hline $\mathrm{H}$ & -1.989800 & -4.043600 & 1.763800 \\
\hline C & -0.634300 & -3.354100 & -0.380600 \\
\hline C & -0.025700 & -2.582700 & -1.576100 \\
\hline $\mathrm{H}$ & 0.785500 & -1.924200 & -1.193400 \\
\hline $\mathrm{H}$ & -0.790800 & -1.898100 & -2.001600 \\
\hline C & 0.477400 & -4.292500 & 0.182500 \\
\hline $\mathrm{H}$ & 0.075900 & -4.867600 & 1.045000 \\
\hline $\mathrm{H}$ & 1.296900 & -3.658100 & 0.584000 \\
\hline C & -1.773600 & -4.259400 & -0.955100 \\
\hline $\mathrm{H}$ & -2.563500 & -3.608900 & -1.398300 \\
\hline $\mathrm{H}$ & -2.248700 & -4.842100 & -0.135800 \\
\hline C & 0.512400 & -3.536800 & -2.664300 \\
\hline $\mathrm{H}$ & 0.937400 & -2.932900 & -3.497000 \\
\hline C & 1.610400 & -4.442300 & -2.063500 \\
\hline $\mathrm{H}$ & 2.460200 & -3.823100 & -1.697500 \\
\hline $\mathrm{H}$ & 2.021300 & -5.121500 & -2.844000 \\
\hline C & 1.012600 & -5.259600 & -0.896400 \\
\hline $\mathrm{H}$ & 1.799100 & -5.910000 & -0.452700 \\
\hline C & -0.147700 & -6.135100 & -1.421300 \\
\hline $\mathrm{H}$ & 0.223800 & -6.854600 & -2.184900 \\
\hline $\mathrm{H}$ & -0.573900 & -6.740900 & -0.590700 \\
\hline C & -1.240000 & -5.230000 & -2.034700 \\
\hline $\mathrm{H}$ & -2.077900 & -5.859300 & -2.410400 \\
\hline C & -0.638600 & -4.415800 & -3.201500 \\
\hline $\mathrm{H}$ & -0.266200 & -5.099300 & -3.997200 \\
\hline $\mathrm{H}$ & -1.422200 & -3.777400 & -3.666800 \\
\hline C & -4.408000 & 3.730800 & -0.580300 \\
\hline $\mathrm{H}$ & -3.666400 & 4.550400 & -0.673300 \\
\hline $\mathrm{H}$ & -5.404400 & 4.190200 & -0.403400 \\
\hline $\mathrm{H}$ & -4.151100 & 3.136000 & 0.322400 \\
\hline C & -5.700300 & 1.264700 & -1.842400 \\
\hline $\mathrm{H}$ & -5.897600 & 0.658100 & -2.750100 \\
\hline $\mathrm{H}$ & -5.371100 & 0.582100 & -1.032200 \\
\hline $\mathrm{H}$ & -6.665900 & 1.710100 & -1.519700 \\
\hline C & -5.201200 & 3.746200 & -3.534200 \\
\hline $\mathrm{H}$ & -5.304900 & 3.199600 & -4.493800 \\
\hline $\mathrm{H}$ & -6.213400 & 4.087900 & -3.228100 \\
\hline $\mathrm{H}$ & -4.583100 & 4.647900 & -3.721100 \\
\hline C & -0.672500 & 0.435100 & -4.295200 \\
\hline $\mathrm{H}$ & -0.153100 & 0.038300 & -3.396300 \\
\hline $\mathrm{H}$ & -0.529800 & -0.289800 & -5.124800 \\
\hline $\mathrm{H}$ & -0.184200 & 1.391200 & -4.577800 \\
\hline C & -3.269100 & -0.933400 & -3.379200 \\
\hline $\mathrm{H}$ & -4.360400 & -0.863100 & -3.195300 \\
\hline $\mathrm{H}$ & -3.107800 & -1.707300 & -4.159700 \\
\hline $\mathrm{H}$ & -2.770200 & -1.287800 & -2.453000 \\
\hline C & -3.344500 & 1.199500 & -5.582500 \\
\hline $\mathrm{H}$ & -2.998500 & 2.189100 & -5.947400 \\
\hline $\mathrm{H}$ & -3.096600 & 0.450000 & -6.364800 \\
\hline $\mathrm{H}$ & -4.450800 & 1.239600 & -5.499200 \\
\hline C & -2.673000 & 2.079500 & -2.609800 \\
\hline C & -1.956300 & 3.345700 & -3.056400 \\
\hline $\mathrm{H}$ & -2.124800 & 3.794700 & -4.050400 \\
\hline C & -1.078100 & 3.945900 & -2.197200 \\
\hline $\mathrm{H}$ & -0.552200 & 4.872400 & -2.487700 \\
\hline C & 0.028200 & 4.197500 & -0.018400 \\
\hline C & 1.467100 & 4.397900 & -0.580300 \\
\hline $\mathrm{H}$ & 1.421900 & 4.912800 & -1.564900 \\
\hline $\mathrm{H}$ & 1.914300 & 3.395900 & -0.772800 \\
\hline C & -0.589800 & 5.606300 & 0.241400 \\
\hline $\mathrm{H}$ & -1.615500 & 5.472600 & 0.649600 \\
\hline $\mathrm{H}$ & -0.696700 & 6.159000 & -0.717000 \\
\hline C & 0.138900 & 3.482900 & 1.345800 \\
\hline $\mathrm{H}$ & -0.883800 & 3.347200 & 1.762100 \\
\hline "I & 0.544800 & 2.454600 & 1.194400 \\
\hline & 2.341300 & 5.210800 & 0.399800 \\
\hline
\end{tabular}




\begin{tabular}{|c|c|c|c|}
\hline $\mathrm{H}$ & 3.360200 & 5.337800 & -0.030400 \\
\hline C & 1.698800 & 6.599200 & 0.625700 \\
\hline $\mathrm{H}$ & 2.331300 & 7.205800 & 1.311300 \\
\hline $\mathrm{H}$ & 1.643800 & 7.155600 & -0.336600 \\
\hline C & 0.284100 & 6.421800 & 1.222000 \\
\hline $\mathrm{H}$ & -0.180800 & 7.420000 & 1.380800 \\
\hline C & 0.384500 & 5.674200 & 2.569900 \\
\hline $\mathrm{H}$ & 0.998600 & 6.259700 & 3.290200 \\
\hline $\mathrm{H}$ & -0.624800 & 5.562800 & 3.023400 \\
\hline C & 1.015000 & 4.283300 & 2.334200 \\
\hline $\mathrm{H}$ & 1.074900 & 3.728300 & 3.295200 \\
\hline C & 2.433100 & 4.459500 & 1.746300 \\
\hline $\mathrm{H}$ & 3.079100 & 5.020000 & 2.458000 \\
\hline $\mathrm{H}$ & 2.913200 & 3.464700 & 1.599800 \\
\hline $\mathrm{N}$ & -1.127700 & -2.408400 & 0.642400 \\
\hline $\mathrm{N}$ & -0.808300 & 3.400400 & -0.935000 \\
\hline 0 & -2.055000 & 0.486800 & 0.236300 \\
\hline Si & -2.580400 & 0.278600 & 3.890500 \\
\hline $\mathrm{Si}$ & 0.302000 & -1.026800 & 4.118700 \\
\hline Si & -4.439500 & 2.669300 & -2.160300 \\
\hline $\mathrm{Si}$ & -2.517600 & 0.731900 & -3.929600 \\
\hline K & 2.707900 & 0.353400 & -0.131500 \\
\hline C & 4.740300 & -0.206000 & 2.391500 \\
\hline C & 5.563600 & 0.938200 & 2.367000 \\
\hline C & 5.090900 & -1.402700 & 1.685300 \\
\hline C & 6.784600 & 0.972500 & 1.627200 \\
\hline $\mathrm{H}$ & 7.417800 & 1.874100 & 1.631100 \\
\hline C & 6.280700 & -1.365700 & 0.971000 \\
\hline C & 7.116500 & -0.184900 & 0.936100 \\
\hline $\mathrm{H}$ & 5.262700 & 1.823300 & 2.950200 \\
\hline $\mathrm{H}$ & 3.827300 & -0.197600 & 3.007700 \\
\hline $\mathrm{H}$ & 4.449500 & -2.296400 & 1.753700 \\
\hline C & 7.262100 & -2.084300 & 0.090900 \\
\hline C & 8.094300 & -0.905900 & 0.053700 \\
\hline C & 9.290500 & -0.872600 & -0.652500 \\
\hline $\mathrm{H}$ & 9.944300 & 0.013100 & -0.690700 \\
\hline C & 7.607400 & -3.252100 & -0.578000 \\
\hline C & 8.833200 & -3.218200 & -1.305600 \\
\hline C & 9.645500 & -2.069200 & -1.341300 \\
\hline $\mathrm{H}$ & 10.584300 & -2.096800 & -1.917200 \\
\hline $\mathrm{H}$ & 6.990200 & -4.164200 & -0.556300 \\
\hline $\mathrm{H}$ & 9.156500 & -4.118000 & -1.853100 \\
\hline K & -3.880700 & -1.471700 & 0.379700 \\
\hline $\mathrm{H}$ & -7.815700 & -1.099300 & 1.498000 \\
\hline C & -7.289700 & -1.982700 & 1.102500 \\
\hline C & -7.267700 & -2.226700 & -0.285300 \\
\hline C & -6.643900 & -2.871100 & 1.984800 \\
\hline C & -6.599600 & -3.359900 & -0.790000 \\
\hline $\mathrm{H}$ & -7.775700 & -1.533700 & -0.974300 \\
\hline C & -5.974900 & -4.003600 & 1.480200 \\
\hline $\mathrm{H}$ & -6.662800 & -2.681800 & 3.069600 \\
\hline $\mathrm{H}$ & -6.588500 & -3.555300 & -1.874200 \\
\hline C & -5.953500 & -4.248400 & 0.092700 \\
\hline $\mathrm{H}$ & -5.470000 & -4.696900 & 2.170900 \\
\hline $\mathrm{H}$ & -5.436700 & -5.137700 & -0.301400 \\
\hline C & 4.090300 & -0.551000 & -3.336300 \\
\hline C & 3.206400 & 0.486800 & -3.693600 \\
\hline C & 3.489600 & 1.814000 & -3.315900 \\
\hline C & 4.653700 & 2.102000 & -2.576800 \\
\hline C & 5.533800 & 1.063000 & -2.214700 \\
\hline C & 5.253900 & -0.264500 & -2.596400 \\
\hline $\mathrm{H}$ & 3.873500 & -1.586900 & -3.642500 \\
\hline $\mathrm{H}$ & 2.295200 & 0.265100 & -4.270600 \\
\hline $\mathrm{H}$ & 2.799100 & 2.623800 & -3.599600 \\
\hline $\mathrm{H}$ & 4.879300 & 3.141000 & -2.287200 \\
\hline $\mathrm{H}$ & 6.444900 & 1.280800 & -1.636300 \\
\hline $\mathrm{H}$ & & 070 & \\
\hline
\end{tabular}


Table S12. Cartesian coordinates of Int-2

Zero-point correction=

Thermal correction to Energy=

Thermal correction to Enthalpy=

Thermal correction to Gibbs Free Energy=

Sum of electronic and zero-point Energies=

Sum of electronic and thermal Energies=

Sum of electronic and thermal Enthalpies=

Sum of electronic and thermal Free Energies=

\subsection{4 (Hartree/Particle)}

1.416015

1.416960

1.191726

$-5441.529523$

$-5441.441451$

$-5441.440506$

\begin{tabular}{|c|c|c|c|}
\hline $\mathrm{Al}$ & -0.514300 & -0.554600 & 1.053100 \\
\hline $\mathrm{Al}$ & -1.872700 & 1.779100 & -0.838000 \\
\hline C & -1.864800 & 1.820300 & 4.462100 \\
\hline $\mathrm{H}$ & -1.281700 & 2.341900 & 3.676800 \\
\hline $\mathrm{H}$ & -2.721100 & 2.469900 & 4.742700 \\
\hline $\mathrm{H}$ & -1.217200 & 1.722600 & 5.357300 \\
\hline C & -3.999300 & 0.527700 & 2.726300 \\
\hline $\mathrm{H}$ & -4.674900 & -0.347800 & 2.600000 \\
\hline $\mathrm{H}$ & -4.617400 & 1.322600 & 3.195700 \\
\hline $\mathrm{H}$ & -3.651000 & 0.892900 & 1.737800 \\
\hline C & -3.338000 & -0.692300 & 5.381700 \\
\hline $\mathrm{H}$ & -2.636700 & -0.855600 & 6.224100 \\
\hline $\mathrm{H}$ & -4.163300 & -0.045100 & 5.751500 \\
\hline $\mathrm{H}$ & -3.780500 & -1.677300 & 5.120900 \\
\hline C & 1.388500 & 0.474600 & 4.314100 \\
\hline $\mathrm{H}$ & 0.846100 & 1.252900 & 4.886800 \\
\hline $\mathrm{H}$ & 2.339200 & 0.269000 & 4.853000 \\
\hline $\mathrm{H}$ & 1.634300 & 0.897700 & 3.316700 \\
\hline C & 1.578000 & -2.437000 & 3.399000 \\
\hline $\mathrm{H}$ & 1.942900 & -2.123000 & 2.398100 \\
\hline $\mathrm{H}$ & 2.454300 & -2.572200 & 4.069000 \\
\hline $\mathrm{H}$ & 1.076700 & -3.419400 & 3.282900 \\
\hline C & 0.038500 & -1.750200 & 5.911400 \\
\hline $\mathrm{H}$ & -0.613500 & -2.648600 & 5.917200 \\
\hline $\mathrm{H}$ & 0.993800 & -2.026400 & 6.408300 \\
\hline $\mathrm{H}$ & -0.447200 & -0.973000 & 6.537500 \\
\hline $\mathrm{C}$ & -1.188300 & -0.972400 & 3.050400 \\
\hline C & -1.721900 & -2.387300 & 2.886700 \\
\hline $\mathrm{H}$ & -2.182500 & -2.942400 & 3.727300 \\
\hline$C$ & -1.510200 & -3.075700 & 1.715100 \\
\hline $\mathrm{H}$ & -1.788400 & -4.148900 & 1.662200 \\
\hline $\mathrm{C}$ & -0.457300 & -3.353700 & -0.473200 \\
\hline$C$ & 0.081300 & -2.520300 & -1.662300 \\
\hline $\mathrm{H}$ & 0.865600 & -1.827500 & -1.282100 \\
\hline $\mathrm{H}$ & -0.730600 & -1.867300 & -2.052300 \\
\hline C & 0.710700 & -4.250700 & 0.038900 \\
\hline $\mathrm{H}$ & 0.357000 & -4.865300 & 0.895300 \\
\hline $\mathrm{H}$ & 1.507300 & -3.585700 & 0.437800 \\
\hline$C$ & -1.565400 & -4.297000 & -1.046300 \\
\hline $\mathrm{H}$ & -2.397500 & -3.674900 & -1.451900 \\
\hline $\mathrm{H}$ & -1.992900 & -4.924100 & -0.233900 \\
\hline$C$ & 0.640900 & -3.418700 & -2.787200 \\
\hline $\mathrm{H}$ & 1.017300 & -2.773900 & -3.613100 \\
\hline C & 1.794800 & -4.286100 & -2.234800 \\
\hline $\mathrm{H}$ & 2.623300 & -3.637300 & -1.871500 \\
\hline $\mathrm{H}$ & 2.218100 & -4.924300 & -3.042900 \\
\hline$C$ & 1.264800 & -5.161900 & -1.078000 \\
\hline $\mathrm{H}$ & 2.092100 & -5.785500 & -0.671500 \\
\hline C & 0.135900 & -6.077900 & -1.601700 \\
\hline $\mathrm{H}$ & 0.523800 & -6.757700 & -2.392900 \\
\hline $\mathrm{H}$ & -0.241000 & -6.726200 & -0.779400 \\
\hline C & -1.012500 & -5.210500 & -2.165500 \\
\hline
\end{tabular}




\begin{tabular}{|c|c|c|c|}
\hline $\mathrm{H}$ & -1.828400 & -5.869300 & -2.539300 \\
\hline C & -0.478400 & -4.338100 & -3.322900 \\
\hline $\mathrm{H}$ & -0.092300 & -4.981900 & -4.144600 \\
\hline $\mathrm{H}$ & -1.302300 & -3.726700 & -3.753800 \\
\hline C & -4.657300 & 3.750300 & -0.297400 \\
\hline $\mathrm{H}$ & -3.921500 & 4.567800 & -0.442100 \\
\hline $\mathrm{H}$ & -5.640700 & 4.213900 & -0.066800 \\
\hline $\mathrm{H}$ & -4.351600 & 3.165000 & 0.596400 \\
\hline $\mathrm{C}$ & -5.943700 & 1.219900 & -1.440000 \\
\hline $\mathrm{H}$ & -6.136800 & 0.562200 & -2.312600 \\
\hline $\mathrm{H}$ & -5.546100 & 0.599900 & -0.609000 \\
\hline $\mathrm{H}$ & -6.922300 & 1.622000 & -1.100200 \\
\hline C & -5.669200 & 3.716700 & -3.185000 \\
\hline $\mathrm{H}$ & -5.843000 & 3.155300 & -4.125800 \\
\hline $\mathrm{H}$ & -6.657700 & 4.048900 & -2.801200 \\
\hline $\mathrm{H}$ & -5.083200 & 4.625000 & -3.434000 \\
\hline$C$ & -1.078600 & 0.650800 & -4.307900 \\
\hline $\mathrm{H}$ & -0.452300 & 0.336400 & -3.445000 \\
\hline $\mathrm{H}$ & -0.953400 & -0.103600 & -5.113400 \\
\hline $\mathrm{H}$ & -0.688600 & 1.622600 & -4.675100 \\
\hline$C$ & -3.480000 & -0.899900 & -3.192200 \\
\hline $\mathrm{H}$ & -4.505900 & -0.875800 & -2.770200 \\
\hline $\mathrm{H}$ & -3.481200 & -1.621600 & -4.036700 \\
\hline $\mathrm{H}$ & -2.777200 & -1.290900 & -2.427200 \\
\hline$C$ & -3.909800 & 1.242700 & -5.345400 \\
\hline $\mathrm{H}$ & -3.679600 & 2.261800 & -5.719800 \\
\hline $\mathrm{H}$ & -3.677800 & 0.525400 & -6.161800 \\
\hline $\mathrm{H}$ & -5.002400 & 1.191200 & -5.156100 \\
\hline$C$ & -3.030000 & 2.144500 & -2.435500 \\
\hline$C$ & -2.363900 & 3.433400 & -2.907200 \\
\hline $\mathrm{H}$ & -2.639100 & 3.912600 & -3.862400 \\
\hline$C$ & -1.402400 & 4.012000 & -2.125100 \\
\hline $\mathrm{H}$ & -0.908100 & 4.948600 & -2.437100 \\
\hline$C$ & -0.120500 & 4.203300 & -0.023400 \\
\hline$C$ & 1.301900 & 4.366500 & -0.634500 \\
\hline $\mathrm{H}$ & 1.234500 & 4.884700 & -1.616800 \\
\hline $\mathrm{H}$ & 1.717500 & 3.353000 & -0.838700 \\
\hline$C$ & -0.693200 & 5.624500 & 0.265900 \\
\hline $\mathrm{H}$ & -1.708600 & 5.514400 & 0.705900 \\
\hline $\mathrm{H}$ & -0.816400 & 6.190300 & -0.682700 \\
\hline $\mathrm{C}$ & 0.017200 & 3.475200 & 1.331900 \\
\hline $\mathrm{H}$ & -0.994800 & 3.367500 & 1.782900 \\
\hline $\mathrm{H}$ & 0.396100 & 2.437700 & 1.165300 \\
\hline$C$ & 2.228300 & 5.153800 & 0.319700 \\
\hline $\mathrm{H}$ & 3.234900 & 5.257500 & -0.144200 \\
\hline$C$ & 1.629900 & 6.556400 & 0.576000 \\
\hline $\mathrm{H}$ & 2.301600 & 7.142400 & 1.241900 \\
\hline $\mathrm{H}$ & 1.554100 & 7.120300 & -0.380500 \\
\hline $\mathrm{C}$ & 0.233200 & 6.410600 & 1.221800 \\
\hline $\mathrm{H}$ & -0.201000 & 7.419000 & 1.401400 \\
\hline$C$ & 0.359900 & 5.651800 & 2.560900 \\
\hline $\mathrm{H}$ & 1.012900 & 6.216500 & 3.263300 \\
\hline $\mathrm{H}$ & -0.636100 & 5.563500 & 3.047900 \\
\hline C & 0.946100 & 4.247200 & 2.294700 \\
\hline $\mathrm{H}$ & 1.026000 & 3.683100 & 3.249100 \\
\hline$C$ & 2.346700 & 4.393400 & 1.659000 \\
\hline $\mathrm{H}$ & 3.029400 & 4.934900 & 2.351000 \\
\hline $\mathrm{H}$ & 2.798000 & 3.388200 & 1.493500 \\
\hline $\mathrm{N}$ & -0.966400 & -2.461700 & 0.587700 \\
\hline $\mathrm{N}$ & -1.009500 & 3.430700 & -0.911000 \\
\hline 0 & -1.865500 & 0.406100 & 0.183300 \\
\hline Si & -2.504400 & 0.127500 & 3.865300 \\
\hline Si & 0.386400 & -1.141000 & 4.131000 \\
\hline Si & -4.778500 & 2.674900 & -1.863800 \\
\hline Si & -2.901200 & 0.820600 & -3.786000 \\
\hline K & 2.886400 & 0.348300 & -0.029300 \\
\hline C & 4.878000 & -0.163700 & 2.470200 \\
\hline - & 5.620300 & 1.034800 & 2.411300 \\
\hline
\end{tabular}




$\begin{array}{lrrr}\text { C } & 5.262200 & -1.325900 & 1.724700 \\ \mathrm{C} & 6.781000 & 1.162600 & 1.589800 \\ \mathrm{H} & 7.348800 & 2.106500 & 1.564800 \\ \mathrm{C} & 6.397700 & -1.201000 & 0.934500 \\ \mathrm{C} & 7.144600 & 0.039100 & 0.858300 \\ \mathrm{H} & 5.299200 & 1.890800 & 3.026400 \\ \mathrm{H} & 4.005600 & -0.223100 & 3.140000 \\ \mathrm{H} & 4.687000 & -2.261300 & 1.820100 \\ \mathrm{C} & 7.364500 & -1.839400 & -0.016900 \\ \mathrm{C} & 8.108800 & -0.603300 & -0.093600 \\ \mathrm{C} & 9.253700 & -0.482000 & -0.873200 \\ \mathrm{H} & 9.842000 & 0.447000 & -0.940100 \\ \mathrm{C} & 7.749000 & -2.975000 & -0.720500 \\ \mathrm{C} & 8.919500 & -2.849100 & -1.524100 \\ \mathrm{C} & 9.646400 & -1.644800 & -1.597400 \\ \mathrm{H} & 10.547000 & -1.602400 & -2.230700 \\ \mathrm{H} & 7.201700 & -3.929700 & -0.668800 \\ \mathrm{H} & 9.269600 & -3.720000 & -2.101200 \\ \mathrm{~K} & -3.710100 & -1.585700 & 0.338400 \\ \mathrm{H} & -7.617900 & -1.420300 & 1.486500 \\ \mathrm{C} & -7.056400 & -2.279700 & 1.087100 \\ \mathrm{C} & -7.045300 & -2.530600 & -0.300000 \\ \mathrm{C} & -6.356400 & -3.132300 & 1.963200 \\ \mathrm{C} & -6.333300 & -3.634400 & -0.809500 \\ \mathrm{H} & -7.597400 & -1.866500 & -0.983400 \\ \mathrm{C} & -5.643400 & -4.235500 & 1.453800 \\ \mathrm{H} & -6.367300 & -2.938300 & 3.047300 \\ \mathrm{H} & -6.330500 & -3.836000 & -1.892500 \\ \mathrm{C} & -5.632900 & -4.486900 & 0.067200 \\ \mathrm{H} & -5.095500 & -4.900100 & 2.139800 \\ \mathrm{H} & -5.081200 & -5.353100 & -0.330700 \\ \mathrm{C} & 3.776600 & -0.441800 & -3.379600 \\ \mathrm{C} & 3.005800 & 0.719400 & -3.589300 \\ \mathrm{C} & 3.509700 & 1.977300 & -3.203600 \\ \mathrm{C} & 4.778300 & 2.071800 & -2.599000 \\ \mathrm{C} & 5.545900 & 0.910200 & -2.383400 \\ \mathrm{C} & 5.046700 & -0.347500 & -2.778200 \\ \mathrm{H} & 3.386900 & -1.423100 & -3.694100 \\ \mathrm{H} & 2.013700 & 0.646400 & -4.062100 \\ \mathrm{H} & 2.910600 & 2.884900 & -3.377900 \\ \mathrm{H} & 5.174900 & 3.055700 & -2.300800 \\ \mathrm{H} & 6.539400 & 0.975200 & -1.913100 \\ \mathrm{H} & 5.657300 & -1.249600 & -2.614100\end{array}$

Table S13. Cartesian coordinates of TS-2

Zero-point correction=

Thermal correction to Energy=

1.326882 (Hartree/Particle)

Thermal correction to Enthalpy=

1.413891

Thermal correction to Gibbs Free Energy= 1.196822

Sum of electronic and zero-point Energies= -5441.510774

Sum of electronic and thermal Energies $=\quad-5441.423765$

Sum of electronic and thermal Enthalpies $=-5441.422821$

Sum of electronic and thermal Free Energies $=-5441.640833$

$\begin{array}{lrrr}\text { Al } & -0.436600 & -0.882600 & 1.251600 \\ \text { Al } & -0.888200 & 2.022200 & -0.567500 \\ \text { C } & -1.533100 & 1.487500 & 4.293100 \\ \text { H } & -0.910100 & 1.909400 & 3.479200 \\ \text { H } & -2.140200 & 2.312600 & 4.723600 \\ \text { H } & -0.848300 & 1.121500 & 5.083000 \\ \text { C } & -3.828100 & 0.966000 & 2.368500 \\ \text { H } & -4.665000 & 0.305300 & 2.050900\end{array}$




\begin{tabular}{|c|c|c|c|}
\hline $\mathrm{H}$ & -4.294300 & 1.859700 & 2.835200 \\
\hline $\mathrm{H}$ & -3.248600 & 1.311300 & 1.486600 \\
\hline C & -3.854300 & -0.323100 & 5.076200 \\
\hline $\mathrm{H}$ & -3.306500 & -0.562100 & 6.010600 \\
\hline $\mathrm{H}$ & -4.507500 & 0.550700 & 5.290800 \\
\hline $\mathrm{H}$ & -4.513200 & -1.185800 & 4.843000 \\
\hline C & 0.232800 & -1.436500 & 5.459200 \\
\hline $\mathrm{H}$ & -0.372000 & -0.809700 & 6.147800 \\
\hline $\mathrm{H}$ & 0.819700 & -2.141300 & 6.086500 \\
\hline $\mathrm{H}$ & 0.956100 & -0.774300 & 4.941400 \\
\hline C & 0.116800 & -3.876000 & 3.541600 \\
\hline $\mathrm{H}$ & 1.028300 & -3.580600 & 2.990000 \\
\hline $\mathrm{H}$ & 0.435600 & -4.537700 & 4.375700 \\
\hline $\mathrm{H}$ & -0.522000 & -4.474800 & 2.861600 \\
\hline C & -2.151100 & -3.325400 & 5.378400 \\
\hline $\mathrm{H}$ & -2.832500 & -3.970700 & 4.785700 \\
\hline $\mathrm{H}$ & -1.592900 & -3.990000 & 6.073200 \\
\hline $\mathrm{H}$ & -2.772700 & -2.647900 & 5.995000 \\
\hline C & -1.721600 & -1.353600 & 2.887300 \\
\hline C & -2.619800 & -2.393100 & 2.226100 \\
\hline $\mathrm{H}$ & -3.498600 & -2.822400 & 2.743800 \\
\hline C & -2.225600 & -2.975400 & 1.042900 \\
\hline $\mathrm{H}$ & -2.789000 & -3.844200 & 0.643600 \\
\hline C & -0.638700 & -3.289500 & -0.805100 \\
\hline C & 0.557500 & -2.561900 & -1.467800 \\
\hline $\mathrm{H}$ & 1.339300 & -2.405700 & -0.690600 \\
\hline $\mathrm{H}$ & 0.216800 & -1.556200 & -1.808400 \\
\hline C & -0.140500 & -4.692400 & -0.340300 \\
\hline $\mathrm{H}$ & -0.981000 & -5.243900 & 0.135200 \\
\hline $\mathrm{H}$ & 0.627700 & -4.551900 & 0.449600 \\
\hline C & -1.715600 & -3.514600 & -1.913100 \\
\hline $\mathrm{H}$ & -2.062500 & -2.522200 & -2.282600 \\
\hline $\mathrm{H}$ & -2.600700 & -4.034300 & -1.484000 \\
\hline C & 1.132000 & -3.372200 & -2.653100 \\
\hline $\mathrm{H}$ & 1.988000 & -2.814600 & -3.094100 \\
\hline C & 1.617700 & -4.749000 & -2.147400 \\
\hline $\mathrm{H}$ & 2.427900 & -4.615600 & -1.397700 \\
\hline $\mathrm{H}$ & 2.051500 & -5.334500 & -2.988300 \\
\hline C & 0.428600 & -5.510500 & -1.521800 \\
\hline $\mathrm{H}$ & 0.773200 & -6.500700 & -1.150500 \\
\hline C & -0.672300 & -5.711000 & -2.587200 \\
\hline $\mathrm{H}$ & -0.282200 & -6.316700 & -3.435200 \\
\hline $\mathrm{H}$ & -1.526300 & -6.278700 & -2.154500 \\
\hline C & -1.152800 & -4.333700 & -3.098200 \\
\hline $\mathrm{H}$ & -1.950800 & -4.474300 & -3.861400 \\
\hline C & 0.036000 & -3.570800 & -3.723100 \\
\hline $\mathrm{H}$ & 0.442400 & -4.136700 & -4.590600 \\
\hline $\mathrm{H}$ & -0.303600 & -2.585300 & -4.113200 \\
\hline C & -3.139400 & 4.563300 & 0.045800 \\
\hline $\mathrm{H}$ & -2.258500 & 5.235300 & 0.003200 \\
\hline $\mathrm{H}$ & -4.024800 & 5.178900 & 0.313700 \\
\hline $\mathrm{H}$ & -2.982100 & 3.838000 & 0.873100 \\
\hline C & -4.917000 & 2.556500 & -1.415000 \\
\hline $\mathrm{H}$ & -5.151900 & 1.968200 & -2.325700 \\
\hline $\mathrm{H}$ & -4.787600 & 1.864000 & -0.556600 \\
\hline $\mathrm{H}$ & -5.811400 & 3.173500 & -1.182000 \\
\hline C & -3.925400 & 5.068300 & -2.861500 \\
\hline $\mathrm{H}$ & -4.200900 & 4.667000 & -3.857500 \\
\hline $\mathrm{H}$ & -4.806000 & 5.615600 & -2.462200 \\
\hline $\mathrm{H}$ & -3.110000 & 5.806300 & -3.004800 \\
\hline C & -0.127900 & 1.125700 & -4.169900 \\
\hline $\mathrm{H}$ & 0.262100 & 0.418700 & -3.406100 \\
\hline $\mathrm{H}$ & -0.116800 & 0.598100 & -5.147200 \\
\hline $\mathrm{H}$ & 0.572000 & 1.984200 & -4.231100 \\
\hline C & -2.951700 & 0.151000 & -3.552800 \\
\hline $\mathrm{H}$ & -3.973200 & 0.356300 & -3.170700 \\
\hline $\mathrm{H}$ & -3.068500 & -0.330100 & -4.547400 \\
\hline $\mathrm{H}$ & -2.449700 & -0.596900 & -2.903600 \\
\hline
\end{tabular}




\begin{tabular}{|c|c|c|c|}
\hline C & -2.560600 & 2.656700 & -5.245600 \\
\hline $\mathrm{H}$ & -2.056800 & 3.633500 & -5.397100 \\
\hline $\mathrm{H}$ & -2.403300 & 2.052900 & -6.165000 \\
\hline $\mathrm{H}$ & -3.650100 & 2.848800 & -5.157000 \\
\hline C & -1.801600 & 2.813500 & -2.178100 \\
\hline C & -0.803500 & 3.934000 & -2.458500 \\
\hline $\mathrm{H}$ & -0.884900 & 4.563800 & -3.361100 \\
\hline C & 0.221200 & 4.165200 & -1.585900 \\
\hline $\mathrm{H}$ & 0.943100 & 4.979800 & -1.770400 \\
\hline C & 1.231200 & 3.953300 & 0.644200 \\
\hline C & 2.704700 & 4.176500 & 0.196700 \\
\hline $\mathrm{H}$ & 2.743500 & 4.846000 & -0.690000 \\
\hline $\mathrm{H}$ & 3.142500 & 3.204500 & -0.125100 \\
\hline C & 0.651700 & 5.326400 & 1.104400 \\
\hline $\mathrm{H}$ & -0.398900 & 5.172800 & 1.433300 \\
\hline $\mathrm{H}$ & 0.617400 & 6.026600 & 0.241700 \\
\hline C & 1.235800 & 3.016400 & 1.870100 \\
\hline $\mathrm{H}$ & 0.186300 & 2.856100 & 2.201200 \\
\hline $\mathrm{H}$ & 1.632900 & 2.011500 & 1.599900 \\
\hline C & 3.544000 & 4.782200 & 1.346600 \\
\hline $\mathrm{H}$ & 4.589800 & 4.926100 & 0.995700 \\
\hline C & 2.942700 & 6.143400 & 1.761800 \\
\hline $\mathrm{H}$ & 3.557800 & 6.600700 & 2.567800 \\
\hline $\mathrm{H}$ & 2.962200 & 6.852200 & 0.903600 \\
\hline C & 1.491000 & 5.936100 & 2.248400 \\
\hline $\mathrm{H}$ & 1.050300 & 6.914600 & 2.541400 \\
\hline C & 1.475100 & 4.972300 & 3.455000 \\
\hline $\mathrm{H}$ & 2.062100 & 5.397800 & 4.298800 \\
\hline $\mathrm{H}$ & 0.434500 & 4.836700 & 3.823500 \\
\hline C & 2.071000 & 3.613700 & 3.025900 \\
\hline $\mathrm{H}$ & 2.050900 & 2.907600 & 3.883800 \\
\hline C & 3.526800 & 3.817800 & 2.552000 \\
\hline $\mathrm{H}$ & 4.147100 & 4.225800 & 3.380200 \\
\hline $\mathrm{H}$ & 3.971200 & 2.840200 & 2.266300 \\
\hline $\mathrm{N}$ & -1.148800 & -2.479000 & 0.315100 \\
\hline $\mathrm{N}$ & 0.397400 & 3.375400 & -0.436300 \\
\hline 0 & -1.157000 & 0.548000 & 0.266300 \\
\hline $\mathrm{Si}$ & -2.669200 & 0.111800 & 3.637900 \\
\hline $\mathrm{Si}$ & -0.882900 & -2.424700 & 4.264000 \\
\hline $\mathrm{Si}$ & -3.410500 & 3.711000 & -1.632800 \\
\hline $\mathrm{Si}$ & -1.880000 & 1.721900 & -3.732700 \\
\hline K & 2.815800 & 0.193000 & -0.509700 \\
\hline C & 2.271600 & -1.248800 & 2.248300 \\
\hline C & 3.121600 & -0.141800 & 2.654000 \\
\hline C & 2.874900 & -2.326500 & 1.463900 \\
\hline C & 4.442700 & 0.023700 & 2.187000 \\
\hline $\mathrm{H}$ & 5.056800 & 0.859100 & 2.565500 \\
\hline C & 4.149300 & -2.137800 & 0.985100 \\
\hline C & 4.950700 & -0.936100 & 1.287000 \\
\hline $\mathrm{H}$ & 2.725200 & 0.571500 & 3.394300 \\
\hline $\mathrm{H}$ & 1.478100 & -1.535400 & 2.949100 \\
\hline $\mathrm{H}$ & 2.317500 & -3.263700 & 1.301900 \\
\hline C & 5.321400 & -2.716100 & 0.256500 \\
\hline C & 6.102000 & -1.534400 & 0.591000 \\
\hline C & 7.449900 & -1.436200 & 0.208400 \\
\hline $\mathrm{H}$ & 8.090200 & -0.579200 & 0.474700 \\
\hline C & 5.844200 & -3.774300 & -0.473200 \\
\hline C & 7.205300 & -3.652700 & -0.888600 \\
\hline C & 7.974500 & -2.519000 & -0.546600 \\
\hline $\mathrm{H}$ & 9.027500 & -2.477600 & -0.872400 \\
\hline $\mathrm{H}$ & 5.260500 & -4.676300 & -0.720700 \\
\hline $\mathrm{H}$ & 7.670300 & -4.464800 & -1.469700 \\
\hline K & -3.572500 & -0.763100 & -0.330200 \\
\hline $\mathrm{H}$ & -7.566600 & 0.228900 & -0.096000 \\
\hline C & -7.118700 & -0.738400 & -0.373600 \\
\hline C & -6.867000 & -1.034600 & -1.729000 \\
\hline C & -6.809500 & -1.682700 & 0.624200 \\
\hline C & -6.304700 & -2.278200 & -2.083800 \\
\hline
\end{tabular}




$\begin{array}{rrrr}\text { H } & -7.120900 & -0.300000 & -2.509400 \\ \mathrm{C} & -6.246400 & -2.925900 & 0.269900 \\ \mathrm{H} & -7.014100 & -1.454200 & 1.682100 \\ \mathrm{H} & -6.118000 & -2.516400 & -3.143100 \\ \mathrm{C} & -5.996100 & -3.222200 & -1.085300 \\ \mathrm{H} & -6.008200 & -3.665300 & 1.049900 \\ \mathrm{H} & -5.566500 & -4.197300 & -1.364500 \\ \mathrm{C} & 3.670400 & 0.566500 & -4.051700 \\ \mathrm{C} & 4.027300 & 1.816700 & -3.508200 \\ \mathrm{C} & 5.063100 & 1.900200 & -2.556700 \\ \mathrm{C} & 5.734100 & 0.732000 & -2.139800 \\ \mathrm{C} & 5.386200 & -0.518600 & -2.691100 \\ \mathrm{C} & 4.356200 & -0.598500 & -3.647400 \\ \mathrm{H} & 2.865600 & 0.502800 & -4.800700 \\ \mathrm{H} & 3.501200 & 2.728700 & -3.832800 \\ \mathrm{H} & 5.350400 & 2.879100 & -2.140400 \\ \mathrm{H} & 6.541200 & 0.781800 & -1.393200 \\ \mathrm{H} & 5.918400 & -1.424800 & -2.360200 \\ \mathrm{H} & 4.090700 & -1.573900 & -4.085600\end{array}$

Table S14. Cartesian coordinates of Int-3

$\begin{array}{lr}\text { Zero-point correction= } & 1.325876 \text { (Hartree/Particle) } \\ \text { Thermal correction to Energy= } & 1.413282 \\ \text { Thermal correction to Enthalpy= } & 1.414226 \\ \text { Thermal correction to Gibbs Free Energy= } & 1.194320 \\ \text { Sum of electronic and zero-point Energies= } & -5441.531182 \\ \text { Sum of electronic and thermal Energies= } & -5441.443777 \\ \text { Sum of electronic and thermal Enthalpies= } & -5441.442832 \\ \text { Sum of electronic and thermal Free Energies= } & -5441.662738\end{array}$

$\begin{array}{lrrr}\text { Al } & -0.641000 & -0.975900 & 1.291800 \\ \text { Al } & -0.174500 & 2.014400 & -0.570000 \\ \text { C } & -0.808300 & 1.720000 & 4.303000 \\ \text { H } & 0.033700 & 1.698300 & 3.583000 \\ \text { H } & -1.031700 & 2.781600 & 4.542500 \\ \text { H } & -0.458900 & 1.231200 & 5.233900 \\ \text { C } & -3.070500 & 2.034600 & 2.268500 \\ \text { H } & -4.089100 & 1.721100 & 1.947700 \\ \text { H } & -3.183100 & 3.052400 & 2.698900 \\ \text { H } & -2.400900 & 2.122700 & 1.386200 \\ \text { C } & -3.685700 & 0.918800 & 4.965200 \\ \text { H } & -3.306800 & 0.559800 & 5.943300 \\ \text { H } & -4.022200 & 1.968800 & 5.107100 \\ \text { H } & -4.579300 & 0.311500 & 4.709100 \\ \text { C } & -0.012200 & -1.605600 & 5.399500 \\ \text { H } & -0.278200 & -0.773800 & 6.084600 \\ \text { H } & 0.283600 & -2.468100 & 6.035000 \\ \text { H } & 0.881300 & -1.300300 & 4.817600 \\ \text { C } & -1.141100 & -3.871900 & 3.626900 \\ \text { H } & -0.219600 & -3.950100 & 3.017200 \\ \text { H } & -1.028900 & -4.567200 & 4.486700 \\ \text { H } & -1.994000 & -4.227700 & 3.014100 \\ \text { C } & -2.937700 & -2.427700 & 5.529000 \\ \text { H } & -3.875200 & -2.707800 & 5.005400 \\ \text { H } & -2.666400 & -3.276500 & 6.193700 \\ \text { H } & -3.155500 & -1.557600 & 6.179100 \\ \text { C } & -1.967800 & -0.870900 & 2.908500 \\ \text { C } & -3.188800 & -1.557900 & 2.307100 \\ \text { H } & -4.148800 & -1.632900 & 2.851400 \\ \text { C } & -3.051600 & -2.273500 & 1.140600 \\ \text { H } & -3.887600 & -2.903400 & 0.778100 \\ \text { C } & -1.712000 & -3.165300 & -0.725000\end{array}$




\begin{tabular}{|c|c|c|c|}
\hline C & -0.493800 & -2.741700 & -1.583100 \\
\hline $\mathrm{H}$ & 0.399200 & -2.659900 & -0.925800 \\
\hline $\mathrm{H}$ & -0.691700 & -1.729500 & -2.002100 \\
\hline C & -1.458300 & -4.602400 & -0.176200 \\
\hline $\mathrm{H}$ & -2.340300 & -4.922400 & 0.421700 \\
\hline $\mathrm{H}$ & -0.600600 & -4.574500 & 0.527300 \\
\hline C & -2.938100 & -3.256700 & -1.689900 \\
\hline $\mathrm{H}$ & -3.149000 & -2.249100 & -2.118900 \\
\hline $\mathrm{H}$ & -3.847900 & -3.567100 & -1.131800 \\
\hline C & -0.219600 & -3.747700 & -2.723600 \\
\hline $\mathrm{H}$ & 0.671600 & -3.413400 & -3.300100 \\
\hline C & 0.046600 & -5.147800 & -2.124900 \\
\hline $\mathrm{H}$ & 0.942700 & -5.117500 & -1.467900 \\
\hline $\mathrm{H}$ & 0.268100 & -5.875000 & -2.937400 \\
\hline C & -1.190100 & -5.606500 & -1.319300 \\
\hline $\mathrm{H}$ & -0.999900 & -6.613300 & -0.886600 \\
\hline C & -2.421500 & -5.665000 & -2.249500 \\
\hline $\mathrm{H}$ & -2.254100 & -6.397700 & -3.070000 \\
\hline $\mathrm{H}$ & -3.313700 & -6.015100 & -1.683800 \\
\hline C & -2.680800 & -4.260900 & -2.840100 \\
\hline $\mathrm{H}$ & -3.575800 & -4.292300 & -3.501100 \\
\hline C & -1.449000 & -3.811000 & -3.655800 \\
\hline $\mathrm{H}$ & -1.265100 & -4.520500 & -4.492900 \\
\hline $\mathrm{H}$ & -1.635700 & -2.814400 & -4.115000 \\
\hline C & -1.845000 & 5.236500 & -0.443000 \\
\hline $\mathrm{H}$ & -0.882100 & 5.752800 & -0.629700 \\
\hline $\mathrm{H}$ & -2.624700 & 6.012800 & -0.288400 \\
\hline $\mathrm{H}$ & -1.752700 & 4.668500 & 0.507000 \\
\hline C & -4.007400 & 3.351800 & -1.447400 \\
\hline $\mathrm{H}$ & -4.352000 & 2.569000 & -2.154300 \\
\hline $\mathrm{H}$ & -4.014700 & 2.953700 & -0.410400 \\
\hline $\mathrm{H}$ & -4.774900 & 4.155200 & -1.467600 \\
\hline C & -2.631600 & 5.247100 & -3.405200 \\
\hline $\mathrm{H}$ & -3.111200 & 4.709500 & -4.248700 \\
\hline $\mathrm{H}$ & -3.309700 & 6.075500 & -3.108100 \\
\hline $\mathrm{H}$ & -1.693300 & 5.703500 & -3.780800 \\
\hline C & 0.472800 & 0.563400 & -3.783800 \\
\hline $\mathrm{H}$ & 0.501000 & -0.161600 & -2.941800 \\
\hline $\mathrm{H}$ & 0.516700 & -0.029400 & -4.721600 \\
\hline $\mathrm{H}$ & 1.380000 & 1.202200 & -3.743000 \\
\hline C & -2.585300 & 0.451700 & -3.578600 \\
\hline $\mathrm{H}$ & -3.547100 & 0.972200 & -3.388500 \\
\hline $\mathrm{H}$ & -2.701800 & -0.097800 & -4.536800 \\
\hline $\mathrm{H}$ & -2.414600 & -0.322100 & -2.801900 \\
\hline C & -1.261100 & 2.520600 & -5.384400 \\
\hline $\mathrm{H}$ & -0.453500 & 3.262100 & -5.551500 \\
\hline $\mathrm{H}$ & -1.197100 & 1.770000 & -6.201300 \\
\hline $\mathrm{H}$ & -2.231500 & 3.047000 & -5.491800 \\
\hline C & -0.918800 & 2.849800 & -2.245600 \\
\hline C & 0.291500 & 3.720900 & -2.584300 \\
\hline $\mathrm{H}$ & 0.343500 & 4.280900 & -3.533100 \\
\hline C & 1.330600 & 3.822600 & -1.704700 \\
\hline $\mathrm{H}$ & 2.200700 & 4.462000 & -1.933000 \\
\hline C & 2.238800 & 3.628300 & 0.569400 \\
\hline C & 3.734800 & 3.457200 & 0.181000 \\
\hline $\mathrm{H}$ & 3.951000 & 3.995500 & -0.768100 \\
\hline $\mathrm{H}$ & 3.942300 & 2.380200 & -0.008200 \\
\hline C & 1.975500 & 5.138600 & 0.850000 \\
\hline $\mathrm{H}$ & 0.907400 & 5.265400 & 1.131500 \\
\hline $\mathrm{H}$ & 2.134600 & 5.734400 & -0.075000 \\
\hline C & 1.984800 & 2.860100 & 1.879500 \\
\hline $\mathrm{H}$ & 0.918700 & 2.999000 & 2.169500 \\
\hline $\mathrm{H}$ & 2.139600 & 1.768800 & 1.723000 \\
\hline C & 4.657800 & 3.980500 & 1.305900 \\
\hline $\mathrm{H}$ & 5.718300 & 3.845700 & 0.998400 \\
\hline C & 4.372900 & 5.479700 & 1.549100 \\
\hline $\mathrm{H}$ & 5.050900 & 5.872700 & 2.338300 \\
\hline $\mathrm{H}$ & 4.580900 & 6.066500 & 0.626200 \\
\hline
\end{tabular}




\begin{tabular}{|c|c|c|c|}
\hline C & 2.898200 & 5.660000 & 1.974600 \\
\hline $\mathrm{H}$ & 2.687200 & 6.738900 & 2.145000 \\
\hline C & 2.630500 & 4.863900 & 3.271200 \\
\hline $\mathrm{H}$ & 3.278700 & 5.239300 & 4.093800 \\
\hline $\mathrm{H}$ & 1.577700 & 5.010200 & 3.600000 \\
\hline C & 2.906600 & 3.366400 & 3.012900 \\
\hline $\mathrm{H}$ & 2.699300 & 2.779100 & 3.933300 \\
\hline C & 4.384300 & 3.182000 & 2.599000 \\
\hline $\mathrm{H}$ & 5.056600 & 3.529300 & 3.414200 \\
\hline $\mathrm{H}$ & 4.604100 & 2.104900 & 2.437000 \\
\hline $\mathrm{N}$ & -1.894600 & -2.185100 & 0.366000 \\
\hline $\mathrm{N}$ & 1.331900 & 3.119300 & -0.489400 \\
\hline 0 & -0.576100 & 0.561500 & 0.247600 \\
\hline $\mathrm{Si}$ & -2.345700 & 0.851800 & 3.602800 \\
\hline Si & -1.490800 & -2.125300 & 4.313300 \\
\hline $\mathrm{Si}$ & -2.318000 & 4.120100 & -1.905800 \\
\hline $\mathrm{Si}$ & -1.097500 & 1.638800 & -3.706800 \\
\hline K & 2.831300 & -0.373300 & -0.736500 \\
\hline C & 1.216800 & -1.722400 & 1.834800 \\
\hline C & 2.225700 & -0.706900 & 2.371000 \\
\hline C & 1.857500 & -2.855400 & 1.046500 \\
\hline C & 3.612100 & -0.815900 & 2.223600 \\
\hline $\mathrm{H}$ & 4.263300 & -0.086800 & 2.739500 \\
\hline C & 3.207100 & -2.875700 & 0.869400 \\
\hline C & 4.143300 & -1.855200 & 1.417000 \\
\hline $\mathrm{H}$ & 1.831100 & 0.070000 & 3.046300 \\
\hline $\mathrm{H}$ & 0.743600 & -2.211700 & 2.728200 \\
\hline $\mathrm{H}$ & 1.226200 & -3.699600 & 0.723500 \\
\hline C & 4.382400 & -3.632600 & 0.339000 \\
\hline C & 5.270400 & -2.634300 & 0.929400 \\
\hline C & 6.672500 & -2.795600 & 0.828900 \\
\hline $\mathrm{H}$ & 7.392200 & -2.101400 & 1.293200 \\
\hline C & 4.843700 & -4.737500 & -0.360700 \\
\hline C & 6.260900 & -4.869200 & -0.490000 \\
\hline C & 7.129900 & -3.921900 & 0.101000 \\
\hline $\mathrm{H}$ & 8.218100 & -4.073900 & -0.004300 \\
\hline $\mathrm{H}$ & 4.166700 & -5.491400 & -0.797000 \\
\hline $\mathrm{H}$ & 6.684200 & -5.727700 & -1.035000 \\
\hline K & -3.583500 & 0.166200 & -0.337200 \\
\hline $\mathrm{H}$ & -7.172000 & 2.321000 & -0.447600 \\
\hline C & -7.020200 & 1.234500 & -0.547400 \\
\hline C & -6.884400 & 0.659100 & -1.826000 \\
\hline C & -6.973200 & 0.422700 & 0.603000 \\
\hline C & -6.703500 & -0.735500 & -1.955100 \\
\hline $\mathrm{H}$ & -6.936300 & 1.294300 & -2.724500 \\
\hline C & -6.794100 & -0.972400 & 0.474300 \\
\hline $\mathrm{H}$ & -7.089600 & 0.872700 & 1.601700 \\
\hline $\mathrm{H}$ & -6.611500 & -1.190200 & -2.954200 \\
\hline C & -6.662000 & -1.547600 & -0.804200 \\
\hline $\mathrm{H}$ & -6.763800 & -1.608900 & 1.372000 \\
\hline $\mathrm{H}$ & -6.534200 & -2.637000 & -0.905600 \\
\hline C & 4.280700 & -0.233300 & -4.118300 \\
\hline C & 4.801100 & 0.918000 & -3.492400 \\
\hline C & 5.632200 & 0.787900 & -2.361900 \\
\hline C & 5.938600 & -0.490600 & -1.851600 \\
\hline C & 5.424000 & -1.644600 & -2.481100 \\
\hline C & 4.594200 & -1.511800 & -3.611300 \\
\hline $\mathrm{H}$ & 3.639700 & -0.134200 & -5.008700 \\
\hline $\mathrm{H}$ & 4.567100 & 1.917400 & -3.893400 \\
\hline $\mathrm{H}$ & 6.050600 & 1.687100 & -1.881400 \\
\hline $\mathrm{H}$ & 6.583800 & -0.610000 & -0.967400 \\
\hline $\mathrm{H}$ & 5.672000 & -2.639600 & -2.077000 \\
\hline 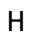 & 4.198200 & -2.413000 & -4.10690 \\
\hline
\end{tabular}

Table S15. Cartesian coordinates of Int-4 
Zero-point correction= Thermal correction to Energy= Thermal correction to Enthalpy= Thermal correction to Gibbs Free Energy= Sum of electronic and zero-point Energies= Sum of electronic and thermal Energies= Sum of electronic and thermal Enthalpies= Sum of electronic and thermal Free Energies $=-5441.690672$
1.329399 (Hartree/Particle)

1.415096

1.416040

1.200606

$-5441.561879$

$-5441.476182$

$-5441.475238$

\begin{tabular}{|c|c|c|c|}
\hline Al & -1.669100 & -0.238400 & 1.214500 \\
\hline $\mathrm{Al}$ & 0.743200 & 0.609500 & -0.463200 \\
\hline C & -1.704200 & 3.574200 & 3.389700 \\
\hline $\mathrm{H}$ & -0.707800 & 3.105700 & 3.262100 \\
\hline $\mathrm{H}$ & -1.621300 & 4.646500 & 3.112200 \\
\hline $\mathrm{H}$ & -1.959100 & 3.531100 & 4.467700 \\
\hline C & -2.835300 & 3.400300 & 0.541200 \\
\hline $\mathrm{H}$ & -3.778700 & 3.299000 & -0.041300 \\
\hline $\mathrm{H}$ & -2.607400 & 4.487200 & 0.562900 \\
\hline $\mathrm{H}$ & -2.003000 & 2.875400 & 0.025500 \\
\hline$C$ & -4.704800 & 3.511200 & 2.888600 \\
\hline $\mathrm{H}$ & -4.940000 & 3.309600 & 3.953100 \\
\hline $\mathrm{H}$ & -4.662500 & 4.614600 & 2.757300 \\
\hline $\mathrm{H}$ & -5.558600 & 3.138700 & 2.283600 \\
\hline C & -1.508600 & 0.719400 & 5.285100 \\
\hline $\mathrm{H}$ & -1.596500 & 1.765900 & 5.639100 \\
\hline $\mathrm{H}$ & -1.464400 & 0.068200 & 6.185100 \\
\hline $\mathrm{H}$ & -0.535800 & 0.620500 & 4.761000 \\
\hline C & -3.024000 & -1.722400 & 4.256300 \\
\hline $\mathrm{H}$ & -2.066100 & -2.168000 & 3.919600 \\
\hline $\mathrm{H}$ & -3.202600 & -2.064900 & 5.298400 \\
\hline $\mathrm{H}$ & -3.837700 & -2.123300 & 3.620200 \\
\hline C & -4.545100 & 0.717400 & 5.206700 \\
\hline $\mathrm{H}$ & -5.485600 & 0.510900 & 4.655000 \\
\hline $\mathrm{H}$ & -4.587100 & 0.154100 & 6.164000 \\
\hline $\mathrm{H}$ & -4.527100 & 1.797500 & 5.461100 \\
\hline C & -3.027100 & 0.842500 & 2.403200 \\
\hline $\mathrm{C}$ & -4.336200 & 0.306400 & 1.832000 \\
\hline $\mathrm{H}$ & -5.318500 & 0.728200 & 2.114000 \\
\hline$C$ & -4.325300 & -0.824300 & 1.050300 \\
\hline $\mathrm{H}$ & -5.285500 & -1.280200 & 0.736600 \\
\hline C & -3.224900 & -2.776800 & 0.075200 \\
\hline C & -1.825200 & -3.262200 & -0.366800 \\
\hline $\mathrm{H}$ & -1.132200 & -3.221800 & 0.499900 \\
\hline $\mathrm{H}$ & -1.418600 & -2.554200 & -1.122000 \\
\hline C & -3.764900 & -3.791200 & 1.130400 \\
\hline $\mathrm{H}$ & -4.771500 & -3.466900 & 1.474400 \\
\hline $\mathrm{H}$ & -3.101600 & -3.759500 & 2.020600 \\
\hline C & -4.159100 & -2.849600 & -1.175300 \\
\hline $\mathrm{H}$ & -3.769900 & -2.156600 & -1.957100 \\
\hline $\mathrm{H}$ & -5.182600 & -2.505700 & -0.909600 \\
\hline C & -1.881100 & -4.697700 & -0.941300 \\
\hline $\mathrm{H}$ & -0.856600 & -5.006300 & -1.243900 \\
\hline C & -2.417900 & -5.668800 & 0.134100 \\
\hline $\mathrm{H}$ & -1.739900 & -5.677300 & 1.015900 \\
\hline $\mathrm{H}$ & -2.440300 & -6.707800 & -0.264800 \\
\hline $\mathrm{C}$ & -3.834900 & -5.224400 & 0.559200 \\
\hline $\mathrm{H}$ & -4.224600 & -5.913600 & 1.341100 \\
\hline C & -4.777200 & -5.244400 & -0.665100 \\
\hline $\mathrm{H}$ & -4.857100 & -6.275600 & -1.076100 \\
\hline $\mathrm{H}$ & -5.803700 & -4.939100 & -0.361900 \\
\hline C & -4.232600 & -4.284800 & -1.746900 \\
\hline $\mathrm{H}$ & -4.909300 & -4.294900 & -2.631100 \\
\hline C & -2.818000 & -4.735800 & -2.169500 \\
\hline $\mathrm{H}$ & -2.852400 & -5.763700 & -2.595100 \\
\hline $\mathrm{H}$ & -2.429200 & -4.071800 & -2.973000 \\
\hline C & -0.363300 & 2.261800 & -3.367500 \\
\hline
\end{tabular}




\begin{tabular}{|c|c|c|c|}
\hline $\mathrm{H}$ & 0.534800 & 2.909700 & -3.404300 \\
\hline $\mathrm{H}$ & -1.049400 & 2.582700 & -4.182000 \\
\hline $\mathrm{H}$ & -0.857900 & 2.436900 & -2.390100 \\
\hline C & -1.551700 & -0.539800 & -3.629600 \\
\hline $\mathrm{H}$ & -1.772500 & -0.948800 & -2.622600 \\
\hline $\mathrm{H}$ & -2.382500 & 0.124200 & -3.958800 \\
\hline $\mathrm{H}$ & -1.531900 & -1.393700 & -4.334600 \\
\hline C & 0.765400 & 0.341600 & -5.413200 \\
\hline $\mathrm{H}$ & 0.989400 & -0.696100 & -5.737200 \\
\hline $\mathrm{H}$ & 0.006800 & 0.750600 & -6.115400 \\
\hline $\mathrm{H}$ & 1.689900 & 0.943500 & -5.533400 \\
\hline C & 2.504100 & -2.992800 & -1.174800 \\
\hline $\mathrm{H}$ & 2.029200 & -2.693900 & -0.216700 \\
\hline H & 2.250900 & -4.062900 & -1.330600 \\
\hline $\mathrm{H}$ & 3.609100 & -2.988400 & -1.042500 \\
\hline C & 0.553700 & -3.086200 & -3.494400 \\
\hline $\mathrm{H}$ & 0.280000 & -2.739800 & -4.511700 \\
\hline $\mathrm{H}$ & 0.956100 & -4.116900 & -3.591500 \\
\hline $\mathrm{H}$ & -0.371900 & -3.142700 & -2.890800 \\
\hline C & 3.351200 & -2.068900 & -3.935500 \\
\hline $\mathrm{H}$ & 4.286200 & -1.614400 & -3.542300 \\
\hline $\mathrm{H}$ & 3.576000 & -3.131700 & -4.170800 \\
\hline $\mathrm{H}$ & 3.116400 & -1.554500 & -4.890300 \\
\hline C & 1.396500 & -0.179600 & -2.331500 \\
\hline C & 2.586900 & 0.741600 & -2.606600 \\
\hline $\mathrm{H}$ & 3.246600 & 0.613800 & -3.483800 \\
\hline C & 2.718200 & 1.895300 & -1.865200 \\
\hline $\mathrm{H}$ & 3.517100 & 2.614500 & -2.132000 \\
\hline C & 2.112200 & 3.511200 & -0.164900 \\
\hline C & 3.480900 & 3.550700 & 0.584000 \\
\hline $\mathrm{H}$ & 4.305200 & 3.335300 & -0.133700 \\
\hline $\mathrm{H}$ & 3.489300 & 2.741300 & 1.349000 \\
\hline C & 2.096600 & 4.677200 & -1.206000 \\
\hline $\mathrm{H}$ & 1.115800 & 4.667000 & -1.729500 \\
\hline $\mathrm{H}$ & 2.872300 & 4.510300 & -1.983800 \\
\hline C & 1.004000 & 3.819100 & 0.868800 \\
\hline $\mathrm{H}$ & 0.017400 & 3.797700 & 0.360600 \\
\hline $\mathrm{H}$ & 0.984100 & 3.018800 & 1.639000 \\
\hline C & 3.719200 & 4.921000 & 1.259500 \\
\hline $\mathrm{H}$ & 4.703900 & 4.912200 & 1.778800 \\
\hline C & 3.708700 & 6.032100 & 0.184400 \\
\hline $\mathrm{H}$ & 3.905500 & 7.020900 & 0.655300 \\
\hline $\mathrm{H}$ & 4.525100 & 5.861100 & -0.553200 \\
\hline C & 2.339600 & 6.045500 & -0.532600 \\
\hline $\mathrm{H}$ & 2.334800 & 6.841600 & -1.310300 \\
\hline C & 1.220000 & 6.312200 & 0.494300 \\
\hline $\mathrm{H}$ & 1.366500 & 7.302200 & 0.981600 \\
\hline $\mathrm{H}$ & 0.233000 & 6.347800 & -0.018000 \\
\hline C & 1.231000 & 5.187700 & 1.552800 \\
\hline $\mathrm{H}$ & 0.417700 & 5.358000 & 2.289600 \\
\hline C & 2.592300 & 5.183500 & 2.282600 \\
\hline H & 2.757300 & 6.157700 & 2.794600 \\
\hline $\mathrm{H}$ & 2.601300 & 4.400000 & 3.072400 \\
\hline $\mathrm{N}$ & -3.134000 & -1.411400 & 0.628700 \\
\hline $\mathrm{N}$ & 1.876800 & 2.200200 & -0.813500 \\
\hline 0 & -1.026900 & 0.628400 & -0.245800 \\
\hline Si & -3.045600 & 2.736100 & 2.330100 \\
\hline Si & -2.999500 & 0.181700 & 4.214900 \\
\hline Si & 0.106100 & 0.430300 & -3.619600 \\
\hline $\mathrm{Si}$ & 1.890200 & -1.991900 & -2.694200 \\
\hline $\mathrm{K}$ & -3.451700 & 0.878400 & -1.352800 \\
\hline K & 4.316800 & -0.441300 & -0.449600 \\
\hline C & 0.069300 & -0.857200 & 2.118900 \\
\hline C & 1.199600 & 0.077000 & 1.542600 \\
\hline C & 0.478900 & -2.293200 & 2.285100 \\
\hline C & 2.585800 & -0.255000 & 1.968000 \\
\hline $\mathrm{H}$ & 3.338900 & 0.557600 & 2.041800 \\
\hline C & 1.802800 & -2.607100 & 2.273800 \\
\hline
\end{tabular}




\begin{tabular}{|c|c|c|c|}
\hline $\mathrm{C}$ & 2.875500 & -1.558900 & 2.242900 \\
\hline $\mathrm{H}$ & 0.978600 & 1.121300 & 1.865600 \\
\hline $\mathrm{H}$ & -0.170800 & -0.528100 & 3.165800 \\
\hline $\mathrm{H}$ & -0.283800 & -3.048200 & 2.550700 \\
\hline $\mathrm{C}$ & 2.842300 & -3.617300 & 2.600200 \\
\hline $\mathrm{C}$ & 3.866000 & -2.596200 & 2.615800 \\
\hline$C$ & 5.191900 & -2.892100 & 2.956900 \\
\hline $\mathrm{H}$ & 5.981900 & -2.125200 & 3.019300 \\
\hline $\mathrm{C}$ & 3.134100 & -4.951500 & 2.895700 \\
\hline $\mathrm{C}$ & 4.482500 & -5.248000 & 3.216200 \\
\hline$C$ & 5.481000 & -4.248400 & 3.250300 \\
\hline $\mathrm{H}$ & 6.510200 & -4.533800 & 3.523500 \\
\hline $\mathrm{H}$ & 2.369500 & -5.744600 & 2.890600 \\
\hline $\mathrm{H}$ & 4.762700 & -6.286400 & 3.457300 \\
\hline $\mathrm{C}$ & 7.406400 & 0.596400 & -1.810200 \\
\hline C & 7.257200 & -0.703100 & -2.333600 \\
\hline $\mathrm{C}$ & 7.307500 & -1.819300 & -1.474900 \\
\hline$C$ & 7.508200 & -1.637100 & -0.092000 \\
\hline$C$ & 7.661500 & -0.337000 & 0.430400 \\
\hline $\mathrm{C}$ & 7.610500 & 0.779400 & -0.428100 \\
\hline $\mathrm{H}$ & 7.372000 & 1.468100 & -2.482600 \\
\hline $\mathrm{H}$ & 7.107100 & -0.847200 & -3.415200 \\
\hline $\mathrm{H}$ & 7.196000 & -2.835400 & -1.885300 \\
\hline $\mathrm{H}$ & 7.544400 & -2.509200 & 0.579700 \\
\hline $\mathrm{H}$ & 7.830900 & -0.194000 & 1.509700 \\
\hline $\mathrm{H}$ & 7.739300 & 1.794500 & -0.019700 \\
\hline $\mathrm{H}$ & -5.163500 & 4.267500 & -2.864200 \\
\hline $\mathrm{C}$ & -5.498600 & 3.225500 & -2.987900 \\
\hline $\mathrm{C}$ & -6.369200 & 2.648900 & -2.042000 \\
\hline$C$ & -5.061600 & 2.471200 & -4.094900 \\
\hline $\mathrm{C}$ & -6.802300 & 1.317800 & -2.202400 \\
\hline $\mathrm{H}$ & -6.714000 & 3.238900 & -1.178400 \\
\hline $\mathrm{C}$ & -5.495400 & 1.140300 & -4.255800 \\
\hline $\mathrm{H}$ & -4.386100 & 2.924200 & -4.837800 \\
\hline C & -6.365900 & 0.563700 & -3.309600 \\
\hline $\mathrm{H}$ & -7.484500 & 0.868800 & -1.463400 \\
\hline $\mathrm{H}$ & -5.160100 & 0.552700 & -5.125100 \\
\hline $\mathrm{H}$ & -6.709500 & -0.474900 & -3.438500 \\
\hline
\end{tabular}

Table S16. Cartesian coordinates of TS-3

Zero-point correction=

Thermal correction to Energy=

1.328869 (Hartree/Particle)

Thermal correction to Enthalpy=

1.413889

Thermal correction to Gibbs Free Energy $=11.204454$

Sum of electronic and zero-point Energies $=-5441.534410$

Sum of electronic and thermal Energies $=\quad-5441.449390$

Sum of electronic and thermal Enthalpies $=\quad-5441.448446$

Sum of electronic and thermal Free Energies $=-5441.658825$

$\begin{array}{lrrr}\text { Al } & 1.830600 & 0.073600 & -1.302100 \\ \text { Al } & -0.931600 & 0.171400 & 0.366900 \\ \text { C } & 1.304000 & 4.370400 & -2.561100 \\ \text { H } & 0.428800 & 3.733600 & -2.798000 \\ \text { H } & 0.939000 & 5.289300 & -2.055500 \\ \text { H } & 1.760000 & 4.682300 & -3.523100 \\ \text { C } & 1.942700 & 3.611200 & 0.345400 \\ \text { H } & 2.773700 & 3.522200 & 1.080800 \\ \text { H } & 1.488500 & 4.608900 & 0.525400 \\ \text { H } & 1.176800 & 2.835100 & 0.551300 \\ \text { C } & 4.115900 & 4.637600 & -1.447800 \\ \text { H } & 4.590800 & 4.731700 & -2.444800 \\ \text { H } & 3.810400 & 5.655800 & -1.121800\end{array}$




\begin{tabular}{|c|c|c|c|}
\hline-1 & 4.893700 & 4.281400 & -0.739800 \\
\hline C & 1.717000 & 1.962500 & -4.967300 \\
\hline $\mathrm{H}$ & 1.524300 & 3.051000 & -5.047600 \\
\hline $\mathrm{H}$ & 1.905400 & 1.584000 & -5.995400 \\
\hline $\mathrm{H}$ & 0.785700 & 1.480900 & -4.606500 \\
\hline C & 3.836300 & -0.169200 & -4.374700 \\
\hline $\mathrm{H}$ & 3.053600 & -0.942000 & -4.236100 \\
\hline $\mathrm{H}$ & 4.123900 & -0.173000 & -5.448000 \\
\hline $\mathrm{H}$ & 4.723900 & -0.468000 & -3.782000 \\
\hline C & 4.615600 & 2.749900 & -4.522300 \\
\hline $\mathrm{H}$ & 5.564800 & 2.631200 & -3.959800 \\
\hline $\mathrm{H}$ & 4.830000 & 2.521000 & -5.588700 \\
\hline $\mathrm{H}$ & 4.317600 & 3.817700 & -4.470100 \\
\hline C & 2.980500 & 1.707400 & -1.981000 \\
\hline C & 4.334400 & 1.327000 & -1.388200 \\
\hline $\mathrm{H}$ & 5.210300 & 1.998200 & -1.448900 \\
\hline C & 4.530300 & 0.065100 & -0.884000 \\
\hline $\mathrm{H}$ & 5.544600 & -0.247800 & -0.567600 \\
\hline C & 3.853100 & -2.253800 & -0.526300 \\
\hline C & 2.580300 & -3.102400 & -0.334800 \\
\hline $\mathrm{H}$ & 1.912900 & -2.979700 & -1.213600 \\
\hline $\mathrm{H}$ & 2.015200 & -2.709400 & 0.536300 \\
\hline C & 4.638900 & -2.832900 & -1.741700 \\
\hline $\mathrm{H}$ & 5.564300 & -2.237500 & -1.905200 \\
\hline $\mathrm{H}$ & 4.017200 & -2.708500 & -2.653600 \\
\hline C & 4.735900 & -2.450000 & 0.748000 \\
\hline $\mathrm{H}$ & 4.180300 & -2.069600 & 1.637400 \\
\hline $\mathrm{H}$ & 5.673000 & -1.856100 & 0.666600 \\
\hline C & 2.917100 & -4.595200 & -0.123100 \\
\hline $\mathrm{H}$ & 1.970000 & -5.162800 & 0.002200 \\
\hline C & 3.688600 & -5.131600 & -1.349900 \\
\hline $\mathrm{H}$ & 3.059900 & -5.046900 & -2.263400 \\
\hline $\mathrm{H}$ & 3.919800 & -6.212100 & -1.216200 \\
\hline C & 4.992000 & -4.321400 & -1.527700 \\
\hline $\mathrm{H}$ & 5.549800 & -4.698300 & -2.413800 \\
\hline C & 5.868600 & -4.466700 & -0.264100 \\
\hline $\mathrm{H}$ & 6.149000 & -5.532400 & -0.108300 \\
\hline $\mathrm{H}$ & 6.819100 & -3.900700 & -0.389300 \\
\hline C & 5.092200 & -3.940000 & 0.963800 \\
\hline $\mathrm{H}$ & 5.723900 & -4.037600 & 1.875400 \\
\hline C & 3.792100 & -4.754500 & 1.140200 \\
\hline $\mathrm{H}$ & 4.031800 & -5.827600 & 1.312500 \\
\hline $\mathrm{H}$ & 3.238000 & -4.401100 & 2.038500 \\
\hline C & 0.205100 & 1.473600 & 3.308300 \\
\hline $\mathrm{H}$ & -0.704800 & 2.054700 & 3.562700 \\
\hline $\mathrm{H}$ & 0.979400 & 1.737100 & 4.063200 \\
\hline $\mathrm{H}$ & 0.532600 & 1.817400 & 2.308200 \\
\hline C & 1.404900 & -1.415600 & 3.345300 \\
\hline $\mathrm{H}$ & 1.747500 & -1.585700 & 2.304000 \\
\hline $\mathrm{H}$ & 2.219000 & -0.941600 & 3.938600 \\
\hline $\mathrm{H}$ & 1.241600 & -2.418300 & 3.792100 \\
\hline C & -0.839400 & -0.530600 & 5.211500 \\
\hline $\mathrm{H}$ & -1.056400 & -1.565400 & 5.536900 \\
\hline $\mathrm{H}$ & -0.058700 & -0.121000 & 5.889300 \\
\hline $\mathrm{H}$ & -1.755500 & 0.077900 & 5.359300 \\
\hline C & -3.527100 & -3.332700 & 1.523900 \\
\hline $\mathrm{H}$ & -3.631500 & -3.305000 & 0.419000 \\
\hline $\mathrm{H}$ & -3.680300 & -4.394000 & 1.813300 \\
\hline $\mathrm{H}$ & -4.342700 & -2.760300 & 2.019200 \\
\hline C & -0.535400 & -3.821100 & 1.128100 \\
\hline $\mathrm{H}$ & 0.469400 & -3.787500 & 1.593800 \\
\hline $\mathrm{H}$ & -0.855300 & -4.883900 & 1.091700 \\
\hline $\mathrm{H}$ & -0.452800 & -3.452100 & 0.084900 \\
\hline C & -1.758800 & -3.547400 & 3.896800 \\
\hline $\mathrm{H}$ & -2.513100 & -3.082400 & 4.565800 \\
\hline $\mathrm{H}$ & -2.005000 & -4.629000 & 3.824200 \\
\hline $\mathrm{H}$ & -0.770800 & -3.474300 & 4.395300 \\
\hline & -1.489200 & -0.919600 & 2.071700 \\
\hline
\end{tabular}




\begin{tabular}{|c|c|c|c|}
\hline C & -2.717300 & -0.148600 & 2.522600 \\
\hline $\mathrm{H}$ & -3.326400 & -0.456500 & 3.392300 \\
\hline C & -3.004000 & 1.047500 & 1.914000 \\
\hline $\mathrm{H}$ & -3.834400 & 1.670100 & 2.295800 \\
\hline C & -2.669900 & 2.865200 & 0.336900 \\
\hline C & -4.179200 & 2.939800 & -0.062000 \\
\hline $\mathrm{H}$ & -4.822600 & 2.647900 & 0.797000 \\
\hline $\mathrm{H}$ & -4.368600 & 2.207700 & -0.882400 \\
\hline C & -2.407000 & 3.934400 & 1.443500 \\
\hline $\mathrm{H}$ & -1.329200 & 3.899600 & 1.713300 \\
\hline $\mathrm{H}$ & -2.973000 & 3.671300 & 2.363700 \\
\hline C & -1.854900 & 3.277100 & -0.910400 \\
\hline H & -0.771800 & 3.222000 & -0.672800 \\
\hline $\mathrm{H}$ & -2.045500 & 2.556600 & -1.732300 \\
\hline C & -4.573200 & 4.358700 & -0.534600 \\
\hline $\mathrm{H}$ & -5.654600 & 4.370700 & -0.798300 \\
\hline C & -4.305200 & 5.370100 & 0.603500 \\
\hline $\mathrm{H}$ & -4.609300 & 6.391300 & 0.283500 \\
\hline H & -4.921900 & 5.117800 & 1.495400 \\
\hline C & -2.804100 & 5.350400 & 0.971000 \\
\hline $\mathrm{H}$ & -2.612100 & 6.077500 & 1.791100 \\
\hline C & -1.964500 & 5.725700 & -0.268400 \\
\hline H & -2.221600 & 6.751100 & -0.615800 \\
\hline $\mathrm{H}$ & -0.883300 & 5.734900 & -0.008300 \\
\hline C & -2.232900 & 4.699500 & -1.389900 \\
\hline $\mathrm{H}$ & -1.617500 & 4.949700 & -2.279800 \\
\hline C & -3.729000 & 4.734800 & -1.770200 \\
\hline $\mathrm{H}$ & -4.010400 & 5.747400 & -2.135400 \\
\hline $\mathrm{H}$ & -3.930800 & 4.026300 & -2.604400 \\
\hline $\mathrm{N}$ & 3.479500 & -0.839300 & -0.742800 \\
\hline $\mathrm{N}$ & -2.286300 & 1.510300 & 0.810100 \\
\hline 0 & 0.808000 & 0.370600 & 0.174800 \\
\hline Si & 2.584500 & 3.489000 & -1.458900 \\
\hline Si & 3.246400 & 1.574800 & -3.886400 \\
\hline Si & -0.212000 & -0.391000 & 3.411600 \\
\hline $\mathrm{Si}$ & -1.785500 & -2.818300 & 2.124300 \\
\hline K & 3.075800 & 0.832800 & 1.676400 \\
\hline K & -4.515000 & -0.779500 & 0.038700 \\
\hline C & 0.444100 & -0.671400 & -2.621200 \\
\hline C & -0.734800 & 0.234300 & -2.323900 \\
\hline C & 0.073200 & -2.133700 & -2.685900 \\
\hline C & -2.003500 & -0.224800 & -1.944400 \\
\hline H & -2.822300 & 0.520300 & -1.916100 \\
\hline C & -1.132800 & -2.553300 & -2.222500 \\
\hline C & -2.228000 & -1.625800 & -1.749300 \\
\hline $\mathrm{H}$ & -0.608100 & 1.308600 & -2.533300 \\
\hline $\mathrm{H}$ & 0.878000 & -0.375200 & -3.605100 \\
\hline $\mathrm{H}$ & 0.754400 & -2.822900 & -3.215200 \\
\hline C & -2.140900 & -3.631300 & -2.366600 \\
\hline C & -3.199200 & -2.681300 & -2.044400 \\
\hline C & -4.553000 & -3.072500 & -2.181800 \\
\hline $\mathrm{H}$ & -5.412300 & -2.382200 & -2.089500 \\
\hline C & -2.398200 & -4.949500 & -2.720400 \\
\hline C & -3.764500 & -5.347900 & -2.782300 \\
\hline C & -4.801500 & -4.423100 & -2.538200 \\
\hline $\mathrm{H}$ & -5.846900 & -4.758400 & -2.649200 \\
\hline $\mathrm{H}$ & -1.594500 & -5.663500 & -2.964900 \\
\hline $\mathrm{H}$ & -4.018900 & -6.384800 & -3.053400 \\
\hline C & -7.685000 & 0.747500 & 0.668300 \\
\hline C & -7.595100 & -0.246000 & 1.663900 \\
\hline C & -7.635200 & -1.608200 & 1.305500 \\
\hline C & -7.765700 & -1.978200 & -0.047700 \\
\hline C & -7.854800 & -0.984000 & -1.043400 \\
\hline C & -7.814100 & 0.377900 & -0.685500 \\
\hline $\mathrm{H}$ & -7.664800 & 1.812400 & 0.948900 \\
\hline $\mathrm{H}$ & -7.503200 & 0.042400 & 2.723200 \\
\hline $\mathrm{H}$ & -7.572000 & -2.385200 & 2.083800 \\
\hline $\mathrm{H}$ & -7.797200 & -3.042800 & -0.327300 \\
\hline
\end{tabular}




$\begin{array}{rrrr}\text { H } & -7.965700 & -1.272700 & -2.100700 \\ \text { H } & -7.894200 & 1.154100 & -1.463300 \\ \text { H } & 3.823100 & 3.930800 & 4.297000 \\ \mathrm{C} & 4.430300 & 3.031300 & 4.108500 \\ \mathrm{C} & 5.390200 & 3.033200 & 3.077400 \\ \mathrm{C} & 4.252500 & 1.881700 & 4.903800 \\ \mathrm{C} & 6.172000 & 1.885200 & 2.839900 \\ \mathrm{H} & 5.533200 & 3.933000 & 2.458600 \\ \mathrm{C} & 5.035000 & 0.734200 & 4.667400 \\ \mathrm{H} & 3.508900 & 1.883600 & 5.716400 \\ \mathrm{C} & 5.994400 & 0.735800 & 3.635400 \\ \mathrm{H} & 6.923000 & 1.888100 & 2.034700 \\ \mathrm{H} & 4.903500 & -0.161600 & 5.295000 \\ \mathrm{H} & 6.610200 & -0.159300 & 3.454800\end{array}$

\section{Table S17. Cartesian coordinates of Int-5}

Zero-point correction=

Thermal correction to Energy=

Thermal correction to Enthalpy=

Thermal correction to Gibbs Free Energy=

Sum of electronic and zero-point Energies=

Sum of electronic and thermal Energies=

Sum of electronic and thermal Enthalpies $=\quad-5441.482667$

Sum of electronic and thermal Free Energies $=-5441.693591$

\subsection{3 (Hartree/Particle)}

1.415474

1.416418

1.205495

$-5441.568732$

$-5441.483612$

$\begin{array}{lrrr}\text { Al } & 2.044200 & 0.410800 & -1.244100 \\ \text { Al } & -1.019300 & -0.090600 & 0.010500 \\ \text { C } & 1.434500 & 4.711000 & -1.524000 \\ \text { H } & 0.628000 & 4.069400 & -1.932800 \\ \text { H } & 0.968700 & 5.523100 & -0.926600 \\ \text { H } & 1.957900 & 5.188100 & -2.378200 \\ \text { C } & 1.799500 & 3.355100 & 1.226900 \\ \text { H } & 2.552100 & 3.122800 & 2.013500 \\ \text { H } & 1.249400 & 4.250900 & 1.585800 \\ \text { H } & 1.076800 & 2.519000 & 1.122300 \\ \text { C } & 4.032600 & 4.951500 & 0.050900 \\ \text { H } & 4.527500 & 5.435000 & -0.814300 \\ \text { H } & 3.590000 & 5.757500 & 0.676600 \\ \text { H } & 4.823500 & 4.460500 & 0.656700 \\ \text { C } & 2.899000 & 3.055300 & -4.410000 \\ \text { H } & 2.220100 & 3.877500 & -4.109800 \\ \text { H } & 3.534800 & 3.434200 & -5.239800 \\ \text { H } & 2.282100 & 2.232400 & -4.823000 \\ \text { C } & 4.923800 & 0.914800 & -3.706300 \\ \text { H } & 4.221900 & 0.076300 & -3.886800 \\ \text { H } & 5.397900 & 1.173800 & -4.677300 \\ \text { H } & 5.717100 & 0.554100 & -3.021900 \\ \text { C } & 5.432400 & 3.788700 & -2.927600 \\ \text { H } & 6.131700 & 3.625600 & -2.082200 \\ \text { H } & 6.027100 & 3.755200 & -3.866200 \\ \text { H } & 5.018000 & 4.814100 & -2.837000 \\ \text { C } & 3.275400 & 2.127900 & -1.271600 \\ \text { C } & 4.470300 & 1.601300 & -0.481100 \\ \text { H } & 5.298000 & 2.260400 & -0.159900 \\ \text { C } & 4.614600 & 0.249200 & -0.287800 \\ \text { H } & 5.548800 & -0.139800 & 0.163300 \\ \text { C } & 4.002200 & -2.073500 & -0.725600 \\ \text { C } & 2.753300 & -2.945500 & -0.970800 \\ \text { H } & 2.237300 & -2.592500 & -1.887400 \\ \text { H } & 2.033500 & -2.794100 & -0.140000 \\ \text { C } & 4.983200 & -2.310900 & -1.913800 \\ \text { H } & 5.896500 & -1.692200 & -1.770800\end{array}$




\begin{tabular}{|c|c|c|c|}
\hline $\mathrm{H}$ & 4.499700 & -1.949100 & -2.845900 \\
\hline C & 4.694300 & -2.605300 & 0.570900 \\
\hline $\mathrm{H}$ & 4.004000 & -2.471500 & 1.435500 \\
\hline $\mathrm{H}$ & 5.611000 & -2.016600 & 0.792200 \\
\hline C & 3.116100 & -4.441300 & -1.105100 \\
\hline $\mathrm{H}$ & 2.185000 & -5.022800 & -1.280600 \\
\hline C & 4.083700 & -4.634400 & -2.293800 \\
\hline $\mathrm{H}$ & 3.596100 & -4.314600 & -3.241000 \\
\hline $\mathrm{H}$ & 4.339600 & -5.711600 & -2.411100 \\
\hline C & 5.362700 & -3.802800 & -2.050000 \\
\hline $\mathrm{H}$ & 6.061300 & -3.928000 & -2.907200 \\
\hline C & 6.046800 & -4.280100 & -0.749600 \\
\hline $\mathrm{H}$ & 6.344100 & -5.349000 & -0.838200 \\
\hline $\mathrm{H}$ & 6.980900 & -3.701000 & -0.572600 \\
\hline C & 5.074600 & -4.098500 & 0.437900 \\
\hline $\mathrm{H}$ & 5.567100 & -4.435500 & 1.377900 \\
\hline C & 3.798000 & -4.930600 & 0.192400 \\
\hline $\mathrm{H}$ & 4.052200 & -6.011300 & 0.112000 \\
\hline $\mathrm{H}$ & 3.102000 & -4.827400 & 1.054700 \\
\hline C & -0.124500 & 0.401100 & 3.433300 \\
\hline $\mathrm{H}$ & -1.055200 & 0.814400 & 3.872300 \\
\hline $\mathrm{H}$ & 0.658300 & 0.480900 & 4.221200 \\
\hline $\mathrm{H}$ & 0.125300 & 1.058200 & 2.577400 \\
\hline C & 1.198900 & -2.369700 & 2.762900 \\
\hline $\mathrm{H}$ & 1.649600 & -2.220700 & 1.760300 \\
\hline $\mathrm{H}$ & 1.939500 & -2.106400 & 3.551500 \\
\hline $\mathrm{H}$ & 1.019800 & -3.460900 & 2.862500 \\
\hline C & -1.172700 & -2.100300 & 4.614500 \\
\hline $\mathrm{H}$ & -1.335600 & -3.195100 & 4.617800 \\
\hline $\mathrm{H}$ & -0.451800 & -1.867500 & 5.429100 \\
\hline $\mathrm{H}$ & -2.131700 & -1.608400 & 4.877800 \\
\hline C & -3.545400 & -3.660000 & 0.002500 \\
\hline $\mathrm{H}$ & -3.606200 & -3.107900 & -0.957700 \\
\hline $\mathrm{H}$ & -3.604300 & -4.736900 & -0.263800 \\
\hline $\mathrm{H}$ & -4.433900 & -3.458100 & 0.641700 \\
\hline C & -0.521000 & -4.132300 & -0.099400 \\
\hline $\mathrm{H}$ & 0.411600 & -4.236900 & 0.490100 \\
\hline $\mathrm{H}$ & -0.810000 & -5.144900 & -0.452800 \\
\hline $\mathrm{H}$ & -0.299100 & -3.500500 & -0.983200 \\
\hline C & -2.134700 & -4.590600 & 2.432400 \\
\hline $\mathrm{H}$ & -2.919400 & -4.253400 & 3.142000 \\
\hline $\mathrm{H}$ & -2.446300 & -5.582600 & 2.038500 \\
\hline $\mathrm{H}$ & -1.202400 & -4.750000 & 3.012900 \\
\hline C & -1.609500 & -1.570000 & 1.448700 \\
\hline C & -2.861500 & -0.925400 & 2.038600 \\
\hline $\mathrm{H}$ & -3.471800 & -1.417200 & 2.821200 \\
\hline C & -3.096500 & 0.407500 & 1.778900 \\
\hline $\mathrm{H}$ & -3.923400 & 0.918900 & 2.312900 \\
\hline C & -2.777600 & 2.533100 & 0.654100 \\
\hline C & -4.136700 & 2.574900 & -0.115400 \\
\hline $\mathrm{H}$ & -4.916000 & 2.051100 & 0.488600 \\
\hline $\mathrm{H}$ & -4.014700 & 2.022700 & -1.075600 \\
\hline C & -2.954200 & 3.336500 & 1.980900 \\
\hline $\mathrm{H}$ & -1.986700 & 3.325300 & 2.529000 \\
\hline $\mathrm{H}$ & -3.700200 & 2.845700 & 2.642800 \\
\hline C & -1.732400 & 3.292200 & -0.193300 \\
\hline $\mathrm{H}$ & -0.770000 & 3.298800 & 0.356500 \\
\hline $\mathrm{H}$ & -1.548100 & 2.739200 & -1.136800 \\
\hline C & -4.597000 & 4.023700 & -0.392400 \\
\hline $\mathrm{H}$ & -5.569400 & 4.009400 & -0.935100 \\
\hline C & -4.761200 & 4.770600 & 0.950500 \\
\hline $\mathrm{H}$ & -5.112200 & 5.810600 & 0.767800 \\
\hline $\mathrm{H}$ & -5.540900 & 4.275100 & 1.572800 \\
\hline C & -3.410400 & 4.788600 & 1.703300 \\
\hline $\mathrm{H}$ & -3.533000 & 5.323100 & 2.671700 \\
\hline C & -2.354800 & 5.508700 & 0.837800 \\
\hline $\mathrm{H}$ & -2.667200 & 6.559200 & 0.643300 \\
\hline $\mathrm{H}$ & -1.384200 & 5.556500 & 1.378900 \\
\hline
\end{tabular}




\begin{tabular}{|c|c|c|c|}
\hline C & -2.184900 & 4.740700 & -0.490400 \\
\hline $\mathrm{H}$ & -1.411200 & 5.236900 & -1.112800 \\
\hline C & -3.527800 & 4.730500 & -1.253200 \\
\hline $\mathrm{H}$ & -3.848500 & 5.770600 & -1.485100 \\
\hline $\mathrm{H}$ & -3.412700 & 4.205800 & -2.227600 \\
\hline $\mathrm{N}$ & 3.605200 & -0.651400 & -0.618100 \\
\hline $\mathrm{N}$ & -2.336800 & 1.137800 & 0.884900 \\
\hline 0 & 0.727000 & 0.308200 & -0.007800 \\
\hline $\mathrm{Si}$ & 2.653100 & 3.720500 & -0.449700 \\
\hline $\mathrm{Si}$ & 4.056200 & 2.459400 & -3.008700 \\
\hline $\mathrm{Si}$ & -0.458400 & -1.429200 & 2.969800 \\
\hline $\mathrm{Si}$ & -1.904300 & -3.386500 & 0.957200 \\
\hline K & 2.692200 & 0.348000 & 2.009600 \\
\hline $\mathrm{K}$ & -4.960700 & -0.865500 & -0.157400 \\
\hline C & 1.190900 & -0.041600 & -3.063100 \\
\hline C & 0.196800 & 1.089200 & -3.206000 \\
\hline C & 0.493700 & -1.361400 & -3.156200 \\
\hline C & -1.085000 & 0.991300 & -2.761200 \\
\hline $\mathrm{H}$ & -1.806300 & 1.799400 & -2.972700 \\
\hline C & -0.766400 & -1.466400 & -2.654800 \\
\hline C & -1.539800 & -0.257100 & -2.058600 \\
\hline $\mathrm{H}$ & 0.526800 & 2.006400 & -3.718100 \\
\hline $\mathrm{H}$ & 1.999800 & 0.035900 & -3.818100 \\
\hline $\mathrm{H}$ & 0.938100 & -2.170100 & -3.762700 \\
\hline C & -2.033700 & -2.143400 & -2.979300 \\
\hline C & -2.774000 & -0.976000 & -2.593200 \\
\hline C & -4.113000 & -0.825700 & -2.973100 \\
\hline $\mathrm{H}$ & -4.661000 & 0.130900 & -2.868000 \\
\hline C & -2.645200 & -3.243500 & -3.593500 \\
\hline C & -4.030200 & -3.127000 & -3.873400 \\
\hline C & -4.743100 & -1.940700 & -3.598800 \\
\hline $\mathrm{H}$ & -5.795200 & -1.860800 & -3.920100 \\
\hline $\mathrm{H}$ & -2.087000 & -4.144900 & -3.893400 \\
\hline $\mathrm{H}$ & -4.554000 & -3.963300 & -4.364600 \\
\hline C & -7.882700 & -0.255900 & 1.618200 \\
\hline C & -7.733800 & -1.650900 & 1.750400 \\
\hline C & -7.911400 & -2.487700 & 0.630600 \\
\hline C & -8.237000 & -1.929500 & -0.621700 \\
\hline C & -8.385800 & -0.534400 & -0.753900 \\
\hline C & -8.209000 & 0.302500 & 0.366100 \\
\hline $\mathrm{H}$ & -7.752800 & 0.397200 & 2.495600 \\
\hline $\mathrm{H}$ & -7.485800 & -2.087900 & 2.730700 \\
\hline $\mathrm{H}$ & -7.802700 & -3.578800 & 0.735000 \\
\hline $\mathrm{H}$ & -8.381500 & -2.584500 & -1.495300 \\
\hline $\mathrm{H}$ & -8.650700 & -0.099200 & -1.730700 \\
\hline $\mathrm{H}$ & -8.336400 & 1.392000 & 0.265500 \\
\hline $\mathrm{H}$ & 2.988500 & 2.375700 & 5.722600 \\
\hline C & 3.685200 & 1.660700 & 5.256900 \\
\hline C & 4.649000 & 2.115100 & 4.335600 \\
\hline C & 3.616000 & 0.293000 & 5.587400 \\
\hline C & 5.543900 & 1.201900 & 3.743600 \\
\hline $\mathrm{H}$ & 4.707600 & 3.185000 & 4.080900 \\
\hline C & 4.512000 & -0.620100 & 4.997300 \\
\hline $\mathrm{H}$ & 2.866800 & -0.061300 & 6.313000 \\
\hline C & 5.475600 & -0.165700 & 4.075700 \\
\hline $\mathrm{H}$ & 6.297400 & 1.557600 & 3.023700 \\
\hline $\mathrm{H}$ & 4.464500 & -1.688300 & 5.262300 \\
\hline $\mathrm{H}$ & 6.178500 & -0.879000 & 3.617200 \\
\hline
\end{tabular}

Table S18. Cartesian coordinates of TS-4

Zero-point correction=

Thermal correction to Energy=

1.329333 (Hartree/Particle)

Thermal correction to Enthalpy= 
Thermal correction to Gibbs Free Energy=

1.207044

Sum of electronic and zero-point Energies $=-5441.530266$

Sum of electronic and thermal Energies= $\quad-5441.445582$

Sum of electronic and thermal Enthalpies $=-5441.444638$

Sum of electronic and thermal Free Energies $=-5441.652555$

\begin{tabular}{|c|c|c|c|}
\hline Al & 1.498100 & 0.436800 & -1.298500 \\
\hline $\mathrm{Al}$ & -1.135800 & -0.149100 & 0.503700 \\
\hline C & 0.879100 & 4.670900 & -2.02910 \\
\hline $\mathrm{H}$ & 0.005400 & 4.009100 & -2.1900 \\
\hline $\mathrm{H}$ & 0.547400 & 5.560200 & -1.45240 \\
\hline $\mathrm{H}$ & 1.223600 & 5.030300 & $-3.0204 e$ \\
\hline$C$ & 1.783800 & 3.654300 & 0.7424 \\
\hline $\mathrm{H}$ & 2.680700 & 3.491900 & 1.3811 \\
\hline $\mathrm{H}$ & 1.335100 & 4.612500 & 1.0807 \\
\hline $\mathrm{H}$ & 1.040700 & 2.848000 & 0.9117 \\
\hline C & 3.741500 & 5.006200 & -1.06790 \\
\hline $\mathrm{H}$ & 4.047000 & 5.349300 & -2.07616 \\
\hline $\mathrm{H}$ & 3.439000 & 5.905400 & $-0.4875 e$ \\
\hline $\mathrm{H}$ & 4.638700 & 4.579200 & -0.5725 \\
\hline$C$ & 1.939100 & 2.757400 & $-4.9773 e$ \\
\hline $\mathrm{H}$ & 1.483900 & 3.733100 & -4.7143 \\
\hline $\mathrm{H}$ & 2.481100 & 2.895400 & -5.9385 \\
\hline $\mathrm{H}$ & 1.117600 & 2.038000 & $-5.1656 e$ \\
\hline$C$ & 3.795300 & 0.448600 & -4.34546 \\
\hline $\mathrm{H}$ & 2.977400 & -0.295600 & -4.43430 \\
\hline $\mathrm{H}$ & 4.222800 & 0.587400 & $-5.3619 \ell$ \\
\hline $\mathrm{H}$ & 4.584100 & 0.014100 & -3.70090 \\
\hline$C$ & 4.713100 & 3.306200 & -3.97440 \\
\hline $\mathrm{H}$ & 5.527700 & 3.158100 & -3.23700 \\
\hline $\mathrm{H}$ & 5.135500 & 3.103900 & -4.98250 \\
\hline $\mathrm{H}$ & 4.420200 & 4.376200 & -3.95216 \\
\hline$C$ & 2.717900 & 2.067700 & -1.81990 \\
\hline$C$ & 4.032000 & 1.605100 & -1.20280 \\
\hline $\mathrm{H}$ & 4.915800 & 2.264200 & -1.13140 \\
\hline C & 4.193100 & 0.280000 & -0.88660 \\
\hline $\mathrm{H}$ & 5.190300 & -0.084900 & -0.57640 \\
\hline$C$ & 3.521800 & -2.056300 & -0.84090 \\
\hline$C$ & 2.263100 & -2.934100 & -0.70700 \\
\hline $\mathrm{H}$ & 1.592800 & -2.741200 & -1.56700 \\
\hline $\mathrm{H}$ & 1.694900 & -2.627500 & 0.19750 \\
\hline$C$ & 4.293400 & -2.513900 & -2.11570 \\
\hline $\mathrm{H}$ & 5.209500 & -1.893900 & -2.23480 \\
\hline $\mathrm{H}$ & 3.657000 & -2.313000 & $-3.0036 e$ \\
\hline$C$ & 4.431600 & -2.363000 & 0.3940 \\
\hline $\mathrm{H}$ & 3.895100 & -2.070200 & 1.32630 \\
\hline $\mathrm{H}$ & 5.367300 & -1.764800 & 0.35450 \\
\hline$C$ & 2.619700 & -4.436900 & -0.64030 \\
\hline $\mathrm{H}$ & 1.680600 & -5.023600 & -0.55740 \\
\hline$C$ & 3.375400 & -4.850200 & -1.92170 \\
\hline $\mathrm{H}$ & 2.728400 & -4.693000 & -2.81180 \\
\hline $\mathrm{H}$ & 3.621000 & -5.935500 & -1.88980 \\
\hline$C$ & 4.666500 & -4.011700 & $-2.0485 e$ \\
\hline $\mathrm{H}$ & 5.212400 & -4.296800 & -2.9754 \\
\hline$C$ & 5.566300 & -4.262900 & -0.8183 \\
\hline $\mathrm{H}$ & 5.861300 & -5.334600 & -0.76700 \\
\hline $\mathrm{H}$ & 6.507600 & -3.674500 & $-0.9037 e$ \\
\hline$C$ & 4.804000 & -3.862800 & 0.46470 \\
\hline $\mathrm{H}$ & 5.452900 & -4.034300 & 1.35300 \\
\hline$C$ & 3.517000 & -4.705200 & 0.58770 \\
\hline $\mathrm{H}$ & 3.769800 & -5.787100 & 0.65420 \\
\hline $\mathrm{H}$ & 2.977000 & -4.444700 & 1.52536 \\
\hline$C$ & 0.140300 & 1.038200 & 3.54270 \\
\hline $\mathrm{H}$ & -0.762600 & 1.534100 & 3.95420 \\
\hline $\mathrm{H}$ & 0.989100 & 1.314800 & 4.2080 \\
\hline $\mathrm{H}$ & 0.290000 & 1.463000 & 2.5309 \\
\hline 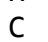 & 1.492200 & -1.787900 & 3.3422 \\
\hline
\end{tabular}




\begin{tabular}{|c|c|c|c|}
\hline $\mathrm{H}$ & 1.798700 & -1.880000 & 2.279100 \\
\hline $\mathrm{H}$ & 2.320200 & -1.332400 & 3.930900 \\
\hline $\mathrm{H}$ & 1.386200 & -2.827700 & 3.716400 \\
\hline C & -0.669400 & -1.132000 & 5.385400 \\
\hline $\mathrm{H}$ & -0.746400 & -2.197200 & 5.675200 \\
\hline $\mathrm{H}$ & 0.092100 & -0.657500 & 6.042500 \\
\hline $\mathrm{H}$ & -1.641900 & -0.644600 & 5.604700 \\
\hline C & -3.524900 & -3.701900 & 1.688300 \\
\hline $\mathrm{H}$ & -3.685200 & -3.596100 & 0.597100 \\
\hline $\mathrm{H}$ & -3.703000 & -4.774800 & 1.914000 \\
\hline $\mathrm{H}$ & -4.289000 & -3.122400 & 2.250400 \\
\hline C & -0.544100 & -4.226900 & 1.137600 \\
\hline $\mathrm{H}$ & 0.494100 & -4.151800 & 1.519200 \\
\hline $\mathrm{H}$ & -0.822300 & -5.302300 & 1.135300 \\
\hline $\mathrm{H}$ & -0.558500 & -3.867700 & 0.089300 \\
\hline C & -1.650300 & -4.070400 & 3.959200 \\
\hline $\mathrm{H}$ & -2.341000 & -3.600300 & 4.689600 \\
\hline $\mathrm{H}$ & -1.945600 & -5.137500 & 3.859700 \\
\hline $\mathrm{H}$ & -0.631300 & -4.055800 & 4.398100 \\
\hline C & -1.467800 & -1.357300 & 2.253600 \\
\hline C & -2.724300 & -0.702100 & 2.819600 \\
\hline $\mathrm{H}$ & -3.265600 & -1.111400 & 3.693100 \\
\hline C & -3.118800 & 0.516500 & 2.327500 \\
\hline H & -3.976600 & 1.031900 & 2.800700 \\
\hline C & -2.954400 & 2.505100 & 0.956000 \\
\hline C & -4.381600 & 2.487400 & 0.321500 \\
\hline $\mathrm{H}$ & -5.094400 & 1.986600 & 1.018800 \\
\hline $\mathrm{H}$ & -4.331900 & 1.891100 & -0.618500 \\
\hline C & -3.005700 & 3.399000 & 2.236300 \\
\hline $\mathrm{H}$ & -1.986900 & 3.431700 & 2.681000 \\
\hline H & -3.673100 & 2.954100 & 3.004200 \\
\hline C & -2.005100 & 3.208300 & -0.039100 \\
\hline $\mathrm{H}$ & -0.991800 & 3.255700 & 0.410000 \\
\hline $\mathrm{H}$ & -1.919800 & 2.594900 & -0.958500 \\
\hline C & -4.885100 & 3.913500 & 0.004000 \\
\hline H & -5.906800 & 3.859000 & -0.436200 \\
\hline C & -4.924200 & 4.747200 & 1.303900 \\
\hline $\mathrm{H}$ & -5.308200 & 5.769600 & 1.090100 \\
\hline $\mathrm{H}$ & -5.628000 & 4.287200 & 2.034200 \\
\hline C & -3.504100 & 4.826300 & 1.910600 \\
\hline $\mathrm{H}$ & -3.534800 & 5.422900 & 2.849700 \\
\hline C & -2.547900 & 5.490700 & 0.898400 \\
\hline $\mathrm{H}$ & -2.888600 & 6.524300 & 0.664100 \\
\hline $\mathrm{H}$ & -1.529200 & 5.578400 & 1.336800 \\
\hline C & -2.502200 & 4.633100 & -0.383600 \\
\hline $\mathrm{H}$ & -1.801900 & 5.089500 & -1.113800 \\
\hline C & -3.915400 & 4.567600 & -1.003800 \\
\hline $\mathrm{H}$ & -4.267500 & 5.589900 & -1.268400 \\
\hline $\mathrm{H}$ & -3.892400 & 3.980500 & -1.948200 \\
\hline N & 3.137400 & -0.626000 & -0.928800 \\
\hline N & -2.486300 & 1.124500 & 1.249400 \\
\hline 0 & 0.521300 & 0.456000 & 0.193100 \\
\hline $\mathrm{Si}$ & 2.275700 & 3.775800 & -1.104900 \\
\hline Si & 3.217000 & 2.144500 & -3.694400 \\
\hline Si & -0.169500 & -0.851400 & 3.561200 \\
\hline $\mathrm{Si}$ & -1.738100 & -3.248700 & 2.231700 \\
\hline K & 2.789100 & 0.711900 & 1.770900 \\
\hline K & -4.380400 & -0.971400 & 0.104700 \\
\hline C & -0.638600 & 1.339000 & -3.135100 \\
\hline C & -2.047000 & 1.224900 & -2.856500 \\
\hline C & 0.142000 & 0.149200 & -3.067800 \\
\hline C & -2.590700 & 0.140400 & -2.201500 \\
\hline $\mathrm{H}$ & -3.671900 & -0.063000 & -2.352900 \\
\hline C & -0.386200 & -0.952800 & -2.339200 \\
\hline C & -1.696700 & -0.833100 & -1.453100 \\
\hline $\mathrm{H}$ & -2.717100 & 1.978100 & -3.310200 \\
\hline $\mathrm{H}$ & -0.241900 & 2.238200 & -3.619100 \\
\hline H & 0.953000 & -0.013600 & -3.803100 \\
\hline
\end{tabular}




$\begin{array}{lrrr}\text { C } & -0.646800 & -2.367600 & -2.582100 \\ \text { C } & -1.871500 & -2.318500 & -1.835000 \\ \text { C } & -2.754600 & -3.396700 & -1.858200 \\ \text { H } & -3.754700 & -3.383500 & -1.389100 \\ \text { C } & -0.240000 & -3.527800 & -3.266100 \\ \text { C } & -1.118800 & -4.634800 & -3.225900 \\ \text { C } & -2.356300 & -4.571900 & -2.555800 \\ \text { H } & -3.030900 & -5.442900 & -2.579400 \\ \text { H } & 0.695200 & -3.577300 & -3.846300 \\ \text { H } & -0.842800 & -5.561700 & -3.755100 \\ \mathrm{C} & -7.831600 & -0.670500 & -0.149600 \\ \mathrm{C} & -7.668400 & -2.056800 & 0.044600 \\ \mathrm{C} & -7.080400 & -2.845400 & -0.965000 \\ \mathrm{C} & -6.654200 & -2.247700 & -2.167900 \\ \mathrm{C} & -6.818100 & -0.861600 & -2.361900 \\ \mathrm{C} & -7.407500 & -0.073200 & -1.353700 \\ \mathrm{H} & -8.300600 & -0.055500 & 0.635000 \\ \mathrm{H} & -8.009400 & -2.526400 & 0.981100 \\ \mathrm{H} & -6.959700 & -3.930100 & -0.815700 \\ \mathrm{H} & -6.194300 & -2.862000 & -2.957700 \\ \mathrm{H} & -6.489100 & -0.396300 & -3.304500 \\ \mathrm{H} & -7.541700 & 1.008900 & -1.509200 \\ \mathrm{H} & 3.754800 & 3.481200 & 4.796700 \\ \mathrm{C} & 4.332600 & 2.615300 & 4.436600 \\ \mathrm{C} & 5.187400 & 2.752400 & 3.325300 \\ \mathrm{C} & 4.222200 & 1.372900 & 5.091500 \\ \mathrm{C} & 5.931000 & 1.646600 & 2.867300 \\ \mathrm{H} & 5.279600 & 3.725200 & 2.817100 \\ \mathrm{C} & 4.966300 & 0.267400 & 4.634400 \\ \mathrm{H} & 3.560100 & 1.267500 & 5.965500 \\ \mathrm{C} & 5.820500 & 0.404100 & 3.522300 \\ \mathrm{H} & 6.601300 & 1.755900 & 2.000400 \\ \mathrm{H} & 4.888100 & -0.701600 & 5.152400 \\ \mathrm{H} & 6.407300 & -0.458600 & 3.169300\end{array}$

\section{Table S19. Cartesian coordinates of Int-6}

Zero-point correction=

Thermal correction to Energy=

Thermal correction to Enthalpy=

Thermal correction to Gibbs Free Energy=

Sum of electronic and zero-point Energies $=\quad-5441.550569$

Sum of electronic and thermal Energies $=\quad-5441.465218$

Sum of electronic and thermal Enthalpies $=\quad-5441.464274$

Sum of electronic and thermal Free Energies $=-5441.675483$
1.330859 (Hartree/Particle)

1.416209

1.417153

1.205944

$\begin{array}{lrrr}\text { Al } & 1.521700 & 0.181000 & -1.194000 \\ \text { Al } & -1.106400 & -0.008000 & 0.463600 \\ \text { C } & 0.789200 & 4.109800 & -2.441300 \\ \text { H } & -0.018100 & 3.351500 & -2.482900 \\ \text { H } & 0.380600 & 5.037400 & -1.987900 \\ \text { H } & 1.074800 & 4.349100 & -3.483800 \\ \text { C } & 1.847400 & 3.741500 & 0.416100 \\ \text { H } & 2.752200 & 3.698900 & 1.062700 \\ \text { H } & 1.402900 & 4.747000 & 0.574600 \\ \text { H } & 1.102600 & 2.982900 & 0.736900 \\ \text { C } & 3.666300 & 4.805400 & -1.729000 \\ \text { H } & 3.892500 & 4.941500 & -2.806800 \\ \text { H } & 3.343700 & 5.792700 & -1.331800 \\ \text { H } & 4.614000 & 4.534000 & -1.217500 \\ \text { C } & 2.485500 & 2.561600 & -4.986000 \\ \text { H } & 2.677100 & 3.653100 & -4.919100 \\ \text { H } & 2.840600 & 2.228900 & -5.985700\end{array}$




\begin{tabular}{|c|c|c|c|}
\hline $\mathrm{H}$ & 1.390200 & 2.404600 & -4.942200 \\
\hline C & 3.609400 & -0.203600 & -4.244600 \\
\hline $\mathrm{H}$ & 2.645900 & -0.750500 & -4.284300 \\
\hline $\mathrm{H}$ & 4.047200 & -0.218000 & -5.266100 \\
\hline $\mathrm{H}$ & 4.289700 & -0.767200 & -3.577100 \\
\hline C & 5.247500 & 2.264000 & -3.813600 \\
\hline $\mathrm{H}$ & 5.970800 & 1.637300 & -3.253100 \\
\hline $\mathrm{H}$ & 5.540100 & 2.238400 & -4.885900 \\
\hline $\mathrm{H}$ & 5.357900 & 3.309700 & -3.462500 \\
\hline C & 2.805400 & 1.729700 & -1.830700 \\
\hline C & 4.053200 & 1.311300 & -1.056200 \\
\hline $\mathrm{H}$ & 4.938400 & 1.968300 & -0.970100 \\
\hline C & 4.174200 & 0.012100 & -0.618000 \\
\hline $\mathrm{H}$ & 5.142500 & -0.331800 & -0.204200 \\
\hline C & 3.424100 & -2.313300 & -0.483600 \\
\hline C & 2.118800 & -3.123100 & -0.318600 \\
\hline $\mathrm{H}$ & 1.465300 & -2.943500 & -1.196900 \\
\hline $\mathrm{H}$ & 1.566800 & -2.743800 & 0.568600 \\
\hline C & 4.176400 & -2.876700 & -1.728100 \\
\hline $\mathrm{H}$ & 5.121000 & -2.306800 & -1.874300 \\
\hline $\mathrm{H}$ & 3.551100 & -2.697900 & -2.627800 \\
\hline C & 4.312500 & -2.599200 & 0.769400 \\
\hline $\mathrm{H}$ & 3.790100 & -2.223800 & 1.678600 \\
\hline $\mathrm{H}$ & 5.277100 & -2.051700 & 0.697500 \\
\hline C & 2.403000 & -4.636700 & -0.170600 \\
\hline $\mathrm{H}$ & 1.437200 & -5.177200 & -0.062800 \\
\hline C & 3.144300 & -5.151000 & -1.425200 \\
\hline $\mathrm{H}$ & 2.510900 & -5.004200 & -2.326900 \\
\hline $\mathrm{H}$ & 3.334800 & -6.244200 & -1.336400 \\
\hline C & 4.476600 & -4.384800 & -1.580800 \\
\hline $\mathrm{H}$ & 5.011500 & -4.744100 & -2.488200 \\
\hline C & 5.357200 & -4.616200 & -0.333600 \\
\hline $\mathrm{H}$ & 5.597600 & -5.697500 & -0.224400 \\
\hline $\mathrm{H}$ & 6.327300 & -4.081300 & -0.444400 \\
\hline C & 4.609300 & -4.110100 & 0.919900 \\
\hline $\mathrm{H}$ & 5.242600 & -4.268000 & 1.821800 \\
\hline C & 3.282500 & -4.882700 & 1.075700 \\
\hline $\mathrm{H}$ & 3.483000 & -5.970900 & 1.197600 \\
\hline $\mathrm{H}$ & 2.751200 & -4.549500 & 1.994200 \\
\hline C & -0.171800 & 1.248600 & 3.623500 \\
\hline $\mathrm{H}$ & -1.127400 & 1.697300 & 3.961300 \\
\hline $\mathrm{H}$ & 0.603200 & 1.525300 & 4.373000 \\
\hline $\mathrm{H}$ & 0.073500 & 1.712200 & 2.647200 \\
\hline C & 1.416200 & -1.383400 & 3.336600 \\
\hline $\mathrm{H}$ & 1.711300 & -1.435900 & 2.269000 \\
\hline $\mathrm{H}$ & 2.163500 & -0.795300 & 3.915600 \\
\hline $\mathrm{H}$ & 1.466200 & -2.415600 & 3.733600 \\
\hline C & -0.827200 & -1.115000 & 5.339400 \\
\hline $\mathrm{H}$ & -0.750200 & -2.203500 & 5.540600 \\
\hline $\mathrm{H}$ & -0.135100 & -0.601600 & 6.042300 \\
\hline $\mathrm{H}$ & -1.857000 & -0.787400 & 5.590300 \\
\hline C & -2.859900 & -3.956800 & 0.959500 \\
\hline $\mathrm{H}$ & -2.419500 & -3.795500 & -0.044800 \\
\hline $\mathrm{H}$ & -2.819600 & -5.049800 & 1.156000 \\
\hline $\mathrm{H}$ & -3.947100 & -3.717700 & 0.937100 \\
\hline C & -0.342900 & -4.177500 & 2.595700 \\
\hline $\mathrm{H}$ & 0.111400 & -4.059500 & 3.600600 \\
\hline $\mathrm{H}$ & -0.625300 & -5.246100 & 2.488100 \\
\hline $\mathrm{H}$ & 0.434900 & -3.956800 & 1.839600 \\
\hline C & -3.009400 & -3.522900 & 3.889300 \\
\hline $\mathrm{H}$ & -4.053700 & -3.169900 & 3.753000 \\
\hline $\mathrm{H}$ & -3.049900 & -4.628400 & 4.000000 \\
\hline $\mathrm{H}$ & -2.638400 & -3.104400 & 4.844900 \\
\hline C & -1.603100 & -1.218000 & 2.206200 \\
\hline C & -2.881000 & -0.518800 & 2.671800 \\
\hline $\mathrm{H}$ & -3.470800 & -0.880700 & 3.532100 \\
\hline C & -3.217600 & 0.696800 & 2.127700 \\
\hline $\mathrm{H}$ & -4.083500 & 1.246700 & 2.543700 \\
\hline
\end{tabular}




\begin{tabular}{|c|c|c|c|}
\hline$C$ & -2.922900 & 2.659200 & 0.752200 \\
\hline C & -4.396600 & 2.722300 & 0.238200 \\
\hline $\mathrm{H}$ & -5.084000 & 2.301100 & 1.006100 \\
\hline $\mathrm{H}$ & -4.484100 & 2.082700 & -0.669600 \\
\hline C & -2.814500 & 3.591800 & 2.000700 \\
\hline $\mathrm{H}$ & -1.764700 & 3.571400 & 2.364300 \\
\hline $\mathrm{H}$ & -3.445700 & 3.202800 & 2.828800 \\
\hline C & -2.010500 & 3.261400 & -0.339300 \\
\hline $\mathrm{H}$ & -0.962700 & 3.251000 & 0.026000 \\
\hline $\mathrm{H}$ & -2.043500 & 2.621400 & -1.244100 \\
\hline C & -4.822300 & 4.169200 & -0.097800 \\
\hline $\mathrm{H}$ & -5.876400 & 4.170700 & -0.456800 \\
\hline C & -4.705200 & 5.045400 & 1.169800 \\
\hline $\mathrm{H}$ & -5.031100 & 6.085800 & 0.946400 \\
\hline $\mathrm{H}$ & -5.382500 & 4.661100 & 1.965600 \\
\hline C & -3.241800 & 5.039200 & 1.666200 \\
\hline $\mathrm{H}$ & -3.157500 & 5.665000 & 2.582700 \\
\hline C & -2.322500 & 5.602700 & 0.561900 \\
\hline $\mathrm{H}$ & -2.609000 & 6.650400 & 0.317600 \\
\hline $\mathrm{H}$ & -1.269400 & 5.630800 & 0.919200 \\
\hline$C$ & -2.434900 & 4.707900 & -0.690900 \\
\hline $\mathrm{H}$ & -1.763800 & 5.091100 & -1.487700 \\
\hline C & -3.893000 & 4.721900 & -1.199800 \\
\hline $\mathrm{H}$ & -4.195200 & 5.757200 & -1.474700 \\
\hline $\mathrm{H}$ & -3.982000 & 4.104600 & -2.121100 \\
\hline $\mathrm{N}$ & 3.106900 & -0.877600 & -0.649200 \\
\hline $\mathrm{N}$ & -2.517200 & 1.264300 & 1.075200 \\
\hline 0 & 0.589400 & 0.501100 & 0.328700 \\
\hline Si & 2.286100 & 3.510400 & -1.437100 \\
\hline Si & 3.458700 & 1.600200 & -3.647100 \\
\hline Si & -0.342000 & -0.653500 & 3.546800 \\
\hline $\mathrm{Si}$ & -1.899700 & -3.104000 & 2.383700 \\
\hline K & -4.199500 & -1.227000 & 0.027800 \\
\hline$C$ & -1.142700 & 1.031200 & -4.128800 \\
\hline C & -2.392800 & 1.078100 & -3.377300 \\
\hline$C$ & -0.076800 & 0.316100 & -3.675500 \\
\hline$C$ & -2.560600 & 0.378900 & -2.220400 \\
\hline $\mathrm{H}$ & -3.533600 & 0.525000 & -1.712000 \\
\hline $\mathrm{C}$ & -0.048800 & -0.471500 & -2.396400 \\
\hline$C$ & -1.506600 & -0.512300 & -1.602400 \\
\hline $\mathrm{H}$ & -3.212100 & 1.715500 & -3.752800 \\
\hline $\mathrm{H}$ & -1.071900 & 1.585900 & -5.078900 \\
\hline $\mathrm{H}$ & 0.832800 & 0.280400 & -4.297200 \\
\hline C & -0.314100 & -1.934600 & -2.724200 \\
\hline C & -1.598000 & -1.951100 & -2.123900 \\
\hline$C$ & -2.465800 & -3.033700 & -2.332700 \\
\hline $\mathrm{H}$ & -3.520300 & -3.054800 & -2.003100 \\
\hline C & 0.172700 & -3.007400 & -3.478000 \\
\hline C & -0.679000 & -4.129900 & -3.624500 \\
\hline$C$ & -1.975500 & -4.136300 & -3.078000 \\
\hline $\mathrm{H}$ & -2.630100 & -5.006400 & -3.250300 \\
\hline $\mathrm{H}$ & 1.153100 & -2.979900 & -3.981100 \\
\hline $\mathrm{H}$ & -0.337200 & -4.999400 & -4.209300 \\
\hline K & 2.759500 & 1.035400 & 1.839800 \\
\hline $\mathrm{H}$ & 3.297400 & 3.719100 & 4.965000 \\
\hline C & 4.017200 & 2.993500 & 4.553900 \\
\hline C & 4.126400 & 1.709500 & 5.123100 \\
\hline C & 4.834600 & 3.352900 & 3.464100 \\
\hline $\mathrm{C}$ & 5.053200 & 0.784600 & 4.602400 \\
\hline $\mathrm{H}$ & 3.493600 & 1.431900 & 5.981000 \\
\hline C & 5.759700 & 2.427400 & 2.941800 \\
\hline $\mathrm{H}$ & 4.754900 & 4.359300 & 3.023700 \\
\hline $\mathrm{H}$ & 5.144700 & -0.216600 & 5.052700 \\
\hline C & 5.869400 & 1.143700 & 3.512100 \\
\hline $\mathrm{H}$ & 6.398700 & 2.708000 & 2.090100 \\
\hline $\mathrm{H}$ & 6.597000 & 0.422600 & 3.107300 \\
\hline C & -7.141600 & -3.046200 & -0.162200 \\
\hline C & -7.552600 & -1.895300 & 0.539900 \\
\hline
\end{tabular}




$\begin{array}{lrrr}\text { C } & -7.593600 & -0.648000 & -0.115000 \\ \mathrm{C} & -7.221300 & -0.551300 & -1.470900 \\ \mathrm{C} & -6.807600 & -1.701600 & -2.172300 \\ \mathrm{C} & -6.769000 & -2.949100 & -1.517900 \\ \mathrm{H} & -7.120000 & -4.023200 & 0.346300 \\ \mathrm{H} & -7.851600 & -1.972400 & 1.597400 \\ \mathrm{H} & -7.923800 & 0.250200 & 0.430500 \\ \mathrm{H} & -7.259900 & 0.422200 & -1.984700 \\ \mathrm{H} & -6.518700 & -1.626500 & -3.232500 \\ \mathrm{H} & -6.455500 & -3.849800 & -2.069100\end{array}$

Table S20. Cartesian coordinates of TS-5

Zero-point correction= 1.328990 (Hartree/Particle)

Thermal correction to Energy= 1.414170

Thermal correction to Enthalpy=

Thermal correction to Gibbs Free Energy=

Sum of electronic and zero-point Energies $=\quad-5441.545453$

Sum of electronic and thermal Energies $=\quad-5441.460272$

Sum of electronic and thermal Enthalpies $=\quad-5441.459328$

Sum of electronic and thermal Free Energies $=-5441.670059$

$\begin{array}{lrrr}\text { Al } & 2.044200 & 0.410800 & -1.244100 \\ \text { Al } & -1.019300 & -0.090600 & 0.010500 \\ \text { C } & 1.434500 & 4.711000 & -1.524000 \\ \text { H } & 0.628000 & 4.069400 & -1.932800 \\ \text { H } & 0.968700 & 5.523100 & -0.926600 \\ \text { H } & 1.957900 & 5.188100 & -2.378200 \\ \mathrm{C} & 1.799500 & 3.355100 & 1.226900 \\ \text { H } & 2.552100 & 3.122800 & 2.013500 \\ \text { H } & 1.249400 & 4.250900 & 1.585800 \\ \text { H } & 1.076800 & 2.519000 & 1.122300 \\ \mathrm{C} & 4.032600 & 4.951500 & 0.050900 \\ \mathrm{H} & 4.527500 & 5.435000 & -0.814300 \\ \mathrm{H} & 3.590000 & 5.757500 & 0.676600 \\ \mathrm{H} & 4.823500 & 4.460500 & 0.656700 \\ \mathrm{C} & 2.899000 & 3.055300 & -4.410000 \\ \mathrm{H} & 2.220100 & 3.877500 & -4.109800 \\ \mathrm{H} & 3.534800 & 3.434200 & -5.239800 \\ \mathrm{H} & 2.282100 & 2.232400 & -4.823000 \\ \mathrm{C} & 4.923800 & 0.914800 & -3.706300 \\ \mathrm{H} & 4.221900 & 0.076300 & -3.886800 \\ \mathrm{H} & 5.397900 & 1.173800 & -4.677300 \\ \mathrm{H} & 5.717100 & 0.554100 & -3.021900 \\ \mathrm{C} & 5.432400 & 3.788700 & -2.927600 \\ \mathrm{H} & 6.131700 & 3.625600 & -2.082200 \\ \mathrm{H} & 6.027100 & 3.755200 & -3.866200 \\ \mathrm{H} & 5.018000 & 4.814100 & -2.837000 \\ \mathrm{C} & 3.275400 & 2.127900 & -1.271600 \\ \mathrm{C} & 4.470300 & 1.601300 & -0.481100 \\ \mathrm{H} & 5.298000 & 2.260400 & -0.159900 \\ \mathrm{C} & 4.614600 & 0.249200 & -0.287800 \\ \mathrm{H} & 5.548800 & -0.139800 & 0.163300 \\ \mathrm{C} & 4.002200 & -2.073500 & -0.725600 \\ \mathrm{C} & 2.753300 & -2.945500 & -0.970800 \\ \mathrm{H} & 2.237300 & -2.592500 & -1.887400 \\ \mathrm{H} & 2.033500 & -2.794100 & -0.140000 \\ \mathrm{C} & 4.983200 & -2.310900 & -1.913800 \\ \mathrm{H} & 5.896500 & -1.692200 & -1.770800 \\ \mathrm{H} & 4.499700 & -1.949100 & -2.845900 \\ \mathrm{C} & 4.694300 & -2.605300 & 0.570900 \\ \mathrm{H} & 4.004000 & -2.471500 & 1.435500 \\ \mathrm{H} & 5.611000 & -2.016600 & 0.792200\end{array}$




\begin{tabular}{|c|c|c|c|}
\hline C & 3.116100 & -4.441300 & -1.105100 \\
\hline $\mathrm{H}$ & 2.185000 & -5.022800 & -1.280600 \\
\hline C & 4.083700 & -4.634400 & -2.293800 \\
\hline $\mathrm{H}$ & 3.596100 & -4.314600 & -3.241000 \\
\hline $\mathrm{H}$ & 4.339600 & -5.711600 & -2.411100 \\
\hline C & 5.362700 & -3.802800 & -2.050000 \\
\hline $\mathrm{H}$ & 6.061300 & -3.928000 & -2.907200 \\
\hline C & 6.046800 & -4.280100 & -0.749600 \\
\hline $\mathrm{H}$ & 6.344100 & -5.349000 & -0.838200 \\
\hline $\mathrm{H}$ & 6.980900 & -3.701000 & -0.572600 \\
\hline C & 5.074600 & -4.098500 & 0.437900 \\
\hline $\mathrm{H}$ & 5.567100 & -4.435500 & 1.377900 \\
\hline C & 3.798000 & -4.930600 & 0.192400 \\
\hline $\mathrm{H}$ & 4.052200 & -6.011300 & 0.112000 \\
\hline $\mathrm{H}$ & 3.102000 & -4.827400 & 1.054700 \\
\hline C & -0.124500 & 0.401100 & 3.433300 \\
\hline $\mathrm{H}$ & -1.055200 & 0.814400 & 3.872300 \\
\hline $\mathrm{H}$ & 0.658300 & 0.480900 & 4.221200 \\
\hline $\mathrm{H}$ & 0.125300 & 1.058200 & 2.577400 \\
\hline C & 1.198900 & -2.369700 & 2.762900 \\
\hline $\mathrm{H}$ & 1.649600 & -2.220700 & 1.760300 \\
\hline $\mathrm{H}$ & 1.939500 & -2.106400 & 3.551500 \\
\hline $\mathrm{H}$ & 1.019800 & -3.460900 & 2.862500 \\
\hline C & -1.172700 & -2.100300 & 4.614500 \\
\hline $\mathrm{H}$ & -1.335600 & -3.195100 & 4.617800 \\
\hline $\mathrm{H}$ & -0.451800 & -1.867500 & 5.429100 \\
\hline $\mathrm{H}$ & -2.131700 & -1.608400 & 4.877800 \\
\hline C & -3.545400 & -3.660000 & 0.002500 \\
\hline $\mathrm{H}$ & -3.606200 & -3.107900 & -0.957700 \\
\hline $\mathrm{H}$ & -3.604300 & -4.736900 & -0.263800 \\
\hline $\mathrm{H}$ & -4.433900 & -3.458100 & 0.641700 \\
\hline C & -0.521000 & -4.132300 & -0.099400 \\
\hline $\mathrm{H}$ & 0.411600 & -4.236900 & 0.490100 \\
\hline $\mathrm{H}$ & -0.810000 & -5.144900 & -0.452800 \\
\hline $\mathrm{H}$ & -0.299100 & -3.500500 & -0.983200 \\
\hline C & -2.134700 & -4.590600 & 2.432400 \\
\hline $\mathrm{H}$ & -2.919400 & -4.253400 & 3.142000 \\
\hline $\mathrm{H}$ & -2.446300 & -5.582600 & 2.038500 \\
\hline $\mathrm{H}$ & -1.202400 & -4.750000 & 3.012900 \\
\hline C & -1.609500 & -1.570000 & 1.448700 \\
\hline C & -2.861500 & -0.925400 & 2.038600 \\
\hline $\mathrm{H}$ & -3.471800 & -1.417200 & 2.821200 \\
\hline C & -3.096500 & 0.407500 & 1.778900 \\
\hline $\mathrm{H}$ & -3.923400 & 0.918900 & 2.312900 \\
\hline C & -2.777600 & 2.533100 & 0.654100 \\
\hline C & -4.136700 & 2.574900 & -0.115400 \\
\hline $\mathrm{H}$ & -4.916000 & 2.051100 & 0.488600 \\
\hline $\mathrm{H}$ & -4.014700 & 2.022700 & -1.075600 \\
\hline C & -2.954200 & 3.336500 & 1.980900 \\
\hline $\mathrm{H}$ & -1.986700 & 3.325300 & 2.529000 \\
\hline $\mathrm{H}$ & -3.700200 & 2.845700 & 2.642800 \\
\hline C & -1.732400 & 3.292200 & -0.193300 \\
\hline $\mathrm{H}$ & -0.770000 & 3.298800 & 0.356500 \\
\hline $\mathrm{H}$ & -1.548100 & 2.739200 & -1.136800 \\
\hline C & -4.597000 & 4.023700 & -0.392400 \\
\hline $\mathrm{H}$ & -5.569400 & 4.009400 & -0.935100 \\
\hline C & -4.761200 & 4.770600 & 0.950500 \\
\hline $\mathrm{H}$ & -5.112200 & 5.810600 & 0.767800 \\
\hline $\mathrm{H}$ & -5.540900 & 4.275100 & 1.572800 \\
\hline C & -3.410400 & 4.788600 & 1.703300 \\
\hline $\mathrm{H}$ & -3.533000 & 5.323100 & 2.671700 \\
\hline C & -2.354800 & 5.508700 & 0.837800 \\
\hline $\mathrm{H}$ & -2.667200 & 6.559200 & 0.643300 \\
\hline $\mathrm{H}$ & -1.384200 & 5.556500 & 1.378900 \\
\hline C & -2.184900 & 4.740700 & -0.490400 \\
\hline $\mathrm{H}$ & -1.411200 & 5.236900 & -1.112800 \\
\hline C & -3.527800 & 4.730500 & -1.253200 \\
\hline o & -3.848500 & 5.770600 & -1.485100 \\
\hline
\end{tabular}




\begin{tabular}{rrr}
-3.412700 & 4.205800 & -2.227600 \\
3.605200 & -0.651400 & -0.618100 \\
-2.336800 & 1.137800 & 0.884900 \\
0.727000 & 0.308200 & -0.007800 \\
2.653100 & 3.720500 & -0.449700 \\
4.056200 & 2.459400 & -3.008700 \\
-0.458400 & -1.429200 & 2.969800 \\
-1.904300 & -3.386500 & 0.957200 \\
2.692200 & 0.348000 & 2.009600 \\
-4.960700 & -0.865500 & -0.157400 \\
1.190900 & -0.041600 & -3.063100 \\
0.196800 & 1.089200 & -3.206000 \\
0.493700 & -1.361400 & -3.156200 \\
-1.085000 & 0.991300 & -2.761200 \\
-1.806300 & 1.799400 & -2.972700 \\
-0.766400 & -1.466400 & -2.654800 \\
-1.539800 & -0.257100 & -2.058600 \\
0.526800 & 2.006400 & -3.718100 \\
1.999800 & 0.035900 & -3.818100 \\
0.938100 & -2.170100 & -3.762700 \\
-2.033700 & -2.143400 & -2.979300 \\
-2.774000 & -0.976000 & -2.593200 \\
-4.113000 & -0.825700 & -2.973100 \\
-4.661000 & 0.130900 & -2.868000 \\
-2.645200 & -3.243500 & -3.593500 \\
-4.030200 & -3.127000 & -3.873400 \\
-4.743100 & -1.940700 & -3.598800 \\
-5.795200 & -1.860800 & -3.920100 \\
-2.087000 & -4.144900 & -3.893400 \\
-4.554000 & -3.963300 & -4.364600 \\
-7.882700 & -0.255900 & 1.618200 \\
-7.733800 & -1.650900 & 1.750400 \\
-7.911400 & -2.487700 & 0.630600 \\
-8.237000 & -1.929500 & -0.621700 \\
-8.385800 & -0.534400 & -0.753900 \\
-8.209000 & 0.302500 & 0.366100 \\
-7.752800 & 0.397200 & 2.495600 \\
-7.485800 & -2.087900 & 2.730700 \\
-7.802700 & -3.578800 & 0.735000 \\
-8.381500 & -2.584500 & -1.495300 \\
-8.650700 & -0.099200 & -1.730700 \\
-8.336400 & 1.392000 & 0.265500 \\
2.988500 & 2.375700 & 5.722600 \\
3.685200 & 1.660700 & 5.256900 \\
4.649000 & 2.115100 & 4.335600 \\
3.616000 & 0.293000 & 5.587400 \\
5.543900 & 1.201900 & 3.743600 \\
4.707600 & 3.185000 & 4.080900 \\
4.512000 & -0.620100 & 4.997300 \\
2.866800 & -0.061300 & 6.313000 \\
5.475600 & -0.165700 & 4.075700 \\
6.297400 & 1.557600 & 3.023700 \\
4.464500 & -1.688300 & 5.262300 \\
\hline .178500 & -0.879000 & 3.617200
\end{tabular}

Table S21. Cartesian coordinates of $7 \cdot \mathrm{C}_{6} \mathrm{H}_{6}$ Zero-point correction=

Thermal correction to Energy=

1.329216 (Hartree/Particle)

Thermal correction to Enthalpy=

1.415129

1.416073

1.203067

Thermal correction to Gibbs Free Energy=

Sum of electronic and zero-point Energies $=-5441.575693$

Sum of electronic and thermal Energies= $\quad-5441.489780$

Sum of electronic and thermal Enthalpies $=\quad-5441.488836$ 


\begin{tabular}{|c|c|c|c|}
\hline Al & 1.671900 & 0.385900 & -1.278000 \\
\hline $\mathrm{Al}$ & -1.198600 & -0.272200 & 0.380700 \\
\hline C & 0.669200 & 4.421700 & -1.963100 \\
\hline $\mathrm{H}$ & -0.085100 & 3.627000 & -2.128300 \\
\hline $\mathrm{H}$ & 0.177000 & 5.268700 & -1.440600 \\
\hline $\mathrm{H}$ & 0.996800 & 4.787400 & -2.955500 \\
\hline $\mathrm{C}$ & 1.546900 & 3.681200 & 0.886600 \\
\hline $\mathrm{H}$ & 2.404100 & 3.550000 & 1.584100 \\
\hline $\mathrm{H}$ & 1.042600 & 4.624000 & 1.187900 \\
\hline $\mathrm{H}$ & 0.820300 & 2.851300 & 1.015700 \\
\hline$C$ & 3.412900 & 5.214900 & -0.917700 \\
\hline $\mathrm{H}$ & 3.716000 & 5.516600 & -1.941500 \\
\hline $\mathrm{H}$ & 2.972800 & 6.109400 & -0.425200 \\
\hline $\mathrm{H}$ & 4.335600 & 4.947700 & -0.360500 \\
\hline $\mathrm{C}$ & 2.583900 & 3.296500 & -4.601000 \\
\hline $\mathrm{H}$ & 2.593500 & 4.382900 & -4.375100 \\
\hline $\mathrm{H}$ & 3.073700 & 3.164000 & -5.590300 \\
\hline $\mathrm{H}$ & 1.529300 & 2.975000 & -4.710900 \\
\hline $\mathrm{C}$ & 3.883300 & 0.581100 & -4.112100 \\
\hline $\mathrm{H}$ & 2.931000 & 0.048000 & -4.307700 \\
\hline $\mathrm{H}$ & 4.419800 & 0.710000 & -5.076900 \\
\hline $\mathrm{H}$ & 4.508600 & -0.052500 & -3.453700 \\
\hline$C$ & 5.291700 & 3.127600 & -3.294400 \\
\hline $\mathrm{H}$ & 6.048200 & 2.508700 & -2.770100 \\
\hline $\mathrm{H}$ & 5.638300 & 3.265300 & -4.341700 \\
\hline $\mathrm{H}$ & 5.278400 & 4.127000 & -2.814000 \\
\hline $\mathrm{C}$ & 2.826400 & 2.134100 & -1.535000 \\
\hline$C$ & 4.064600 & 1.736700 & -0.736900 \\
\hline $\mathrm{H}$ & 4.871700 & 2.456600 & -0.507900 \\
\hline $\mathrm{C}$ & 4.281700 & 0.412000 & -0.441500 \\
\hline $\mathrm{H}$ & 5.248000 & 0.106100 & 0.005800 \\
\hline$C$ & 3.750000 & -1.966500 & -0.583200 \\
\hline $\mathrm{C}$ & 2.527900 & -2.903200 & -0.683400 \\
\hline $\mathrm{H}$ & 1.976900 & -2.686900 & -1.621200 \\
\hline $\mathrm{H}$ & 1.829000 & -2.678700 & 0.150500 \\
\hline $\mathrm{C}$ & 4.718300 & -2.315500 & -1.754900 \\
\hline $\mathrm{H}$ & 5.609100 & -1.650300 & -1.707900 \\
\hline $\mathrm{H}$ & 4.202500 & -2.094100 & -2.712800 \\
\hline$C$ & 4.486300 & -2.309500 & 0.752700 \\
\hline $\mathrm{H}$ & 3.807600 & -2.093100 & 1.609600 \\
\hline $\mathrm{H}$ & 5.385500 & -1.667900 & 0.877700 \\
\hline$C$ & 2.945700 & -4.390800 & -0.649100 \\
\hline $\mathrm{H}$ & 2.034700 & -5.021200 & -0.735800 \\
\hline$C$ & 3.899200 & -4.692300 & -1.827800 \\
\hline $\mathrm{H}$ & 3.380600 & -4.502900 & -2.792900 \\
\hline $\mathrm{H}$ & 4.188800 & -5.767300 & -1.821400 \\
\hline$C$ & 5.152800 & -3.797200 & -1.712700 \\
\hline $\mathrm{H}$ & 5.841100 & -4.003800 & -2.562500 \\
\hline$C$ & 5.875000 & -4.087400 & -0.378800 \\
\hline $\mathrm{H}$ & 6.210500 & -5.148100 & -0.343400 \\
\hline $\mathrm{H}$ & 6.790000 & -3.458700 & -0.294200 \\
\hline$C$ & 4.916600 & -3.795500 & 0.797600 \\
\hline $\mathrm{H}$ & 5.436400 & -3.996100 & 1.761700 \\
\hline$C$ & 3.667700 & -4.696700 & 0.680400 \\
\hline $\mathrm{H}$ & 3.963300 & -5.769100 & 0.724500 \\
\hline $\mathrm{H}$ & 2.985100 & -4.516300 & 1.540400 \\
\hline $\mathrm{C}$ & -0.189200 & 0.861200 & 3.588200 \\
\hline $\mathrm{H}$ & -1.123700 & 1.292800 & 4.001000 \\
\hline $\mathrm{H}$ & 0.627800 & 1.134300 & 4.293300 \\
\hline $\mathrm{H}$ & -0.029600 & 1.359600 & 2.611200 \\
\hline$C$ & 1.294300 & -1.887000 & 3.289000 \\
\hline $\mathrm{H}$ & 1.640400 & -1.898100 & 2.234400 \\
\hline $\mathrm{H}$ & 2.075600 & -1.428900 & 3.936300 \\
\hline $\mathrm{H}$ & 1.221700 & -2.949700 & 3.601600 \\
\hline$C$ & -0.991800 & -1.463100 & 5.248800 \\
\hline
\end{tabular}




\begin{tabular}{|c|c|c|c|}
\hline $\mathrm{H}$ & -1.035900 & -2.549800 & 5.456200 \\
\hline $\mathrm{H}$ & -0.279600 & -1.011900 & 5.974200 \\
\hline $\mathrm{H}$ & -1.992500 & -1.032700 & 5.460400 \\
\hline C & -3.451600 & -3.836600 & 0.966200 \\
\hline $\mathrm{H}$ & -3.437400 & -3.518200 & -0.096800 \\
\hline $\mathrm{H}$ & -3.556900 & -4.942200 & 0.955500 \\
\hline $\mathrm{H}$ & -4.351900 & -3.437000 & 1.482500 \\
\hline C & -0.430700 & -4.284800 & 0.993600 \\
\hline $\mathrm{H}$ & 0.531600 & -4.187500 & 1.534700 \\
\hline $\mathrm{H}$ & -0.665700 & -5.368800 & 0.927200 \\
\hline $\mathrm{H}$ & -0.287300 & -3.904300 & -0.037500 \\
\hline C & -2.059200 & -4.295400 & 3.561800 \\
\hline H & -2.838000 & -3.830800 & 4.202000 \\
\hline $\mathrm{H}$ & -2.366900 & -5.345700 & 3.367500 \\
\hline $\mathrm{H}$ & -1.119300 & -4.334800 & 4.150100 \\
\hline C & -1.611600 & -1.500500 & 2.069200 \\
\hline C & -2.907600 & -0.869700 & 2.571500 \\
\hline $\mathrm{H}$ & -3.481000 & -1.288500 & 3.420300 \\
\hline C & -3.279700 & 0.361700 & 2.087600 \\
\hline $\mathrm{H}$ & -4.145200 & 0.877300 & 2.549500 \\
\hline C & -3.057500 & 2.378100 & 0.769200 \\
\hline C & -4.561200 & 2.437800 & 0.341700 \\
\hline H & -5.202100 & 1.981600 & 1.129700 \\
\hline $\mathrm{H}$ & -4.691200 & 1.836200 & -0.589100 \\
\hline C & -2.885700 & 3.282300 & 2.030700 \\
\hline $\mathrm{H}$ & -1.814800 & 3.267500 & 2.327000 \\
\hline $\mathrm{H}$ & -3.458300 & 2.858000 & 2.883800 \\
\hline C & -2.227600 & 3.020800 & -0.366000 \\
\hline $\mathrm{H}$ & -1.156800 & 3.006100 & -0.076800 \\
\hline $\mathrm{H}$ & -2.309900 & 2.409200 & -1.288400 \\
\hline C & -5.027900 & 3.886500 & 0.076300 \\
\hline $\mathrm{H}$ & -6.102700 & 3.880800 & -0.215400 \\
\hline C & -4.843600 & 4.727300 & 1.359900 \\
\hline $\mathrm{H}$ & -5.200000 & 5.767400 & 1.188800 \\
\hline $\mathrm{H}$ & -5.462700 & 4.308200 & 2.185100 \\
\hline C & -3.352000 & 4.730800 & 1.762300 \\
\hline $\mathrm{H}$ & -3.217700 & 5.335400 & 2.686900 \\
\hline C & -2.511500 & 5.334000 & 0.616800 \\
\hline $\mathrm{H}$ & -2.824600 & 6.383500 & 0.417800 \\
\hline $\mathrm{H}$ & -1.438700 & 5.367400 & 0.907900 \\
\hline C & -2.690700 & 4.471000 & -0.650600 \\
\hline $\mathrm{H}$ & -2.074900 & 4.884700 & -1.475500 \\
\hline C & -4.177600 & 4.481300 & -1.066300 \\
\hline $\mathrm{H}$ & -4.507000 & 5.520000 & -1.291900 \\
\hline $\mathrm{H}$ & -4.319900 & 3.889300 & -1.997900 \\
\hline $\mathrm{N}$ & 3.305600 & -0.556300 & -0.650600 \\
\hline $\mathrm{N}$ & -2.619800 & 0.983700 & 1.033800 \\
\hline 0 & 0.477900 & 0.288600 & 0.105400 \\
\hline $\mathrm{Si}$ & 2.136800 & 3.788800 & -0.935100 \\
\hline Si & 3.574900 & 2.279100 & -3.317300 \\
\hline $\mathrm{Si}$ & -0.412400 & -1.038700 & 3.477800 \\
\hline Si & -1.831300 & -3.381500 & 1.893900 \\
\hline K & -4.629800 & -1.087300 & -0.113300 \\
\hline C & -1.398800 & 0.566600 & -4.321100 \\
\hline C & -2.552900 & 0.164300 & -3.707400 \\
\hline C & -0.116800 & 0.749400 & -3.605100 \\
\hline C & -2.625000 & -0.074200 & -2.246200 \\
\hline $\mathrm{H}$ & -3.249500 & 0.686800 & -1.721900 \\
\hline C & 0.545200 & -0.190500 & -2.865200 \\
\hline C & -1.857800 & -0.924700 & -1.489900 \\
\hline $\mathrm{H}$ & -3.494200 & 0.212300 & -4.288900 \\
\hline $\mathrm{H}$ & -1.469700 & 0.918400 & -5.369000 \\
\hline $\mathrm{H}$ & 0.263900 & 1.791200 & -3.624300 \\
\hline C & 0.060600 & -1.596200 & -2.969800 \\
\hline C & -1.111000 & -1.971700 & -2.263600 \\
\hline C & -1.579500 & -3.300300 & -2.381100 \\
\hline $\mathrm{H}$ & -2.501900 & -3.595900 & -1.854400 \\
\hline C & 0.722700 & -2.550900 & -3.775900 \\
\hline
\end{tabular}




$\begin{array}{lrrr}\text { C } & 0.240300 & -3.866000 & -3.889000 \\ \text { C } & -0.915700 & -4.244300 & -3.184400 \\ \text { H } & -1.308500 & -5.270800 & -3.265800 \\ \text { H } & 1.620000 & -2.240100 & -4.335700 \\ \text { H } & 0.762500 & -4.591800 & -4.533100 \\ \text { K } & 2.535200 & 0.742700 & 1.941000 \\ \text { H } & 2.874600 & 1.709200 & 6.147500 \\ \text { C } & 3.636300 & 1.682100 & 5.352200 \\ \text { C } & 4.374800 & 0.504600 & 5.123800 \\ \text { C } & 3.881000 & 2.829200 & 4.571400 \\ \text { C } & 5.357200 & 0.473500 & 4.114100 \\ \text { H } & 4.191500 & -0.389200 & 5.741000 \\ \text { C } & 4.863100 & 2.798200 & 3.562300 \\ \text { H } & 3.308300 & 3.752300 & 4.753800 \\ \text { H } & 5.937900 & -0.446000 & 3.938900 \\ \text { C } & 5.600300 & 1.619800 & 3.332100 \\ \text { H } & 5.057500 & 3.695000 & 2.953400 \\ \text { H } & 6.367600 & 1.597500 & 2.542700 \\ \text { C } & -7.692900 & -2.661100 & 0.195200 \\ \text { C } & -8.033700 & -1.329000 & 0.503700 \\ \text { C } & -7.998400 & -0.341800 & -0.501600 \\ \text { C } & -7.620700 & -0.686600 & -1.814700 \\ \text { C } & -7.278800 & -2.018600 & -2.123000 \\ \text { C } & -7.315700 & -3.005900 & -1.118100 \\ \text { H } & -7.729300 & -3.434800 & 0.978400 \\ \text { H } & -8.337600 & -1.061300 & 1.528300 \\ \text { H } & -8.275000 & 0.697400 & -0.263100 \\ \text { H } & -7.600800 & 0.083100 & -2.602400 \\ \text { H } & -6.990000 & -2.289700 & -3.150900 \\ \text { H } & -7.055900 & -4.048400 & -1.360600\end{array}$

Table S22. Cartesian coordinates of int-1*

Zero-point correction= 1.229878 (Hartree/Particle)

Thermal correction to Energy=

1.311029

Thermal correction to Enthalpy=

Thermal correction to Gibbs Free Energy=

1.104520

Sum of electronic and zero-point Energies $=-5209.555250$

Sum of electronic and thermal Energies $=\quad-5209.474099$

Sum of electronic and thermal Enthalpies $=-5209.473155$

Sum of electronic and thermal Free Energies $=-5209.680608$

$\begin{array}{lrrr}\text { Al } & -0.778900 & -0.156000 & -0.953400 \\ \text { Al } & -0.044300 & -0.518100 & 1.605200 \\ \text { C } & 0.259300 & -4.262300 & -2.721600 \\ \text { H } & 1.021300 & -3.989600 & -1.966500 \\ \text { H } & -0.003200 & -5.330700 & -2.565300 \\ \text { H } & 0.728800 & -4.182100 & -3.724100 \\ \text { C } & -2.210200 & -3.772500 & -0.989100 \\ \text { H } & -3.315600 & -3.665600 & -1.074700 \\ \text { H } & -2.024900 & -4.853900 & -0.815000 \\ \text { H } & -1.842800 & -3.197900 & -0.112000 \\ \text { C } & -2.444900 & -3.853000 & -4.006300 \\ \text { H } & -2.046500 & -3.605000 & -5.011300 \\ \text { H } & -2.524700 & -4.959900 & -3.939600 \\ \text { H } & -3.473300 & -3.439500 & -3.942500 \\ \text { C } & 2.051900 & -1.306500 & -3.461100 \\ \text { H } & 2.240400 & -2.397900 & -3.485600 \\ \text { H } & 2.788800 & -0.820700 & -4.136700 \\ \text { H } & 2.250900 & -0.962100 & -2.423700 \\ \text { C } & 0.302100 & 1.039400 & -4.285900 \\ \text { H } & 0.592700 & 1.597300 & -3.370400\end{array}$




\begin{tabular}{|c|c|c|c|}
\hline $\mathrm{H}$ & 1.028100 & 1.287800 & -5.090000 \\
\hline $\mathrm{H}$ & -0.696400 & 1.407400 & -4.597000 \\
\hline C & -0.006300 & -1.618000 & -5.712700 \\
\hline $\mathrm{H}$ & -1.015100 & -1.371400 & -6.105400 \\
\hline $\mathrm{H}$ & 0.738700 & -1.222100 & -6.436600 \\
\hline $\mathrm{H}$ & 0.092800 & -2.723400 & -5.705100 \\
\hline C & -1.065300 & -1.322000 & -2.706400 \\
\hline C & -2.364300 & -0.707700 & -3.215500 \\
\hline $\mathrm{H}$ & -2.903600 & -1.120000 & -4.088100 \\
\hline C & -2.834800 & 0.461700 & -2.671700 \\
\hline $\mathrm{H}$ & -3.722800 & 0.950900 & -3.121100 \\
\hline C & -2.564400 & 2.474200 & -1.329500 \\
\hline C & -1.720300 & 3.037200 & -0.164900 \\
\hline $\mathrm{H}$ & -0.643200 & 2.913100 & -0.417600 \\
\hline $\mathrm{H}$ & -1.891200 & 2.415900 & 0.741400 \\
\hline C & -2.282600 & 3.363500 & -2.581100 \\
\hline $\mathrm{H}$ & -2.868800 & 2.988800 & -3.447700 \\
\hline $\mathrm{H}$ & -1.211300 & 3.251800 & -2.853600 \\
\hline C & -4.067600 & 2.653500 & -0.941900 \\
\hline $\mathrm{H}$ & -4.271800 & 2.057200 & -0.022200 \\
\hline $\mathrm{H}$ & -4.718000 & 2.252100 & -1.750600 \\
\hline C & -2.053400 & 4.520800 & 0.114800 \\
\hline $\mathrm{H}$ & -1.427200 & 4.879800 & 0.960600 \\
\hline C & -1.761900 & 5.365400 & -1.145600 \\
\hline $\mathrm{H}$ & -0.682800 & 5.300000 & -1.410800 \\
\hline $\mathrm{H}$ & -1.980100 & 6.438800 & -0.948200 \\
\hline C & -2.624600 & 4.845800 & -2.316500 \\
\hline $\mathrm{H}$ & -2.412900 & 5.442100 & -3.232000 \\
\hline C & -4.121500 & 4.968800 & -1.953000 \\
\hline $\mathrm{H}$ & -4.391600 & 6.034700 & -1.781200 \\
\hline $\mathrm{H}$ & -4.750100 & 4.611000 & -2.799100 \\
\hline C & -4.412800 & 4.138400 & -0.682600 \\
\hline $\mathrm{H}$ & -5.490600 & 4.226300 & -0.418300 \\
\hline C & -3.547500 & 4.661700 & 0.484000 \\
\hline $\mathrm{H}$ & -3.791100 & 5.726400 & 0.697600 \\
\hline $\mathrm{H}$ & -3.770400 & 4.091300 & 1.412300 \\
\hline C & -2.308600 & -2.252700 & 3.697100 \\
\hline $\mathrm{H}$ & -1.456600 & -2.771300 & 4.183500 \\
\hline $\mathrm{H}$ & -3.249400 & -2.659000 & 4.127900 \\
\hline $\mathrm{H}$ & -2.275600 & -2.496000 & 2.614500 \\
\hline C & -3.594300 & 0.453600 & 2.972100 \\
\hline $\mathrm{H}$ & -3.227100 & 0.699300 & 1.954100 \\
\hline $\mathrm{H}$ & -4.470500 & -0.228300 & 2.894600 \\
\hline $\mathrm{H}$ & -3.950800 & 1.394900 & 3.436900 \\
\hline C & -2.580700 & -0.072500 & 5.822400 \\
\hline $\mathrm{H}$ & -2.616200 & 1.008700 & 6.071900 \\
\hline $\mathrm{H}$ & -3.569000 & -0.508000 & 6.086200 \\
\hline $\mathrm{H}$ & -1.821200 & -0.547300 & 6.477800 \\
\hline C & 1.264700 & 2.785000 & 2.750900 \\
\hline $\mathrm{H}$ & 0.895700 & 2.896400 & 1.708500 \\
\hline $\mathrm{H}$ & 1.612200 & 3.780300 & 3.102700 \\
\hline $\mathrm{H}$ & 2.134900 & 2.092600 & 2.766800 \\
\hline C & -1.629600 & 3.244500 & 3.693100 \\
\hline $\mathrm{H}$ & -2.371800 & 3.055300 & 4.496400 \\
\hline $\mathrm{H}$ & -1.307200 & 4.304000 & 3.784700 \\
\hline $\mathrm{H}$ & -2.147700 & 3.130000 & 2.721500 \\
\hline C & 0.485000 & 2.349400 & 5.660600 \\
\hline $\mathrm{H}$ & 1.478200 & 1.886700 & 5.833500 \\
\hline $\mathrm{H}$ & 0.573500 & 3.436800 & 5.872900 \\
\hline $\mathrm{H}$ & -0.220500 & 1.919500 & 6.402100 \\
\hline C & -0.456400 & 0.253100 & 3.488600 \\
\hline C & 0.552400 & -0.527300 & 4.316100 \\
\hline $\mathrm{H}$ & 0.644300 & -0.390200 & 5.407000 \\
\hline C & 1.384100 & -1.421600 & 3.702700 \\
\hline $\mathrm{H}$ & 2.125700 & -1.980000 & 4.302200 \\
\hline C & 2.351700 & -2.570900 & 1.790600 \\
\hline C & 3.794200 & -2.024900 & 2.032000 \\
\hline $\mathrm{H}$ & 3.962500 & -1.870300 & 3.119500 \\
\hline
\end{tabular}




\begin{tabular}{|c|c|c|c|}
\hline $\mathrm{H}$ & 3.877200 & -1.018300 & 1.558500 \\
\hline C & 2.255400 & -3.989000 & 2.438600 \\
\hline $\mathrm{H}$ & 1.234000 & -4.389300 & 2.257000 \\
\hline H & 2.376400 & -3.911900 & 3.540200 \\
\hline C & 2.168500 & -2.750900 & 0.268200 \\
\hline $\mathrm{H}$ & 1.144000 & -3.134200 & 0.069400 \\
\hline $\mathrm{H}$ & 2.216300 & -1.755200 & -0.228000 \\
\hline C & 4.865200 & -2.977600 & 1.458300 \\
\hline $\mathrm{H}$ & 5.877000 & -2.558400 & 1.654800 \\
\hline C & 4.734300 & -4.360600 & 2.136200 \\
\hline $\mathrm{H}$ & 5.516800 & -5.050900 & 1.749200 \\
\hline $\mathrm{H}$ & 4.903500 & -4.266200 & 3.232200 \\
\hline C & 3.327700 & -4.941700 & 1.863000 \\
\hline $\mathrm{H}$ & 3.234500 & -5.936700 & 2.352500 \\
\hline C & 3.118900 & -5.088700 & 0.340200 \\
\hline $\mathrm{H}$ & 3.873600 & -5.784800 & -0.089600 \\
\hline $\mathrm{H}$ & 2.119000 & -5.528200 & 0.130200 \\
\hline C & 3.237400 & -3.698700 & -0.322400 \\
\hline $\mathrm{H}$ & 3.074100 & -3.792600 & -1.417900 \\
\hline C & 4.648000 & -3.126100 & -0.062700 \\
\hline $\mathrm{H}$ & 5.420900 & -3.796100 & -0.501100 \\
\hline $\mathrm{H}$ & 4.763600 & -2.139200 & -0.564900 \\
\hline $\mathrm{N}$ & -2.221000 & 1.059500 & -1.573000 \\
\hline $\mathrm{N}$ & 1.330400 & -1.655100 & 2.330100 \\
\hline 0 & -1.383100 & -1.014600 & 0.547700 \\
\hline $\mathrm{Si}$ & -1.323800 & -3.209200 & -2.592800 \\
\hline Si & 0.274400 & -0.844100 & -3.987100 \\
\hline Si & -2.185500 & -0.373700 & 3.979000 \\
\hline $\mathrm{Si}$ & -0.124100 & 2.088700 & 3.870200 \\
\hline K & 2.563200 & 1.402400 & -0.282800 \\
\hline C & 3.783800 & 4.574200 & -0.258700 \\
\hline C & 3.537000 & 4.327500 & -1.623300 \\
\hline C & 4.795800 & 3.868100 & 0.464100 \\
\hline C & 4.308500 & 3.380800 & -2.373000 \\
\hline $\mathrm{H}$ & 4.113800 & 3.223900 & -3.445500 \\
\hline C & 5.527000 & 2.933100 & -0.259900 \\
\hline C & 5.302300 & 2.709100 & -1.672600 \\
\hline $\mathrm{H}$ & 2.746800 & 4.898200 & -2.136400 \\
\hline $\mathrm{H}$ & 3.176200 & 5.327000 & 0.267700 \\
\hline $\mathrm{H}$ & 4.968900 & 4.073000 & 1.532100 \\
\hline C & 6.629800 & 1.908700 & -0.277600 \\
\hline C & 6.408300 & 1.690300 & -1.684200 \\
\hline C & 7.159600 & 0.781200 & -2.417300 \\
\hline $\mathrm{H}$ & 7.007600 & 0.600100 & -3.492600 \\
\hline C & 7.610400 & 1.226700 & 0.430400 \\
\hline C & 8.382600 & 0.292100 & -0.319300 \\
\hline C & 8.164600 & 0.076900 & -1.692400 \\
\hline $\mathrm{H}$ & 8.790400 & -0.658100 & -2.223000 \\
\hline $\mathrm{H}$ & 7.798800 & 1.382000 & 1.504100 \\
\hline $\mathrm{H}$ & 9.174000 & -0.279200 & 0.191300 \\
\hline K & -3.893600 & -1.072000 & -0.182600 \\
\hline $\mathrm{H}$ & -6.968500 & -2.297900 & 2.259900 \\
\hline C & -6.957400 & -2.172200 & 1.165600 \\
\hline C & -7.320600 & -0.936300 & 0.594300 \\
\hline C & -6.589300 & -3.250400 & 0.336600 \\
\hline C & -7.314300 & -0.778700 & -0.805900 \\
\hline $\mathrm{H}$ & -7.615700 & -0.095800 & 1.242300 \\
\hline C & -6.583500 & -3.092700 & -1.063600 \\
\hline $\mathrm{H}$ & -6.310200 & -4.218100 & 0.782900 \\
\hline C & -6.945500 & -1.856700 & -1.635000 \\
\hline $\mathrm{H}$ & -7.604800 & 0.185300 & -1.252900 \\
\hline $\mathrm{H}$ & -6.300800 & -3.936900 & -1.712100 \\
\hline $\mathrm{H}$ & -6.943100 & -1.735000 & -2.729700 \\
\hline
\end{tabular}

Table S23. Cartesian coordinates of TS-1* 
Zero-point correction= 1.229093 (Hartree/Particle)

Thermal correction to Energy=

1.308428

Thermal correction to Enthalpy=

1.309372

Thermal correction to Gibbs Free Energy=

1.113502

Sum of electronic and zero-point Energies $=-5209.509019$

Sum of electronic and thermal Energies= $\quad-5209.429685$

Sum of electronic and thermal Enthalpies $=-5209.428740$

Sum of electronic and thermal Free Energies= -5209.624610

\begin{tabular}{|c|c|c|c|}
\hline$A l$ & -0.451000 & -1.283400 & 0.462200 \\
\hline Al & 1.013400 & 1.824100 & -0.163000 \\
\hline C & 1.444600 & -0.224000 & 4.483400 \\
\hline $\mathrm{H}$ & 2.191900 & -0.340200 & 3.674200 \\
\hline $\mathrm{H}$ & 1.718100 & 0.673900 & 5.077500 \\
\hline $\mathrm{H}$ & 1.537000 & -1.104400 & 5.150800 \\
\hline $\mathrm{C}$ & -0.455500 & 1.791400 & 3.231500 \\
\hline $\mathrm{H}$ & -1.499200 & 2.178700 & 3.232900 \\
\hline $\mathrm{H}$ & 0.093500 & 2.425800 & 3.960400 \\
\hline $\mathrm{H}$ & 0.003200 & 1.976700 & 2.230200 \\
\hline$C$ & -1.463500 & 0.021600 & 5.366000 \\
\hline $\mathrm{H}$ & -1.462500 & -0.918500 & 5.949900 \\
\hline $\mathrm{H}$ & -1.116200 & 0.836800 & 6.037800 \\
\hline $\mathrm{H}$ & -2.515500 & 0.244600 & 5.087800 \\
\hline $\mathrm{C}$ & 1.468700 & -3.476600 & 3.091600 \\
\hline $\mathrm{H}$ & 2.069500 & -2.858400 & 3.788700 \\
\hline $\mathrm{H}$ & 1.680100 & -4.542900 & 3.317600 \\
\hline $\mathrm{H}$ & 1.824900 & -3.288200 & 2.059300 \\
\hline $\mathrm{C}$ & -1.431700 & -4.461300 & 2.425700 \\
\hline $\mathrm{H}$ & -1.379200 & -4.404400 & 1.322400 \\
\hline $\mathrm{H}$ & -1.069700 & -5.466600 & 2.728700 \\
\hline $\mathrm{H}$ & -2.493800 & -4.368300 & 2.731700 \\
\hline $\mathrm{C}$ & -0.795600 & -3.370600 & 5.128700 \\
\hline $\mathrm{H}$ & -1.858500 & -3.146600 & 5.357900 \\
\hline $\mathrm{H}$ & -0.627000 & -4.442600 & 5.371300 \\
\hline $\mathrm{H}$ & -0.159000 & -2.776700 & 5.816200 \\
\hline $\mathrm{C}$ & -0.870700 & -1.381600 & 2.572800 \\
\hline $\mathrm{C}$ & -2.392600 & -1.421000 & 2.542000 \\
\hline $\mathrm{H}$ & -2.987100 & -1.503100 & 3.471400 \\
\hline$C$ & -3.057500 & -1.554500 & 1.350100 \\
\hline $\mathrm{H}$ & -4.152900 & -1.716900 & 1.350900 \\
\hline $\mathrm{C}$ & -3.178300 & -1.851700 & -1.068900 \\
\hline $\mathrm{C}$ & -2.299000 & -1.711600 & -2.329800 \\
\hline $\mathrm{H}$ & -1.398200 & -2.345200 & -2.197500 \\
\hline $\mathrm{H}$ & -1.934700 & -0.662200 & -2.408600 \\
\hline$C$ & -3.680800 & -3.326000 & -0.994600 \\
\hline $\mathrm{H}$ & -4.324100 & -3.456200 & -0.097300 \\
\hline $\mathrm{H}$ & -2.801400 & -3.988300 & -0.847700 \\
\hline$C$ & -4.429600 & -0.936600 & -1.275300 \\
\hline $\mathrm{H}$ & -4.094500 & 0.123500 & -1.369800 \\
\hline $\mathrm{H}$ & -5.100400 & -0.992400 & -0.389900 \\
\hline $\mathrm{C}$ & -3.068900 & -2.108800 & -3.609100 \\
\hline $\mathrm{H}$ & -2.397700 & -1.997700 & -4.490000 \\
\hline C & -3.537200 & -3.576800 & -3.498200 \\
\hline $\mathrm{H}$ & -2.658200 & -4.250900 & -3.401900 \\
\hline $\mathrm{H}$ & -4.075200 & -3.879900 & -4.424100 \\
\hline $\mathrm{C}$ & -4.458100 & -3.726200 & -2.267100 \\
\hline $\mathrm{H}$ & -4.793900 & -4.782800 & -2.176700 \\
\hline $\mathrm{C}$ & -5.687400 & -2.802800 & -2.425800 \\
\hline $\mathrm{H}$ & -6.273700 & -3.088200 & -3.327500 \\
\hline $\mathrm{H}$ & -6.367700 & -2.916600 & -1.552200 \\
\hline $\mathrm{C}$ & -5.215900 & -1.336100 & -2.546500 \\
\hline $\mathrm{H}$ & -6.099400 & -0.667600 & -2.655000 \\
\hline$C$ & -4.298700 & -1.188200 & -3.779900 \\
\hline $\mathrm{H}$ & -4.855100 & -1.452800 & -4.706600 \\
\hline $\mathrm{H}$ & -3.974300 & -0.129100 & -3.891000 \\
\hline$C$ & 1.732500 & 4.834400 & 1.368200 \\
\hline
\end{tabular}




\begin{tabular}{|c|c|c|c|}
\hline $\mathrm{H}$ & 2.773900 & 4.591000 & 1.074100 \\
\hline $\mathrm{H}$ & 1.754600 & 5.798800 & 1.920900 \\
\hline $\mathrm{H}$ & 1.382300 & 4.052600 & 2.073900 \\
\hline C & -1.170200 & 5.246600 & 0.479600 \\
\hline $\mathrm{H}$ & -1.891700 & 5.483500 & -0.329500 \\
\hline $\mathrm{H}$ & -1.536300 & 4.352400 & 1.027400 \\
\hline $\mathrm{H}$ & -1.186400 & 6.094400 & 1.197700 \\
\hline C & 1.135100 & 6.634400 & -0.993300 \\
\hline $\mathrm{H}$ & 0.535800 & 6.881000 & -1.891400 \\
\hline $\mathrm{H}$ & 1.015300 & 7.467200 & -0.267500 \\
\hline $\mathrm{H}$ & 2.203400 & 6.606600 & -1.291100 \\
\hline C & -0.032900 & 2.178800 & -4.097800 \\
\hline $\mathrm{H}$ & -0.170300 & 1.122200 & -3.782300 \\
\hline $\mathrm{H}$ & -0.707300 & 2.325600 & -4.968000 \\
\hline $\mathrm{H}$ & 1.001000 & 2.338300 & -4.473700 \\
\hline C & -2.216100 & 2.852200 & -2.109600 \\
\hline $\mathrm{H}$ & -2.579500 & 3.443900 & -1.244200 \\
\hline $\mathrm{H}$ & -2.967600 & 2.957600 & -2.920700 \\
\hline $\mathrm{H}$ & -2.169700 & 1.775500 & -1.842000 \\
\hline C & -0.743800 & 5.084300 & -3.622500 \\
\hline $\mathrm{H}$ & 0.215700 & 5.487800 & -4.008100 \\
\hline $\mathrm{H}$ & -1.422200 & 4.947200 & -4.491800 \\
\hline H & -1.195100 & 5.857800 & -2.967800 \\
\hline C & 0.799100 & 3.485500 & -1.337800 \\
\hline C & 2.226000 & 3.611900 & -1.854200 \\
\hline $\mathrm{H}$ & 2.524300 & 4.420000 & -2.545500 \\
\hline C & 3.204300 & 2.792300 & -1.348200 \\
\hline $\mathrm{H}$ & 4.263000 & 2.947300 & -1.625800 \\
\hline C & 3.999600 & 1.063600 & 0.239600 \\
\hline C & 5.088600 & 0.513200 & -0.731300 \\
\hline $\mathrm{H}$ & 5.522600 & 1.337000 & -1.339400 \\
\hline $\mathrm{H}$ & 4.633100 & -0.220500 & -1.434500 \\
\hline C & 4.689000 & 2.039100 & 1.238300 \\
\hline $\mathrm{H}$ & 3.917100 & 2.430500 & 1.936600 \\
\hline $\mathrm{H}$ & 5.091600 & 2.915500 & 0.682800 \\
\hline C & 3.439000 & -0.133800 & 1.040200 \\
\hline $\mathrm{H}$ & 2.648500 & 0.234700 & 1.731700 \\
\hline $\mathrm{H}$ & 2.950700 & -0.862800 & 0.354000 \\
\hline C & 6.218600 & -0.195000 & 0.053800 \\
\hline $\mathrm{H}$ & 6.976000 & -0.571700 & -0.668700 \\
\hline C & 6.878900 & 0.808200 & 1.025500 \\
\hline $\mathrm{H}$ & 7.709900 & 0.314000 & 1.575500 \\
\hline $\mathrm{H}$ & 7.327700 & 1.654600 & 0.458600 \\
\hline C & 5.819900 & 1.333900 & 2.019700 \\
\hline $\mathrm{H}$ & 6.289800 & 2.060700 & 2.718700 \\
\hline C & 5.223400 & 0.151600 & 2.815300 \\
\hline $\mathrm{H}$ & 6.020200 & -0.361800 & 3.397800 \\
\hline $\mathrm{H}$ & 4.478100 & 0.522900 & 3.552600 \\
\hline C & 4.560100 & -0.841700 & 1.835700 \\
\hline $\mathrm{H}$ & 4.114700 & -1.690200 & 2.397400 \\
\hline C & 5.619500 & -1.376700 & 0.847800 \\
\hline $\mathrm{H}$ & 6.421800 & -1.915300 & 1.398600 \\
\hline $\mathrm{H}$ & 5.150700 & -2.108700 & 0.155400 \\
\hline N & -2.392300 & -1.480800 & 0.126000 \\
\hline $\mathrm{N}$ & 2.889300 & 1.740700 & -0.477500 \\
\hline 0 & 0.002400 & 0.424400 & -0.025100 \\
\hline $\mathrm{Si}$ & -0.331000 & -0.039100 & 3.822200 \\
\hline $\mathrm{Si}$ & -0.385200 & -3.121600 & 3.273500 \\
\hline $\mathrm{Si}$ & 0.612300 & 5.003700 & -0.160900 \\
\hline $\mathrm{Si}$ & -0.501900 & 3.422100 & -2.723300 \\
\hline K & 2.274500 & 0.237200 & -2.861300 \\
\hline C & 3.222200 & -2.136900 & -4.471200 \\
\hline C & 1.830700 & -2.060500 & -4.738400 \\
\hline C & 3.720100 & -2.510100 & -3.192800 \\
\hline C & 0.861200 & -2.348300 & -3.719900 \\
\hline $\mathrm{H}$ & -0.207400 & -2.359700 & -3.984100 \\
\hline C & 2.765600 & -2.805700 & -2.194400 \\
\hline C & 1.331800 & -2.674000 & -2.448200 \\
\hline
\end{tabular}




$\begin{array}{lrrr}\text { H } & 1.487300 & -1.830100 & -5.759900 \\ \mathrm{H} & 3.935500 & -1.947300 & -5.291300 \\ \mathrm{H} & 4.802100 & -2.657500 & -3.040400 \\ \mathrm{C} & 2.508200 & -3.484400 & -0.933900 \\ \mathrm{C} & 1.031100 & -3.334100 & -1.110700 \\ \mathrm{C} & 0.255500 & -4.496700 & -0.753800 \\ \mathrm{H} & -0.789800 & -4.614800 & -1.087100 \\ \mathrm{C} & 3.086600 & -4.488300 & -0.134300 \\ \mathrm{C} & 2.228400 & -5.460400 & 0.404900 \\ \mathrm{C} & 0.843000 & -5.484200 & 0.037300 \\ \mathrm{H} & 0.226500 & -6.335700 & 0.369700 \\ \mathrm{H} & 4.180700 & -4.588000 & -0.026200 \\ \mathrm{H} & 2.632400 & -6.267000 & 1.036900 \\ \mathrm{~K} & -2.607100 & 1.337000 & 0.903500 \\ \mathrm{H} & -4.212700 & 2.458800 & 4.414900 \\ \mathrm{C} & -4.643100 & 2.591800 & 3.409800 \\ \mathrm{C} & -4.391300 & 3.773300 & 2.685800 \\ \mathrm{C} & -5.453300 & 1.580600 & 2.852900 \\ \mathrm{C} & -4.949800 & 3.948200 & 1.402500 \\ \mathrm{H} & -3.766400 & 4.566200 & 3.126500 \\ \mathrm{C} & -6.011600 & 1.755800 & 1.570000 \\ \mathrm{H} & -5.649800 & 0.658000 & 3.420400 \\ \mathrm{H} & -4.760800 & 4.877100 & 0.841800 \\ \mathrm{C} & -5.759800 & 2.936900 & 0.845400 \\ \mathrm{H} & -6.652500 & 0.971400 & 1.137500 \\ \mathrm{H} & -6.206100 & 3.075800 & -0.152300\end{array}$

Table S24. Cartesian coordinates of int-2 ${ }^{*}$

Zero-point correction=

Thermal correction to Energy=

Thermal correction to Enthalpy=

Thermal correction to Gibbs Free Energy=

Sum of electronic and zero-point Energies $=\quad-5209.525140$

Sum of electronic and thermal Energies $=\quad-5209.445848$

Sum of electronic and thermal Enthalpies $=\quad-5209.444904$

Sum of electronic and thermal Free Energies $=-5209.639523$

1.309408

1.310352

1.115734

$-5209.445848$
1.230116 (Hartree/Particle)

Al $\quad 0.786500$

Al -1.427800

$-1.345600$

$-1.644400$

$-2.168900$

$-1.236600$

$-0.136500$

0.739200

$-0.944500$

$-0.479400$

0.950300

1.047000

0.234500

1.938000

0.887100

0.524100

1. 354400

0.017000

3.261800

2. 711200

3.630600

4.145200

3.429900

4.352000

3.736300
$-0.076500$
0.069200
$-3.353500$
$-2.303300$
$-3.869300$
$-3.845400$
$-3.530900$
$-3.871100$
$-4.271100$
$-2.540300$
$-5.229900$
$-5.474400$
$-5.958200$
$-5.402800$
$-2.767100$
$-3.809300$
$-2.697200$
$-2.081000$
$-0.883000$
0.073400
$-1.119000$
$-0.761500$
$-3.782800$
$-3.566500$
$-3.919300$ 


\begin{tabular}{|c|c|c|c|}
\hline $\mathrm{H}$ & 3.023100 & -3.403600 & -4.751200 \\
\hline C & 1.457500 & -1.922600 & -2.029400 \\
\hline C & 2.776000 & -1.158700 & -2.048400 \\
\hline $\mathrm{H}$ & 3.382800 & -1.062500 & -2.968000 \\
\hline C & 3.354800 & -0.784800 & -0.858300 \\
\hline $\mathrm{H}$ & 4.395900 & -0.404100 & -0.853100 \\
\hline C & 3.463200 & -0.717000 & 1.577000 \\
\hline C & 2.524100 & -0.657700 & 2.800200 \\
\hline $\mathrm{H}$ & 1.889900 & -1.566700 & 2.796800 \\
\hline $\mathrm{H}$ & 1.836400 & 0.211000 & 2.695100 \\
\hline C & 4.404800 & -1.945900 & 1.764100 \\
\hline $\mathrm{H}$ & 5.096100 & -2.014000 & 0.895400 \\
\hline $\mathrm{H}$ & 3.788400 & -2.868900 & 1.748600 \\
\hline C & 4.356600 & 0.563900 & 1.607000 \\
\hline $\mathrm{H}$ & 3.710700 & 1.462500 & 1.485900 \\
\hline $\mathrm{H}$ & 5.070300 & 0.556900 & 0.754600 \\
\hline C & 3.313300 & -0.562000 & 4.125100 \\
\hline $\mathrm{H}$ & 2.595800 & -0.526800 & 4.975100 \\
\hline C & 4.227800 & -1.798400 & 4.273300 \\
\hline $\mathrm{H}$ & 3.614100 & -2.725100 & 4.305200 \\
\hline $\mathrm{H}$ & 4.787900 & -1.749000 & 5.234000 \\
\hline C & 5.209300 & -1.851200 & 3.080800 \\
\hline $\mathrm{H}$ & 5.865900 & -2.744300 & 3.175100 \\
\hline C & 6.072400 & -0.570300 & 3.069100 \\
\hline $\mathrm{H}$ & 6.670400 & -0.497100 & 4.004900 \\
\hline $\mathrm{H}$ & 6.799100 & -0.604800 & 2.226600 \\
\hline C & 5.155300 & 0.665400 & 2.927900 \\
\hline $\mathrm{H}$ & 5.775200 & 1.590200 & 2.910600 \\
\hline C & 4.176000 & 0.718800 & 4.121400 \\
\hline $\mathrm{H}$ & 4.739600 & 0.809600 & 5.076800 \\
\hline $\mathrm{H}$ & 3.526700 & 1.620000 & 4.045100 \\
\hline C & -2.195300 & 3.701200 & -2.321900 \\
\hline $\mathrm{H}$ & -3.223900 & 3.345300 & -2.109600 \\
\hline $\mathrm{H}$ & -2.264100 & 4.454900 & -3.135800 \\
\hline $\mathrm{H}$ & -1.613100 & 2.841600 & -2.718500 \\
\hline C & 0.318200 & 5.098700 & -1.277800 \\
\hline $\mathrm{H}$ & 0.834100 & 5.649200 & -0.464700 \\
\hline $\mathrm{H}$ & 1.001400 & 4.302500 & -1.635900 \\
\hline $\mathrm{H}$ & 0.195700 & 5.805200 & -2.127000 \\
\hline C & -2.421100 & 6.058800 & -0.400200 \\
\hline $\mathrm{H}$ & -2.093600 & 6.566400 & 0.529100 \\
\hline $\mathrm{H}$ & -2.299600 & 6.778100 & -1.238200 \\
\hline $\mathrm{H}$ & -3.502700 & 5.834500 & -0.305300 \\
\hline C & -0.604100 & 2.648300 & 3.675700 \\
\hline $\mathrm{H}$ & -0.449100 & 1.560200 & 3.502900 \\
\hline $\mathrm{H}$ & 0.096400 & 2.937400 & 4.487800 \\
\hline $\mathrm{H}$ & -1.627600 & 2.835400 & 4.068100 \\
\hline C & 1.547400 & 3.339100 & 1.630600 \\
\hline $\mathrm{H}$ & 1.874300 & 3.930800 & 0.750800 \\
\hline $\mathrm{H}$ & 2.234900 & 3.588300 & 2.465900 \\
\hline $\mathrm{H}$ & 1.666400 & 2.254600 & 1.424400 \\
\hline C & -0.386300 & 5.511300 & 2.670900 \\
\hline $\mathrm{H}$ & -1.420600 & 5.773900 & 2.977600 \\
\hline $\mathrm{H}$ & 0.273800 & 5.689700 & 3.546600 \\
\hline $\mathrm{H}$ & -0.080500 & 6.221900 & 1.875400 \\
\hline C & -1.455200 & 3.249200 & 0.714700 \\
\hline C & -2.911600 & 3.326600 & 1.153200 \\
\hline $\mathrm{H}$ & -3.318400 & 4.223200 & 1.651700 \\
\hline C & -3.767800 & 2.311700 & 0.815100 \\
\hline $\mathrm{H}$ & -4.850000 & 2.391500 & 1.026600 \\
\hline C & -4.307900 & 0.249700 & -0.431200 \\
\hline C & -5.310700 & -0.331000 & 0.608800 \\
\hline $\mathrm{H}$ & -5.840100 & 0.492700 & 1.141700 \\
\hline $\mathrm{H}$ & -4.752200 & -0.932500 & 1.361800 \\
\hline C & -5.113300 & 1.030100 & -1.509700 \\
\hline $\mathrm{H}$ & -4.398800 & 1.438800 & -2.257400 \\
\hline $\mathrm{H}$ & -5.630500 & 1.901100 & -1.049500 \\
\hline C & -3.596500 & -0.928600 & -1.124700 \\
\hline
\end{tabular}




\begin{tabular}{|c|c|c|c|}
\hline $\mathrm{H}$ & -2.891200 & -0.523100 & -1.884300 \\
\hline $\mathrm{H}$ & -2.990100 & -1.504400 & -0.384600 \\
\hline C & -6.352400 & -1.243000 & -0.083700 \\
\hline $\mathrm{H}$ & -7.056100 & -1.634900 & 0.683400 \\
\hline C & -7.131000 & -0.433300 & -1.144000 \\
\hline $\mathrm{H}$ & -7.896000 & -1.078900 & -1.628700 \\
\hline $\mathrm{H}$ & -7.681100 & 0.406400 & -0.662200 \\
\hline C & -6.145700 & 0.112000 & -2.201700 \\
\hline $\mathrm{H}$ & -6.702800 & 0.701500 & -2.963300 \\
\hline C & -5.408600 & -1.062900 & -2.881500 \\
\hline $\mathrm{H}$ & -6.137400 & -1.726100 & -3.398100 \\
\hline $\mathrm{H}$ & -4.714900 & -0.680000 & -3.662700 \\
\hline C & -4.624600 & -1.857200 & -1.813900 \\
\hline $\mathrm{H}$ & -4.077600 & -2.696300 & -2.291400 \\
\hline C & -5.610900 & -2.414600 & -0.764300 \\
\hline $\mathrm{H}$ & -6.342000 & -3.097100 & -1.251500 \\
\hline $\mathrm{H}$ & -5.063300 & -3.014800 & -0.006400 \\
\hline $\mathrm{N}$ & 2.655300 & -0.825400 & 0.343000 \\
\hline $\mathrm{N}$ & -3.296700 & 1.138800 & 0.204600 \\
\hline 0 & -0.110900 & 0.288500 & -0.236500 \\
\hline Si & 0.284700 & -1.491100 & -3.457600 \\
\hline $\mathrm{Si}$ & 2.181300 & -3.709000 & -2.321800 \\
\hline $\mathrm{Si}$ & -1.420000 & 4.485700 & -0.774800 \\
\hline Si & -0.252900 & 3.693400 & 2.120700 \\
\hline K & -3.085300 & 0.507100 & 3.133000 \\
\hline C & -3.002200 & -2.070400 & 4.470700 \\
\hline C & -1.747500 & -1.516100 & 4.829100 \\
\hline C & -3.283700 & -2.532500 & 3.160600 \\
\hline C & -0.669400 & -1.467100 & 3.867100 \\
\hline $\mathrm{H}$ & 0.322300 & -1.124700 & 4.202200 \\
\hline C & -2.197800 & -2.524800 & 2.224300 \\
\hline C & -0.898900 & -1.983100 & 2.596700 \\
\hline $\mathrm{H}$ & -1.556100 & -1.220900 & 5.873500 \\
\hline $\mathrm{H}$ & -3.782600 & -2.164200 & 5.247800 \\
\hline $\mathrm{H}$ & -4.242700 & -3.032600 & 2.945400 \\
\hline C & -1.700300 & -3.080700 & 1.009400 \\
\hline C & -0.240400 & -2.573800 & 1.311800 \\
\hline C & 0.611200 & -3.813300 & 1.501200 \\
\hline $\mathrm{H}$ & 1.520500 & -3.766000 & 2.129100 \\
\hline C & -1.983300 & -4.297300 & 0.318600 \\
\hline C & -0.954800 & -5.216000 & 0.159500 \\
\hline C & 0.293200 & -4.994400 & 0.876100 \\
\hline $\mathrm{H}$ & 0.989700 & -5.846300 & 0.968300 \\
\hline $\mathrm{H}$ & -3.020500 & -4.572300 & 0.048200 \\
\hline $\mathrm{H}$ & -1.129300 & -6.182100 & -0.339500 \\
\hline K & 2.014200 & 1.715300 & -1.387700 \\
\hline $\mathrm{H}$ & 3.990000 & 1.533100 & -4.953700 \\
\hline C & 4.141600 & 2.275800 & -4.154700 \\
\hline C & 5.058600 & 2.013400 & -3.116500 \\
\hline C & 3.425400 & 3.487900 & -4.173600 \\
\hline C & 5.256700 & 2.965600 & -2.096500 \\
\hline $\mathrm{H}$ & 5.621000 & 1.067100 & -3.106500 \\
\hline C & 3.623400 & 4.440900 & -3.153200 \\
\hline $\mathrm{H}$ & 2.718700 & 3.696300 & -4.992800 \\
\hline C & 4.538700 & 4.177300 & -2.113300 \\
\hline $\mathrm{H}$ & 5.979200 & 2.764800 & -1.289600 \\
\hline $\mathrm{H}$ & 3.074600 & 5.395300 & -3.175400 \\
\hline $\mathrm{H}$ & 4.704500 & 4.926400 & -1.322400 \\
\hline
\end{tabular}

Table S25. Cartesian coordinates of TS-2* Zero-point correction= Thermal correction to Energy= 1.230650 (Hartree/Particle) Thermal correction to Enthalpy= 
Sum of electronic and zero-point Energies $=-5209.514572$

Sum of electronic and thermal Energies= $\quad-5209.436116$

Sum of electronic and thermal Enthalpies $=-5209.435172$

Sum of electronic and thermal Free Energies= -5209.627266

\begin{tabular}{|c|c|c|c|}
\hline $\mathrm{Al}$ & -0.907600 & 1.316600 & 0.264200 \\
\hline $\mathrm{Al}$ & 1.522500 & -0.924300 & -0.040800 \\
\hline C & 1.494600 & 3.388600 & -2.362600 \\
\hline $\mathrm{H}$ & 1.797600 & 3.120200 & -1.330800 \\
\hline $\mathrm{H}$ & 2.273000 & 3.030100 & -3.069000 \\
\hline $\mathrm{H}$ & 1.476600 & 4.495400 & -2.420100 \\
\hline C & 0.091600 & 0.918300 & -3.565800 \\
\hline $\mathrm{H}$ & -0.831700 & 0.526900 & -4.049700 \\
\hline $\mathrm{H}$ & 0.864700 & 0.971700 & -4.361500 \\
\hline $\mathrm{H}$ & 0.442500 & 0.217300 & -2.781800 \\
\hline$C$ & -0.807600 & 3.649000 & -4.337200 \\
\hline $\mathrm{H}$ & -0.853200 & 4.740600 & -4.147500 \\
\hline $\mathrm{H}$ & -0.094400 & 3.489600 & -5.175500 \\
\hline $\mathrm{H}$ & -1.810100 & 3.324000 & -4.687500 \\
\hline $\mathrm{C}$ & -0.625900 & 5.713800 & -0.858900 \\
\hline $\mathrm{H}$ & -0.280200 & 5.993900 & -1.876200 \\
\hline $\mathrm{H}$ & -1.011500 & 6.637400 & -0.375900 \\
\hline $\mathrm{H}$ & 0.249600 & 5.362100 & -0.279300 \\
\hline$C$ & -3.055300 & 4.499900 & 0.650300 \\
\hline $\mathrm{H}$ & -2.481300 & 4.228800 & 1.555300 \\
\hline $\mathrm{H}$ & -3.436100 & 5.536700 & 0.775300 \\
\hline $\mathrm{H}$ & -3.931000 & 3.826000 & 0.567500 \\
\hline$C$ & -3.244500 & 5.077600 & -2.250600 \\
\hline $\mathrm{H}$ & -4.201200 & 4.515400 & -2.239700 \\
\hline $\mathrm{H}$ & -3.481000 & 6.132300 & -1.988200 \\
\hline $\mathrm{H}$ & -2.856500 & 5.075400 & -3.287900 \\
\hline$C$ & -1.411200 & 2.611600 & -1.344600 \\
\hline$C$ & -2.743500 & 1.983000 & -1.737200 \\
\hline $\mathrm{H}$ & -3.269700 & 2.268700 & -2.666900 \\
\hline$C$ & -3.412500 & 1.185900 & -0.841200 \\
\hline $\mathrm{H}$ & -4.450800 & 0.864900 & -1.060800 \\
\hline$C$ & -3.721900 & 0.233500 & 1.379200 \\
\hline $\mathrm{C}$ & -2.904200 & -0.221900 & 2.605800 \\
\hline $\mathrm{H}$ & -2.316800 & 0.646400 & 2.967800 \\
\hline $\mathrm{H}$ & -2.165300 & -0.994100 & 2.299100 \\
\hline$C$ & -4.711500 & 1.335600 & 1.872400 \\
\hline $\mathrm{H}$ & -5.320800 & 1.698100 & 1.015300 \\
\hline $\mathrm{H}$ & -4.121900 & 2.205100 & 2.231500 \\
\hline$C$ & -4.580600 & -0.977900 & 0.892900 \\
\hline $\mathrm{H}$ & -3.904100 & -1.789100 & 0.543900 \\
\hline $\mathrm{H}$ & -5.207200 & -0.678200 & 0.024200 \\
\hline$C$ & -3.812500 & -0.761900 & 3.732800 \\
\hline $\mathrm{H}$ & -3.178400 & -1.086300 & 4.588100 \\
\hline$C$ & -4.775000 & 0.354500 & 4.194900 \\
\hline $\mathrm{H}$ & -4.196300 & 1.214400 & 4.597900 \\
\hline $\mathrm{H}$ & -5.424700 & -0.012600 & 5.020800 \\
\hline$C$ & -5.634900 & 0.809500 & 2.994900 \\
\hline $\mathrm{H}$ & -6.322100 & 1.624000 & 3.315100 \\
\hline$C$ & -6.459100 & -0.387300 & 2.470100 \\
\hline $\mathrm{H}$ & -7.143200 & -0.763700 & 3.263200 \\
\hline $\mathrm{H}$ & -7.101300 & -0.067000 & 1.619200 \\
\hline$C$ & -5.499300 & -1.510200 & 2.017600 \\
\hline $\mathrm{H}$ & -6.088300 & -2.373600 & 1.633800 \\
\hline$C$ & -4.633900 & -1.963300 & 3.214000 \\
\hline $\mathrm{H}$ & -5.279500 & -2.368500 & 4.025200 \\
\hline $\mathrm{H}$ & -3.954900 & -2.788800 & 2.903900 \\
\hline$C$ & 2.283100 & -2.510600 & -2.986200 \\
\hline $\mathrm{H}$ & 3.303200 & -2.209400 & -2.679200 \\
\hline $\mathrm{H}$ & 2.357900 & -3.021100 & -3.971100 \\
\hline $\mathrm{H}$ & 1.689500 & -1.584600 & -3.140500 \\
\hline$C$ & -0.150100 & -4.243900 & -2.533500 \\
\hline $\mathrm{H}$ & -0.682500 & -5.031100 & -1.963000 \\
\hline
\end{tabular}




\begin{tabular}{|c|c|c|c|}
\hline $\mathrm{H}$ & -0.867500 & -3.421500 & -2.724600 \\
\hline $\mathrm{H}$ & 0.097600 & -4.670900 & -3.529500 \\
\hline C & 2.573100 & -5.293900 & -1.811600 \\
\hline $\mathrm{H}$ & 2.264100 & -6.064400 & -1.077200 \\
\hline $\mathrm{H}$ & 2.483200 & -5.739600 & -2.825600 \\
\hline $\mathrm{H}$ & 3.643500 & -5.065700 & -1.637500 \\
\hline C & 0.107900 & -3.548700 & 2.971000 \\
\hline $\mathrm{H}$ & 0.023000 & -2.473100 & 3.241200 \\
\hline $\mathrm{H}$ & -0.790500 & -4.040500 & 3.401600 \\
\hline $\mathrm{H}$ & 0.969200 & -4.015700 & 3.498400 \\
\hline C & -1.647200 & -3.434000 & 0.517200 \\
\hline H & -1.840200 & -3.708400 & -0.538900 \\
\hline H & -2.408800 & -3.954500 & 1.134600 \\
\hline $\mathrm{H}$ & -1.798400 & -2.343600 & 0.655300 \\
\hline C & 0.264800 & -5.806900 & 1.002700 \\
\hline $\mathrm{H}$ & 1.264800 & -6.149900 & 1.343500 \\
\hline $\mathrm{H}$ & -0.489600 & -6.279400 & 1.667900 \\
\hline $\mathrm{H}$ & 0.105800 & -6.210600 & -0.017900 \\
\hline C & 1.435800 & -2.999200 & 0.087700 \\
\hline C & 2.864900 & -3.285600 & 0.526400 \\
\hline $\mathrm{H}$ & 3.206500 & -4.289400 & 0.837100 \\
\hline C & 3.798800 & -2.294100 & 0.327700 \\
\hline H & 4.869300 & -2.485500 & 0.527900 \\
\hline C & 4.542800 & -0.108000 & -0.476400 \\
\hline C & 5.336700 & 0.334500 & 0.785900 \\
\hline $\mathrm{H}$ & 5.760400 & -0.561200 & 1.296200 \\
\hline $\mathrm{H}$ & 4.632000 & 0.817400 & 1.496300 \\
\hline C & 5.541000 & -0.774000 & -1.473000 \\
\hline $\mathrm{H}$ & 4.987000 & -1.083800 & -2.385900 \\
\hline $\mathrm{H}$ & 5.976500 & -1.697400 & -1.033400 \\
\hline C & 3.975700 & 1.152800 & -1.161900 \\
\hline $\mathrm{H}$ & 3.422700 & 0.847700 & -2.078600 \\
\hline $\mathrm{H}$ & 3.240200 & 1.631500 & -0.482400 \\
\hline C & 6.483700 & 1.301500 & 0.408900 \\
\hline $\mathrm{H}$ & 7.031600 & 1.596600 & 1.331200 \\
\hline C & 7.450800 & 0.602200 & -0.572100 \\
\hline $\mathrm{H}$ & 8.292900 & 1.282200 & -0.829100 \\
\hline $\mathrm{H}$ & 7.900700 & -0.298500 & -0.096400 \\
\hline C & 6.680900 & 0.199000 & -1.850000 \\
\hline $\mathrm{H}$ & 7.371700 & -0.312400 & -2.556700 \\
\hline C & 6.082700 & 1.454900 & -2.521400 \\
\hline $\mathrm{H}$ & 6.893600 & 2.158500 & -2.814100 \\
\hline $\mathrm{H}$ & 5.550200 & 1.171400 & -3.456300 \\
\hline C & 5.109500 & 2.138400 & -1.535600 \\
\hline $\mathrm{H}$ & 4.658200 & 3.036700 & -2.008600 \\
\hline C & 5.891000 & 2.556000 & -0.269200 \\
\hline $\mathrm{H}$ & 6.704800 & 3.264700 & -0.540900 \\
\hline $\mathrm{H}$ & 5.225000 & 3.095800 & 0.437200 \\
\hline $\mathrm{N}$ & -2.806300 & 0.733600 & 0.330900 \\
\hline $\mathrm{N}$ & 3.432900 & -1.033400 & -0.129200 \\
\hline 0 & 0.063600 & -0.095900 & -0.440400 \\
\hline Si & -0.200900 & 2.660700 & -2.812200 \\
\hline Si & -2.013500 & 4.410100 & -0.936100 \\
\hline Si & 1.510200 & -3.712100 & -1.731800 \\
\hline Si & 0.100200 & -3.906100 & 1.090200 \\
\hline K & 2.752300 & -1.956400 & 3.235500 \\
\hline C & 2.359400 & 0.181900 & 5.203200 \\
\hline C & 1.005300 & -0.193000 & 5.340300 \\
\hline C & 2.850200 & 0.816400 & 4.028700 \\
\hline C & 0.034300 & 0.178000 & 4.340700 \\
\hline $\mathrm{H}$ & -1.033600 & 0.015900 & 4.556000 \\
\hline C & 1.902600 & 1.080800 & 2.998400 \\
\hline C & 0.486900 & 0.837200 & 3.205700 \\
\hline $\mathrm{H}$ & 0.661100 & -0.657400 & 6.278500 \\
\hline $\mathrm{H}$ & 3.049400 & 0.005400 & 6.048700 \\
\hline $\mathrm{H}$ & 3.889100 & 1.184900 & 3.983300 \\
\hline C & 1.681400 & 1.830900 & 1.783600 \\
\hline C & 0.111900 & 1.770000 & 2.017600 \\
\hline
\end{tabular}




$\begin{array}{lrrr}\mathrm{C} & -0.363200 & 3.123500 & 2.499600 \\ \mathrm{H} & -1.307100 & 3.176000 & 3.073200 \\ \mathrm{C} & 2.323400 & 3.090500 & 1.436800 \\ \mathrm{C} & 1.634000 & 4.271100 & 1.580400 \\ \mathrm{C} & 0.318000 & 4.273500 & 2.225000 \\ \mathrm{H} & -0.110400 & 5.242900 & 2.532000 \\ \mathrm{H} & 3.394300 & 3.123800 & 1.167200 \\ \mathrm{H} & 2.111800 & 5.233000 & 1.331500 \\ \mathrm{~K} & -1.960700 & -1.069000 & -1.975000 \\ \mathrm{H} & -2.831000 & -0.953400 & -5.991600 \\ \mathrm{C} & -3.389800 & -1.487200 & -5.206600 \\ \mathrm{C} & -4.416600 & -0.828400 & -4.502000 \\ \mathrm{C} & -3.083700 & -2.830800 & -4.913000 \\ \mathrm{C} & -5.138800 & -1.514800 & -3.506400 \\ \mathrm{H} & -4.656500 & 0.221600 & -4.730900 \\ \mathrm{C} & -3.805100 & -3.516600 & -3.915800 \\ \mathrm{H} & -2.286200 & -3.348100 & -5.469600 \\ \mathrm{C} & -4.833700 & -2.858300 & -3.212900 \\ \mathrm{H} & -5.945300 & -1.001200 & -2.959900 \\ \mathrm{H} & -3.571300 & -4.569700 & -3.693400 \\ \mathrm{H} & -5.404800 & -3.396000 & -2.439400\end{array}$

\section{Table S26. Cartesian coordinates of int-6*}

Zero-point correction $=$

Thermal correction to Energy=

Thermal correction to Enthalpy=

Thermal correction to Gibbs Free Energy=

Sum of electronic and zero-point Energies= -5209.566729

Sum of electronic and thermal Energies= $\quad-5209.488431$

Sum of electronic and thermal Enthalpies $=-5209.487486$

Sum of electronic and thermal Free Energies $=-5209.678156$

1.310959

1.311903

1.121233
1.232660 (Hartree/Particle)

$-0.736400-1.052800$

$0.974100 \quad 0.085200$

$-3.809600-1.991000$

$-2.755800-2.165100$

$-4.351000-1.553300$

$-4.263600-2.978900$

$-3.649500 \quad 0.956700$

$-3.926000 \quad 1.679400$

$-4.281800 \quad 1.199900$

$-2.586400 \quad 1.097900$

$-5.819000-0.851400$

$-6.202200-1.875800$

$-6.414500-0.426000$

$-6.033000-0.240800$

$-3.880300-4.366400$

$-4.865000-4.135600$

$-3.998600 \quad-5.314000$

$-3.157400-4.545700$

$-2.009700 \quad-3.721800$

$-1.037300-3.927900$

$-2.383000-4.677400$

$-1.820500 \quad-3.027700$

$-4.841700-2.764500$

$-4.588200-2.139600$

$-5.157500 \quad-3.762500$

$-5.714700-2.305000$

$-2.800800-1.312200$

$-2.974100-0.357300$

$-3.971900-0.040200$

$-1.880700 \quad-0.013800$

$\begin{array}{ll}-2.027400 & 0.555200\end{array}$ 


\begin{tabular}{|c|c|c|c|}
\hline C & -3.871000 & 0.442500 & -0.257900 \\
\hline C & -3.284300 & 1.827300 & -0.589800 \\
\hline $\mathrm{H}$ & -2.817800 & 1.793000 & -1.594900 \\
\hline $\mathrm{H}$ & -2.467400 & 2.052100 & 0.126800 \\
\hline C & -5.020700 & 0.159100 & -1.271400 \\
\hline $\mathrm{H}$ & -5.462500 & -0.840800 & -1.064400 \\
\hline $\mathrm{H}$ & -4.585000 & 0.109500 & -2.291300 \\
\hline C & -4.512500 & 0.538800 & 1.165000 \\
\hline $\mathrm{H}$ & -3.714900 & 0.768200 & 1.906900 \\
\hline $\mathrm{H}$ & -4.962500 & -0.436200 & 1.454000 \\
\hline C & -4.363400 & 2.933000 & -0.542300 \\
\hline $\mathrm{H}$ & -3.891400 & 3.906900 & -0.796300 \\
\hline C & -5.480600 & 2.614900 & -1.561300 \\
\hline $\mathrm{H}$ & -5.062700 & 2.592000 & -2.591800 \\
\hline $\mathrm{H}$ & -6.253700 & 3.415700 & -1.544800 \\
\hline C & -6.114000 & 1.249100 & -1.215200 \\
\hline $\mathrm{H}$ & -6.912300 & 1.008200 & -1.952300 \\
\hline C & -6.718500 & 1.304500 & 0.205200 \\
\hline $\mathrm{H}$ & -7.520400 & 2.074600 & 0.254700 \\
\hline $\mathrm{H}$ & -7.192600 & 0.329600 & 0.458400 \\
\hline C & -5.603700 & 1.635000 & 1.222200 \\
\hline $\mathrm{H}$ & -6.032900 & 1.670000 & 2.249000 \\
\hline C & -4.975400 & 3.002000 & 0.874100 \\
\hline $\mathrm{H}$ & -5.746600 & 3.803300 & 0.923500 \\
\hline $\mathrm{H}$ & -4.193100 & 3.264200 & 1.621000 \\
\hline C & 1.321600 & 0.162000 & 3.428500 \\
\hline $\mathrm{H}$ & 2.391600 & 0.242700 & 3.707700 \\
\hline $\mathrm{H}$ & 0.794000 & -0.284300 & 4.301600 \\
\hline $\mathrm{H}$ & 1.266500 & -0.520000 & 2.557400 \\
\hline C & -1.267600 & 1.855700 & 3.048600 \\
\hline $\mathrm{H}$ & -1.648200 & 1.466000 & 2.082300 \\
\hline $\mathrm{H}$ & -1.678300 & 1.247400 & 3.885700 \\
\hline $\mathrm{H}$ & -1.691000 & 2.874700 & 3.167500 \\
\hline C & 1.117500 & 2.842400 & 4.674900 \\
\hline $\mathrm{H}$ & 0.641000 & 3.835500 & 4.780100 \\
\hline $\mathrm{H}$ & 0.798500 & 2.224400 & 5.542700 \\
\hline $\mathrm{H}$ & 2.216300 & 2.974700 & 4.754300 \\
\hline C & 1.870200 & 5.479800 & 0.062300 \\
\hline $\mathrm{H}$ & 1.872300 & 5.248000 & -1.023200 \\
\hline $\mathrm{H}$ & 1.509500 & 6.527400 & 0.135900 \\
\hline $\mathrm{H}$ & 2.903600 & 5.480700 & 0.474900 \\
\hline C & -0.997300 & 4.436500 & 0.167300 \\
\hline $\mathrm{H}$ & -1.811900 & 4.020200 & 0.792900 \\
\hline $\mathrm{H}$ & -1.259900 & 5.491900 & -0.059400 \\
\hline $\mathrm{H}$ & -0.974300 & 3.878700 & -0.790200 \\
\hline C & 0.411100 & 5.466700 & 2.641400 \\
\hline $\mathrm{H}$ & 1.330400 & 5.557300 & 3.256000 \\
\hline $\mathrm{H}$ & 0.123200 & 6.490700 & 2.318900 \\
\hline $\mathrm{H}$ & -0.400800 & 5.093300 & 3.298200 \\
\hline C & 1.367100 & 2.641800 & 1.444800 \\
\hline C & 2.836900 & 2.770100 & 1.831900 \\
\hline $\mathrm{H}$ & 3.210000 & 3.562800 & 2.507000 \\
\hline C & 3.700700 & 1.764300 & 1.475400 \\
\hline $\mathrm{H}$ & 4.742900 & 1.792900 & 1.847800 \\
\hline C & 4.347300 & -0.353000 & 0.509000 \\
\hline C & 5.617300 & 0.173600 & -0.235300 \\
\hline $\mathrm{H}$ & 6.050900 & 1.037700 & 0.318100 \\
\hline $\mathrm{H}$ & 5.314300 & 0.538400 & -1.244900 \\
\hline C & 4.808500 & -0.906300 & 1.895800 \\
\hline $\mathrm{H}$ & 3.917500 & -1.303000 & 2.429000 \\
\hline $\mathrm{H}$ & 5.214800 & -0.084400 & 2.524000 \\
\hline C & 3.793700 & -1.550400 & -0.294400 \\
\hline $\mathrm{H}$ & 2.901300 & -1.951700 & 0.228700 \\
\hline $\mathrm{H}$ & 3.450500 & -1.202200 & -1.290400 \\
\hline C & 6.688400 & -0.929100 & -0.391600 \\
\hline $\mathrm{H}$ & 7.572900 & -0.511700 & -0.923400 \\
\hline C & 7.112400 & -1.432200 & 1.006400 \\
\hline $\mathrm{H}$ & 7.901700 & -2.210400 & 0.909000 \\
\hline
\end{tabular}




\begin{tabular}{|c|c|c|c|}
\hline $\mathrm{H}$ & 7.556700 & -0.598800 & 1.595900 \\
\hline C & 5.881100 & -2.007900 & 1.741900 \\
\hline $\mathrm{H}$ & 6.184200 & -2.364200 & 2.751700 \\
\hline C & 5.295400 & -3.180300 & 0.927000 \\
\hline $\mathrm{H}$ & 6.049700 & -3.991600 & 0.816800 \\
\hline $\mathrm{H}$ & 4.424800 & -3.620300 & 1.461700 \\
\hline C & 4.859900 & -2.659400 & -0.459300 \\
\hline $\mathrm{H}$ & 4.420000 & -3.489300 & -1.050900 \\
\hline C & 6.090300 & -2.097600 & -1.204600 \\
\hline $\mathrm{H}$ & 6.852700 & -2.895400 & -1.348600 \\
\hline $\mathrm{H}$ & 5.796600 & -1.748200 & -2.219300 \\
\hline $\mathrm{N}$ & -2.806300 & -0.582900 & -0.335500 \\
\hline $\mathrm{N}$ & 3.318400 & 0.711500 & 0.651500 \\
\hline 0 & 0.154900 & -0.267100 & 0.310000 \\
\hline Si & 0.158100 & -3.960600 & -0.846500 \\
\hline Si & -2.070600 & -3.337500 & -2.986200 \\
\hline $\mathrm{Si}$ & 0.643600 & 1.916400 & 3.070100 \\
\hline $\mathrm{Si}$ & 0.657300 & 4.382200 & 1.083400 \\
\hline K & 3.613500 & 3.195900 & -1.092800 \\
\hline C & 1.371100 & -0.668100 & -4.380600 \\
\hline C & 2.605900 & -0.148000 & -3.803900 \\
\hline C & 0.164600 & -0.418400 & -3.800700 \\
\hline C & 2.596600 & 0.690400 & -2.729800 \\
\hline $\mathrm{H}$ & 3.592000 & 0.959000 & -2.324100 \\
\hline C & -0.043800 & 0.402200 & -2.559900 \\
\hline C & 1.335900 & 1.169400 & -2.045600 \\
\hline $\mathrm{H}$ & 3.568800 & -0.472200 & -4.235700 \\
\hline $\mathrm{H}$ & 1.429200 & -1.288000 & -5.290000 \\
\hline $\mathrm{H}$ & -0.741000 & -0.826300 & -4.280300 \\
\hline C & -0.506700 & 1.787300 & -2.998900 \\
\hline C & 0.715900 & 2.424700 & -2.666300 \\
\hline C & 0.962700 & 3.742300 & -3.080900 \\
\hline $\mathrm{H}$ & 1.932800 & 4.262300 & -2.972500 \\
\hline C & -1.529700 & 2.445800 & -3.687700 \\
\hline C & -1.302300 & 3.798900 & -4.041600 \\
\hline C & -0.077400 & 4.429000 & -3.757500 \\
\hline $\mathrm{H}$ & 0.086200 & 5.468200 & -4.086000 \\
\hline $\mathrm{H}$ & -2.458600 & 1.938200 & -3.995000 \\
\hline $\mathrm{H}$ & -2.082600 & 4.358500 & -4.582500 \\
\hline K & -1.332800 & -1.359100 & 2.260600 \\
\hline $\mathrm{H}$ & -0.162700 & -3.288400 & 5.758900 \\
\hline C & -1.176800 & -3.127800 & 5.359900 \\
\hline C & -1.919600 & -1.998100 & 5.756100 \\
\hline C & -1.734500 & -4.057300 & 4.459900 \\
\hline C & -3.220500 & -1.798600 & 5.252300 \\
\hline $\mathrm{H}$ & -1.487500 & -1.276300 & 6.467300 \\
\hline C & -3.034100 & -3.856700 & 3.954000 \\
\hline $\mathrm{H}$ & -1.156300 & -4.943700 & 4.154700 \\
\hline $\mathrm{H}$ & -3.805500 & -0.920600 & 5.569200 \\
\hline C & -3.777300 & -2.727700 & 4.351500 \\
\hline $\mathrm{H}$ & -3.469300 & -4.583000 & 3.250000 \\
\hline $\mathrm{H}$ & -4.795800 & -2.574200 & 3.961500 \\
\hline
\end{tabular}

\section{Table S27. Cartesian coordinates of 2*-isomer1}

Zero-point correction=

Thermal correction to Energy=

Thermal correction to Enthalpy=

Thermal correction to Gibbs Free Energy=

Sum of electronic and zero-point Energies=

Sum of electronic and thermal Energies=

Sum of electronic and thermal Enthalpies=

Sum of electronic and thermal Free Energies $=-4516.111330$

\subsection{9 (Hartree/Particle)}

1.040287

1.041231

0.883962

$-4516.018073$

$-4515.955005$

$-4515.954061$
Al
0.627900
1.076300
0.027800 


\begin{tabular}{|c|c|c|c|}
\hline Al & -0.627900 & -1.076200 & 0.028000 \\
\hline C & -2.456400 & 2.992600 & 1.803900 \\
\hline $\mathrm{H}$ & -2.561200 & 2.099700 & 1.153200 \\
\hline $\mathrm{H}$ & -2.978400 & 2.822800 & 2.773500 \\
\hline $\mathrm{H}$ & -3.005700 & 3.809700 & 1.294000 \\
\hline C & -0.638800 & 5.290500 & 2.629300 \\
\hline $\mathrm{H}$ & -1.109100 & 5.949600 & 1.870400 \\
\hline $\mathrm{H}$ & -1.218000 & 5.401900 & 3.572100 \\
\hline $\mathrm{H}$ & 0.385100 & 5.675500 & 2.819100 \\
\hline C & -0.031900 & 2.497400 & 3.621600 \\
\hline $\mathrm{H}$ & 0.997200 & 2.808500 & 3.905900 \\
\hline $\mathrm{H}$ & -0.685800 & 2.689600 & 4.500100 \\
\hline $\mathrm{H}$ & -0.036400 & 1.411700 & 3.381000 \\
\hline C & -1.704300 & 4.478700 & -1.277600 \\
\hline $\mathrm{H}$ & -2.215300 & 5.072800 & -0.491400 \\
\hline $\mathrm{H}$ & -1.825000 & 5.028900 & -2.235500 \\
\hline $\mathrm{H}$ & -2.237000 & 3.511000 & -1.379400 \\
\hline C & 0.913200 & 5.970200 & -0.719800 \\
\hline $\mathrm{H}$ & 2.014300 & 5.912900 & -0.592400 \\
\hline $\mathrm{H}$ & 0.712100 & 6.579600 & -1.627300 \\
\hline $\mathrm{H}$ & 0.500400 & 6.518300 & 0.151700 \\
\hline C & 0.918400 & 3.508100 & -2.506800 \\
\hline $\mathrm{H}$ & 0.513500 & 2.498700 & -2.733100 \\
\hline $\mathrm{H}$ & 0.708700 & 4.167900 & -3.376300 \\
\hline $\mathrm{H}$ & 2.019000 & 3.419200 & -2.405400 \\
\hline C & 0.497700 & 3.107200 & 0.591900 \\
\hline C & 1.946300 & 3.351900 & 0.998400 \\
\hline $\mathrm{H}$ & 2.264800 & 4.287700 & 1.496100 \\
\hline C & 2.919000 & 2.444700 & 0.660200 \\
\hline $\mathrm{H}$ & 3.982900 & 2.681400 & 0.871600 \\
\hline C & 3.683000 & 0.594600 & -0.746600 \\
\hline C & 4.828100 & 0.048400 & 0.166400 \\
\hline $\mathrm{H}$ & 4.401500 & -0.704200 & 0.870800 \\
\hline $\mathrm{H}$ & 5.257000 & 0.876800 & 0.775500 \\
\hline C & 4.323400 & 1.591000 & -1.762100 \\
\hline $\mathrm{H}$ & 4.731100 & 2.471900 & -1.221000 \\
\hline $\mathrm{H}$ & 3.520100 & 1.971600 & -2.428300 \\
\hline C & 3.125800 & -0.595400 & -1.562800 \\
\hline $\mathrm{H}$ & 2.652400 & -1.328300 & -0.872100 \\
\hline $\mathrm{H}$ & 2.301800 & -0.233800 & -2.215000 \\
\hline C & 5.947600 & -0.623200 & -0.664300 \\
\hline $\mathrm{H}$ & 6.742400 & -0.996100 & 0.020300 \\
\hline C & 6.547800 & 0.407400 & -1.645700 \\
\hline $\mathrm{H}$ & 6.995800 & 1.257000 & -1.083200 \\
\hline $\mathrm{H}$ & 7.370800 & -0.060000 & -2.230800 \\
\hline C & 5.440700 & 0.921800 & -2.592900 \\
\hline $\mathrm{H}$ & 5.870300 & 1.668300 & -3.297400 \\
\hline C & 4.845100 & -0.261900 & -3.386400 \\
\hline $\mathrm{H}$ & 5.632000 & -0.751100 & -4.003200 \\
\hline $\mathrm{H}$ & 4.064400 & 0.103300 & -4.089300 \\
\hline C & 4.235400 & -1.277800 & -2.395900 \\
\hline $\mathrm{H}$ & 3.791200 & -2.128200 & -2.958100 \\
\hline C & 5.344800 & -1.804200 & -1.458000 \\
\hline $\mathrm{H}$ & 6.139000 & -2.312600 & -2.049000 \\
\hline $\mathrm{H}$ & 4.928300 & -2.563800 & -0.759700 \\
\hline C & 0.031700 & -2.496600 & 3.622000 \\
\hline $\mathrm{H}$ & -0.997500 & -2.807600 & 3.906300 \\
\hline $\mathrm{H}$ & 0.685500 & -2.688500 & 4.500600 \\
\hline $\mathrm{H}$ & 0.036200 & -1.410900 & 3.381200 \\
\hline C & 2.456300 & -2.992100 & 1.804700 \\
\hline $\mathrm{H}$ & 2.561100 & -2.099400 & 1.153800 \\
\hline $\mathrm{H}$ & 2.978200 & -2.822100 & 2.774300 \\
\hline $\mathrm{H}$ & 3.005600 & -3.809300 & 1.295100 \\
\hline C & 0.638700 & -5.289900 & 2.630400 \\
\hline $\mathrm{H}$ & 1.109000 & -5.949100 & 1.871700 \\
\hline $\mathrm{H}$ & 1.217700 & -5.401100 & 3.573200 \\
\hline $\mathrm{H}$ & -0.385200 & -5.674900 & 2.820200 \\
\hline C & -0.918000 & -3.508600 & -2.506200 \\
\hline
\end{tabular}




\begin{tabular}{|c|c|c|c|}
\hline $\mathrm{H}$ & -0.513200 & -2.499200 & -2.732600 \\
\hline $\mathrm{H}$ & -0.708100 & -4.168500 & -3.375500 \\
\hline $\mathrm{H}$ & -2.018600 & -3.419700 & -2.405100 \\
\hline C & 1.704500 & -4.479000 & -1.276500 \\
\hline $\mathrm{H}$ & 2.215400 & -5.073000 & -0.490100 \\
\hline $\mathrm{H}$ & 1.825300 & -5.029300 & -2.234300 \\
\hline $\mathrm{H}$ & 2.237200 & -3.511300 & -1.378400 \\
\hline C & -0.913200 & -5.970300 & -0.718700 \\
\hline $\mathrm{H}$ & -2.014200 & -5.912900 & -0.591400 \\
\hline $\mathrm{H}$ & -0.711900 & -6.579800 & -1.626100 \\
\hline $\mathrm{H}$ & -0.500500 & -6.518200 & 0.153000 \\
\hline C & -0.497700 & -3.107000 & 0.592400 \\
\hline C & -1.946300 & -3.351600 & 0.998900 \\
\hline $\mathrm{H}$ & -2.264900 & -4.287400 & 1.496800 \\
\hline C & -2.919000 & -2.444500 & 0.660600 \\
\hline $\mathrm{H}$ & -3.982900 & -2.681200 & 0.872000 \\
\hline C & -3.683000 & -0.594800 & -0.746700 \\
\hline C & -4.323100 & -1.591400 & -1.762000 \\
\hline $\mathrm{H}$ & -4.730700 & -2.472300 & -1.220900 \\
\hline $\mathrm{H}$ & -3.519700 & -1.972100 & -2.428100 \\
\hline C & -3.125800 & 0.595100 & -1.563000 \\
\hline $\mathrm{H}$ & -2.301600 & 0.233600 & -2.215100 \\
\hline $\mathrm{H}$ & -2.652700 & 1.328300 & -0.872400 \\
\hline C & -4.828300 & -0.048500 & 0.166100 \\
\hline $\mathrm{H}$ & -4.401900 & 0.704300 & 0.870300 \\
\hline $\mathrm{H}$ & -5.257200 & -0.876800 & 0.775200 \\
\hline C & -5.440300 & -0.922500 & -2.593200 \\
\hline $\mathrm{H}$ & -5.869700 & -1.669200 & -3.297600 \\
\hline C & -4.844800 & 0.261100 & -3.386800 \\
\hline $\mathrm{H}$ & -4.064000 & -0.104100 & -4.089500 \\
\hline $\mathrm{H}$ & -5.631700 & 0.750000 & -4.003800 \\
\hline C & -4.235500 & 1.277200 & -2.396400 \\
\hline $\mathrm{H}$ & -3.791200 & 2.127600 & -2.958700 \\
\hline C & -5.345100 & 1.803700 & -1.458700 \\
\hline $\mathrm{H}$ & -6.139200 & 2.311900 & -2.050000 \\
\hline $\mathrm{H}$ & -4.928800 & 2.563500 & -0.760500 \\
\hline C & -5.947700 & 0.622700 & -0.665000 \\
\hline $\mathrm{H}$ & -6.742700 & 0.995600 & 0.019500 \\
\hline C & -6.547600 & -0.408100 & -1.646300 \\
\hline $\mathrm{H}$ & -7.370600 & 0.059000 & -2.231600 \\
\hline $\mathrm{H}$ & -6.995700 & -1.257700 & -1.083600 \\
\hline K & 2.337000 & 0.219600 & 2.717800 \\
\hline K & -2.337200 & -0.218900 & 2.717700 \\
\hline$N$ & 2.602900 & 1.228900 & 0.045000 \\
\hline $\mathrm{N}$ & -2.602900 & -1.228800 & 0.045200 \\
\hline 0 & -0.000000 & 0.000200 & 1.426400 \\
\hline $\mathrm{Si}$ & -0.616500 & 3.464800 & 2.067200 \\
\hline $\mathrm{Si}$ & 0.154200 & 4.230300 & -0.922200 \\
\hline Si & 0.616300 & -3.464300 & 2.068000 \\
\hline Si & -0.154000 & -4.230500 & -0.921400 \\
\hline
\end{tabular}

Table S28. Cartesian coordinates of $\mathbf{2}^{*}$-isomer2

Zero-point correction=

Thermal correction to Energy=

Thermal correction to Enthalpy=

Thermal correction to Gibbs Free Energy=

Sum of electronic and zero-point Energies=

Sum of electronic and thermal Energies=

Sum of electronic and thermal Enthalpies=

Sum of electronic and thermal Free Energies= -4516.105951

\subsection{0 (Hartree/Particle)}

1.040762

1.041706

0.881238

$-4516.009948$

$-4515.946427$

$-4515.945483$
Al $\quad-0.579900 \quad-1.102300$
0.086600
Al
0.799500
1.182700
0.273700 


\begin{tabular}{|c|c|c|c|}
\hline C & 2.580500 & -2.851300 & 1.999300 \\
\hline $\mathrm{H}$ & 2.640100 & -1.748100 & 1.908100 \\
\hline $\mathrm{H}$ & 3.156700 & -3.152300 & 2.900700 \\
\hline $\mathrm{H}$ & 3.074500 & -3.305400 & 1.117500 \\
\hline C & 0.150600 & -2.467200 & 3.741600 \\
\hline $\mathrm{H}$ & -0.916400 & -2.694600 & 3.959600 \\
\hline $\mathrm{H}$ & 0.737700 & -2.773800 & 4.632800 \\
\hline $\mathrm{H}$ & 0.314100 & -1.380400 & 3.585500 \\
\hline C & 0.839300 & -5.238600 & 2.723200 \\
\hline $\mathrm{H}$ & 1.426500 & -5.850500 & 2.007100 \\
\hline $\mathrm{H}$ & 1.335100 & -5.325300 & 3.714400 \\
\hline $\mathrm{H}$ & -0.167300 & -5.698200 & 2.810400 \\
\hline C & 1.792800 & -4.194500 & -1.390800 \\
\hline $\mathrm{H}$ & 2.489000 & -4.670000 & -0.669000 \\
\hline $\mathrm{H}$ & 1.899800 & -4.734200 & -2.356500 \\
\hline $\mathrm{H}$ & 2.130500 & -3.147700 & -1.539500 \\
\hline C & -1.108700 & -3.818000 & -2.292300 \\
\hline $\mathrm{H}$ & -0.911200 & -2.790500 & -2.667300 \\
\hline $\mathrm{H}$ & -0.928400 & -4.532500 & -3.124600 \\
\hline $\mathrm{H}$ & -2.183100 & -3.869500 & -2.023200 \\
\hline C & -0.430000 & -6.097800 & -0.461200 \\
\hline $\mathrm{H}$ & -1.471000 & -6.213500 & -0.093200 \\
\hline $\mathrm{H}$ & -0.345500 & -6.675000 & -1.407500 \\
\hline $\mathrm{H}$ & 0.244700 & -6.568200 & 0.281500 \\
\hline C & -0.366100 & -3.109900 & 0.692300 \\
\hline C & -1.795600 & -3.414100 & 1.118100 \\
\hline $\mathrm{H}$ & -2.067600 & -4.366400 & 1.613100 \\
\hline C & -2.807100 & -2.532200 & 0.839000 \\
\hline $\mathrm{H}$ & -3.852600 & -2.813800 & 1.079000 \\
\hline C & -3.708700 & -0.680700 & -0.463900 \\
\hline C & -3.309900 & 0.681500 & -1.074800 \\
\hline $\mathrm{H}$ & -2.428400 & 0.527100 & -1.736300 \\
\hline $\mathrm{H}$ & -2.958300 & 1.359200 & -0.265700 \\
\hline C & -4.189900 & -1.592900 & -1.633600 \\
\hline $\mathrm{H}$ & -4.480300 & -2.587800 & -1.231500 \\
\hline $\mathrm{H}$ & -3.327700 & -1.766800 & -2.313000 \\
\hline C & -4.933500 & -0.419900 & 0.472700 \\
\hline $\mathrm{H}$ & -4.619200 & 0.267600 & 1.294000 \\
\hline $\mathrm{H}$ & -5.266600 & -1.368000 & 0.947900 \\
\hline C & -4.478500 & 1.327100 & -1.853100 \\
\hline $\mathrm{H}$ & -4.141400 & 2.300400 & -2.272600 \\
\hline C & -4.920600 & 0.390900 & -2.999500 \\
\hline $\mathrm{H}$ & -4.080000 & 0.233700 & -3.713100 \\
\hline $\mathrm{H}$ & -5.749400 & 0.857000 & -3.577900 \\
\hline C & -5.368900 & -0.963100 & -2.406300 \\
\hline $\mathrm{H}$ & -5.682000 & -1.647100 & -3.226500 \\
\hline C & -6.552800 & -0.738500 & -1.439900 \\
\hline $\mathrm{H}$ & -7.420800 & -0.304500 & -1.984200 \\
\hline $\mathrm{H}$ & -6.892000 & -1.711500 & -1.019600 \\
\hline C & -6.116100 & 0.210800 & -0.301000 \\
\hline $\mathrm{H}$ & -6.967300 & 0.371500 & 0.397700 \\
\hline C & -5.670500 & 1.562900 & -0.899200 \\
\hline $\mathrm{H}$ & -6.513900 & 2.038400 & -1.447700 \\
\hline $\mathrm{H}$ & -5.378400 & 2.266000 & -0.087900 \\
\hline C & 0.528400 & 2.424100 & 3.651000 \\
\hline $\mathrm{H}$ & 1.587000 & 2.748500 & 3.726500 \\
\hline $\mathrm{H}$ & 0.064200 & 2.550400 & 4.653700 \\
\hline $\mathrm{H}$ & 0.537800 & 1.349800 & 3.368500 \\
\hline C & -2.231000 & 2.969300 & 2.345800 \\
\hline $\mathrm{H}$ & -2.451700 & 2.134100 & 1.648800 \\
\hline $\mathrm{H}$ & -2.561900 & 2.703500 & 3.376100 \\
\hline $\mathrm{H}$ & -2.868100 & 3.818400 & 2.024400 \\
\hline C & -0.321700 & 5.262200 & 2.922200 \\
\hline $\mathrm{H}$ & -0.901500 & 5.938800 & 2.259800 \\
\hline $\mathrm{H}$ & -0.752900 & 5.344100 & 3.943600 \\
\hline $\mathrm{H}$ & 0.719400 & 5.643800 & 2.969100 \\
\hline C & 0.303100 & 3.549300 & -2.493100 \\
\hline $\mathrm{H}$ & -0.408600 & 2.749400 & -2.793300 \\
\hline
\end{tabular}




\begin{tabular}{|c|c|c|c|}
\hline $\mathrm{H}$ & 0.285500 & 4.328400 & -3.285700 \\
\hline $\mathrm{H}$ & 1.332100 & 3.134000 & -2.430900 \\
\hline C & -2.125600 & 4.429500 & -0.847600 \\
\hline $\mathrm{H}$ & -2.502000 & 5.063700 & -0.017800 \\
\hline $\mathrm{H}$ & -2.436400 & 4.913600 & -1.798600 \\
\hline $\mathrm{H}$ & -2.636200 & 3.448100 & -0.781800 \\
\hline C & 0.446100 & 6.049600 & -0.761800 \\
\hline $\mathrm{H}$ & 1.537100 & 6.076800 & -0.959200 \\
\hline $\mathrm{H}$ & -0.058400 & 6.656400 & -1.544500 \\
\hline $\mathrm{H}$ & 0.268400 & 6.544500 & 0.215200 \\
\hline C & 0.461300 & 3.205700 & 0.632700 \\
\hline C & 1.908600 & 3.666200 & 0.774200 \\
\hline $\mathrm{H}$ & 2.164700 & 4.691900 & 1.092600 \\
\hline C & 2.927200 & 2.804900 & 0.481100 \\
\hline $\mathrm{H}$ & 3.973400 & 3.151400 & 0.568500 \\
\hline C & 3.887200 & 0.763600 & -0.415600 \\
\hline C & 4.555100 & 1.511500 & -1.611900 \\
\hline $\mathrm{H}$ & 4.836200 & 2.541500 & -1.306600 \\
\hline $\mathrm{H}$ & 3.798200 & 1.624100 & -2.423300 \\
\hline C & 4.959100 & 0.585800 & 0.706700 \\
\hline $\mathrm{H}$ & 4.495100 & 0.033400 & 1.552400 \\
\hline $\mathrm{H}$ & 5.258200 & 1.580400 & 1.101200 \\
\hline C & 3.510600 & -0.646700 & -0.919100 \\
\hline $\mathrm{H}$ & 3.001500 & -1.208600 & -0.110500 \\
\hline $\mathrm{H}$ & 2.758300 & -0.547000 & -1.733000 \\
\hline C & 5.799700 & 0.757400 & -2.132600 \\
\hline $\mathrm{H}$ & 6.250100 & 1.326700 & -2.976400 \\
\hline C & 6.831800 & 0.622500 & -0.989200 \\
\hline $\mathrm{H}$ & 7.744800 & 0.103200 & -1.357300 \\
\hline $\mathrm{H}$ & 7.153500 & 1.630800 & -0.645500 \\
\hline C & 6.205200 & -0.165500 & 0.183200 \\
\hline $\mathrm{H}$ & 6.947600 & -0.263200 & 1.006200 \\
\hline C & 5.783300 & -1.568000 & -0.304800 \\
\hline $\mathrm{H}$ & 6.668400 & -2.135700 & -0.670400 \\
\hline $\mathrm{H}$ & 5.349900 & -2.151200 & 0.536500 \\
\hline C & 4.744700 & -1.419900 & -1.438500 \\
\hline $\mathrm{H}$ & 4.421400 & -2.427700 & -1.780200 \\
\hline C & 5.380400 & -0.648100 & -2.615600 \\
\hline $\mathrm{H}$ & 6.260200 & -1.203100 & -3.011100 \\
\hline $\mathrm{H}$ & 4.653900 & -0.561200 & -3.456800 \\
\hline $\mathrm{N}$ & -2.563900 & -1.281300 & 0.256700 \\
\hline $\mathrm{N}$ & 2.698000 & 1.488900 & 0.075200 \\
\hline 0 & 0.007100 & 0.034700 & 1.389100 \\
\hline Si & 0.772900 & -3.402300 & 2.190600 \\
\hline Si & -0.016700 & -4.261600 & -0.788300 \\
\hline Si & -0.368000 & 3.450200 & 2.319300 \\
\hline $\mathrm{Si}$ & -0.225200 & 4.263500 & -0.790900 \\
\hline K & -2.056200 & -0.285500 & 2.951300 \\
\hline $\mathrm{K}$ & 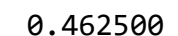 & 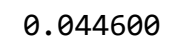 & -3.2 \\
\hline
\end{tabular}

Table S29. Cartesian coordinates of 2_K-free

Zero-point correction=

Thermal correction to Energy=

Thermal correction to Enthalpy=

Thermal correction to Gibbs Free Energy=

Sum of electronic and zero-point Energies=

Sum of electronic and thermal Energies=

Sum of electronic and thermal Enthalpies=

Sum of electronic and thermal Free Energies=
0.972177 (Hartree/Particle)

1.031731

1.032675

0.883485

$-3316.016170$

$-3315.956616$

$-3315.955672$

$-3316.104862$

$\begin{array}{lrrr}\text { Al } & -0.657500 & -1.089600 & 0.144600 \\ \text { Al } & 0.657500 & 1.089600 & 0.144600 \\ \text { C } & 2.386200 & -2.526800 & 2.145300 \\ \text { H } & 2.267000 & -1.445500 & 1.923000 \\ \text { H } & 2.920200 & -2.635400 & 3.115300\end{array}$




\begin{tabular}{|c|c|c|}
\hline 3.012600 & -2.988400 & 1.353900 \\
\hline-0.282500 & -2.418000 & 3.643000 \\
\hline-1.302300 & -2.836300 & 3.775200 \\
\hline 0.266900 & -2.500600 & 4.606900 \\
\hline-0.369300 & -1.346700 & 3.356000 \\
\hline 0.891900 & -5.119000 & 2.780800 \\
\hline 1.571900 & -5.668500 & 2.093800 \\
\hline 1.336400 & -5.166200 & 3.801000 \\
\hline-0.072800 & -5.670800 & 2.809600 \\
\hline 2.068600 & -4.141900 & -1.239500 \\
\hline 2.665700 & -4.600900 & -0.422600 \\
\hline 2.294400 & -4.694400 & -2.178500 \\
\hline 2.412600 & -3.095600 & -1.363200 \\
\hline-0.707000 & -3.638800 & -2.449800 \\
\hline-0.410200 & -2.604800 & -2.727800 \\
\hline-0.487600 & -4.319400 & -3.303200 \\
\hline-1.803400 & -3.634400 & -2.274500 \\
\hline-0.226600 & -6.054100 & -0.675000 \\
\hline-1.318600 & -6.195800 & -0.530800 \\
\hline 0.072100 & -6.613400 & -1.590200 \\
\hline 0.291300 & -6.516400 & 0.19200 \\
\hline-0.348200 & -3.152700 & 3906 \\
\hline-1.775100 & -3.604200 & 0.90540 \\
\hline-2.012000 & -4.587500 & 1.358306 \\
\hline-2.811600 & -2.761200 & 0.60400 \\
\hline-3.853200 & -3. & 0.801600 \\
\hline-3.789600 & 1700 & -0.46660 \\
\hline-3.398800 & 0.506600 & -1.151800 \\
\hline-2.651400 & 0.301800 & -1.948600 \\
\hline-2.875300 & 1.147500 & -0.412000 \\
\hline-4.523900 & -1.694500 & -1.541000 \\
\hline-4.816300 & -2.670100 & -1.095106 \\
\hline-3.800100 & -1.921300 & -2.35470 \\
\hline-4.821600 & -0.485100 & 0.65900 \\
\hline-4.312000 & 0. & 1.414900 \\
\hline-5.1 & -1.2 & 6900 \\
\hline-4.638100 & 1.2 & -1.723200 \\
\hline-4.3 & 2.1 & -2.200400 \\
\hline-5.339000 & 0.344100 & -2.772700 \\
\hline-4.645300 & 0.132700 & -3.617100 \\
\hline-6.223800 & 0.871600 & -3.202100 \\
\hline-5.772500 & -0.978600 & -2.103900 \\
\hline-6.273000 & -1.633400 & -2.856200 \\
\hline-6.758300 & 2700 & -0.951900 \\
\hline-7.673700 & -0 & -1 \\
\hline-7.0 & $-1 . \epsilon$ & -0.473400 \\
\hline-6.0 & $\partial 0$ & 0.09340 \\
\hline-6.7 & $\theta 0$ & 0.921400 \\
\hline-5.631500 & 1.552000 & -0.582800 \\
\hline-6.520500 & 2.095400 & -0.982900 \\
\hline-5.149300 & 2.219800 & 0.163800 \\
\hline 0.282500 & 2.418000 & 3.64300 \\
\hline 1.302200 & 300 & 3.775200 \\
\hline-0.267000 & $\theta 0$ & 4.60680 \\
\hline 0.36 & 700 & 3.35600 \\
\hline-2.3 & $\theta 0$ & 2.14520 \\
\hline-2.26 & $\partial 0$ & 1.92290 \\
\hline-2.920200 & .635400 & 3.11530 \\
\hline-3.012600 & 2.988400 & 1.35380 \\
\hline-0.891900 & 5.119000 & 2.78080 \\
\hline-1.571900 & 5.668500 & 2.09370 \\
\hline-1.336400 & 5.166300 & 3.80100 \\
\hline 0.072700 & 5.670900 & 2.80960 \\
\hline 0.707100 & 3.638800 & -2.44990 \\
\hline 0.410200 & 2.604700 & -2.72780 \\
\hline 0.487600 & 4.319400 & -3.30320 \\
\hline 1.803500 & 3.634400 & -2.27450 \\
\hline-2.068606 & 4.141900 & -1.2395 \\
\hline
\end{tabular}




$\begin{array}{lrrr}\text { H } & -2.665700 & 4.600900 & -0.422700 \\ \mathrm{H} & -2.294400 & 4.694400 & -2.178600 \\ \mathrm{H} & -2.412600 & 3.095600 & -1.363200 \\ \mathrm{C} & 0.226600 & 6.054100 & -0.675000 \\ \mathrm{H} & 1.318600 & 6.195800 & -0.530900 \\ \mathrm{H} & -0.072100 & 6.613400 & -1.590300 \\ \mathrm{H} & -0.291300 & 6.516400 & 0.191900 \\ \mathrm{C} & 0.348200 & 3.152700 & 0.623800 \\ \mathrm{C} & 1.775100 & 3.604200 & 0.905400 \\ \mathrm{H} & 2.012000 & 4.587500 & 1.358300 \\ \mathrm{C} & 2.811600 & 2.761200 & 0.604000 \\ \mathrm{H} & 3.853200 & 3.093000 & 0.801600 \\ \mathrm{C} & 3.789600 & 0.824700 & -0.466600 \\ \mathrm{C} & 4.523900 & 1.694400 & -1.541000 \\ \mathrm{H} & 4.816300 & 2.670000 & -1.095100 \\ \mathrm{H} & 3.800100 & 1.921300 & -2.354600 \\ \mathrm{C} & 4.821600 & 0.485100 & 0.659000 \\ \mathrm{H} & 4.312000 & -0.150100 & 1.414900 \\ \mathrm{H} & 5.128800 & 1.420600 & 1.176900 \\ \mathrm{C} & 3.398800 & -0.506600 & -1.151700 \\ \mathrm{H} & 2.875300 & -1.147500 & -0.412000 \\ \mathrm{H} & 2.651400 & -0.301800 & -1.948600 \\ \mathrm{C} & 5.772500 & 0.978600 & -2.103900 \\ \mathrm{H} & 6.273000 & 1.633400 & -2.856200 \\ \mathrm{C} & 6.758300 & 0.672700 & -0.951800 \\ \mathrm{H} & 7.673700 & 0.174800 & -1.350500 \\ \mathrm{H} & 7.089200 & 1.623100 & -0.473300 \\ \mathrm{C} & 6.067900 & -0.233700 & 0.093500 \\ \mathrm{H} & 6.781600 & -0.455900 & 0.921500 \\ \mathrm{C} & 5.631500 & -1.552000 & -0.582700 \\ \mathrm{H} & 6.520500 & -2.095400 & -0.982800 \\ \mathrm{H} & 5.149300 & -2.219800 & 0.163900 \\ \mathrm{C} & 4.638200 & -1.235200 & -1.723200 \\ \mathrm{H} & 4.310800 & -2.185600 & -2.200300 \\ \mathrm{C} & 5.339000 & -0.344100 & -2.772700 \\ \mathrm{H} & 6.223900 & -0.871600 & -3.202100 \\ \mathrm{H} & 4.645300 & -0.132800 & -3.617000 \\ \mathrm{~N} & -2.604400 & -1.502000 & 0.063200 \\ \mathrm{~N} & 2.604400 & 1.502000 & 0.063200 \\ \mathrm{O} & -0.000000 & 0.000000 & 1.435200 \\ \mathrm{Si} & 0.640200 & -3.285300 & 2.225000 \\ \mathrm{Si} & 0.193500 & -4.181500 & -0.855100 \\ \mathrm{Si} & -0.640300 & 3.285300 & 2.224900 \\ \mathrm{Si} & -0.193500 & 4.181500 & -0.855100\end{array}$

Table S30. Cartesian coordinates of 2_K-free_Al $\mathbf{A l}_{2} \mathrm{H}_{4}$

Zero-point correction=

Thermal correction to Energy=

Thermal correction to Enthalpy=

Thermal correction to Gibbs Free Energy=

Sum of electronic and zero-point Energies=

Sum of electronic and thermal Energies=

Sum of electronic and thermal Enthalpies=

Sum of electronic and thermal Free Energies=
0.998835 (Hartree/Particle)

1.064413

1.065357

0.902334

$-3803.332002$

$-3803.266424$

$-3803.265480$

$-3803.428503$
Al $\quad-1.654600$
0.331800
0.488200
Al $\quad 1.654600$
0.331800
$-0.488200$
C $\quad-1.623200$
4.672000
0.834600 


\begin{tabular}{|c|c|c|c|}
\hline $\mathrm{H}$ & -0.715100 & 4.182700 & 1.238700 \\
\hline $\mathrm{H}$ & -1.298200 & 5.528500 & 0.205700 \\
\hline $\mathrm{H}$ & -2.202400 & 5.083700 & 1.689200 \\
\hline C & -1.829000 & 3.072200 & -1.838300 \\
\hline $\mathrm{H}$ & -2.473200 & 2.391200 & -2.434000 \\
\hline $\mathrm{H}$ & -1.635300 & 3.987200 & -2.439100 \\
\hline $\mathrm{H}$ & -0.871400 & 2.542400 & -1.653900 \\
\hline C & -4.241400 & 4.492000 & -0.662900 \\
\hline $\mathrm{H}$ & -4.842500 & 4.788500 & 0.222500 \\
\hline $\mathrm{H}$ & -3.926000 & 5.422100 & -1.187000 \\
\hline $\mathrm{H}$ & -4.904100 & 3.925600 & -1.350300 \\
\hline C & -2.319400 & 2.560900 & 3.719400 \\
\hline $\mathrm{H}$ & -1.972100 & 3.590900 & 3.500800 \\
\hline $\mathrm{H}$ & -2.699800 & 2.538500 & 4.764400 \\
\hline $\mathrm{H}$ & -1.438100 & 1.887900 & 3.667100 \\
\hline C & -4.335900 & 0.304600 & 3.150800 \\
\hline $\mathrm{H}$ & -3.567600 & -0.256800 & 3.736000 \\
\hline $\mathrm{H}$ & -5.191000 & 0.443800 & 3.850000 \\
\hline $\mathrm{H}$ & -4.685200 & -0.324800 & 2.305600 \\
\hline C & -5.145100 & 3.205700 & 2.747700 \\
\hline $\mathrm{H}$ & -5.994500 & 2.949700 & 2.078300 \\
\hline $\mathrm{H}$ & -5.517700 & 3.171600 & 3.796100 \\
\hline $\mathrm{H}$ & -4.850200 & 4.254400 & 2.529800 \\
\hline C & -3.123800 & 1.846400 & 0.706700 \\
\hline C & -4.263500 & 1.247100 & -0.112100 \\
\hline $\mathrm{H}$ & -5.249100 & 1.742800 & -0.199300 \\
\hline C & -4.049400 & 0.095500 & -0.825300 \\
\hline $\mathrm{H}$ & -4.868400 & -0.315200 & -1.451000 \\
\hline C & -2.766400 & -1.854100 & -1.508600 \\
\hline C & -1.381200 & -2.502300 & -1.284400 \\
\hline $\mathrm{H}$ & -1.222000 & -2.636100 & -0.192700 \\
\hline $\mathrm{H}$ & -0.590900 & -1.784600 & -1.621700 \\
\hline C & -3.831500 & -2.879100 & -0.994400 \\
\hline $\mathrm{H}$ & -4.851200 & -2.456300 & -1.130200 \\
\hline $\mathrm{H}$ & -3.682300 & -3.017200 & 0.098900 \\
\hline C & -2.960700 & -1.681600 & -3.048300 \\
\hline $\mathrm{H}$ & -2.191200 & -0.970000 & -3.418900 \\
\hline $\mathrm{H}$ & -3.952700 & -1.218900 & -3.248900 \\
\hline C & -1.248300 & -3.853100 & -2.021900 \\
\hline $\mathrm{H}$ & -0.232500 & -4.266800 & -1.839200 \\
\hline C & -2.320400 & -4.833100 & -1.494200 \\
\hline $\mathrm{H}$ & -2.170700 & -5.014600 & -0.407000 \\
\hline $\mathrm{H}$ & -2.227900 & -5.819100 & -2.007100 \\
\hline C & -3.721900 & -4.230300 & -1.737300 \\
\hline $\mathrm{H}$ & -4.501400 & -4.930000 & -1.354000 \\
\hline C & -3.930200 & -4.006200 & -3.253000 \\
\hline $\mathrm{H}$ & -3.875400 & -4.979000 & -3.796100 \\
\hline $\mathrm{H}$ & -4.946500 & -3.588900 & -3.440400 \\
\hline C & -2.850400 & -3.036300 & -3.786800 \\
\hline $\mathrm{H}$ & -3.000000 & -2.877100 & -4.880000 \\
\hline C & -1.450300 & -3.640100 & -3.538700 \\
\hline $\mathrm{H}$ & -1.345100 & -4.609700 & -4.079300 \\
\hline $\mathrm{H}$ & -0.663100 & -2.962400 & -3.937200 \\
\hline C & 1.829000 & 3.072200 & 1.838300 \\
\hline $\mathrm{H}$ & 2.473200 & 2.391200 & 2.433900 \\
\hline $\mathrm{H}$ & 1.635300 & 3.987300 & 2.439100 \\
\hline $\mathrm{H}$ & 0.871300 & 2.542400 & 1.653900 \\
\hline C & 1.623200 & 4.672000 & -0.834700 \\
\hline $\mathrm{H}$ & 0.715100 & 4.182700 & -1.238800 \\
\hline $\mathrm{H}$ & 1.298100 & 5.528500 & -0.205700 \\
\hline $\mathrm{H}$ & 2.202300 & 5.083700 & -1.689200 \\
\hline C & 4.241300 & 4.492000 & 0.662900 \\
\hline $\mathrm{H}$ & 4.842500 & 4.788500 & -0.222500 \\
\hline $\mathrm{H}$ & 3.926000 & 5.422100 & 1.187000 \\
\hline $\mathrm{H}$ & 4.904100 & 3.925700 & 1.350300 \\
\hline C & 4.336000 & 0.304600 & -3.150700 \\
\hline $\mathrm{H}$ & 3.567600 & -0.256800 & -3.736000 \\
\hline $\mathrm{H}$ & 5.191000 & 0.443800 & -3.850000 \\
\hline
\end{tabular}




\begin{tabular}{|c|c|c|c|}
\hline $\mathrm{H}$ & 4.685200 & -0.324800 & -2.305600 \\
\hline C & 2.319500 & 2.560900 & -3.719400 \\
\hline $\mathrm{H}$ & 1.972100 & 3.591000 & -3.500800 \\
\hline $\mathrm{H}$ & 2.699900 & 2.538400 & -4.764400 \\
\hline $\mathrm{H}$ & 1.438000 & 1.887900 & -3.667100 \\
\hline C & 5.145100 & 3.205700 & -2.747700 \\
\hline $\mathrm{H}$ & 5.994500 & 2.949700 & -2.078300 \\
\hline $\mathrm{H}$ & 5.517700 & 3.171600 & -3.796100 \\
\hline $\mathrm{H}$ & 4.850200 & 4.254400 & -2.529800 \\
\hline C & 3.123800 & 1.846400 & -0.706600 \\
\hline C & 4.263400 & 1.247100 & 0.112100 \\
\hline $\mathrm{H}$ & 5.249100 & 1.742800 & 0.199300 \\
\hline C & 4.049400 & 0.095500 & 0.825300 \\
\hline $\mathrm{H}$ & 4.868400 & -0.315200 & 1.451000 \\
\hline C & 2.766400 & -1.854100 & 1.508600 \\
\hline C & 3.831500 & -2.879000 & 0.994400 \\
\hline $\mathrm{H}$ & 4.851200 & -2.456300 & 1.130200 \\
\hline $\mathrm{H}$ & 3.682300 & -3.017100 & -0.098900 \\
\hline C & 2.960700 & -1.681600 & 3.048300 \\
\hline $\mathrm{H}$ & 2.191200 & -0.970000 & 3.418900 \\
\hline H & 3.952700 & -1.218800 & 3.248900 \\
\hline C & 1.381200 & -2.502300 & 1.284400 \\
\hline $\mathrm{H}$ & 0.590900 & -1.784700 & 1.621600 \\
\hline $\mathrm{H}$ & 1.222100 & -2.636200 & 0.192700 \\
\hline C & 3.721900 & -4.230300 & 1.737300 \\
\hline $\mathrm{H}$ & 4.501400 & -4.930000 & 1.354000 \\
\hline C & 3.930200 & -4.006200 & 3.253000 \\
\hline $\mathrm{H}$ & 3.875400 & -4.979000 & 3.796100 \\
\hline $\mathrm{H}$ & 4.946500 & -3.588800 & 3.440500 \\
\hline C & 2.850400 & -3.036300 & 3.786800 \\
\hline $\mathrm{H}$ & 3.000000 & -2.877100 & 4.880000 \\
\hline C & 1.450300 & -3.640100 & 3.538700 \\
\hline $\mathrm{H}$ & 1.345100 & -4.609800 & 4.079300 \\
\hline $\mathrm{H}$ & 0.663100 & -2.962400 & 3.937200 \\
\hline C & 1.248400 & -3.853100 & 2.021900 \\
\hline $\mathrm{H}$ & 0.232600 & -4.266800 & 1.839200 \\
\hline C & 2.320500 & -4.833100 & 1.494200 \\
\hline $\mathrm{H}$ & 2.227900 & -5.819100 & 2.007100 \\
\hline $\mathrm{H}$ & 2.170700 & -5.014600 & 0.406900 \\
\hline $\mathrm{N}$ & -2.839500 & -0.575200 & -0.797600 \\
\hline $\mathrm{N}$ & 2.839500 & -0.575200 & 0.797600 \\
\hline 0 & -0.000000 & 0.723800 & 0.000000 \\
\hline $\mathrm{Si}$ & -2.687000 & 3.457100 & -0.186200 \\
\hline $\mathrm{Si}$ & -3.672700 & 1.988800 & 2.501800 \\
\hline Si & 2.686900 & 3.457100 & 0.186200 \\
\hline $\mathrm{Si}$ & 3.672700 & 1.988800 & -2.501800 \\
\hline $\mathrm{Al}$ & 1.635200 & -1.228800 & -2.624600 \\
\hline $\mathrm{H}$ & 2.242500 & -2.771500 & -2.659600 \\
\hline $\mathrm{H}$ & 1.137400 & -0.964400 & -4.181000 \\
\hline $\mathrm{Al}$ & -1.635200 & -1.228800 & 2.624600 \\
\hline $\mathrm{H}$ & -1.137400 & -0.964400 & 4.181000 \\
\hline $\mathrm{H}$ & -2.242500 & -2.771500 & 2.659700 \\
\hline
\end{tabular}

Table S31. Cartesian coordinates of $\mathrm{Al}_{2} \mathrm{H}_{4}$

Zero-point correction=

Thermal correction to Energy=

0.024827 (Hartree/Particle)

Thermal correction to Enthalpy=

0.030128

0.031072

Thermal correction to Gibbs Free Energy=

$-0.000632$

Sum of electronic and zero-point Energies=

$-487.229192$

$-487.223891$

Sum of electronic and thermal Energies=

$-487.222947$

Sum of electronic and thermal Enthalpies=

$-487.254651$
Al $\quad 0.000000$
$-0.000000$
1.297600
H $\quad-0.964700$
0.965600
2.154300 


$\begin{array}{lrrr}\text { H } & 0.964700 & -0.965600 & 2.154300 \\ \text { Al } & 0.000000 & -0.000000 & -1.297600 \\ \text { H } & 0.964700 & 0.965600 & -2.154300 \\ \text { H } & -0.964700 & -0.965600 & -2.154300\end{array}$

Table S32. Cartesian coordinates of AIOAI_1

Zero-point correction=

Thermal correction to Energy=

Thermal correction to Enthalpy=

Thermal correction to Gibbs Free Energy=

Sum of electronic and zero-point Energies=

Sum of electronic and thermal Energies=

Sum of electronic and thermal Enthalpies=

Sum of electronic and thermal Free Energies=

\author{
0.136820 (Hartree/Particle) \\ 0.150985 \\ 0.151929 \\ 0.097000 \\ $-719.463726$ \\ $-719.449562$ \\ $-719.448618$ \\ $-719.503547$
}

$\begin{array}{lrrr}\text { Al } & 1.270700 & -0.000000 & -0.211400 \\ \text { Al } & -1.270700 & 0.000000 & -0.211400 \\ \text { C } & 2.577600 & 1.613800 & -0.097000 \\ \text { C } & -2.577600 & -1.613800 & -0.097200 \\ \text { O } & 0.000000 & -0.000100 & 1.076400 \\ \text { C } & 2.577700 & -1.613800 & -0.097200 \\ \text { C } & -2.577600 & 1.613800 & -0.097000 \\ \text { H } & 3.159700 & -1.611300 & 0.864300 \\ \text { H } & 2.045500 & -2.594600 & -0.141400 \\ \text { H } & 3.338800 & -1.627900 & -0.919400 \\ \text { H } & 2.045400 & 2.594700 & -0.141200 \\ \text { H } & 3.159500 & 1.611300 & 0.864500 \\ \text { H } & 3.338700 & 1.628000 & -0.919100 \\ \text { H } & -2.045400 & 2.594700 & -0.141100 \\ \text { H } & -3.338800 & 1.628000 & -0.919100 \\ \text { H } & -3.159500 & 1.611300 & 0.864600 \\ \text { H } & -3.338800 & -1.627900 & -0.919300 \\ \text { H } & -2.045500 & -2.594600 & -0.141400 \\ \text { H } & -3.159600 & -1.611300 & 0.864400\end{array}$

Table S33. Cartesian coordinates of AIOAI_1_Al $\mathbf{A}_{2} \mathrm{H}_{4}$

Zero-point correction=

Thermal correction to Energy=

Thermal correction to Enthalpy=

Thermal correction to Gibbs Free Energy=

Sum of electronic and zero-point Energies=

Sum of electronic and thermal Energies=

Sum of electronic and thermal Enthalpies=

Sum of electronic and thermal Free Energies=

\author{
0.162262 (Hartree/Particle) \\ 0.183840 \\ 0.184784 \\ 0.109867 \\ $-1206.831066$ \\ $-1206.809488$ \\ $-1206.808544$ \\ $-1206.883461$
}

$\begin{array}{lrrr}\text { Al } & -1.658100 & 0.589300 & 0.202200 \\ \text { Al } & 1.658100 & -0.589300 & 0.202200 \\ \text { C } & -2.383300 & 0.893700 & 2.082200 \\ \text { C } & 2.383300 & -0.893700 & 2.082200 \\ \text { O } & 0.000000 & 0.000000 & 0.164900 \\ \text { C } & -1.951400 & 2.272400 & -0.905100 \\ \text { C } & 1.951400 & -2.272400 & -0.905100 \\ \text { H } & -3.022600 & 2.588400 & -0.934100 \\ \text { H } & -1.371900 & 3.132800 & -0.489000 \\ \text { H } & -1.622300 & 2.148300 & -1.962600 \\ \text { H } & -2.296600 & -0.016200 & 2.720300 \\ \text { H } & -1.824300 & 1.707000 & 2.607000 \\ \text { H } & -3.461100 & 1.188400 & 2.083100 \\ \text { H } & 1.622300 & -2.148300 & -1.962700\end{array}$




$\begin{array}{lrrr}\mathrm{H} & 3.022600 & -2.588400 & -0.934100 \\ \mathrm{H} & 1.371800 & -3.132800 & -0.489000 \\ \mathrm{H} & 3.461100 & -1.188400 & 2.083100 \\ \mathrm{H} & 2.296600 & 0.016100 & 2.720400 \\ \mathrm{H} & 1.824300 & -1.707100 & 2.607000 \\ \mathrm{Al} & -3.187100 & -1.401400 & -0.899800 \\ \mathrm{H} & -3.835600 & -2.777900 & -0.181300 \\ \mathrm{H} & -3.617900 & -1.704700 & -2.497300 \\ \mathrm{Al} & 3.187100 & 1.401400 & -0.899800 \\ \mathrm{H} & 3.617900 & 1.704700 & -2.497300 \\ \mathrm{H} & 3.835600 & 2.777900 & -0.181300\end{array}$

Table S34. Cartesian coordinates of AIOAI_2

\section{Zero-point correction=}

Thermal correction to Energy=

Thermal correction to Enthalpy=

Thermal correction to Gibbs Free Energy=

Sum of electronic and zero-point Energies=

Sum of electronic and thermal Energies=

Sum of electronic and thermal Enthalpies=

Sum of electronic and thermal Free Energies=

\author{
0.323542 (Hartree/Particle) \\ 0.348045 \\ 0.348989 \\ 0.268539 \\ $-1248.844285$ \\ $-1248.819782$ \\ $-1248.818837$ \\ $-1248.899288$
}

$\begin{array}{lrrr}\text { Al } & 0.779600 & -0.000200 & -0.967500 \\ \text { Al } & -0.779600 & -0.000200 & 0.967500 \\ \text { O } & 0.000000 & 1.322900 & 0.000000 \\ \mathrm{C} & 0.747300 & -0.013500 & -2.966800 \\ \mathrm{C} & -0.747300 & -0.013500 & 2.966800 \\ \mathrm{C} & 4.752000 & -0.824700 & 0.398500 \\ \mathrm{C} & 4.595200 & 0.439400 & 0.913200 \\ \mathrm{H} & 5.567300 & -1.547000 & 0.510900 \\ \mathrm{H} & 5.251700 & 1.034000 & 1.557200 \\ \mathrm{C} & -4.752000 & -0.824700 & -0.398500 \\ \mathrm{C} & -4.595200 & 0.439400 & -0.913200 \\ \mathrm{H} & -5.567300 & -1.547000 & -0.510900 \\ \mathrm{H} & -5.251700 & 1.034000 & -1.557200 \\ \mathrm{C} & 2.735100 & -0.031300 & -0.316200 \\ \mathrm{C} & -2.735100 & -0.031300 & 0.316200 \\ \mathrm{~N} & 3.367700 & 0.908700 & 0.462600 \\ \mathrm{~N} & 3.613200 & -1.090700 & -0.352000 \\ \mathrm{~N} & -3.613200 & -1.090700 & 0.352000 \\ \mathrm{~N} & -3.367700 & 0.908700 & -0.462600 \\ \mathrm{C} & 2.818100 & 2.236200 & 0.738600 \\ \mathrm{H} & 3.279100 & 2.991600 & 0.068000 \\ \mathrm{H} & 1.713500 & 2.190700 & 0.565000 \\ \mathrm{H} & 3.028200 & 2.510700 & 1.791400 \\ \mathrm{C} & 3.323000 & -2.336900 & -1.046300 \\ \mathrm{H} & 2.245900 & -2.579500 & -0.899100 \\ \mathrm{H} & 3.524300 & -2.251000 & -2.133700 \\ \mathrm{H} & 3.942100 & -3.151500 & -0.624200 \\ \mathrm{C} & -3.323000 & -2.336900 & 1.046300 \\ \mathrm{H} & -2.245900 & -2.579500 & 0.899000 \\ \mathrm{H} & -3.524300 & -2.251000 & 2.133600 \\ \mathrm{H} & -3.942100 & -3.151500 & 0.624100 \\ \mathrm{C} & -2.818100 & 2.236200 & -0.738500 \\ \mathrm{H} & -3.279100 & 2.991600 & -0.068000 \\ \mathrm{H} & -1.713500 & 2.190700 & -0.564900 \\ \mathrm{H} & -3.028200 & 2.510700 & -1.791300 \\ \mathrm{H} & 0.293000 & -0.080100 & 3.352900 \\ \mathrm{H} & -1.191900 & 0.918600 & 3.384400 \\ \mathrm{H} & -1.307800 & -0.866900 & 3.409800 \\ \mathrm{H} & 1.307800 & -0.866800 & -3.409800 \\ \mathrm{H} & -0.293000 & -0.080100 & -3.352900 \\ \mathrm{H} & 1.191900 & 0.918700 & -3.384400\end{array}$


Table S35. Cartesian coordinates of AIOAI_2_Al $\mathrm{H}_{4}$

Zero-point correction=

Thermal correction to Energy=

Thermal correction to Enthalpy=

Thermal correction to Gibbs Free Energy=

Sum of electronic and zero-point Energies=

Sum of electronic and thermal Energies=

Sum of electronic and thermal Enthalpies=

Sum of electronic and thermal Free Energies=
0.349741 (Hartree/Particle)

0.381664

0.382608

0.281607

$-1736.127316$

$-1736.095393$

$-1736.094449$

$-1736.195450$

\begin{tabular}{|c|c|c|c|}
\hline$A l$ & -1.631200 & 1.082700 & -0.234000 \\
\hline $\mathrm{Al}$ & 1.631100 & 1.082800 & 0.234000 \\
\hline 0 & 0.000000 & 0.416900 & -0.000200 \\
\hline$A l$ & -2.388100 & 2.399900 & 1.930000 \\
\hline $\mathrm{H}$ & -2.384900 & 4.007500 & 2.188800 \\
\hline $\mathrm{H}$ & -2.882900 & 1.640700 & 3.295000 \\
\hline$A l$ & 2.388600 & 2.400200 & -1.929600 \\
\hline $\mathrm{H}$ & 2.883700 & 1.641000 & -3.294500 \\
\hline $\mathrm{H}$ & 2.385600 & 4.007800 & -2.188300 \\
\hline C & -3.712400 & -2.523900 & 0.865500 \\
\hline $\mathrm{C}$ & -4.611500 & -2.081100 & -0.072700 \\
\hline $\mathrm{H}$ & -3.740800 & -3.394800 & 1.528700 \\
\hline $\mathrm{H}$ & -5.575400 & -2.491200 & -0.391800 \\
\hline $\mathrm{C}$ & 3.712500 & -2.523700 & -0.865800 \\
\hline C & 4.611300 & -2.081200 & 0.072800 \\
\hline $\mathrm{H}$ & 3.741000 & -3.394500 & -1.529200 \\
\hline $\mathrm{H}$ & 5.575100 & -2.491500 & 0.392100 \\
\hline C & -1.901200 & 1.972100 & -2.014300 \\
\hline C & 1.900700 & 1.971900 & 2.014500 \\
\hline$C$ & -2.854000 & -0.627000 & -0.060100 \\
\hline $\mathrm{C}$ & 2.854000 & -0.626900 & 0.060000 \\
\hline $\mathrm{N}$ & -4.067100 & -0.927900 & -0.626200 \\
\hline $\mathrm{N}$ & -2.651700 & -1.629400 & 0.853600 \\
\hline $\mathrm{N}$ & 2.651800 & -1.629100 & -0.854000 \\
\hline $\mathrm{N}$ & 4.066900 & -0.928100 & 0.626400 \\
\hline $\mathrm{C}$ & -4.729500 & -0.127200 & -1.652400 \\
\hline $\mathrm{H}$ & -5.596600 & 0.416400 & -1.223700 \\
\hline $\mathrm{H}$ & -4.005800 & 0.605100 & -2.051900 \\
\hline $\mathrm{H}$ & -5.082300 & -0.780600 & -2.474700 \\
\hline$C$ & -1.497000 & -1.695600 & 1.752400 \\
\hline $\mathrm{H}$ & -0.702800 & -1.042300 & 1.326700 \\
\hline $\mathrm{H}$ & -1.779800 & -1.331500 & 2.761300 \\
\hline $\mathrm{H}$ & -1.141500 & -2.742500 & 1.822100 \\
\hline $\mathrm{C}$ & 4.729000 & -0.127700 & 1.652900 \\
\hline $\mathrm{H}$ & 5.596300 & 0.415800 & 1.224700 \\
\hline $\mathrm{H}$ & 4.005200 & 0.604600 & 2.052400 \\
\hline $\mathrm{H}$ & 5.081400 & -0.781300 & 2.475300 \\
\hline $\mathrm{C}$ & 1.497500 & -1.694900 & -1.753200 \\
\hline $\mathrm{H}$ & 0.703200 & -1.041800 & -1.327600 \\
\hline $\mathrm{H}$ & 1.780600 & -1.330500 & -2.761900 \\
\hline $\mathrm{H}$ & 1.141900 & -2.741800 & -1.823400 \\
\hline $\mathrm{H}$ & 2.875700 & 2.493700 & 2.144800 \\
\hline $\mathrm{H}$ & 1.130600 & 2.772700 & 2.107900 \\
\hline $\mathrm{H}$ & 1.756700 & 1.297900 & 2.889700 \\
\hline $\mathrm{H}$ & -1.757800 & 1.298200 & -2.889700 \\
\hline $\mathrm{H}$ & -2.876100 & 2.494200 & -2.144100 \\
\hline $\mathrm{H}$ & -1.130900 & 2.772700 & -2.107900 \\
\hline
\end{tabular}




\section{References}

[S1] Koshino, K.; Kinjo, R. Construction of $\sigma$-Aromatic AlB2 Ring via Borane Coupling with a Dicoordinate Cyclic (Alkyl)(Amino)Aluminyl Anion. J. Am. Chem. Soc. 2020, 142, 90579062.

[S2] Gaussian 09, Revision E.01, Frisch, M. J.; Trucks, G. W.; Schlegel, H. B.; Scuseria, G. E.; Robb, M. A.; Cheeseman, J. R.; Scalmani, G.; Barone, V.; Mennucci, B.; Petersson, G. A.; Nakatsuji, H.; Caricato, M.; Li, X.; Hratchian, H. P.; Izmaylov, A. F.; Bloino, J.; Zheng, G.; Sonnenberg, J. L.; Hada, M.; Ehara, M.; Toyota, K.; Fukuda, R.; Hasegawa, J.; Ishida, M.; Nakajima, T.; Honda, Y.; Kitao, O.; Nakai, H.; Vreven, T.; Montgomery, J. A., Jr.; Peralta, J. E.; Ogliaro, F.; Bearpark, M.; Heyd, J. J.; Brothers, E.; Kudin, K. N.; Staroverov, V. N.; Keith, T.; Kobayashi, R.; Normand, J.; Raghavachari, K.; Rendell, A.; Burant, J. C.; lyengar, S. S.; Tomasi, J.; Cossi, M.; Rega, N.; Millam, J. M.; Klene, M.; Knox, J. E.; Cross, J. B.; Bakken, V.; Adamo, C.; Jaramillo, J.; Gomperts, R.; Stratmann, R. E.; Yazyev, O.; Austin, A. J.; Cammi, R.; Pomelli, C.; Ochterski, J. W.; Martin, R. L.; Morokuma, K.; Zakrzewski, V. G.; Voth, G. A.; Salvador, P.; Dannenberg, J. J.; Dapprich, S.; Daniels, A. D.; Farkas, O.; Foresman, J. B.; Ortiz, J. V.; Cioslowski, J.; Fox, D. J. Gaussian, Inc., Wallingford CT, 2013.

[S3] NBO 7.0, Glendening, E. D.; Badenhoop, J. K.; Reed, A. E.; Carpenter, J. E.; Bohmann, J. A.; Morales, C. M.; Karafiloglou, P.; Landis, C. R.; Weinhold, F. Theoretical Chemistry Institute, University of Wisconsis, Madison, WI, 2018.

[S4] Gaussian 16, Revision A.03, Frisch, M. J.; Trucks, G. W.; Schlegel, H. B.; Scuseria, G. E.; Robb, M. A.; Cheeseman, J. R.; Scalmani, G.; Barone, V.; Petersson, G. A.; Nakatsuji, H.; Li, X.; Caricato, M.; Marenich, A. V.; Bloino, J.; Janesko, B. G.; Gomperts, R.; Mennucci, B.; Hratchian, H. P.; Ortiz, J. V.; Izmaylov, A. F.; Sonnenberg, J. L.; Williams-Young, D.; Ding, F.; Lipparini, F.; Egidi, F.; Goings, J.; Peng, B.; Petrone, A.; Henderson, T.; Ranasinghe, D.; Zakrzewski, V. G.; Gao, J.; Rega, N.; Zheng, G.; Liang, W.; Hada, H.; Ehara, M.; Toyota, K.; Fukuda, R.; Hasegawa, J.; Ishida, M.; Nakajima, T.; Honda, Y.; Kitao, O.; Nakai, H.; Vreven, T.; Throssell, K.; Montgomery, J. A., Jr.; Peralta, J. E.; Ogliaro, F.; Bearpark, M. J.; Heyd, J. J.; Brothers, E. N.; Kudin, K. N.; Staroverov, V. N.; Keith, T. A.; Kobayashi, R.; Normand, J.; Raghavachari, K.; Rendell, A. P.; Burant, J. C.; lyengar, S. S.; Tomasi, J.; Cossi, M.; Millam, J. M.; Klene, M.; Adamo, C.; Cammi, R.; Ochterski, J. W.; Martin, R. L.; Morokuma, K.; Farkas, O.; Foresman, J. B.; Fox, D. J. Gaussian, Inc., Wallingford CT, 2016.

[S5] a) Grimme, S.; Antony, J.; Ehrlich, S.; Krieg, H. A consistent and accurate ab initio parametrization of density functional dispersion correction (DFT-D) for the 94 elements $\mathrm{H}$ Pu. J. Chem. Phys. 2010, 132, 154104-154119; b) Grimme, S.; Ehrlich, S.; Goerigk, L. Effect of the damping function in dispersion corrected density functional theory. J. Comput. Chem. 2011, 32, 1456-1465.

[S6] Lu, T.; Chen, F. Multiwfn: A multifunctional wavefunctional analyzer. J. Comput. Chem. 2012, 33, 580-592. 